\title{
Assessing the Effectiveness of Design Contracts as Test \\ Oracles in the Detection of Faults in Concurrent Object-Oriented Software
}

\author{
By \\ Wladimir Araujo, M. Eng., P. Eng.
}

A thesis submitted to the Faculty of Graduate Studies and Research

In partial fulfillment of the requirements for the degree of

Doctor of Philosophy in Electrical and Computer Engineering

Ottawa-Carleton Institute of Electrical and Computer Engineering

Department of Systems and Computer Engineering

Carleton University

Ottawa, Ontario, Canada, K1S 5B6

May 2010

Copyright (C) 2010 by Wladimir Araujo 
Library and Archives

Canada

Published Heritage

Branch

395 Wellington Street

Ottawa ON K1A ON4

Canada
Bibliothèque et

Archives Canada

Direction du

Patrimoine de l'édition

395 , rue Wellington

Ottawa ON K1A ON4

Canada
Your file Votre référence

ISBN: 978-0-494-70542-1

Our file Notre référence

ISBN: 978-0-494-70542-1
NOTICE:

The author has granted a nonexclusive license allowing Library and Archives Canada to reproduce, publish, archive, preserve, conserve, communicate to the public by telecommunication or on the Internet, loan, distribute and sell theses worldwide, for commercial or noncommercial purposes, in microform, paper, electronic and/or any other formats.

The author retains copyright ownership and moral rights in this thesis. Neither the thesis nor substantial extracts from it may be printed or otherwise reproduced without the author's permission.
AVIS:

L'auteur a accordé une licence non exclusive permettant à la Bibliothèque et Archives Canada de reproduire, publier, archiver, sauvegarder, conserver, transmettre au public par télécommunication ou par l'Internet, prêter, distribuer et vendre des thèses partout dans le monde, à des fins commerciales ou autres, sur support microforme, papier, électronique et/ou autres formats.

L'auteur conserve la propriété du droit d'auteur et des droits moraux qui protège cette thèse. $\mathrm{Ni}$ la thèse ni des extraits substantiels de celle-ci ne doivent être imprimés ou autrement reproduits sans son autorisation.
In compliance with the Canadian Privacy Act some supporting forms may have been removed from this thesis.

While these forms may be included in the document page count, their removal does not represent any loss of content from the thesis.
Conformément à la loi canadienne sur la protection de la vie privée, quelques formulaires secondaires ont été enlevés de cette thèse.

Bien que ces formulaires aient inclus dans la pagination, il n'y aura aucun contenu manquant. 


\begin{abstract}
Design by Contract (DbC) is a software development methodology that focuses on clearly defining the interfaces between components to produce better quality objectoriented software. The idea behind $\mathrm{DbC}$ is that a method defines a contract stating the requirements a client needs to fulfill to use it, the precondition, and the properties it ensures after its execution, the postcondition.
\end{abstract}

Though there exists ample support for $\mathrm{DbC}$ for sequential programs, applying $\mathrm{DbC}$ to concurrent programs presents several challenges. The first challenge is interference, the product of multiple threads of execution modifying and accessing shared data. The second is the specification of thread-safety properties (which objects are accessed by a single thread) in the presence of inheritance. The third is the specification of locking properties, which include the locks required to be held prior to a method execution and the order locks need to be acquired.

This work presents a solution to the problem of applying $\mathrm{DbC}$ to concurrent programs. Java is used as the target programming language and the Java Modelling Language (JML) as the specification language. This work defines what the above challenges are, propose changes and additions to JML and show how these constructs address such challenges. The new constructs are incorporated into the JML compiler to allow for the generation of Runtime Assertion Checking (RAC) code for such constructs. The compiler and its associated runtime libraries are modified to allow for the evaluation of assertion checking code in a concurrent environment.

Four case studies present a detailed evaluation of the applicability of DbC and the instrumentation techniques developed to concurrent systems. They use a highly concurrent industrial system. The first one assess the ability of the constructs introduced in JML to specify this product. The second one applies the modified JML compiler to this system and determines that the instrumented version can be used during system testing in place of the production version. This is done by analyzing the static and dynamic resource requirements introduced by the instrumentation overhead together with an 
analysis of the possible thread interleavings present in both versions. The third one studies the ability of the RAC code in detecting and diagnosing concurrent faults during system testing. Finally, the fourth assesses the effectiveness of contracts as test oracles by analyzing the observability and diagnosability of the instrumented version of the system with respect to functional faults. Based on these results, clear and objective requirements are defined for contracts to be effective test oracles for concurrent programs whilst balancing the effort in their design. 


\section{Acknowledgements}

This research was supported by Juniper Networks. I am extremely grateful for the support received not only through equipment time and funding but also the permission to publicize the results.

To Lionel and Yvan, I offer you my sincere appreciation for your time, your flexibility in adapting to my erratic schedule and your patience in reviewing this work many times. More importantly, however, I would like to thank you for teaching me the subtleties of good academic writing. I am still learning

Thanks to the JML community for the many fruitful discussions and the great software you produced. Special thanks to Gary Leavens and Clément Hurlin for the extensive discussions about JML and concurrency, which helped clarify fundamental points in this work.

Thanks simply do not do justice to the understanding of my friends during these years without Sundays and many other activities. I am happy to report that I am done.

The final thanks goes to my wife Scheila: although the contracts we work on are very different in nature, I am grateful for your appreciation to mine and your faith that this contract in particular was going to be fulfilled in its prescribed time. 


\section{Table of Contents}

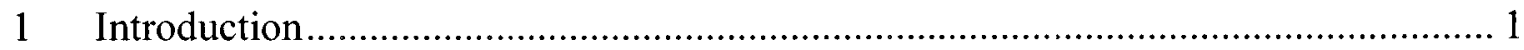

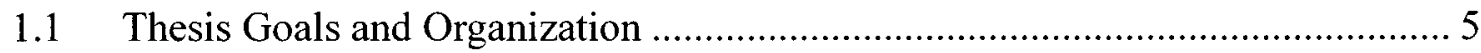

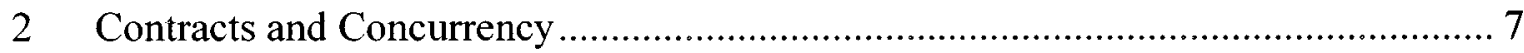

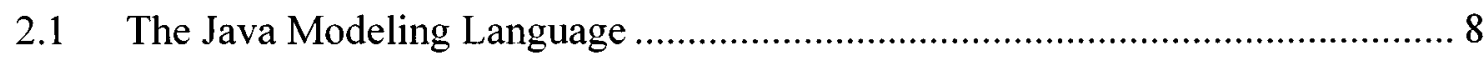

2.2 The Problem of Internal Interference ................................................................ 11

2.3 The Problem of External Interference ............................................................. 13

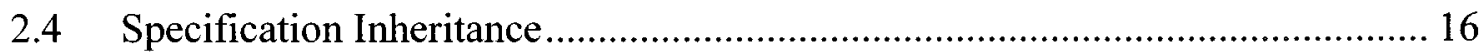

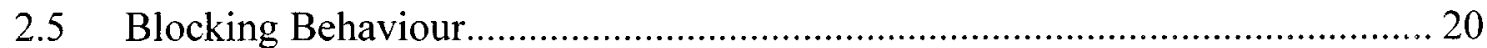

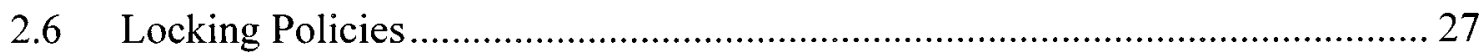

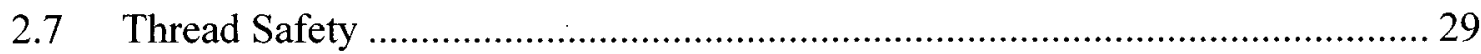

3 Specifying Contracts in the Presence of Concurrency ...............................................36

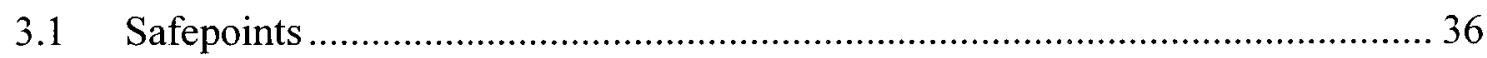

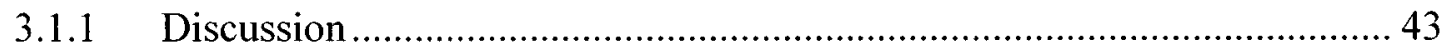

3.2 Specification of Thread-Safe Behaviour ............................................................ 45

3.3 Thread Safety + Safepoints $=$ No Interference.............................................. 53

3.3.1 Informal Semantics of Concurrent Contracts ........................................... 57

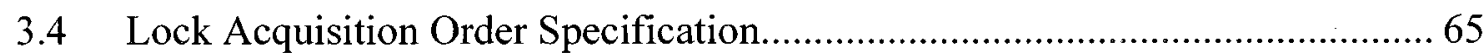

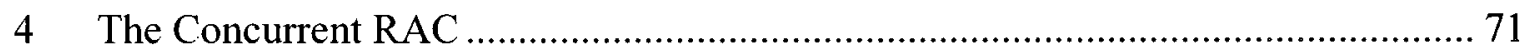

4.1 Runtime Assertion Checking Code Generation ............................................... 71

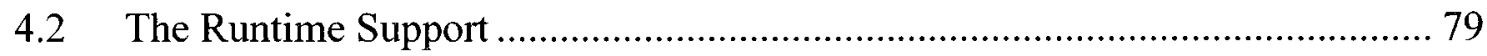

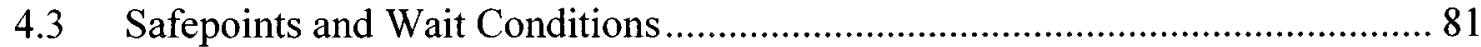

4.4 Thread-Safety Properties.............................................................................. 86

4.5 Verifying Lock Ordering Clauses ………........................................................ 93

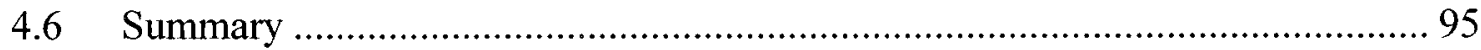

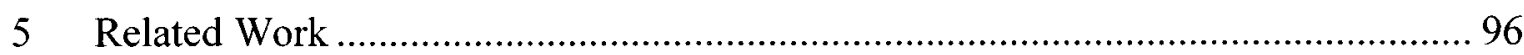

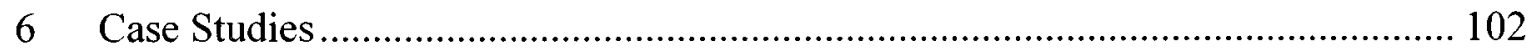

6.1 Target System and Test Bed Setup …………........................................... 102

6.2 Specification of an Industrial Concurrent System........................................... 104

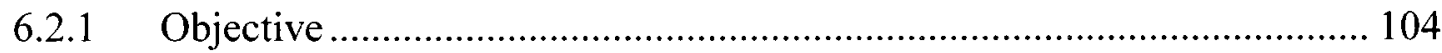




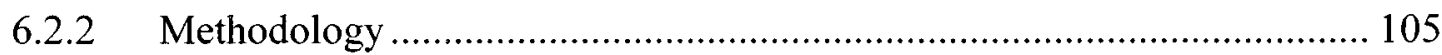

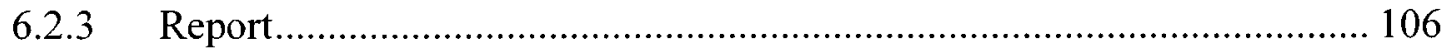

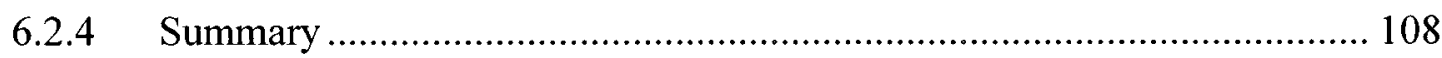

6.3 Applicability of Runtime Assertion Checking in the Verification of Concurrent

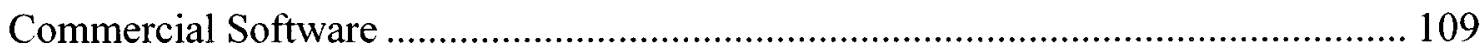

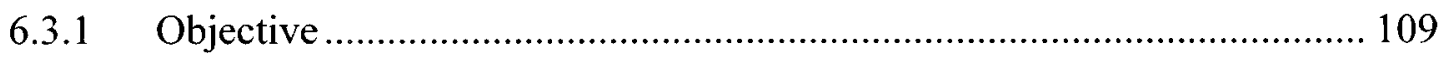

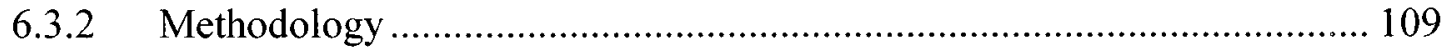

6.3.3 Report 1: Runtime Overhead ............................................................... 111

6.3.4 Report 2: Indistinguishability ………............................................... 118

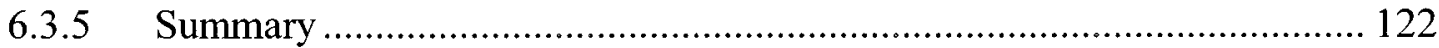

6.4 Observability and Diagnosability of Concurrency Related Faults in Industrial

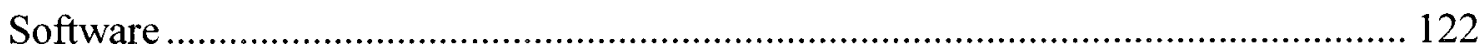

6.4.1 Background on Observability and Diagnosability .................................. 122

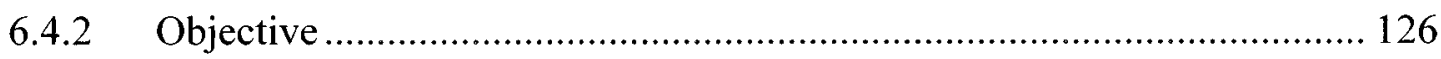

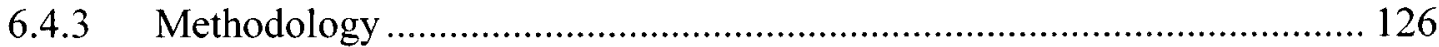

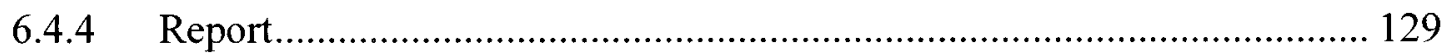

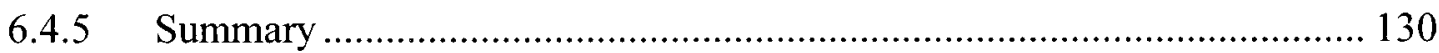

6.5 Observability and Diagnosability of Faults as a Function of Contract Complexity in Concurrent Industrial Software.......................................................... 131

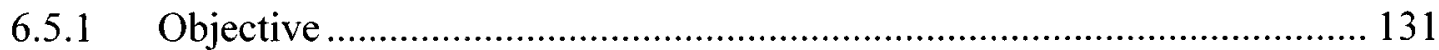

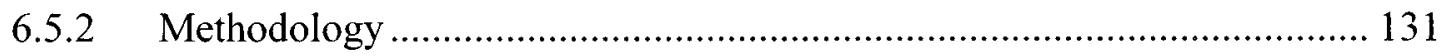

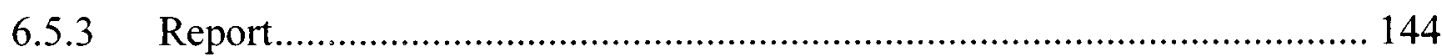

6.5.4 Discussion and Summary ................................................................... 162

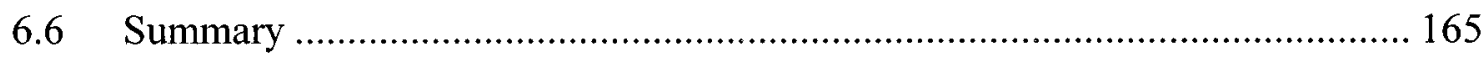

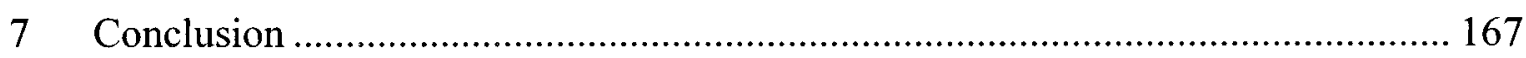

7.1 Summary of Contributions ............................................................................ 167

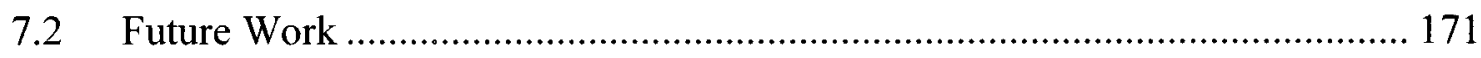

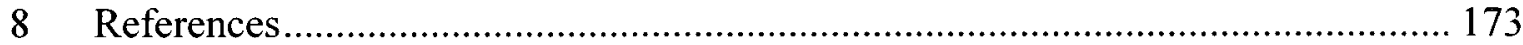

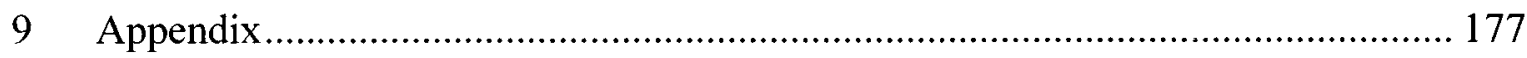

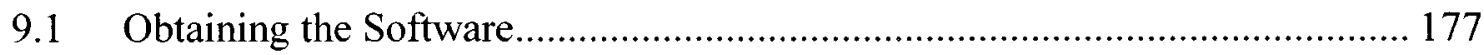

9.2 Using the Software ………........................................................................ 177 
9.3 Contract and Method Complexity Distributions for the Target System of the

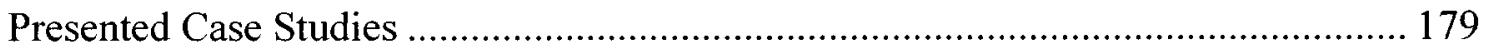




\section{List of Figures}

Figure 1: Sequence diagram illustrating the problem of internal interference. ............. 13

Figure 2: Sequence diagram depicting the problem of external interference ................ 14

Figure 3: Object diagram showing the two states of the tree. The mutated state depicts a rotation of the nodes from the initial state.

Figure 4: The modified specification production including the new concurrent-spec production 47

Figure 5: The productions specifying the newly introduced clauses.......................... 49

Figure 6: The productions specifying the lock order clause. 66

Figure 7: Object interconnection diagram to exemplify the semantics of the lock-orderclause.

Figure 8: Sequence diagram depicting an execution instance of method $a 1 \cdot m 1()$ for the running example. The notes attached to activations denote points in the execution referenced in the text and the lock order clauses in effect for such activation.

Figure 9: UML class diagram depicting the runtime support for concurrent checking and its relationship to an instrumented class $X$. The members of $X$ in this diagram as well as class $\mathrm{X}$ : : JMLLocalstate are generated during compilation. Class JMLChecker and its components are part of the JML runtime library. ThreadLocal and ConcurrentHashMap are part of the Java runtime library.

Figure 10: Test bed for case studies. Rectangles represent processes, the wide bidirectional arrows represent inter-process communication protocols, and the curved rectangles represent files locally produced or read by the associated processes.

Figure 11: Graph comparing the incremental class file size for classes instrumented using the technique for multi-threaded and sequential programs as a function of the number of methods in the non-instrumented class. The solid lines represent linear regressions for their respective series. 113

Figure 12: Comparison of the heap utilization between the production and the instrumented versions of the target system subject to the same test suite. 116 
Figure 13: Comparison of the CPU utilization between the production and the instrumented versions of the target system subject to the same test suite.

Figure 14: Diagnosability measure example: diagnosis flow as a sequence diagram.... 124

Figure 15: Graph of detected faults per contract type. The different series display the contributions of faults that required or not a contract to be updated to detect it as well as a cumulative detection rate with the increase of the contract complexity.. 145

Figure 16: Graph relating the CCM of contracts to the VG of the methods they specify. Each point in the graph is the maximum value of the CCM of a contract of a particular type that detected a fault.

Figure 17: Histogram of faults per detecting contracts' CCM.

Figure 18: The fault density distribution per method cyclomatic complexity. The method frequency for $\mathrm{VG}=4$ is $84.64 \%$. 152

Figure 19: Cumulative distribution of faults per detecting contracts' CCM for different types of contracts. $100 \%$ represents the total faults detected by the given type of contract. 153

Figure 20: Histogram and density of undetected faults per method cyclomatic complexity. 154

Figure 21: Histogram depicting the diagnostic effort according to the diagnosis scope measure for contracts of different types 156

Figure 22: Histogram depicting the diagnostic effort according to the distance measure for contracts of different types. The labels shown are for the bucket 5 . 157

Figure 23: Histogram depicting the diagnostic effort according to the weighed distance measure for contracts of different types. 158

Figure 24: Global diagnosability as a function of global observability. 162

Figure A.1: CLI options in EBNF format for the jml rac utility to perform thread-safety instrumentation of pre-compiled classes. 178

Figure A.2: Histogram of faults detected per method cyclomatic complexity. 179

Figure A.3: Histogram of methods per cyclomatic complexity in the system under test. The frequency axis is in logarithmic scale. 180 


\section{List of Code Excerpts}

Listing 1: JML specification for the method extract () of the LinkedQueue class.11

Listing 2: JML specification of method isEmpty () of class LinkedQueue. 15

Listing 3: JML specification of interface Channel. Only the receive method and relevant model fields are shown for simplicity......

Listing 4: JML specification of class PipedChannel. Only relevant methods, fields and specifications are shown for simplicity 18

Listing 5: The class Pipe and its JML specification. 22

Listing 6: JML specification of class BlockingChannel2. Irrelevant methods and fields are omitted for clarity.

Listing 7: Example of desugaring of method specifications. The two method specifications are equivalent. P, P1, Q, and Q1 represent JML predicates............. 25

Listing 8: The extended contract for the get () method of the class Pipe. 34

Listing 9: JML specification for the method extract() of the LinkedQueue class using safepoints to avoid internal interference.

Listing 10: Using safepoints to prevent external interference in the presence of blocking behaviour. Excerpt of Listing 5 showing only the modified method.

Listing 11: Method receive () of class PipedChannel equipped with safepoints. 40 Listing 12: Method receive() of class BlockingChannel2 equipped with safepoints. 43

Listing 13: Method declaration exemplifying the use of thread-safety specification clauses.

Listing 14: The contract for the get () method of the class Pipe using the new locking constructs.

Listing 15: Example of contract involving thread-safety requirements of method parameters. Q and S represent valid JML predicates. 54

Listing 16: Sample state machine designed for a concurrent environment. State changes are protected by synchronized methods. 56

Listing 17: Code fragments exemplifying the use of the lock-order-clause. 68 
Listing 18: Simplified wrapper method for method extract() of class LinkedQueue. 74

Listing 19: Simplified precondition assertion method for method extract () of class LinkedQueue. 76

Listing 20: Simplified postcondition assertion method for method extract () of class LinkedQueue. 76

Listing 21: Interface Channel (excerpt)................................................................... 77

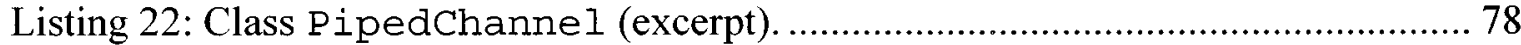

Listing 23: Access method to model field connected in class PipedChannel....... 78

Listing 24: Method extract () of class LinkedQueue using safepoints to avoid internal interference.

Listing 25: Simplified versions of precondition and postcondition safepoints methods. . 83 Listing 26: Simplified concurrent wrapper method for method extract () of class LinkedQueue. 85

Listing 27: Example of the use of instances of Lock. 87

Listing 28: Simplified concurrent assertion and evaluation methods for method extract () of class LinkedQueue. 89

Listing 29: Instrumented version of method extract () of class LinkedQueue...... 91

Listing 30: Simplified locking order assertion method for class LinkedQueue........... 94

Listing 31: Interface Central to demonstrate the different types of contracts............ 134

Listing 32: Contract for Central. open (), an example of a contract of type basic. 135 Listing 33: Contract for Central.open(), an example of a contract of type elementary. 135

Listing 34: Contract for Central. open(), an example of a contract of type advanced. 


\section{List of Tables}

Table 1: Statistics on the usage of the proposed thread-safety constructs for the case study.

Table 2: Statistics regarding the use of concurrency clauses not related to thread-safety

Table 3: Summary of concurrency related faults observed by contracts. Faults are classified according to their nature and if contracts were updated to enable detection.

Table 4: JML features allowed in contracts as a function of their type. 133

Table 5: Mapping from a JML contract (top, in EBNF format) to a Java program (bottom) used to measure a contract's complexity. Mapping for universal and existential quantifiers is also shown.

Table 6: Example of the calculation of the CCM of Central. open(), which refers to specification-only pure class Connection (not shown)

Table 7: Faults detected based on contract type and grouped by the need to update a contract to detect it. The rightmost column shows the cumulative totals the faults of a particular contract type. 144

Table 8: Summary of reasons for failing to detect faults through contracts. Frequencies are relative to undetectable faults. 148

Table 9: Statistics for the diagnostic effort according to the diagnosis scope measure for contracts of different types. 156

Table 10: Statistics for the diagnostic effort according to the distance measure for contracts of different types.

Table 11: Statistics for the diagnostic effort according to the weighed distance measure for contracts of different types. 158

Table 13: Percentage of faults located in the same method as the detecting contract. The first column considers only the detected faults for a particular contract type whilst the second considers all the faults, including the undetected ones. 


\section{List of Acronyms}

$\begin{array}{ll}\text { CCM } & \text { Contract Complexity Measure } \\ \text { COPS } & \text { Common Open Policy Service } \\ \text { COPS-PR } & \text { COPS for Policy Provisioning } \\ \text { DbC } & \text { Design by Contract } \\ \text { IETF } & \text { Internet Engineering Task Force } \\ \text { JML } & \text { Java Modelling Language } \\ \text { RFC } & \text { Request for Comment } \\ \text { RAC } & \text { Runtime Assertion Checking } \\ \text { SAE } & \text { Service Activation Engine } \\ \text { SRC } & \text { Session Resource Controller }\end{array}$




\section{INTRODUCTION}

Including specifications of program behaviour together with the source code is not a new idea. Design-by-Contract $(\mathrm{DbC})[1]$ is one of the more elaborate software development methodologies that put such idea in practice, with Eiffel being a well-known example of a programming language that supports it. Following DbC principles, a method defines a contract stating the requirements a client needs to fulfill to use it, the precondition, and the properties it ensures after its execution, the postcondition. Contracts can be treated as logical assertions about the state of a program at a certain point. A program can be instrumented with code that checks the validity of the assertions at runtime and upon failure throws an exception indicating where it happened. $\mathrm{DbC}$ also defines object invariants [2] $]^{1}$, properties that must hold in all visible states of an object. The visible states of an object are the states just after object construction, just before a visible method execution, and just after a visible method execution. Behavioural subtyping [3-7] is an integral part of DbC. A subtype automatically inherits the specification (contracts and invariants) from its super-types [8-10]. The effective precondition of a method is the disjunction of all the inherited preconditions and the method's declared preconditions. The effective postcondition is the conjunction of all inherited postconditions for which the associated precondition is satisfied and the method's declared postconditions if associated preconditions are satisfied. The effective class invariant is the conjunction of all inherited class invariants with the object's declared invariant. This guarantees that a subtype can be properly used in place of its super-type(s).

The Java Programming Language [11] does not provide native support to DbC. It only provides basic support for assertions through the assert keyword, which simply causes an exception to be thrown in case a given Boolean expression evaluates to false. This work uses the Java Modeling Language (JML) $[12,13]$ as the specification language used

\footnotetext{
'Meyer originally named them class invariants but the author prefers the term object invariant since it is an invariant about an object.
} 
to write contracts. It allows the specification of properties from simple assertions (lightweight properties) about pointer null-ness to complete functional correctness of program components (strong properties). JML is a behavioural interface specification language with which one can specify the syntactic and behavioural interface of a portion of Java code ${ }^{2}$. It also includes notations for pre- and postconditions, invariants, and offers mechanisms for specification inheritance, thus providing support for the Design-byContract paradigm. In the context of JML, the only types of methods that are not considered visible are the ones marked as helpers. Such methods, which do not implicitly rely on the object invariant, are also not obliged to establish it. They are useful to establish intermediate states as factored out routines used by other, possibly visible, methods. JML has a Java-like syntax and specifications can even perform method calls in assertions. It also provides a rich set of model classes (i.e. classes that can only be used in specifications) that can be used to construct rich abstract descriptions of program behaviour, such as data structure model classes, which can be used to model abstract properties of concrete data structures in a concise way.

The JML toolset comes with a compiler that translates specifications into runtime assertion checking (RAC) code producing Java classes augmented with executable assertions. The process of adding RAC code to a Java class is called instrumentation. The resulting class is called the instrumented class. The JML compiler [14] produces RAC code that enforces behavioural subtyping, i.e. RAC code for all applicable invariants and preconditions is executed upon entering a method, and $\mathrm{RAC}$ code for all applicable postconditions and invariants is executed upon exiting a method.

Most work on DbC focused on sequential programs, and applying DbC to concurrent programs presents several challenges. The first challenge is interference, the product of multiple threads of execution modifying and accessing shared state. Interference is present even on correct programs with respect to concurrency control. Basically, interference with respect to the precondition happens because assertion checking code is evaluated at a point in time after which other threads are allowed to modify the objects

\footnotetext{
2 JML allows one to specify loop variants and invariants, internal method properties, external method properties and properties of types (classes and interfaces).
} 
referenced in such assertions but prior to the point in which these objects are accessed by the method in question. This causes RAC code to report errors for correct methods and vice-versa. The problem is analogous with respect to postconditions and invariants. Solving the issue of interference with a focus on runtime assertion checking is one major contribution of this work.

The second challenge is the specification and verification of locking related properties. It is common practice to have methods in a concurrent class (informally) specify which locks the client is required to hold prior to executing such method. It is also common practice to specify which locks the method in question is going to (potentially) acquire during its execution. In some cases, it is also useful to specify the order in which certain locks must be acquired to avoid deadlocks. The use of locking policies is a common deadlock avoidance technique [15]. JML already has constructs to deal with the first two cases (the construct addressing the first case presents inheritance issues, though). This work introduces the concept of a lock order clause, which is a specialized construct to specify only lock acquisition order expressions. Its semantics makes the use of quantifiers unnecessary and also eliminates the soundness issues with the current approach.

The third challenge is the specification of thread-safety properties in the presence of inheritance. These properties state which objects are safe to be accessed by the current executing thread, i.e. there are no other threads accessing such objects. To our knowledge, locking requirements have so far been associated with preconditions. This causes two problems. It implicitly associates locking requirements with behavioural subtyping: satisfying them becomes optional since inherited preconditions are disjoined with the local one, and sub-types with potentially different concurrency control strategies are forced to inherit possibly meaningless locking requirements. The same problem happens with thread-safety predicates. These properties only make sense in the context of the target type, not its hierarchy. This work solves this problem by detaching these properties from preconditions while considering interference issues. This is another contribution of this work. 
The JML compiler (jmlc) provides support for generating RAC code for sequential programs. The generated code depends on some infrastructure code to be available when executing the instrumented program. This code is called the runtime. This work modifies the runtime to support the execution of multi-threaded programs. These modifications relate to the bookkeeping necessary for the correct execution of recursive instrumented code and the locks each thread has acquired, including the acquisition order. The JML compiler is modified to generate RAC code for the introduced constructs, as well as other concurrency related constructs previously described in the literature [16] but never implemented in a RAC scenario.

The purpose of developing techniques to extend $\mathrm{DbC}$ to concurrent programs is to enable the automatic generation of test oracles from contracts. It is understood that the task of creating test oracles is time-consuming and represents a significant portion of the testing effort. It is also known [17] that such task is error-prone due to its inherent complexity and the absence of a general design methodology. Automatically deriving test oracles from contracts through the generation of executable assertions attempts to address these issues. This thesis presents four case studies that evaluate the applicability of contracts to concurrent programs as a method of deriving test oracles. Each case explores a different aspect of the same target system, a highly concurrent software product of the computer networks industry,

The first case study evaluates if the proposes constructs can be used to specify the behaviour presented by a complex concurrent program. The study covers both functional and concurrent properties. The second study evaluates the applicability of the instrumentation techniques by comparing the behaviour of the production system with its instrumented version. Both are required to be equivalent if one is to apply conclusions obtained through the execution of one version of the system to the other. It also evaluates if the overhead introduced by the instrumentation process allows a complex software system to be placed in the same test bed as its production version, a fundamental property if this technique is to be used in the industry. The third study focuses on the ability of the instrumented system in detecting and diagnosing race conditions and deadlocks. The fourth study analyzes the influence of contract complexity in its ability to detect and 
diagnose faults. It concludes with a recommendation on the contents of a contract to detect faults on a concurrent system considering the effort to design a contract and the incremental detection power this effort returns. These last two studies are performed by systematically injecting faults in the system and determining if the instrumented code can detect them as well as measuring the effort to diagnose them. This systematic approach combined with objective measures allows the production of clear and objective recommendations on the contents of contracts, likely the most significant contribution of this work.

By addressing the problem of creating contracts specifying concurrent properties as well as functional properties in a concurrent environment, implementing them in a compiler to generate instrumented code, verifying that an instrumented system can be used in place of its production version, and determining that such contracts can not only detect a significantly high number faults but to significantly reduce the effort in diagnosing them, this work provides a solution to the problem of applying Design by Contract to concurrent object-oriented software.

\subsection{Thesis Goals and Organization}

The following chapter describes the challenges to apply $\mathrm{DbC}$ to concurrent software in detail, presenting examples and identifying the issues in specifying various properties and behaviours related to concurrency. The next chapter presents the solutions to these problems. It describes the contributions of this work to JML to enable the specification of concurrent contracts, the first goal of this thesis. Chapter 4 describes the implementation of the proposed constructs in JML's RAC. It shows that the proposed constructs can be used to perform runtime assertion checking, the second goal of this thesis. Related work is discussed in the subsequent chapter. Chapter 6 describes a series of case studies based on an industrial system to demonstrate and evaluate several aspects of the techniques developed. The first two goals of the case studies are to carefully evaluate if the proposed constructs can be used to specify an industrial system and then to see if runtime assertion checking is feasible as a verification tool in an industrial setting. The third goal is to evaluate if contracts can be used as an effective and efficient mechanism to detect and 
diagnose concurrency related faults in industrial software. The fourth goal is to evaluate if contracts can be used as an effective and efficient mechanism to detect and diagnose functional faults in concurrent software. The fifth goal is to offer clear and objective guidelines on the contents of contracts balancing the effort in their design with the return in the detection and diagnosis of functional faults in concurrent systems. It concludes with a summary of its contributions and future work. 


\section{CONTRACTS AND CONCURRENCY}

This chapter presents the problems associated with using contracts to specify the behaviour of concurrent programs and to generate runtime assertion checking code from such contracts. Java and JML are used in all examples thorough this document. Although we do not describe JML in detail we present a brief description of the relevant constructs just enough for the understanding of the intent of a particular contract and the challenges in using it in a concurrent environment. We also generalize these problems to Java-like object-oriented languages and specification languages through the use of UML diagrams to describe the program under study and OCL to describe the contracts, respectively. We only require from the specification language that it be able to specify formulae for preconditions, postconditions and invariants, that it supports abstraction through model fields, and that it supports specification inheritance.

The problems presented in this chapter cover different aspects of the verification of concurrent contracts. Some of these problems have been originally presented in [16] or at least restated in the context of Java and JML. We find such solutions, although interesting, inadequate to the complete scope of an object-oriented language like Java, with intricate concurrency control mechanisms.

We start by discussing the problems of internal and external interference, originally presented in [16], or at least restated in the context of Java and JML. We defer the presentation of our solution to these problems until the next chapter since it is necessary to have a complete view of the problems it solves before evaluating its effectiveness.

We then move to the problem of specifying and verifying concurrent contracts in the presence of specification inheritance. Specification inheritance is the mechanism used by JML to achieve behavioural subtyping. This is an original contribution of this work.

We then continue to the problem of specifying and verifying blocking behaviour. A simple statement of the problem of specifying such behaviour was made in [16] as an adaptation of [18] to Java and JML. Such statement implies that the method being 
specified is atomic. We use the same constructs to specify blocking behaviour but we do not make any assumptions regarding atomicity. Furthermore, we present the problem taking inheritance into account, which has never been done before.

We tackle locking policies next. Although there are mechanisms to specify locking policies today [19], the current solution is not amenable to runtime assertion checking. We present such shortcomings. The solution is, again, deferred to the next chapter.

We finally address the problem of specifying and verifying thread-safe behaviour. The notion of thread-safety is borrowed from [16]. We restate the problem with a focus on runtime assertion checking (RAC) and show why the current approaches are insufficient. Furthermore, we bring specification inheritance into the picture and present several issues with combining thread-safety properties with functional properties. This is another contribution of our work.

\subsection{The Java Modeling Language}

The Java Modeling Language (JML) $[12,13]$ is a specification language used to write contracts. JML has a Java-like syntax and specifications can even perform method calls. JML specifications are delimited by the strings $/ * @$ and $\Theta * /$ or by the remainder of lines following $/ / \Theta$, being treated as comments by the Java compiler. Specifications can be written as annotations in '.java' files (javadoc style).

In JML, the interface of a method is specified through a set of clauses. The most relevant for this study are:

- requires: specifies the conditions that need to be satisfied by the method caller.

- ensures: specifies the properties that this method guarantees to its caller.

- when: specifies an enabling condition (the method blocks until this condition is met).

- signals: specifies a condition that is guaranteed to hold if a given exception is thrown. 
- signals_only: constrains the exceptions that can be thrown when a condition for exceptional behaviour is satisfied.

- normal_behaviour: specifies the conditions in which a method returns normally and what it ensures.

- exceptional_behaviour: specifies the conditions in which a method throws an exception.

Invariants are specified using the invariant clause. Invariants must hold in any publicly visible state, i.e. prior to and after the execution of any instance methods, with the exception of private methods marked with the helper modifier. These are methods used to establish intermediate states. History constraints (a type of invariant) specify how values can change between consecutive publicly visible states. The constraint clause is used to define history constraints.

JML provides a rich set of native operators for defining complex specifications, the most relevant for this study being:

- $\operatorname{lold}(e)$ : used in post-conditions to refer to the value of expression e in the prestate of the method. It is also used in history constraints.

- Ireturn: the return value of a method. Its type is the same as the method return type.

- \lockset: returns the set of locks held by the current thread.

- Operators $<$ and $<=$ : used to test the order of monitor lock acquisition. A lock is greater than another if it was acquired later.

- $\backslash \max (e)$ : returns the largest lock in a set according to the ordering defined by the operator above applied to a set of objects.

JML allows the use of model fields in specifications by using the modifier model in a declaration. These are fields accessible only to specification code, being ignored by Java 
code. They are treated as regular Java fields by specifications. Model fields are an important feature to cleanly support abstract specifications. Model fields can be present on interfaces on a per instance basis (by using the keyword instance), something Java does not allow. They are also inherited by classes implementing such interfaces or interfaces that extend them. This is also valid for model fields specified in classes. Model fields are realized by concrete classes through the represents clause, which maps model fields (directly or indirectly) to a set of Java fields.

A method is pure if it is declared with the modifier pure or is a member of a pure class or interface. Pure methods cannot diverge (i.e. they must terminate) and are not allowed side-effects (i.e. they cannot assign to locations that exist in the pre-state). Pure constructors cannot diverge and can only assign to the object being constructed. Only pure methods are allowed in assertions (e.g. in the requires and ensures clauses of a method specification).

Before discussing the example in Listing 1, we must recall the notions of a method's prestate and post-state [12]. "The pre-state of a method call is the state just after the method is called and parameters have been evaluated and passed, but before execution of the method's body. The post-state of a method call is the state just before the method returns or throws an exception; in JML we imagine that \result and information about exception results is recorded in the post-state" ([12], p. 8).

The method specification in Listing 1 (excerpt from [16]), composed of two specification cases separated by the keyword also (each with a precondition and the corresponding expected postcondition, the postcondition to be established if the precondition is satisfied), simply tells that the head of the list will move to the next element and the method will return the value of what used to be the first element of the list if the list is not empty (lines 5-9), and returns null otherwise (lines 1-4). In JML, the preconditions of a method (i.e., the requires clauses), as well as arguments to the lold operator in postconditions are evaluated in the method's pre-state. The method postconditions (i.e., the ensures clauses) are evaluated in the method's post-state. 
The type specification in Listing 1 contains a single object invariant head.value == nul.. The invariant predicate is evaluated in both the method's pre- and post-state.

\subsection{The Problem of Internal Interference}

Two threads interfere when one changes data the other observes. This becomes a problem if, due to an arbitrary interleaving, one thread's perception of the shared data is not true due to a modification made by another thread and it relies on such perception for future computations. The example below (from [16]) illustrates the situation quite well.

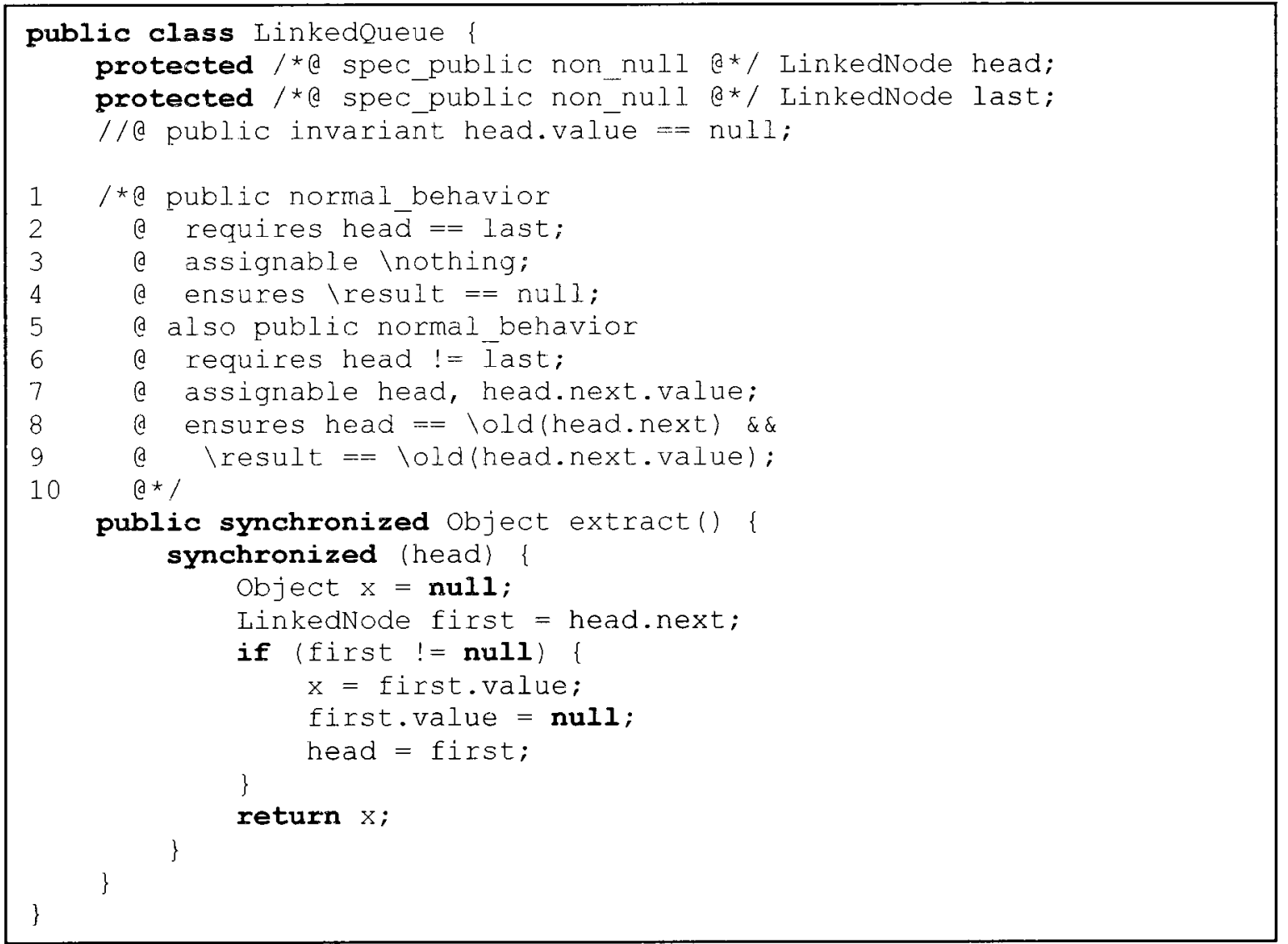

\section{Listing 1: JML specification for the method extract () of the Linkedqueue class.}

The preconditions of a method (i.e. the expression in the requires clause) are evaluated in the method's pre-state. Also, the expressions provided as arguments to the lold operator in the method postcondition are evaluated in the pre-state of the method. The method postconditions are evaluated in the method's post-state. 
Although clear and straightforward, this specification is not correct in a multi-threaded environment. Suppose that extract () is invoked by thread 1 and in the method's prestate, head references the same object as last (i.e. the list is empty). Suppose, also, that thread 2 pre-empts thread 1 right after it acquires the lock on this to fully execute the method insert(), which does not acquire such lock. The postcondition of such method is that head is not referencing the same object as last, i.e. the list is not empty. Once thread 1 resumes execution and acquires the lock on head, it will notice that there is an element on the list and will return it, violating the postcondition of this method for an empty list, i.e. that it should have returned null.

This example can also be visualized on the UML interaction diagram on Figure 1. We use the notation from the UML Profile for Schedulability, Performance and Time to depict concurrent execution by placing two activations side-by-side. In this example, it means that methods extract () and insert () of Linkedqueue are executed concurrently. The self message "eval precondition" flags the evaluation of the precondition and invariant predicates in the pre-state. At that point, threadl observes that head=null and thus selects the specification case in which \result=null is the expected postcondition. The method execution proper begins once this self-action returns. We label the points in which threadl is pre-empted and resumed using notes. Notice that extract () executes completely while thread1 is sleeping. Once thread1 resumes, it proceeds to return an element from the head of the queue (the one just inserted) therefore causing \result to not be null. The last step of the method execution is to execute the self-action "eval postcondition". At this point it recalls the selected postcondition in the pre-state and evaluates it. Although the behaviour of both methods is correct, the postcondition is not satisfied, producing a false negative. 


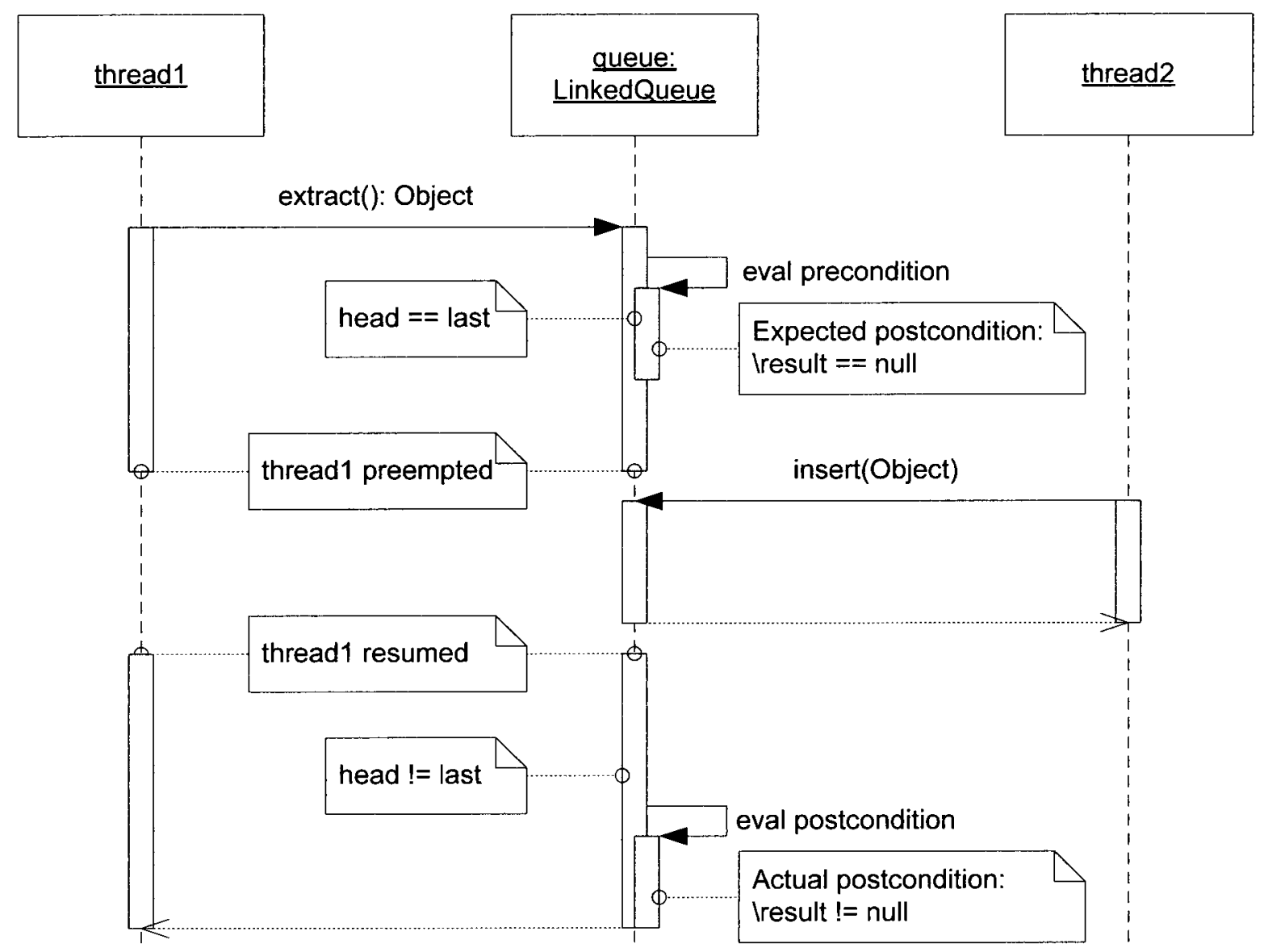

Figure 1: Sequence diagram illustrating the problem of internal interference.

The behaviour just described is called internal interference, when another thread affects the current thread's execution of a method by making changes observable by such method once it started executing [16]. This problem is not specific to Java or JML. Any object-oriented language in which the sequence diagram above is realizable and provides support for $\mathrm{DbC}$ via runtime assertion checking (RAC) is prone to the same issues. It is important to emphasize that such problem arises due to the combination of $\mathrm{DbC}$ and the program under execution. It is not due to erroneous concurrency control on the part of the implementation either of the client or the provider.

\subsection{The Problem of External Interference}

Another type of interference happens when a thread makes observable changes between a method call and a method's entry, or between a method's exit and the caller's resumption 
[16]. This is called external interference. Such behaviour can be seen in the following example.

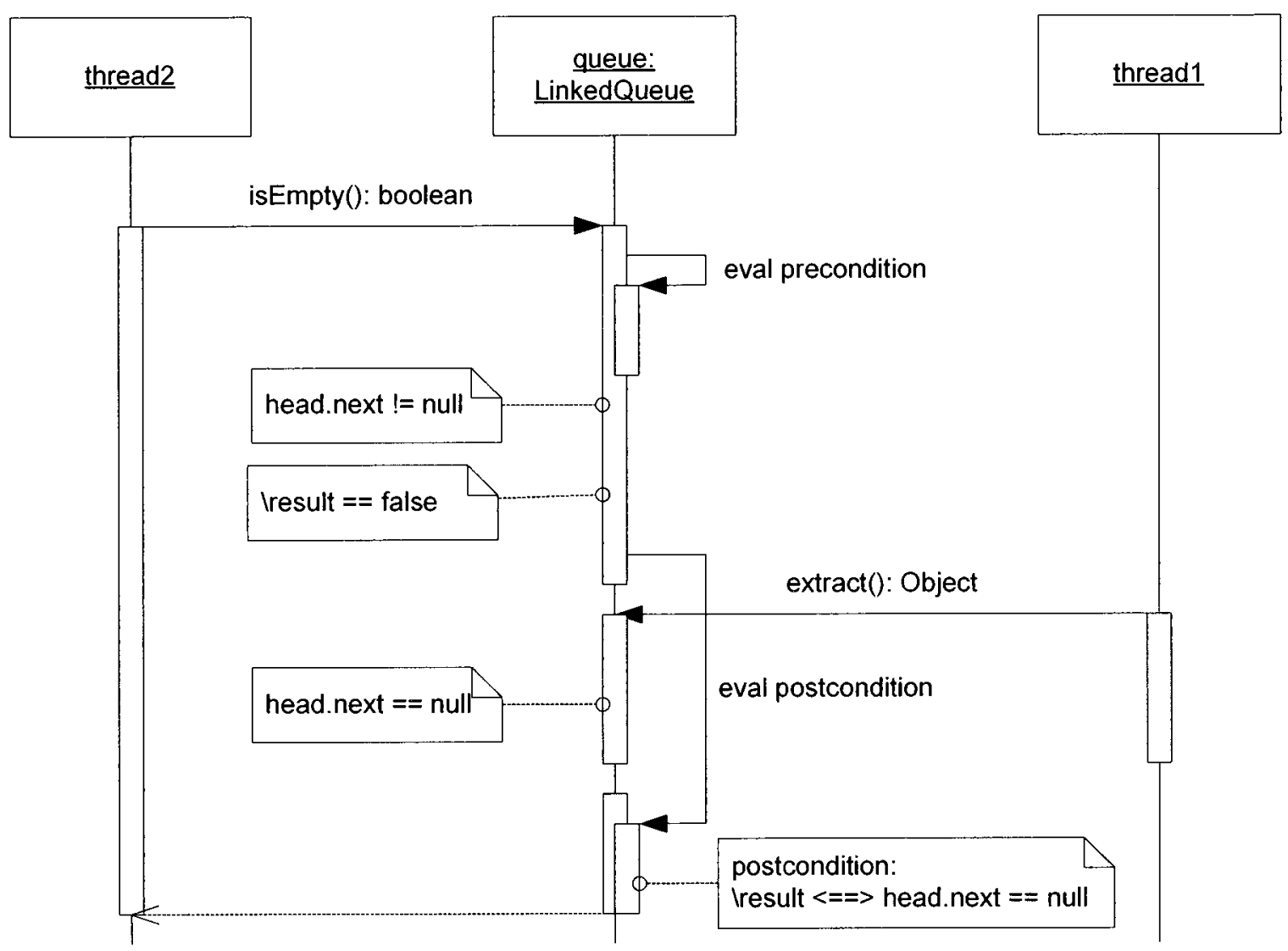

Figure 2: Sequence diagram depicting the problem of external interference.

Two threads accessing the same instance of Linkedqueue, one executing the method extract () from Listing 1 (thread 1) and another executing the method isEmpty() from Listing 2 (thread 2), interfere with each other if thread 2 executes isEmpty() completely (i.e. it returns) but is pre-empted before resuming to the client (i.e. before the method's post-state) and thread 1 executes extract() completely. If this queue has only one element before both threads started executing, isEmpty() returns false, since it perceives the element in the queue. After extract() executes, the queue becomes empty. Once thread 2 resumes, it evaluates the postcondition of isEmpty () in its poststate. At this point, the queue is empty and the postcondition formula evaluates true. 
This example can be seen on Figure 2. We represent the "instantaneous" execution of extract () by not drawing a return arrow. The problem in this case is localized on the evaluation of the postcondition. Once isEmpty () returns false, thread 2 is pre-empted before executing the assertion checking code in the post-state and extract () executes completely, which results in setting head. next to null. Once thread 2 resumes, the postcondition evaluation code perceives the value of head.next set by extract(), not the one at the point is Empty () returned. This causes the postcondition to evaluate false, producing a false negative.

External interference breaks the Hoare-style reasoning by making observable changes between a method execution and the evaluation of the associated assertion formula, either for pre or postconditions.

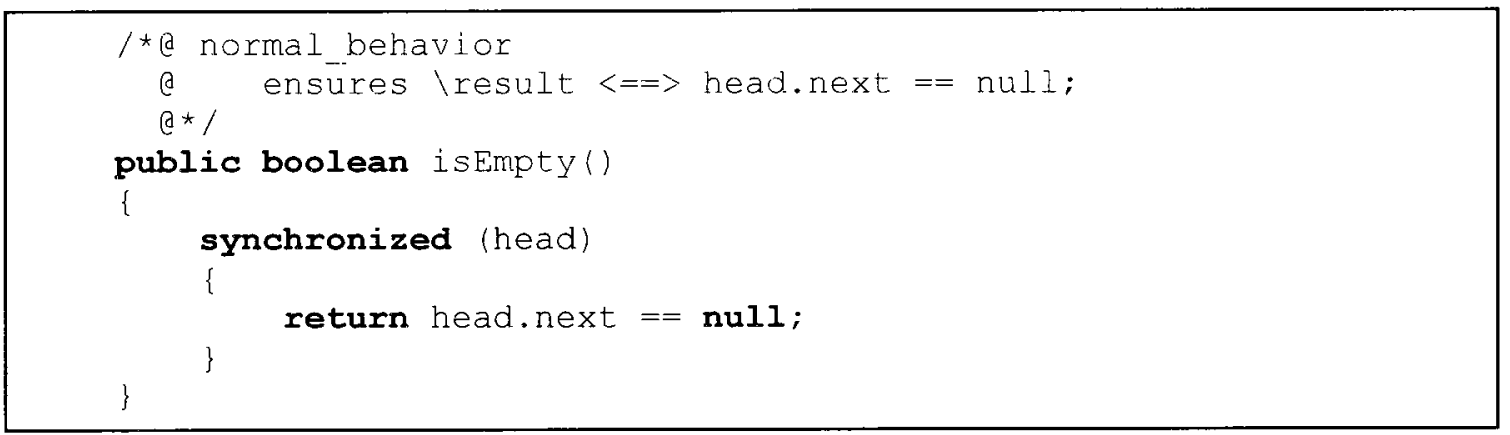

\section{Listing 2: JML specification of method isEmpty () of class LinkedQueue.}

A closer look at the contract in Listing 2 tells that the reason why external interference is a problem for this particular case is because the ensures clause (the postcondition) makes reference to the internal state of the object, which can be concurrently modified by other threads. Once the current thread reaches the post-state of method isEmpty() it no longer has a lock on the relevant object for this contract, namely the object pointed to by the field head. One must also note that the precondition for this method is true, i.e. the client has no obligations. A similar case happens on Listing 1 for the preconditions. They reference the private field head and last and their fields through the \old expressions in the postcondition clauses. Although located in the ensures clause, the values of the $\backslash 01 \mathrm{~d}$ expressions are computed in the method pre-state to be later referenced in the post-state 
while evaluating the postconditions. One should also note that the effective precondition of this method is true. The specification cases (conjoined by the keyword also) simply specify the different behaviours of the method depending on the queue being empty or not. They do not impose any obligations onto the client, which is allowed to call extract () whether or not the queue is empty.

The issue of information hiding and modular reasoning for concurrent contracts is left for a later section. At this point, the focus is on the fact that threads interleaving cause problems to concurrent contracts because the evaluation of such contracts leads to access to unprotected data in the method pre- and post-states. Another way of looking at it is to notice that the time the method observes these values (e.g. in an if statement) is different from the time the contract observes them. In other words, "stuff" happens between the point a thread observes a variable on a contract and the same thread observes the same variable in the method, and what happens is not under the control of the observer thread. Our solution to this problem (presented on the next chapter) is to make sure the contract and the method observe the same values at the same time.

\subsection{Specification Inheritance}

Specifications can be inherited from interfaces and super-classes. The example in this section makes use of an interface to illustrate the issues with data abstraction and concurrency. The issues are the same in the case of a super-class. Specification inheritance is a form of implementing behavioural subtyping [8].

Listing 3 shows the specification of the Channel interface. It declares two model fields. A model field (a field with the model modifier) is a field that does not have to be implemented but can be used in a specification as any other field. The "value" of a model field can be defined in implementing classes or, in the case of being declared on a class, by concrete fields of such class or its subclasses. Both fields in this example are marked instance, meaning that they are fields of the object implementing the interface instead of static fields of the interface. Java only allows static final fields on interfaces. JML 
allows both static and instance fields on interfaces. They can only be used inside specifications, obviously.

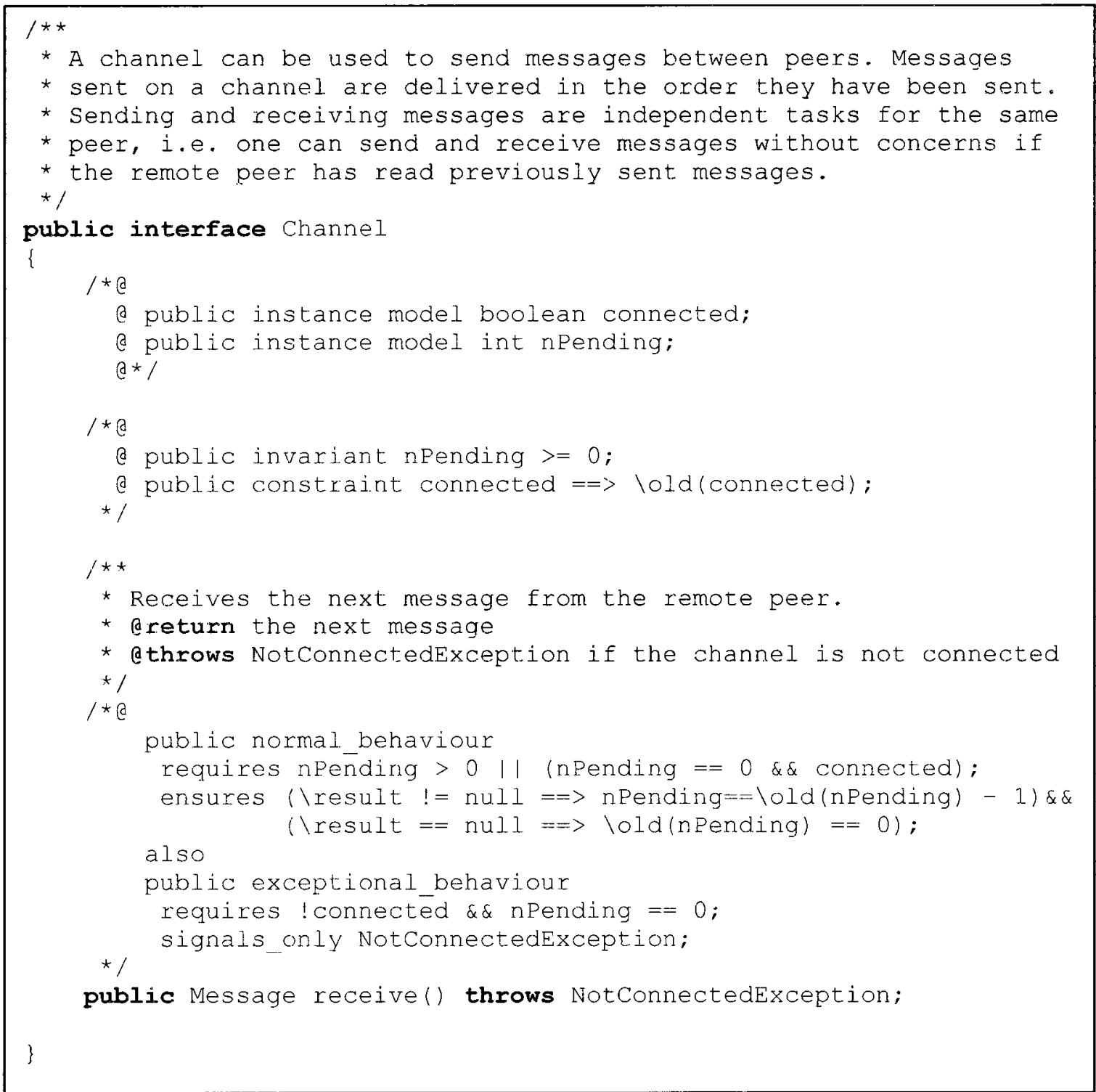

\section{Listing 3: JML specification of interface Channel. Only the receive method and relevant model fields are shown for simplicity.}

Interface Channel can be implemented with the help of a Pipe (from Listing 5), as shown by class PipedChannel in Listing 4 . The represents clause maps the value of a model field to an expression based on concrete fields of the class. The model field connected obtains its value from the concrete fields closed and remoteclosed according to the 
Boolean expression !closed \&\& !remoteclosed. The value for the model field nPending comes from the input pipe in, via its getsize() method.

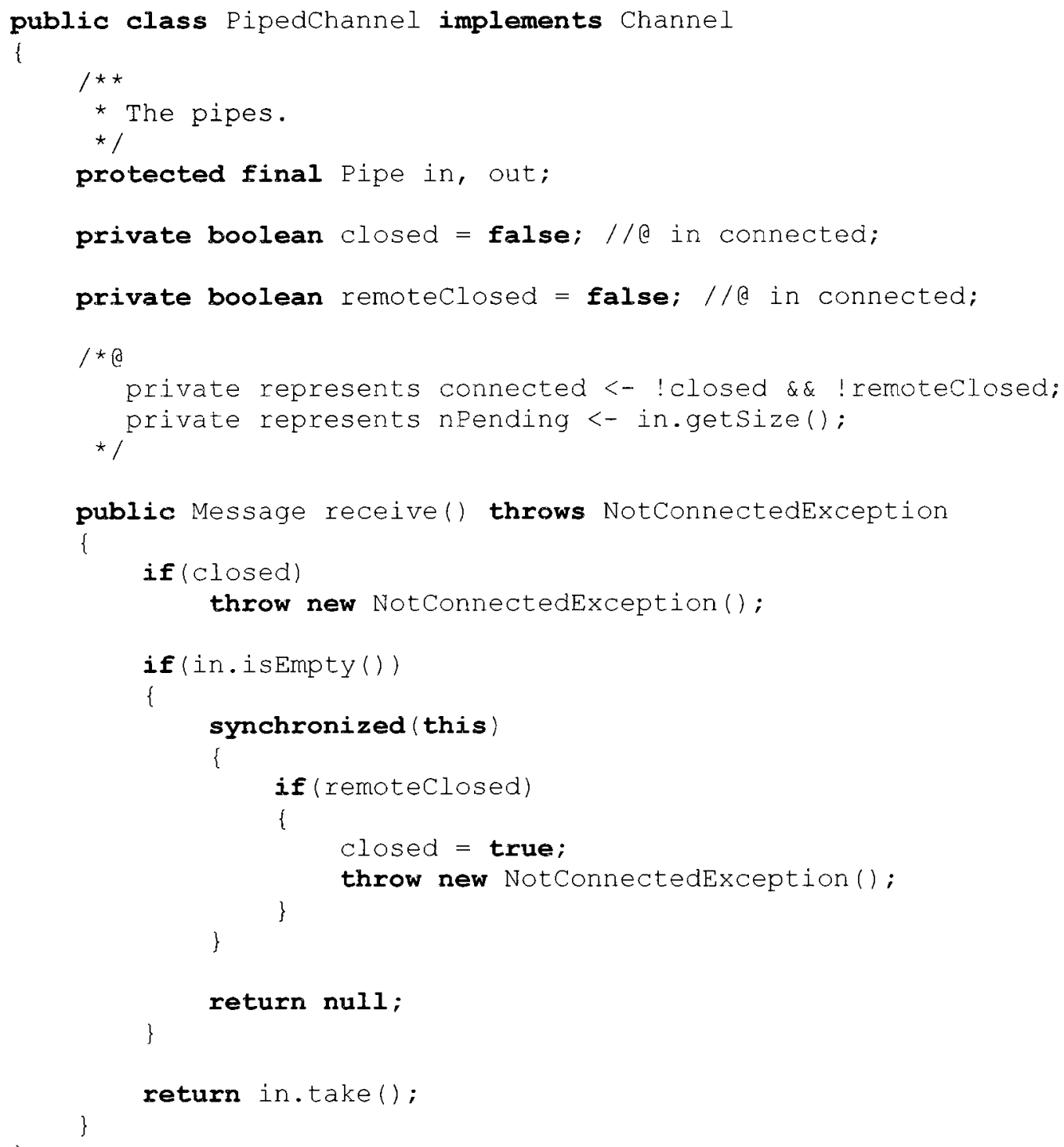

Listing 4: JML specification of class PipedChannel. Only relevant methods, fields and specifications are shown for simplicity.

Since method receive() in Listing 4 does not declare any specifications, it inherits the parent's specification without any changes. The inherited normal specification (the one that specifies the conditions for the method to return normally) in Listing 3 simply says that receive() will return any Message objects the channel contains even if it has 
already been closed or null if it is empty. The exceptional specification states that receive() will throw a NotconnectedException if the channel has been closed and it is empty.

The problem with this case is very similar to the previous ones: interference causes preand postconditions to be evaluated at unsafe points, since they reference the object's internal state. The difference is that such internal state is made visible through model fields in the interface specification. Although one might argue that such specification is improper for a concurrent environment because it was not designed with concurrency in mind, nothing on the interface states that it actually is supposed to be used only in a sequential environment. One might implement it sequentially or concurrently offering the same guarantees.

The issue here is more subtle than on the previous cases. Model fields are used to specify behaviour without giving out implementation details. They can be seen as mathematical entities on which it is safe to reason about the behaviour of the object. Model fields do not have storage, i.e. their values are derived from the container object's state, not through direct assignment, which is forbidden. In the examples above, one can see that the value for nPending comes from the evaluation of the getsize() method of the field in. One can think of a model field as a read-only field that is modified outside of the specification control. Apart from just allowing abstract modelling [20, 21], model fields play a vital role in information hiding, modular reasoning and behavioural subtyping [12]. Thus, in comparison with the examples on previous sections, the contracts on this example are not accessing internal data directly; they are making use of the available modelling facilities to best describe the behaviour of the object in question.

A solution to the issue of contracts for concurrent objects must accommodate the use of model fields. Current solutions do not even acknowledge the existence of a problem. One approach to the problem is to require the client to guarantee thread-safety of all fields referenced in method specifications [22, 23]. Apart from other issues related to concurrency control we discuss in section 4 , this solution cannot be applied to model fields at all. Clients do not have visibility on how the provider realizes model fields and 
therefore cannot know which locks to acquire. Furthermore, if the client only sees the interface, it might not even know that there are concurrency control issues. The problem presented by verifying abstract specifications in a concurrent environment is then that, in general, the client cannot be required to perform additional concurrency control simply to guarantee thread-safe access to fields present in such specifications because they do not present any concurrency control related predicates or because the specification refers to model fields.

\subsection{Blocking Behaviour}

Java implements blocking behaviour through the use of the wait() primitive on a monitor on which the executing thread ${ }^{3}$ holds a lock [11]. The use of such feature usually follows the pattern of a guard condition that must be satisfied for the method to proceed executing. While such condition is not satisfied, the method blocks execution of the current thread, releasing its lock on the target monitor object. Upon a state change, other threads notify the waiting thread(s) via the notify() or notifyAll() primitives of the monitor lock, upon which time a waiting thread wakes up, acquires the lock on the monitor object and rechecks the guard condition. If it is satisfied, the method proceeds executing. If not, it blocks again.

This general pattern derives from monitors according to Hoare [23]. A monitor contains private data, condition variables and procedures to acquire and release it. A condition variable offers the operations wait(), which causes the executing thread to block and relinquish its lock on the monitor, and signal (), which causes a blocked thread to wake up and start executing as soon as it obtains a lock on the monitor. The monitor acquisition operation checks a wait condition (a predicate based on the monitor's private data) to decide if it should wait or if it should proceed with the acquisition. The wait condition must be satisfied for the monitor acquisition to proceed. Once satisfied, the wait condition can (and likely will) be invalidated by the acquisition procedure. Therefore, a wait condition is only guaranteed to be valid at a particular point inside the acquisition

\footnotetext{
${ }^{3}$ In Java terminology, the executing thread is also known as the current thread. These terms are used interchangeably throughout this text.
} 
procedure. After such point, the acquisition procedure executes without waiting. Since a monitor is equivalent to a semaphore (i.e. one primitive can be used to implement the other) our presentation based on Java is valid for any other object-oriented language that uses either monitors or semaphores as the mutual exclusion primitive.

JML makes use of the when clause to specify wait conditions [16]. The when clause specifies a predicate that must be satisfied at a particular point of a method execution after which the executing thread is not allowed to block. Such point is called the commit point (from the notion of commit atomicity) and is marked with the JML label commit inside the method. Furthermore, the specified predicate is only required to hold at the commit point; nothing is said regarding its validity afterwards. Other threads or even the current thread are allowed to invalidate it after the commit point.

Listing 5 shows an example of the use of the when clause. Method get () will block until the pipe is not empty, after which it will return a non-null message. If the pipe is closed, however, it will return null. This contract is quite simple but nevertheless presents the problem of external interference due to thread interleaving with respect to the closed field. 


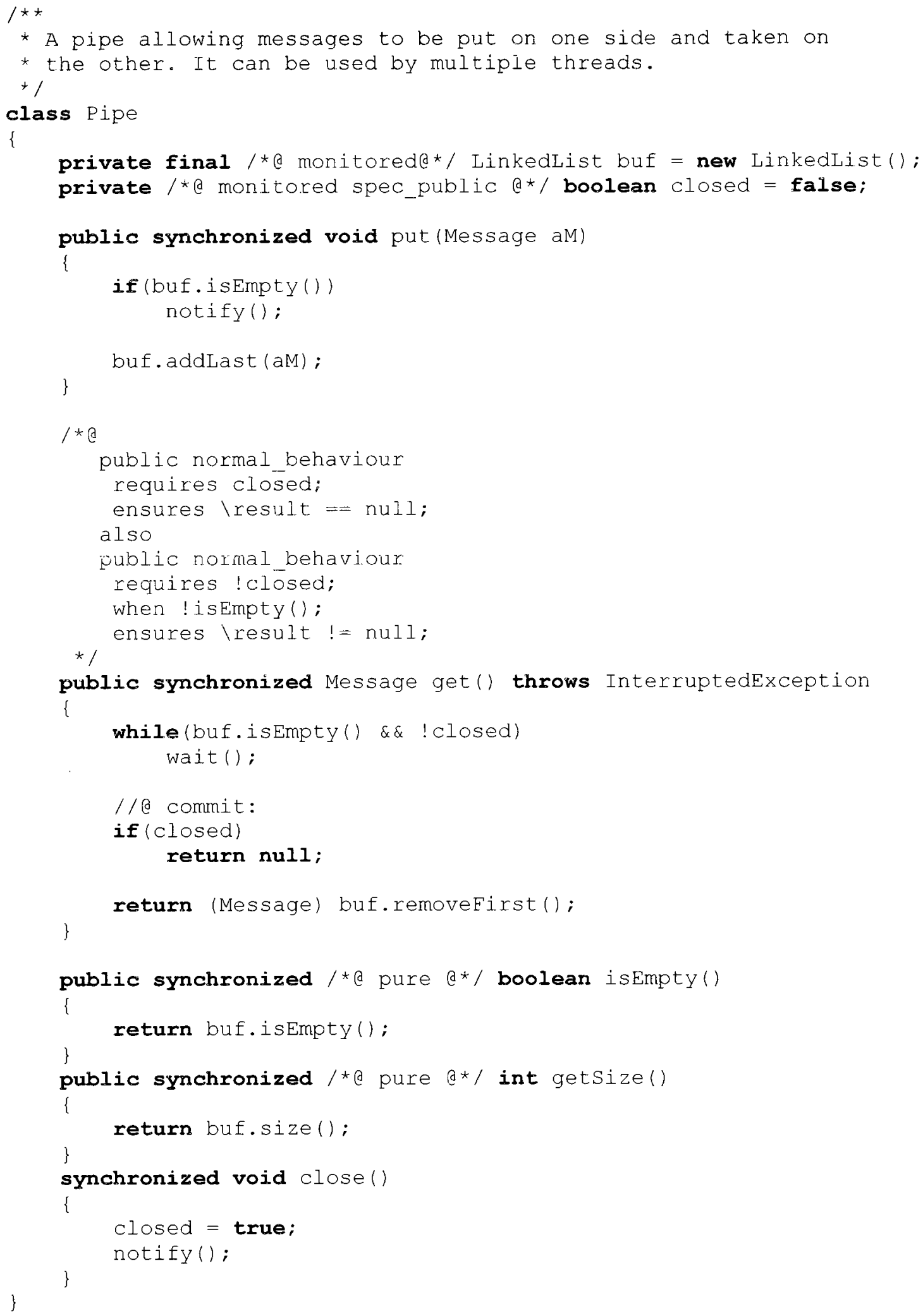

Listing 5: The class Pipe and its JML specification. 
The interesting point of this example is that there are interference issues regarding the evaluation of the wait condition. The commit point demarcates the location where the predicate can be safely evaluated. However, if external interference caused closed to be seen as false in the pre-state but true inside the method, the wrong wait condition would be evaluated (a missing when clause has a default value of true) $[13]^{4}$ since the second specification case would be selected instead of the first.

The issue of this example is, again, the fact that specification cases observe internal state without acquiring the proper locks. In this particular example it is even clearer than on the previous one. The field closed has the monitored modifier, which states that accesses to it must be performed while holding a monitor lock on the enclosing object. Thus, for the precondition to refer to it while on the pre-state would already constitute a violation from the perspective of runtime assertion checking.

The problem of internal interference is implicitly dealt with in [16] since methods are expected to be atomic. Atomicity, however, does not guarantee absence of external interference. It only guarantees that the method can be treated as if it was executing sequentially from a reasoning point of view. To implement runtime assertion checking of such method requires the same measures required for a non-atomic method.

Combining blocking behaviour with specification inheritance yields a unique problem Class Blockingchanne12, in Listing 6, illustrates this situation. It extends class PipedChannel (from Listing 4) to add blocking behaviour to the receive() method. The JML specification also shows an additional specification case for this method. The keyword also in the beginning of a method specification denotes inheritances: the resulting method specification is the combination of the one seen on this class and the

\footnotetext{
${ }^{4}$ This is the case in this example due to the use of the normal_behaviour keyword, which marks a specification as heavyweight. Heavyweight specifications have well defined default values for omitted clauses, as opposed to lightweight specifications, in which omitted clauses have a default value of $\backslash$ not_specified. The default values for heavyweight specifications including more details about them can be found in the reference.

${ }^{5}$ An extending method specification must always start with the keyword also. It is not an option for a method specification to not inherit its supertypes' specifications.
} 
one on the Channel interface (the superclass PipedChannel does not add anything, in this example).

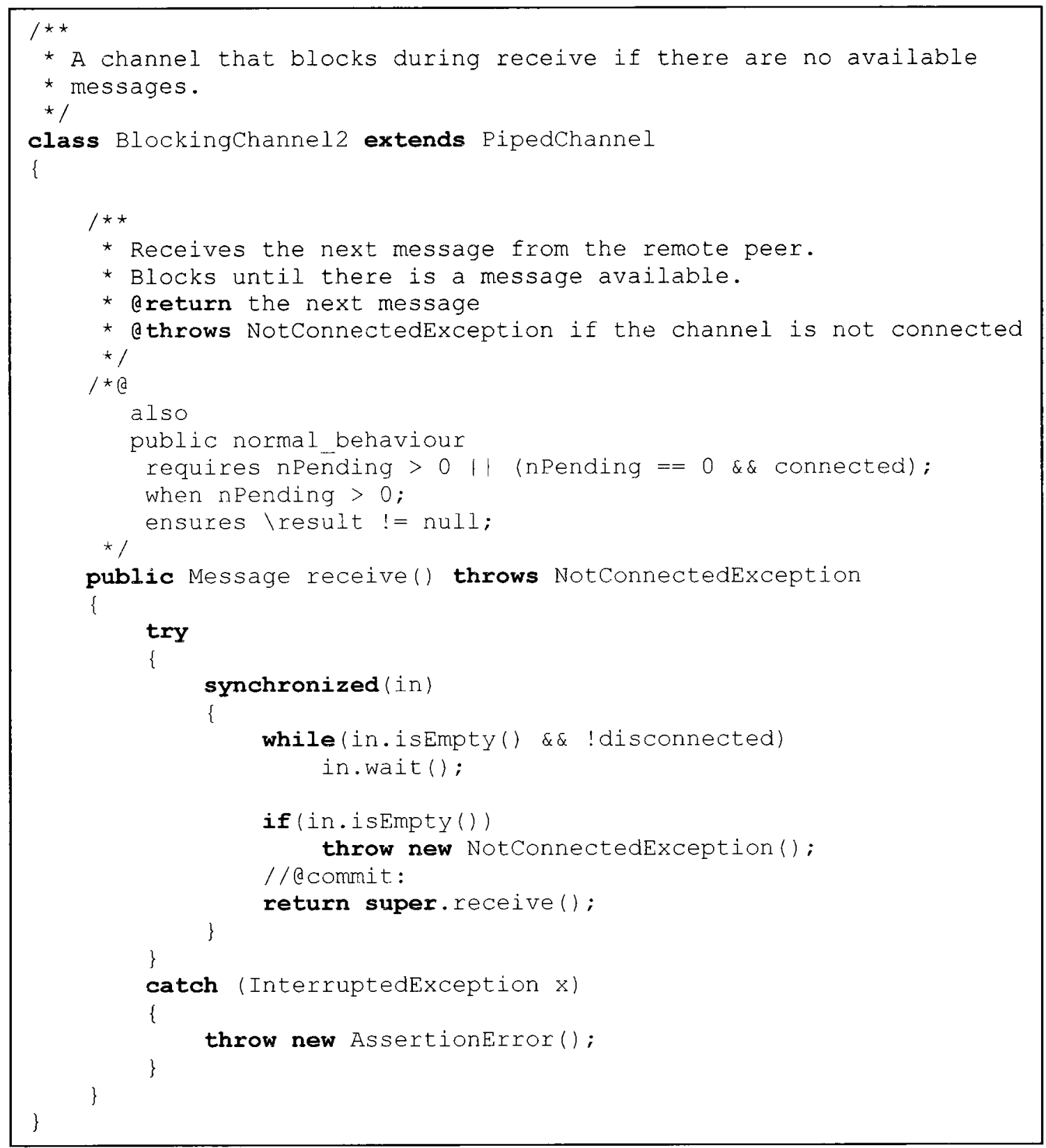

\section{Listing 6: JML specification of class BlockingChannel2. Irrelevant methods and fields are omitted for clarity.}

JML enforces behavioural subtyping. The resulting method specification works as if this method's specifications from all super-types were copied to the current type and joined 
by the keyword also into specification cases. Specification cases are equivalent to a single specification case in which all preconditions are disjoined and the postcondition is the conjunction of all the implications of each case precondition to its postcondition. Listing 7 shows two equivalent method specifications: the first, for method $m()$ with specification cases and the second, for method $\mathrm{m} 2()$, without. The complete desugaring process for all JML clauses and constructs can be found in $[7]^{6}$.

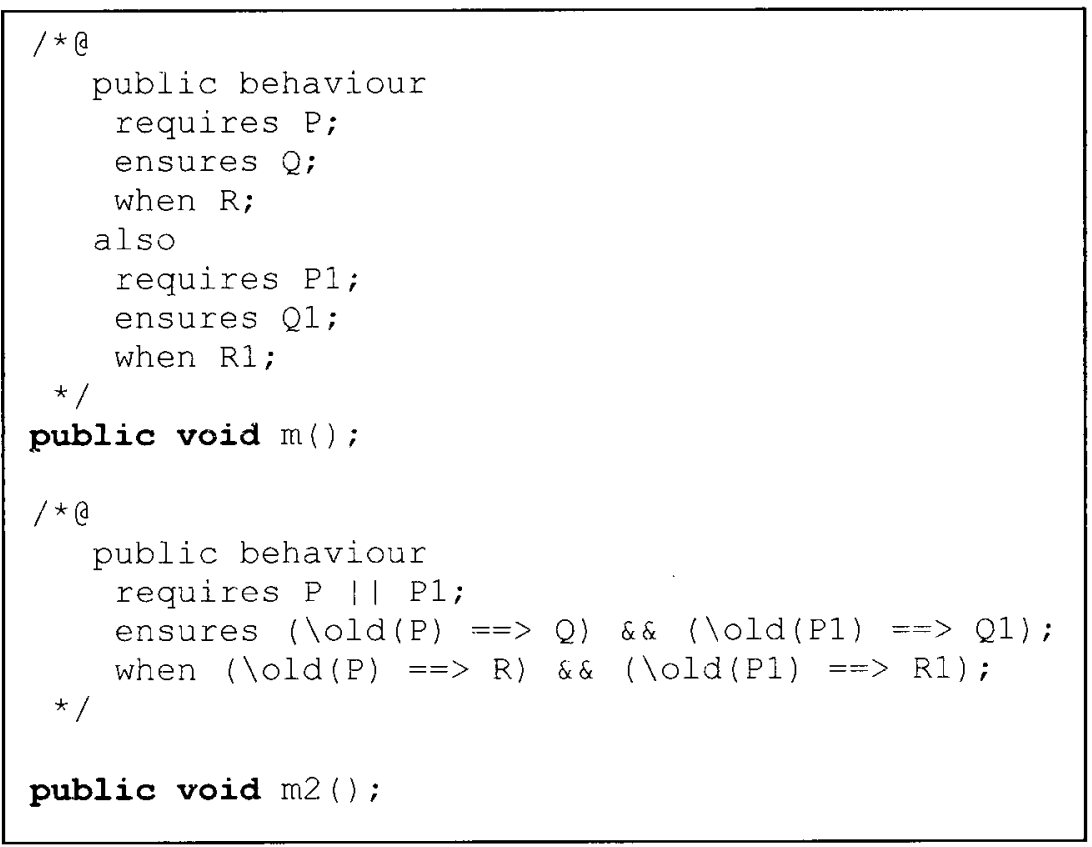

\section{Listing 7: Example of desugaring of method specifications. The two method specifications are equivalent. $P, P 1, Q$, and $Q 1$ represent JML predicates.}

The resulting specification for Blockingchannel2.receive() contains the problems discussed in the previous section. It also contains the problems due to external interference discussed in section 2.5. It contains yet another problem resulting from the addition of blocking behaviour to a sub-type's method: the parent's specification becomes invalid. Looking at just the local specification of receive() in Listing 6, one should note that the only guarantee this method offers is that it will return a non-null value. This is due to the blocking behaviour. The when clause guarantees that the method

\footnotetext{
${ }^{6}$ The JML literature uses the term effective specification to designate the result of the desugaring process. The term effective specification has a slightly different meaning in this work. It means the desugared specification in which all the predicates have been minimized.
} 
will block until there are messages available to be received but it does not say how many. While a client blocks waiting for a message to arrive, any number of messages might be put into the pipe. This breaks the contract on the Channel interface, which specifies that the number of pending messages after the method executes will be one less than what it had prior to its execution.

Simply put, adding blocking behaviour to a method likely breaks its inherited specifications, i.e. it causes it not to be a behavioural subtype. This is not the whole truth, though. Setting the issue of interference aside for a moment, one can notice that if the thread does not actually block during the method execution, the contracts can be honoured. Blockingchannel2.receive() is an example of this case. Since the thread holds a lock on the field in while calling the superclass' implementation of the method, there is no possibility of interference, and thus all contracts are satisfied.

This apparent problem of the JML semantics (or of behavioural subtyping in general) needs further discussion. The issue here is caused by the inherent nature of blocking. Once a thread blocks, it releases its lock on the monitor on which it blocks to allow other threads access to it so they can modify the state up to the point the wait condition can be satisfied and the blocking thread can proceed executing. Looking at the contracts for receive() in Listing 3 and Listing 6 one should note that both preconditions for which the method returns normally are identical. One should also note that both contracts are satisfied if the wait condition nPending $>0$ is satisfied in the pre-state. The problem occurs in the case the wait condition is not satisfied in the pre-state, i.e. if the pipe is empty. This causes the current thread to block until it is satisfied. In a sense, this particular specification case is negating its own precondition and by doing so it is satisfying the precondition of the other specification case. But since lold(nPending) was computed in the pre-state instead of reflecting the value of $n$ Pending at the commit point, the postcondition fails to be satisfied. The problem here is not with behavioural subtyping but with the contracts themselves: the wait condition refers to a predicate present in the precondition that may not have been satisfied at precondition evaluation time, there is more than one specification case with satisfying preconditions, and the satisfaction of the wait condition implies the satisfaction of the other case's postcondition 
if the value of $\backslash o l d$ variables were recomputed at the commit point. This situation causes the subtype to not be a behavioural subtype because the implementation will not be able to satisfy the specification in all possible interleavings.

\subsection{Locking Policies}

The specification of locking policies is one of the strategies to guarantee deadlock freedom in concurrent software [15]. Locking policies can control many aspects of the locking acquisition and release process. In this work we restrict ourselves to the specification of lock acquisition order (from the language standpoint). Lock ordering works as a building block to specifying more complex policies.

The intent of specifying lock acquisition order policies is to define conditions regarding the order in which multiple threads can safely acquire locks. By abiding to such conditions, threads are guaranteed to never deadlock, assuming that such conditions are specified correctly. Typical policies one wants to specify are "no locks should be acquired after acquiring a lock on an instance of this class" or "acquiring a lock on a tree node requires the acquisition of a lock on its parent". The aim of this section is to describe the issues with the current approach of specifying such conditions in the context of runtime assertion checking.

JML overloads the operators $<$ and $<=$ when applied to objects to evaluate the order the current thread acquired the locks given as arguments. A lock object 11 is smaller than another 12 if the current thread acquired 11 before having acquired 12. JML also provides the \max expression, which returns the largest of a set of objects according to the above definition of $<$.

The current way to specify lock ordering policies uses the axiom statement (see section 2.7.2 of [19]). Axioms are predicates that can be specified inside a class declaration. They can be used by theorem provers for correctness verification. The RAC ignores axioms [14]. The use of axioms to specify lock ordering properties presents several disadvantages. First, they are not part of class invariants and, thus, are not part of a contract. Although the semantics of axioms states that it should be valid at the beginning 
of every method execution, it does not say anything with respect to its validity at the end of such execution. Hence, it cannot be considered a class invariant. Second, axioms accept a general predicate as an argument. Although flexible, this can lead to several reasoning complications that are not considered worthwhile to tackle (e.g. having to use theorem proving to analyze the predicates). Third, it is possible, although infrequent, to produce undesirable specifications. This is illustrated by an example from section 2.7 .2 of [19] and shown in Figure 3 through an object diagram. An axiom stating that locks organized in a binary tree must be acquired prior to acquiring any of its children is inconsistent in the presence of tree mutation. This happens because the axiom is evaluated at the beginning of the method, i.e. the permissible locking orders are computed then. If the tree is mutated by changing its configuration, even if following the locking axioms, the resulting tree could have nodes that are in reverse position compared to the original tree, i.e. a parent could become a child of its original child. If a second modification were to happen inside the same method an attempt to follow the locking policy (acquiring the lock on the parent first and then on the child) would cause a violation to be reported. In this example, if the tree in Figure 3 moves from its initial state to its mutated state and back inside the same method while respecting the locking policy, the axiom in question would falsely report a violation upon acquisition of $\mathrm{v}$ after $\mathrm{w}$ since, originally, $v$ was the parent and $w$ the child. This happens because the axiom binds any free variables at the beginning of the method only and that is what dictates all acceptable orderings. During the reversion process, although $w$ is then the parent of $v$, the binding $v$ $<w$ is still in effect, thus causing a violation to be reported. 

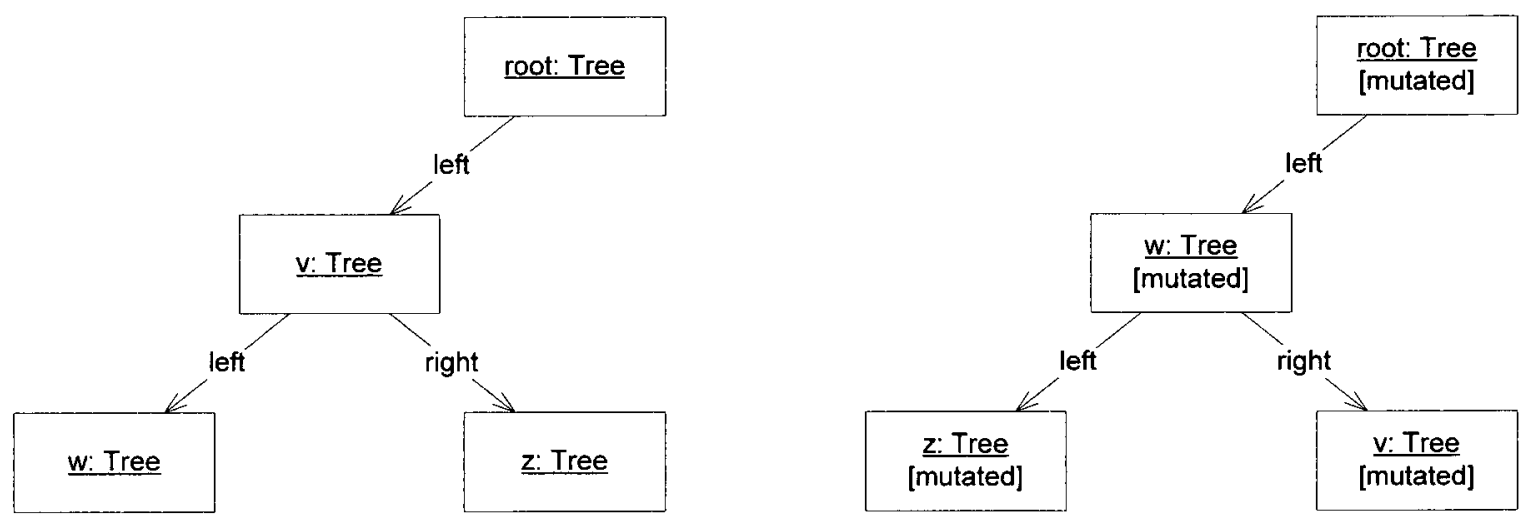

Figure 3: Object diagram showing the two states of the tree. The mutated state depicts a rotation of the nodes from the initial state.

The three issues above call for a better solution, which we present in section 3.4. The first issue precludes treating locking policies as part of a contract since it fails to provide a guarantee on the state subsequent to a method execution. The second issue affects the feasibility of implementing such checking during runtime in an efficient manner since such predicates would have to be evaluated at every step of the method execution due to their subject not being restricted to lock acquisition order. The third issue affects the correctness of the results.

\subsection{Thread Safety}

The core idea behind thread safety is one of non-interference [16]. Thread safety can be achieved in a variety of ways. They all relate to the way data can be accessed. Data that is local to a thread (i.e. not visible to other threads) is not subject to any interference issues. Access to shared data (i.e. visible to multiple threads) must be protected by a lock. By doing so, one guarantees the absence of interference when accessing such data. Aliasing and ownership patterns that exist among objects also determine if accesses to a certain object are thread-safe or not. For instance, an object that is created inside a method and then returned to its client can be safely accessed if one knows that the creator class did not keep a reference to it (i.e. it is not part of the provider object representation). Serializability criteria for a region of code can be used to establish if the execution of the statements of a method in the presence of concurrency (i.e. possibly interleaved with 
statements executed by other threads) is equivalent to a sequential execution. A method is said to be atomic if the statements in the method body are serializable. The atomicity of a method execution ensures that sequential reasoning can be applied to it, i.e. it can be considered as executing in a single atomic step.

JML provides several constructs to specify all the above aspects of thread safety [16] ${ }^{7}$. The expression \thread_local states that its argument is owned by the current thread, i.e. it can only be reached by this thread. The monitors_for clause, in the format monitors_for ident <- store-ref-list, specify that all non-null locks specified in storeref-list must be held prior to accessing ident. A simplified version of this clause is the monitored field modifier (see Listing 5 for an example), which states that a lock on the object containing the field instance, i.e. this, must be held prior to accessing the field. A stronger construct is the $\backslash$ lock_protected predicate, which states that access to the object provided as argument is protected by a non-empty set of locks, although it does not specify which locks in particular. The thread_safe predicate is defined as the disjunction between \thread_local and \lock_protected, i.e. an object is considered thread-safe if it is either local to a thread or access to it is protected by a lock.

JML provides a number of constructs to deal with locks explicitly [16]. The \lockset expression refers to the set of locks held by the current thread. The locks ${ }^{8}$ clause in a contract specifies which locks a method acquires and releases during its execution. A method is not allowed to acquire more locks than what is present in the locks clause for the applicable specification case. The contract for Pipe.get() can be extended to include this clause as shown in Listing 8. Thus, it needs to be present in all cases for which it is relevant. The \lockset expression is of JMLObjectset type. This is one of many modelling types provided by JML [12]. It is a set to which membership is based on object identity. Like other modelling types, its objects are immutable. One can obtain subsets, iterate over its contents, check membership, etc. but it cannot be modified.

\footnotetext{
${ }^{7}$ Although described in the reference, the following constructs have not yet been implemented on the JML checker or on the JML compiler (RAC), as of release 5.4 of the common JML tools: \thread_safe, $\backslash$ thread_local, \lock_protected, \independent, and the atomic modifier. The 5.4 release happened on February $8^{\text {th }}, 2007$.

${ }^{8}$ Although described in the referred article, the locks clause has not yet been implemented on the JML checker or on the JML compiler (RAC), as of release 5.4 of the JML tools.
} 
There are situations in which it is useful to leave to the client the task of acquiring the necessary locks prior to executing a particular operation. This usually happens in cases the provider object is aware of concurrency issues but a per-operation concurrency control is not meaningful or practical. In such cases, concurrency control is delegated to another object (or set of objects) and it is the client's responsibility to acquire the locks from such object prior to invoking the desired operation or set of operations. It is then easier to specify such behaviour directly in terms of locking requirements than through thread-safety predicates. A typical example of this case happens in transaction processing. The client must obtain a transaction lock prior to executing a set of operations on a set of objects and release it in the end. The operations performed while holding such lock are executed atomically. It does not make sense for the operations themselves to acquire the transaction lock but they assume they must be executed in the context of a transaction. Another example in which it is easier to specify locking requirements than thread-safety predicates is when one uses well-known coarse grained locks to protect a set of objects. It is then very clear that a client must acquire a particular lock before executing a particular operation.

Aliasing control is done via the Universe type system embedded in JML. By using the rep modifier, one can specify that a particular object on a field declaration is part of the representation of another. This means that there should be no way to reach such an object from outside the enclosing object. The readonly modifier prevents that the object pointed to by a reference be modified through it.

The atomic modifier can be applied to methods and classes. It flags that a method or a class is atomic. An atomic class is one in which all its methods are atomic. The atomic modifier is not inherited by subclasses.

These constructs were devised having static checking, theorem proving or even modelchecking as the target verification techniques $[18,24]^{9}$. There are several challenges in adapting them to runtime assertion checking. These can be summarized as the impossibility (or at least infeasibility) of stopping all threads of execution to evaluate

\footnotetext{
${ }^{9}$ Method atomicity can also be checked using dynamic techniques.
} 
reachability predicates. For instance, to decide if an object satisfies the predicate $\backslash$ thread_local, one would have to compute all references to such object and make sure they are all rooted on the same (the current) thread. Evaluating the \lock_protected predicate presents a similar challenge. With the monitors_for clause, things are simpler for the common cases, but as complex in general. If modular reasoning is possible, i.e. the visibility of the monitored field is restricted (private), it is just a matter of inserting code (instrumenting) to check if the required locks have been acquired by the current thread, as long as there is no representation exposure. In such case, or if the field is accessible, external accesses to the referred object cannot be controlled by the container class, so such predicates would have to be constantly monitored or simply not at all.

Atomicity is in a different category. The techniques described in $[16,18,24]$ use purely static reasoning, purely dynamic techniques or both, respectively. None of them can be easily adapted to a RAC scenario in which a predicate can be simply evaluated at the beginning or end of a method execution and a decision reached regarding its atomicity. That is because the atomicity checking algorithms rely on annotations provided by the programmer to determine the set of locks protecting access to a variable (which is possibly flawed) or the lock inference algorithms used in their place require multiple executions of a method (or block of code in general) being checked for atomicity to make a determination. The atomicity modifier is thus more of a language statement than a predicate to be checked. It simply informs that sequential reasoning can be used for the method in question. Since the focus of this research is on RAC, no atomicity related construct will be further considered.

The challenges presented above may limit the implementation of such constructs in a RAC compiler but the fundamental problems are the evaluation point and their use in the presence of specification inheritance. The identification of these challenges and problems is one of the contributions of this work.

Locking requirements are specified through the use of the \lockset construct and checking for membership of a particular object in this set. Thread-safety is specified via the \thread_safe predicate on an object. Such predicates are currently specified in the precondition of a method. This presents two major problems. The first problem is the 
evaluation point. $\mathrm{DbC}$ specifies that preconditions be evaluated prior to the first statement of the target method. In JML, this is done in the pre-state, before entering the method but after binding the parameters. It could have been done, however, as the first statement of the method. There is no difference between these approaches for sequential programs. For concurrent programs, however, there are. The first statement of a method might already be protected by a monitor lock (synchronized methods in Java). Take the method get () of class Pipe (Listing 8). It specifies a precondition containing locking requirements and a functional requirement. This implies that not only interference has an influence on the determination of the locking requirements to evaluate pre- and postconditions, but also the fact that obtaining such locks (whatever the strategy) might affect the evaluation of the corresponding predicate. For instance, suppose one decides to evaluate the precondition of the method get () in the method pre-state. In this case, it is subject to external interference because it does not hold a lock on this, which protects the access to the field closed from race conditions. If, however, the evaluation happens just before the first statement of the method, i.e. right after acquiring the lock on this but before executing the while statement, interference is not a problem but the term ! lockset.has (this) will evaluate false, which is not the desired behaviour. 


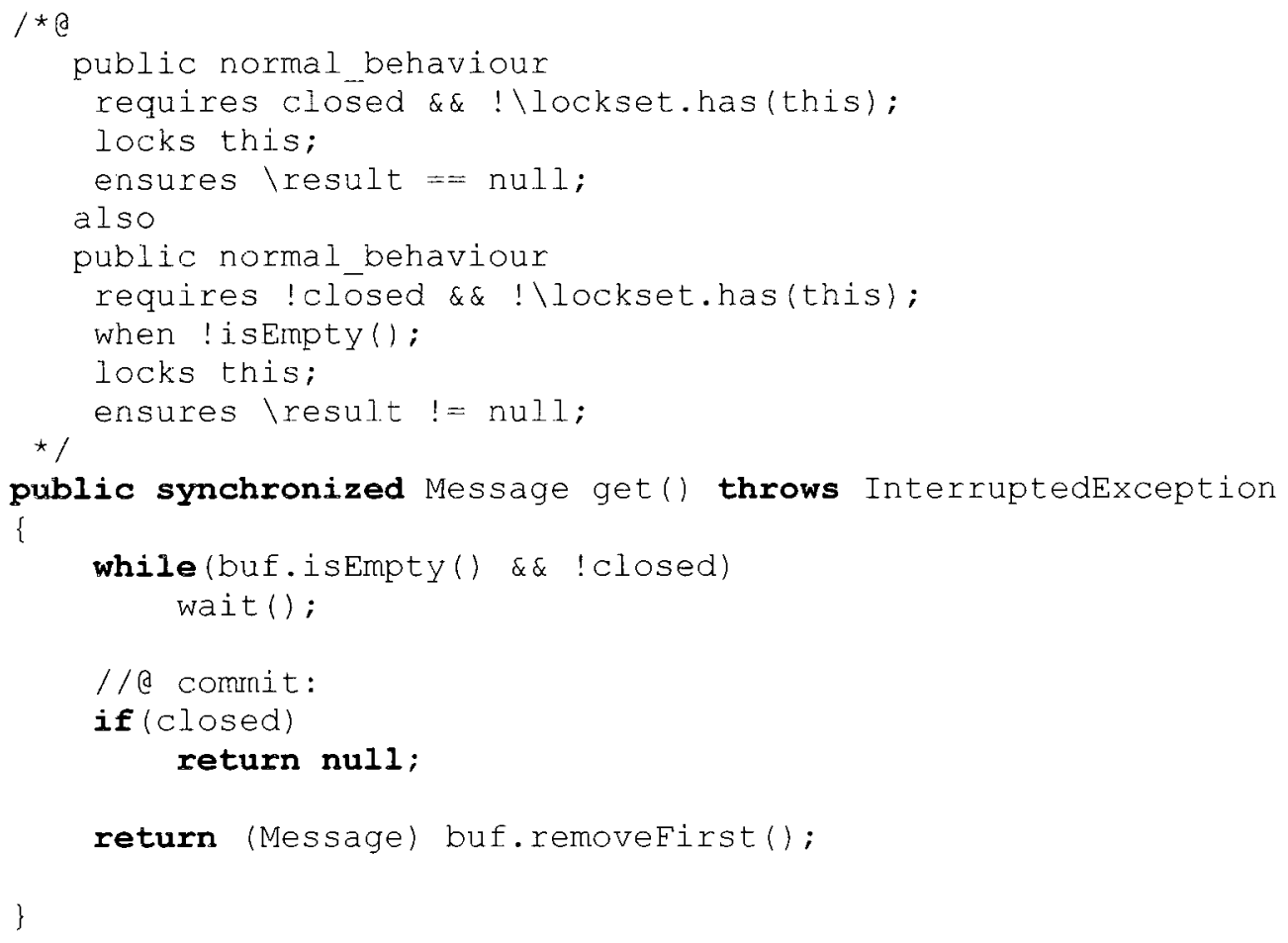

\section{Listing 8: The extended contract for the get ( ) method of the class Pipe.}

Locking requirements specifying that a particular lock is not held by the current thread will likely not hold even if they were true in the pre-state. The $\backslash$ thread_safe ${ }^{10}$ predicate presents the reverse problem. It is possible for an object (e.g. a method argument) to not be thread-safe in the pre-state but be thread-safe inside the method due to the protection created by the monitor lock. One should note that the validity of the effective precondition does not change if evaluated in the pre-state or inside the method. This is the first evidence that locking requirements and thread-safety specifications do not belong in the precondition. The ability to move the evaluation point of pre- and postconditions is essential to our solution as described in section 3.1 .

The second problem is related to specification inheritance and behavioural subtyping. A predicate involving locking requirements or thread-safety is not required to be satisfied in a sub-type as long as the inherited specifications are satisfied. This is due to the fact that

10 The Ithread_safe predicate is defined as the disjunction of the $\backslash$ thread_local and $\backslash$ lock_protected predicates. Our discussion assumes the case in which \thread_local is not satisfied since its evaluation is not affected by the evaluation point. 
the effective precondition of a method is the disjunction of all inherited preconditions with the precondition of the target type. If that was not the case behavioural subtyping would be violated. The reverse case can also happen. A sub-type might weaken the precondition in a way that inherited locking requirements and thread safety specifications are not required to hold. This is the main point for not having these properties in the precondition. An analogous argumentation can be made for postconditions.

The main point is that preconditions, postconditions and invariants deal with the functional facet of objects. Thread safety properties cannot be easily combined with functional properties since the reasoning mechanisms utilized by the functional facet of objects produce counter-intuitive results. Our contributions are identifying this issue and presenting a solution (see section 3.2) by decoupling the functional and thread safety facets of contracts. 


\section{SPECIFYING CONTRACTS IN THE PRESENCE OF CONCURRENCY}

The previous section explored the various problems encountered with using contracts to specify typical behavioural patterns of a concurrent object-oriented program.

This section presents a solution to the issue of interference and some of the instrumentation issues that are related to the semantics of contracts. It does not cover the problem of generating RAC code for each construct. Atomicity is never assumed to hold in any of this discussion. This complicates the argumentation but allows it to be more generic.

\subsection{Safepoints}

We start with a presentation of what a safepoint is followed by a discussion on how it can be used to solve some of the issues presented in section 2.4.

A safepoint is a point inside the method body at which it is safe to evaluate precondition or postcondition predicates together with object and class invariants. A method can have multiple safepoints. A precondition safepoint marks a point in which it is safe to compute the formulas of all preconditions and invariants, and the pre-state formulas of the selected postcondition. A postcondition safepoint marks a point in which it is safe to compute the formula of the postcondition selected at the time of the precondition safepoint and the invariants. Notice that this is the only postcondition for which thread safety is required to hold. No guarantees are made with respect to other postcondition formulas present in the method specification. A method execution path (from the first executable statement to the point in which it returns) can have only one precondition safepoint and only one postcondition safepoint. If no precondition safepoint is explicitly specified for a method, it defaults to the method pre-state. If a postcondition safepoint is not explicitly specified for an execution path, it defaults to the method post-state. 
We propose the addition of the requires_safepoint and ensures_safepoint labels to JML to demarcate precondition and postcondition safepoints, respectively. Listing 9 exemplifies the use of such constructs and how they avoid the issue of internal interference. At the precondition safepoint, all the objects referenced by both requires clauses and the contents of the lold statements in the ensures clauses are properly protected by locks. At the postcondition safepoint, the field head, present in the ensures clause of the second specification case, is properly protected by a lock. Since \result refers to the local variable $x$, it is also thread-safe at the postcondition safepoint. Finally, the object invariant can be safely evaluated both in the pre- and postcondition safepoints since it refers to the field head, which is properly locked in both places. Although not present in the specification for clarity purposes, access to field last is protected by (a lock on) head.

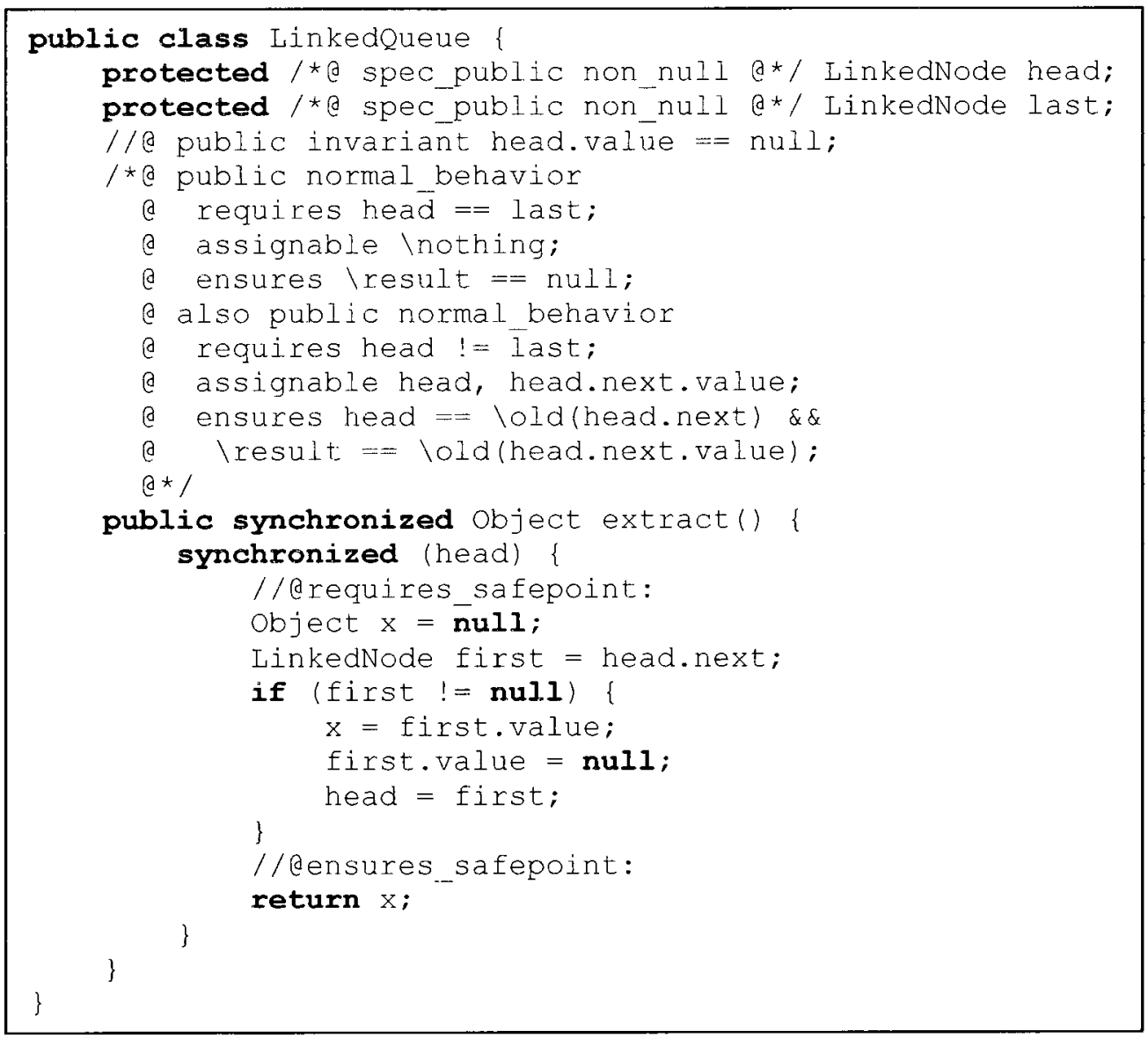

Listing 9: JML specification for the method extract () of the LinkedQueue class using safepoints to avoid internal interference. 
Having the precondition of a method, which is a predicate that specifies what needs to be satisfied prior to entering a method, evaluated inside such method is a counter-intuitive notion. A precondition, as initially presented by Meyer, can be thought as being evaluated just before entering the method or just after (i.e. before any statement of the method body is executed). These two views are equivalent because one knows that nothing significant to the evaluation of the precondition formula happens between these two states. The same idea applies to the precondition safepoint: nothing significant to the evaluation of the precondition predicate happens between the method's pre-state and the safepoint. That is, the precondition can be evaluated either in the pre-state or at the precondition safepoint yielding the same result. The only difference is that at the precondition safepoint the method is interference-free (due to the acquisition of some locks, in this example), thus allowing safe evaluation of expressions referring to shared data and ensuring proper correlation between the result of the precondition evaluation and the expected postcondition.

Analogously to preconditions, postconditions can be thought of being evaluated just before the method exits or just after it returns or throws an exception (i.e. in the poststate). Postcondition safepoints are another point to evaluate the postcondition predicate because nothing significant happens between such point and the methods' post-state. One must note that the postcondition safepoint is obviously located before the method returns. This brings up the issue of how to evaluate the postcondition expression since it refers to $\backslash$ result, the method's return value. The postcondition safepoint must be the label of the return or throw statement. The expressions in such statements must be side-effect free. This can be easily checked at compilation time by requiring side-effect free only operations and purity from invoked methods and constructors. In case the method does not return a value, the ensures_safepoint label can be placed at the end of a block or just before the method returns.

Two issues were purposefully left out of the above arguments: formulae involving method parameters and statements that are considered significant to the evaluation of the preconditions, postconditions and invariants. The former relates to thread-safety 
requirements, which are covered in the examples to follow. The latter is deferred to the section on formalization.

The example above shows how safepoints can be used to solve the issue of interference. Listing 10 shows how a precondition safepoint can be used to prevent external interference from causing problems in the presence of blocking behaviour (see 2.5). No extra safepoint is needed for the when clause, since it is evaluated at the commit point, which is already located in a thread-safe place. The issue this safepoint addresses is the case in which external interference causes the wrong when clause to be evaluated. The safepoint ensures that the values of ciosed observed by the formula and the method body are the same by moving the precondition evaluation point to inside the method body where it is not possible to change the value of closed between the precondition evaluation and its observation by the current thread. This example also shows the case in which no explicit postcondition safepoint is necessary. Since the postcondition formulas do not reference shared data they can be safely evaluated in the method's post-state.

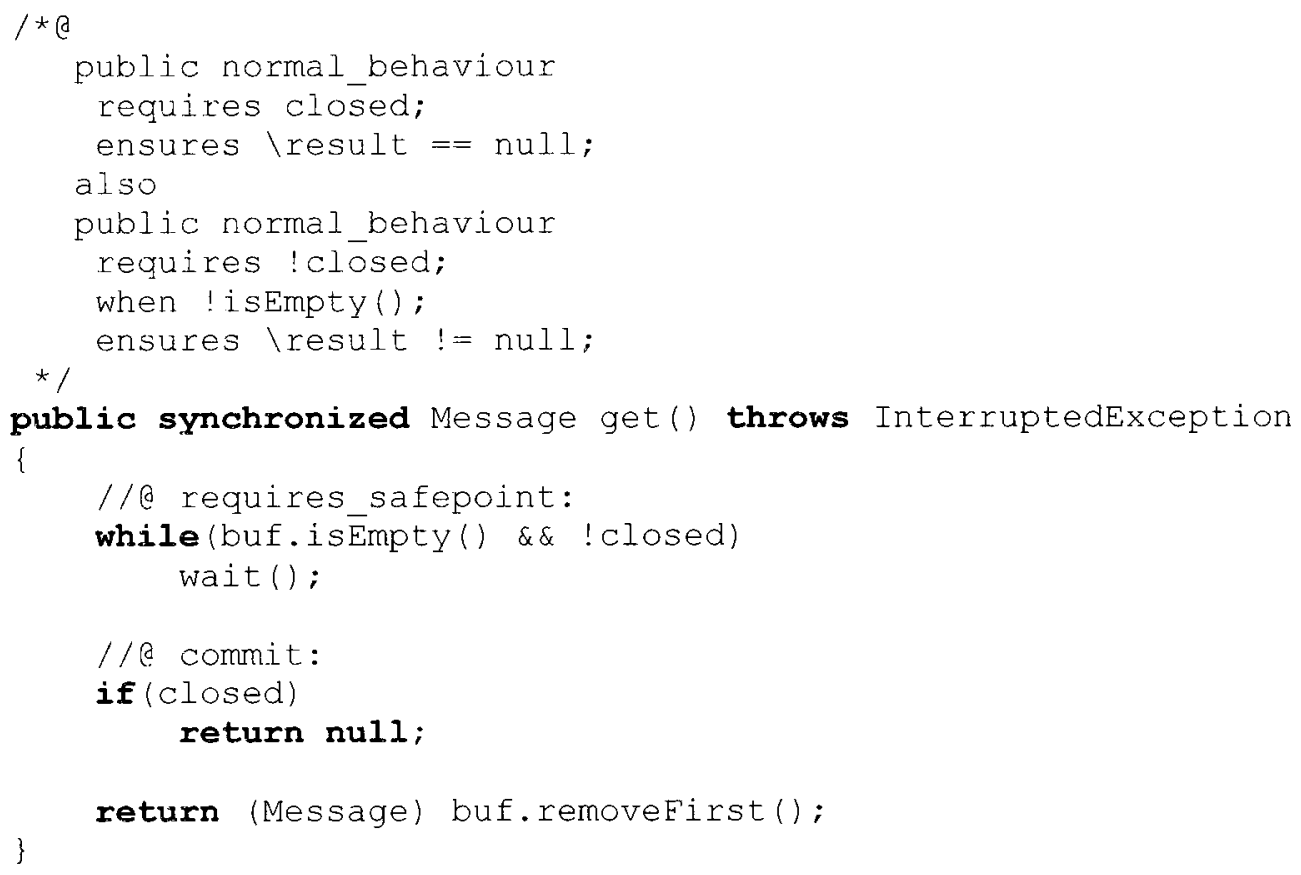

Listing 10: Using safepoints to prevent external interference in the presence of blocking behaviour. Excerpt of Listing 5 showing only the modified method. 
Safepoints can also be used to prevent interference in the presence of specification inheritance. Listing 11 shows how safepoints can be used to prevent external interference for method receive() of class Pipedchannel (see Listing 4). The specification for this method is inherited from the interface it implements (see Listing 3). A safepoint defines the place at which to evaluate the resulting specification (i.e. pre- or postcondition and invariants, including history constraints), which is the desugared specification taking into account all inherited specifications.

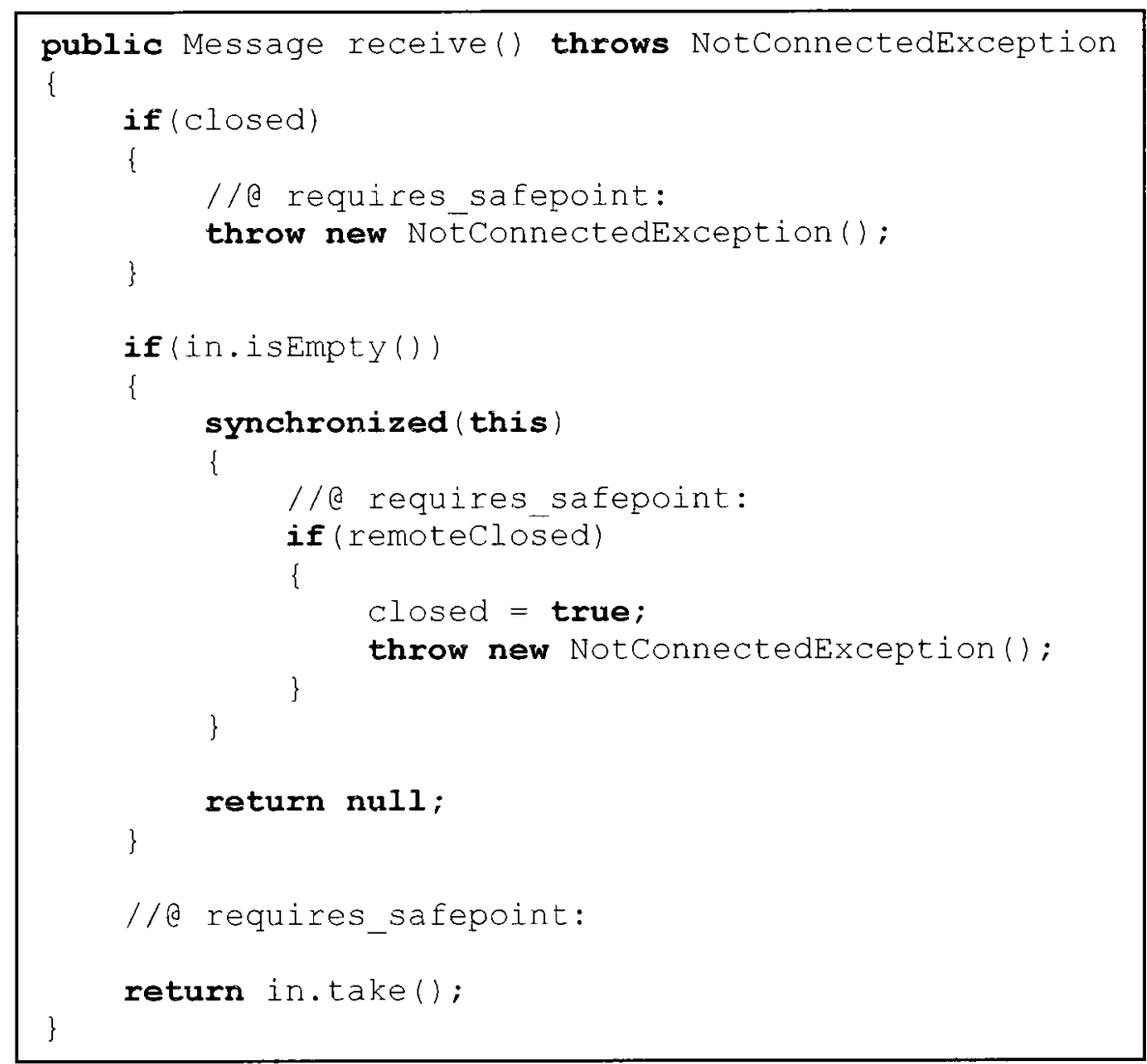

\section{Listing 11: Method receive() of class PipedChannel equipped with safepoints.}

Both specification cases reference model fields connected and remoteclosed. The values for these fields are obtained through the represents clauses defined in class Pipedchannel. The model field connected is realized through fields closed and remoteclosed of class Pipedchannel. The evaluation of connected needs to occur at a place where the concrete fields are protected from interference or after their values have already been obtained. An example of the former case is the second safepoint in Listing 
11. Access to remoteclosed is protected by a lock on this, which guarantees that the value observed by the precondition is consistent with the one observed by the method. An example of the latter case is the first safepoint in the same method. It is located after closed evaluates to true. By looking at the source code of the whole class (not shown here) one can see that once closed is set, it is never reset. Thus the safepoint is in a safe place to evaluate the precondition in case connected is false. Locking is not required in this case because Java guarantees that accesses and assignments to variables of type int and boolean are atomic. Otherwise, some locking would be required to guarantee atomic access to these fields.

There are two details about this example that need special attention. A closer look at the specification of receive() in Listing 3 shows a problem that is not addressed by the use of safepoints alone: the postcondition lresult $!=$ null $\Rightarrow=>$ nPending $==$ lold (nPending) - 1 cannot be always satisfied in case the left side of the implication is true. If another Message is put into the Pipe referenced by in after it returns but before the postcondition is evaluated in the method's post-state, the size() of in (which realizes npending) will not be one less of what it was at the precondition safepoint (the third one, in this case). Even if one were to add a postcondition safepoint right before the return statement, it would not be enough. This is due to the fact that the concurrency control happens inside the Pipe.get() method. Once it returns, thus releasing the lock on the Pipe, another thread is free to execute Pipe.put(). If one wanted to enforce the postcondition in question, one would have to acquire a monitor lock on in and include the pre- and postcondition safepoints together with the call to Pipe.get () in this synchronized block.

The other detail is regarding the history constraint. A history constraint is a type of invariant that specifies predicates regarding state transitions. In this example, it specifies that once connected becomes false it cannot become true anymore. The value of lold(connected) is computed together with the precondition safepoint. The value of connected is computed together with the postcondition safepoint, which in this example is in the method's post-state. Although not strictly thread-safe, it is safe to 
evaluate connected in the post-state due to the atomicity guarantees offered by the JVM. More sophisticated constraints might have more stringent locking requirements, which would affect the use of safepoints. It should be nevertheless clear that evaluating a constraint at a postcondition safepoint would entail the same result as in the post-state in an interference-free environment since nothing significant can happen between these two places.

Safepoints can also be applied to contracts exhibiting the combined behaviours of blocking behaviour and specification inheritance. Listing 12 shows such an example from section 2.5. By holding a lock on Pipe in and having the precondition safepoint located inside this monitor, one can prevent the issues of internal and external interference. The Pipe is locked while its number of elements is checked, the model field nPending is evaluated, and the preconditions are evaluated determining the postcondition to be evaluated at the postcondition safepoint and the when clause to be evaluated at the commit point. Notice that the super-class (i.e. PipedChannel) implementation of receive () is executed while holding a lock on in. This prevents the issues mentioned above regarding the satisfiability of the postcondition involving the number of messages in the channel for the nested call.

The use of safepoints does not solve the problem of breaking behavioural subtyping created by introducing blocking behaviour (see section 2.5 for more details). Even though interference is avoided, the precondition safepoint cannot prevent the Pipe from receiving messages while receive() is blocked. This can only be solved by relaxing the specification of Channel. receive() to offer weaker guarantees. 


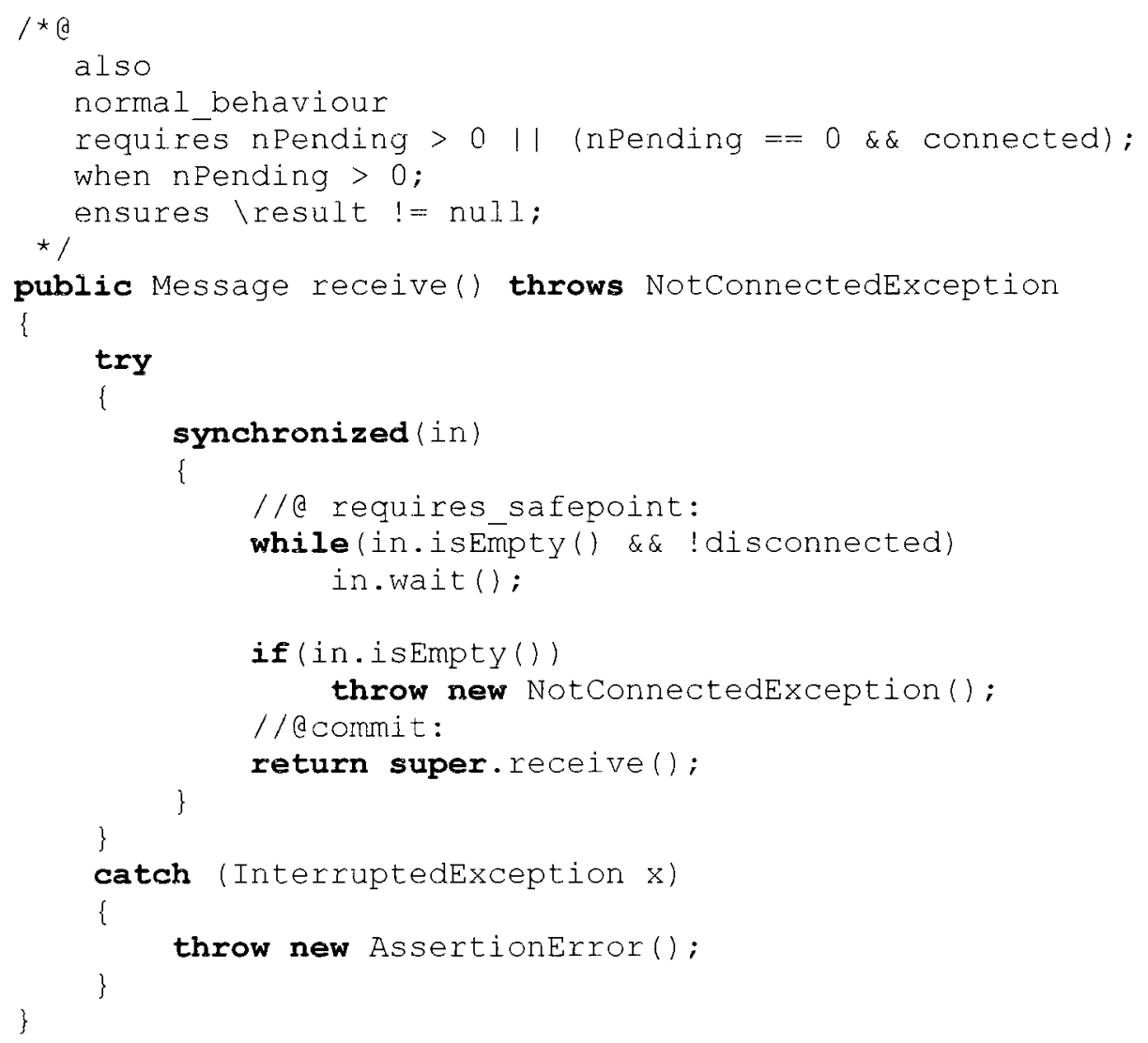

Listing 12: Method receive() of class BlockingChannel2 equipped with safepoints.

\subsubsection{Discussion}

Up to this point we only discussed cases in which contracts refer to the internal state of an object and the effective precondition is true. The problems safepoints solve are correlating preconditions to postconditions and guaranteeing thread-safe access to objects present in such formulas. In other words, they exist to demarcate a point in the code in which the value of the variables the pre- or postcondition predicate observes is the same as the one the method execution does. The idea is not to add extra concurrency control just for the sake of evaluating contracts since it would cause the instrumented code to execute differently, likely preventing harmful interleavings present in the original code from occurring, which could lead to undetected defects on the final (uninstrumented) program. Instead, the idea is to find the right place in the code to evaluate the contract 
following, to the extent possible, the informal reasoning of the designer, who was certainly not thinking that such predicates were all safely observable immediately before or after the method execution. For instance, when one specifies the behaviour of Linkedqueue.get () (see Listing 9) to "return null if the list is empty or the element at the head, otherwise" one is implicitly thinking "once the list can be safely manipulated, return null if it is empty or the element at the head, otherwise".

In this light, we consider safepoints not as part of the contract but as being part of the implementation ${ }^{11}$. Thus, correctly placing the safepoint is an implementation problem not a contract design problem. This approach is in line with the idea behind $\mathrm{DbC}$, namely that a contract specifies the observable behaviour of a method without getting into the details of its implementation. Placing the safepoint incorrectly will cause similar problems as not dealing with a valid case or generating an unexpected result for a valid input.

Of course, the placement of safepoints needs to follow the rules defined in the beginning of this section to guarantee the expected semantics of a contract, i.e. that preconditions and postconditions are evaluated only once for a method execution. These constraints are checked during compilation time by doing a simple flow control analysis to guarantee that only one precondition and one postcondition safepoint per execution path can be executed and that only unobservable statements exist between the beginning of a method and a precondition safepoint and between a postcondition safepoint and the end of a method. The technique to perform such a check is very similar to what is used to guarantee that a constant variable declared at the beginning of a method is only initialized once. Of course, such technique discards some valid cases since it is undecidable to determine if a safepoint is going to be executed more than once, in general.

The use of safepoints allows for the elimination of non-determinism in the specification of a concurrent method. Although a valid specification technique, the use of nondeterminism in a RAC scenario defeats the purpose since it would reduce the detail of the specification. The more detail present in a specification, the higher the likelihood of

\footnotetext{
1 Being part of the implementation, safepoints can only be defined in .java files. Other specification-only files accepted by JML (e.g. .jml) can only be used to specify contracts, as expected. Method body JML constructs such as assert and assume, too, can only be present in .java files.
} 
detecting a fault. Compared with existing techniques [22, 25], in which all variables referenced in specifications are required to be thread-safe, all the preconditions on the examples in this section would have to be reduced to true, i.e. no requirements, since all of them refer to non-thread-safe variables. We see value in being able to specify such properties.

\subsection{Specification of Thread-Safe Behaviour}

The specification of thread-safety properties can be divided in two types: locking requirements and thread-safety predicates. The former type deals with the specification of locks that must be held by the current thread to execute a particular method. The latter type deals with thread-safety of the objects used during the execution of a given method. As discussed in section 2.7, JML already provides constructs that allow the specification of these properties but these constructs present shortcomings we consider to be of fundamental importance in allowing their use in the presence of specification inheritance. In this section we present our solution to them emphasizing their use for RAC in conjunction with safepoints. Our solution considers all the challenges mentioned in section 2.7 .

The fundamental issues with the current approach to the specification of thread-safe behaviour are the predicate evaluation points and their meaning in the presence of specification inheritance. The root cause of these issues is the mixing of thread safe behaviour specification with functional specification.

Functional specifications deal with state transformations. They specify properties that must hold on the states preceding and following a method execution. Thread safety specifications deal with the properties that must hold to ensure that such state transformation occurs as specified in a concurrent environment. A program can, then, be seen as the combination of two facets: the functional and the concurrent. The functional facet is the one that deals with retrieving, processing and outputting data. The concurrent facet is the one that deals with the mechanisms to guarantee that access to such data by multiple threads is properly controlled. The functional facet specifies properties that depend on the client and the provider. The concurrent facet specifies properties that 
depend on the environment. The environment is everything else that happens outside of the current thread's control. The current thread affects the environment through the acquisition and release of locks. These concepts are so independent that one usually states informal requirements about thread safety independently of functional properties. Method specifications should reflect this independence.

The concurrent facet of a method specifies its thread-safety requirements and assurances. These predicates can take the form of the $\backslash$ thread_safe predicate as well as the form of locking requirements and assurances. Both styles serve the same purpose: to specify the conditions for the thread-safe execution of the target method. Depending on the situation, it is easier to specify directly that a particular lock must be acquired before a method execution than to state that all objects this method refers to are \thread_safe. There are situations in which locking properties are functional properties. For instance, in a lock server class, the act of acquiring a lock and returning it on a method that is supposed to do just that should be treated as part of the functional facet since it is used to fulfil the method's functionality. Specifications should reflect this dual purpose of locking properties.

We propose the addition of the concurrent_behaviour specification to a method specification. The syntax of this specification is given by the productions in

Figure 4 following the conventions in [13]. The production specification is extended with the addition of the optional production concurrent-spec. The other productions of specification are unchanged and therefore not reproduced in here. Refer to section A.6 of [13] for the complete production. 


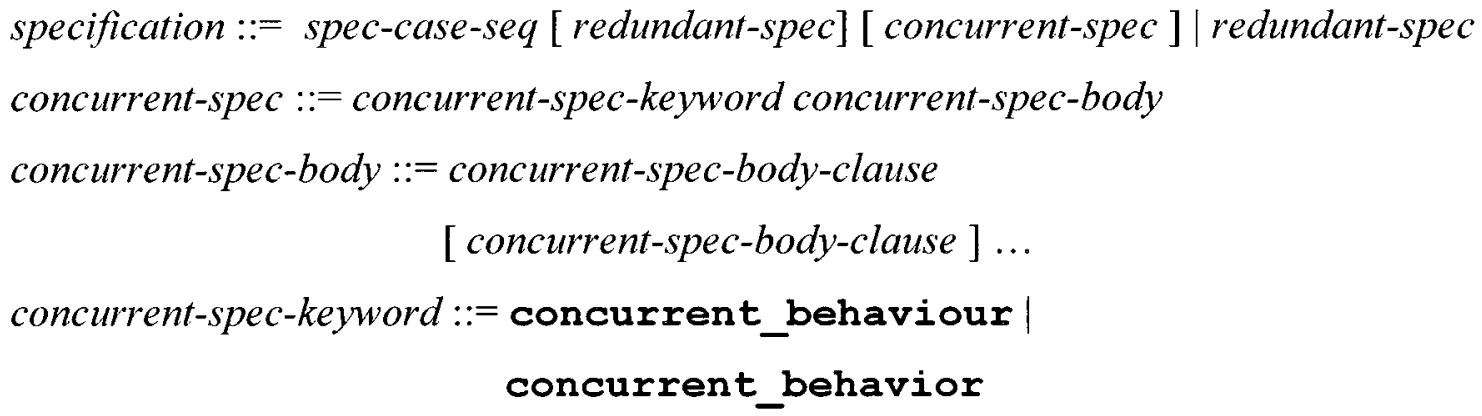

Figure 4: The modified specification production including the new concurrent-spec production

We propose the addition of the following clauses to the method specification:

- requires_locked, requires_unlocked, ensures_locked, ensures_unlocked: specifies that the set of lock objects provided are all held or not held by the current thread, respectively, in the method pre-state and post-state, respectively. null references are ignored.

- requires_thread_safe: specifies that all the objects provided satisfy the $\backslash$ thread_safe predicate in the method pre-state. null references are ignored.

- ensures_thread_safe: specifies that all the objects provided satisfy the $\backslash$ thread_safe predicate in the method post-state. null references are ignored.

We also make the locks clause part of the concurrent facet instead of the functional facet as originally proposed in [16]. This clause is intrinsically part of the concurrency control facet of programs since it is related to independence $[16,26]^{12}$. In this context, it is meaningless to allow for different specification cases to specify different locks clauses. This would mean that the client would have to either acquire the locks in the union of the set of locks specified in all specification cases or to selectively acquire the locks based on the specification case it is expecting to be satisfied. The former case trivially reduces to

\footnotetext{
${ }^{12}$ The execution of an independent statement or region is performed in an atomic fashion. Two adjacent independent regions can be thought of as a single independent region.
} 
specifying all the locks in the concurrent facet. The latter inductively reduces to the same situation for two adjacent methods to be treated as a single independent portion of code require that locks clauses from both to be satisfied. In such case, the client would have to acquire the locks from the union of the individual sets of locks specified on both methods' specifications. One should also note that the conditional operator ? : can be used to select the locks to be checked based on predicates evaluated at the pre-state thus presenting the same flexibility displayed by the use of multiple specification cases. This is important for situations in which the inductive reduction is not feasible due to design decisions (e.g. compliance to legacy interface).

The syntax of these clauses is given by the productions in Figure 5 following the conventions in [13]. The production spec-body-clause [13] ${ }^{13}$ is extended by having locking-body-clause as a new alternate production. This allows using the locking clauses in regular (functional) specification cases. We decided for using the keyword locks_releases instead of the proposed locks because requiring it to be a reserved keyword would have made most JML programs impractical since this would forbid referring to such identifier inside a JML annotation. One such instance would be the use of a model import directive related to the java.util.concurrent.locks package.

\footnotetext{
${ }^{13}$ The production spec-body-clause is defined in section A.6.
} 


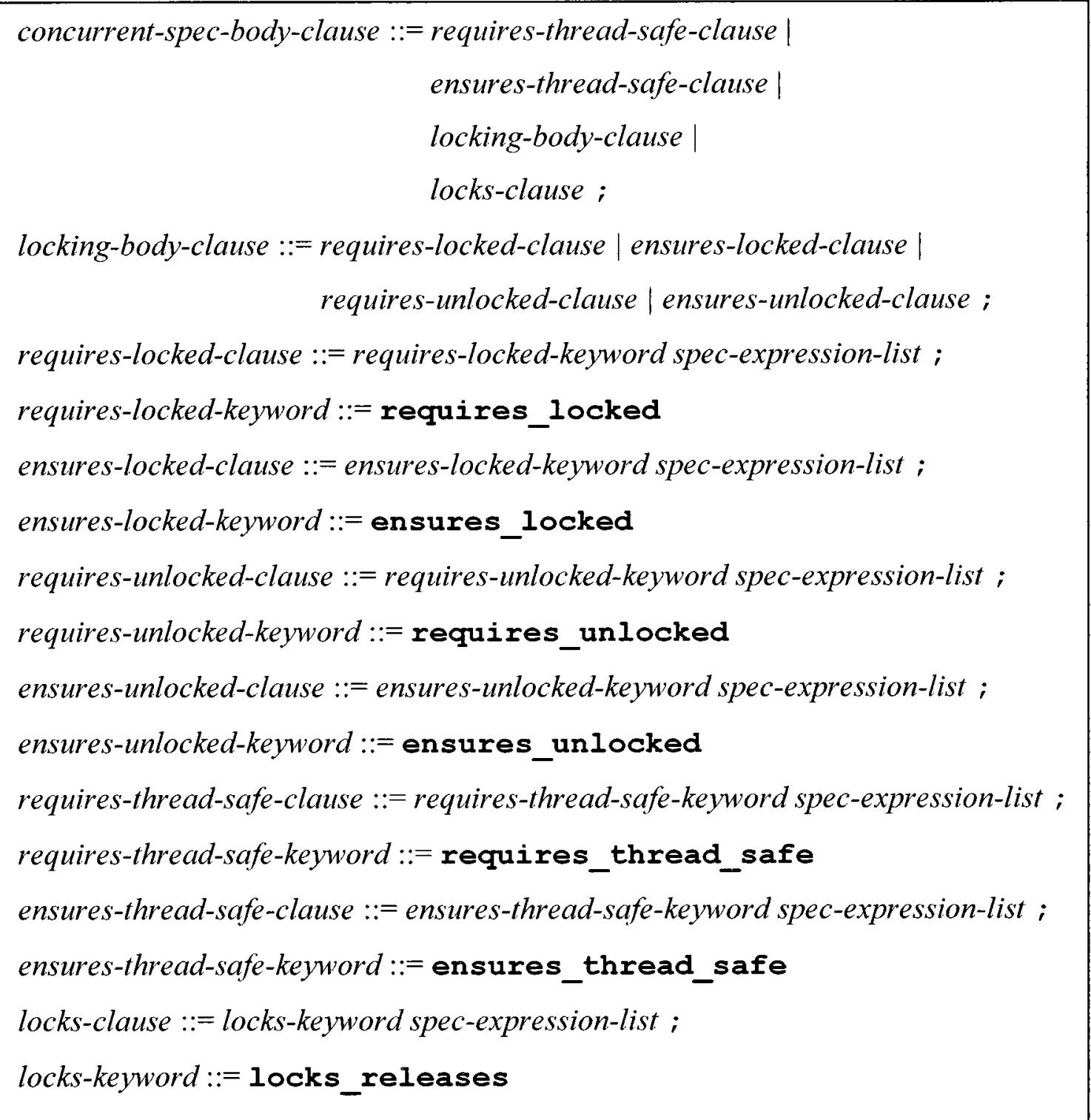

Figure 5: The productions specifying the newly introduced clauses.

Every expression in the spec-expression-list $[13]^{14}$ productions above must evaluate to an object reference. The semantics of a method specification that includes a concurrent_behaviour specification is the following:

\footnotetext{
${ }^{14}$ The production spec-expression-list is defined in section A.8.
} 
- requires_thread_safe, requires_locked and requires_unlocked must be satisfied in the method's pre-state. In case of inherited specifications, the effective specifications for these clauses must be satisfied. The inheritance semantics is discussed below. With respect to RAC, these clauses must be evaluated and satisfied prior to any requires clause (functional precondition) is evaluated.

- ensures_thread_safe, ensures_locked and ensures_unlocked must be satisfied in the method's post-state. In case of inherited specifications, the effective specifications for these clauses must be satisfied. With respect to RAC, these clauses must be satisfied for the implementation to be considered correct but they do not relate to the ensures clauses (functional postconditions), which can be evaluated prior to the post-state (i.e. a postcondition safepoint).

- locks_releases must be satisfied in the method's post-state. In case of inherited specifications, the effective specification of this clause must be satisfied.

If locking clauses are used in the functional facet of the specification, they are treated as regular predicates and subject to the standard specification inheritance and desugaring rules. The only difference is that they are not evaluated with their respective safepoints. requires_locked and requires_unlocked are always evaluated in the pre-state, and ensures_locked and ensures_unlocked are always evaluated in the post-state of a method execution.

All concurrent specification clauses default to \not_specified. The semantics of specification inheritance on the concurrent facet is exactly as for invariants. The effective specification of a particular clause is the set of reference objects resulting from the union of the argument set specified on the target object with the argument sets of its immediate supertypes. \not_specified is treated as the empty set.

Thread-safety specifications, like invariants, should only be strengthened by sub-types. At first, one might think that such properties should not be inherited at all since concurrency control is very particular to a type. Usually one would not see these properties as exposed functionality but as implementation details. However, it should be 
possible for a class to specify these properties for associated objects designed for extension. A typical example is a class in a messaging framework. The framework could define a Message interface and state locking and thread-safety requirements for implementers so that they can be handled without risk of deadlocks or race conditions. Another point for the proposed semantics is that there are cases in which interfaces are purposefully underspecified with respect to these properties to allow concrete implementations the freedom to choose their concurrency control strategy. This makes sense when there are two or more interfaces to be implemented and these must work in tandem. For instance, a particular message processor (implementing interface Processor) processes RPC messages (implementing interface Message). This processor can require stronger properties from the concrete message since it knows it will only process RPC messages.

The idea is that concurrent specification clauses specify environment invariants. Environment invariants specify properties that the environment (i.e. threads) must respect before and after a method execution. The only difference is that (functional) invariants are concerned about the object's state as seen by the current thread and environment invariants are concerned about the state of the environment as seen by the target object. These properties deal with the state of locks, which is the way threads interact. The target object observes the environment through such locks and determines if the environment is safe for the current thread to execute its method. The locks_releases clause may not seem to constrain the environment in the same manner as the other concurrent specification clauses. This is not so, however, since it communicates to the client the set of locks it should acquire for the independent execution of the associated method and is, thus, inherently related to its concurrency control given that independent regions of code are executed atomically and are, therefore, interference free by definition.

The declaration of method sendAndWait() of class BlockingChannel in Listing 13 demonstrates the use of thread-safety specification clauses. The requires_thread_safe clause specifies that the object aRequest refers to must be thread-safe in the method prestate. This clause must hold on the method pre-state. Similarly, ensures_thread_safe 
specifies that the object returned by the method execution must be thread-safe on the method's post-state. The use of locking clauses in the concurrent facet is analogous.

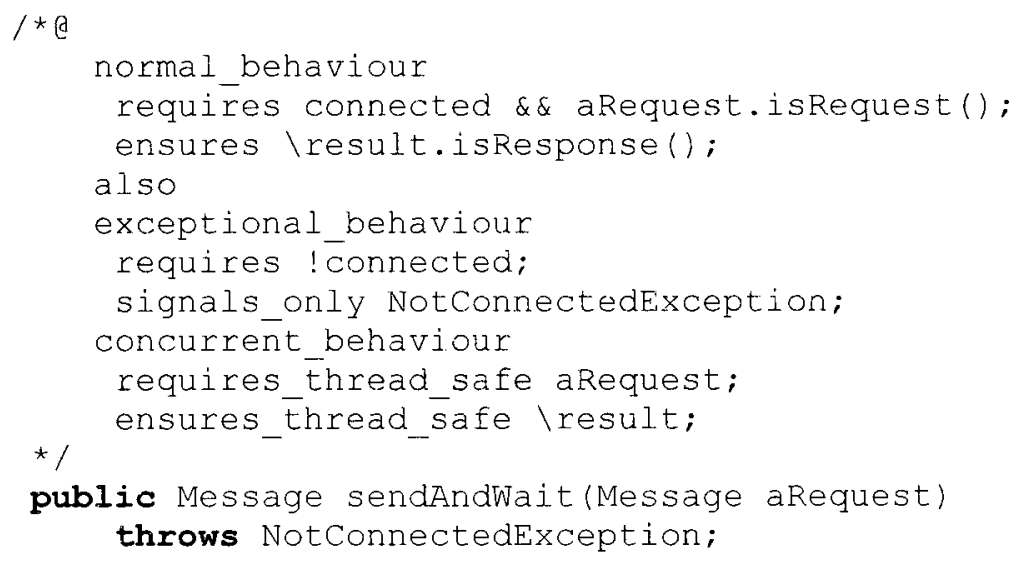

\section{Listing 13: Method declaration exemplifying the use of thread-safety specification clauses.}

The example on Listing 14 shows the use of locking clauses on the functional facet and the use of the locks_releases clause according to our proposal. This is a modification of the example on Listing 8 . This is just a syntactic example. The requires_unlocked clauses in both specification cases are evaluated in the method pre-state. They can be considered as being conjoined to the precondition of its associated specification case. The locks_releases clause on the concurrent facet specifies that the method must acquire and release a lock on this.

The semantics of these clauses allow for the specification of thread-safety and locking requirement properties in the presence of specification inheritance. It decouples concurrency related properties from functional properties giving concurrent contracts the intuitive (expected) meaning. We do not make any claims with respect to the modularity of the concurrent facet. This is outside the scope of this work. Our additions, however, have not disturbed modular reasoning on the functional facet since we did not change the way specification inheritance is implemented. 


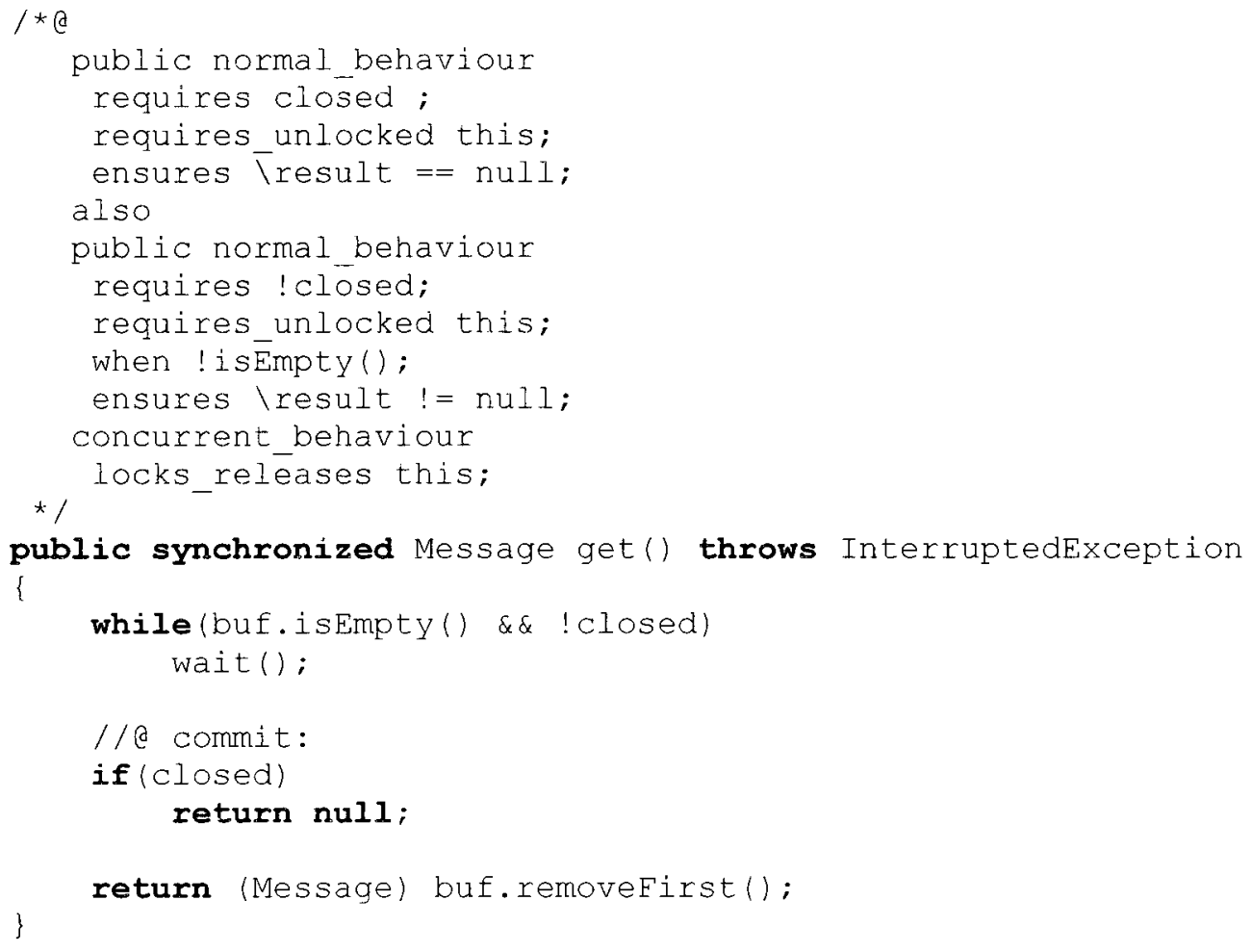

Listing 14: The contract for the get () method of the class Pipe using the new locking constructs.

\subsection{Thread Safety + Safepoints $=$ No Interference}

This section presents how one can combine the concepts of thread-safety with safepoints to solve the problem of interference. We describe this informally and then present a semiformal description of the semantics of a concurrent contract.

Safepoints alone cannot solve the issue of thread-safety when the effective precondition is not simply true, i.e. the client is required to establish a certain state prior to executing a method on the provider. Such a state can take the form of predicates on method arguments or predicates involving the internal state of the provider.

Listing 15 presents a simple contract in which the effective precondition involves predicates on method parameters. The effective precondition of method $\mathrm{m} 3()$ is $02 . \mathrm{p} 1$ (). Since it is the responsibility of the client to establish such predicate, it is also 
reasonable to expect that it provides conditions for the provider to safely observe it; otherwise it would be pointless to establish a state knowing it could asynchronously change before it could be observed. This is reflected by the use of the requires_thread_safe clause on all objects participating in the effective precondition. In this example, 02 is required to be thread-safe. Once such objects are thread-safe, the predicates on them can be checked at the safepoints since they will not change between the method pre-state and the precondition safepoint.

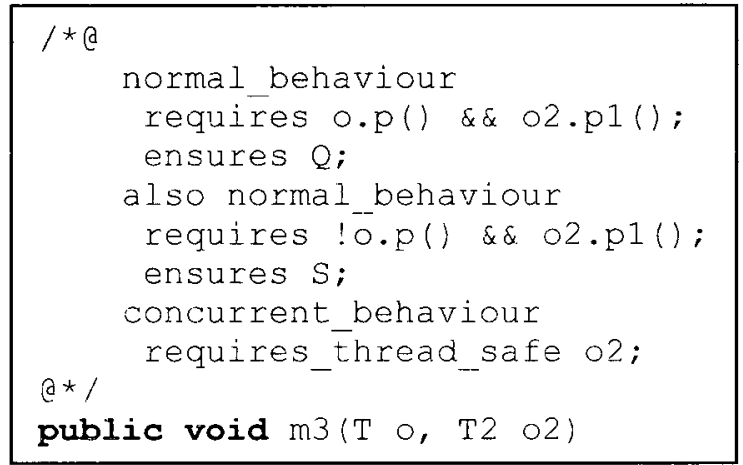

\section{Listing 15: Example of contract involving thread-safety requirements of method parameters. $Q$ and $S$ represent valid JML predicates.}

The requirements are similar with respect to postconditions. If such predicates involve any state that must be established by the provider and observed by the client, the associated objects must be flagged as thread-safe. If such objects are method parameters, then they should be covered by the ensures_thread_safe clause. If the return value is one of these objects, \result must be included in the ensures_thread_safe clause. A typical example of this case is a method that returns a collection and the postcondition specifies a membership predicate. Such collection must be thread-safe for such predicate to make sense.

The only meaningful case in which the internal state of the provider is present in the effective precondition in a concurrent environment is related to method invocation ordering requirements. This case is solved by using the locks clause on this or any specification accessible field designated as a lock protecting the explicit or implicit state machine implemented by such class in combination with safepoints to demarcate the safe 
place to check for the ordering predicate. Listing 16 exemplifies this situation. Method ev1 () can only be executed if the object is in state $\$ 1$ and it changes the state to $\$ 2$. The locks_releases clause tells the client that holding a lock on this guarantees the independent execution of the method. There are two basic forms of ensuring the correct use of such object. The client can be designed in such a way that it can never attempt an illegal transition (e.g. through a rendezvous mechanism between different threads) or competing threads can acquire a lock on the Statemachine object and call the event methods ev1 () and ev2() while in this monitor. The locks_releases clause guarantees independence to the client. 


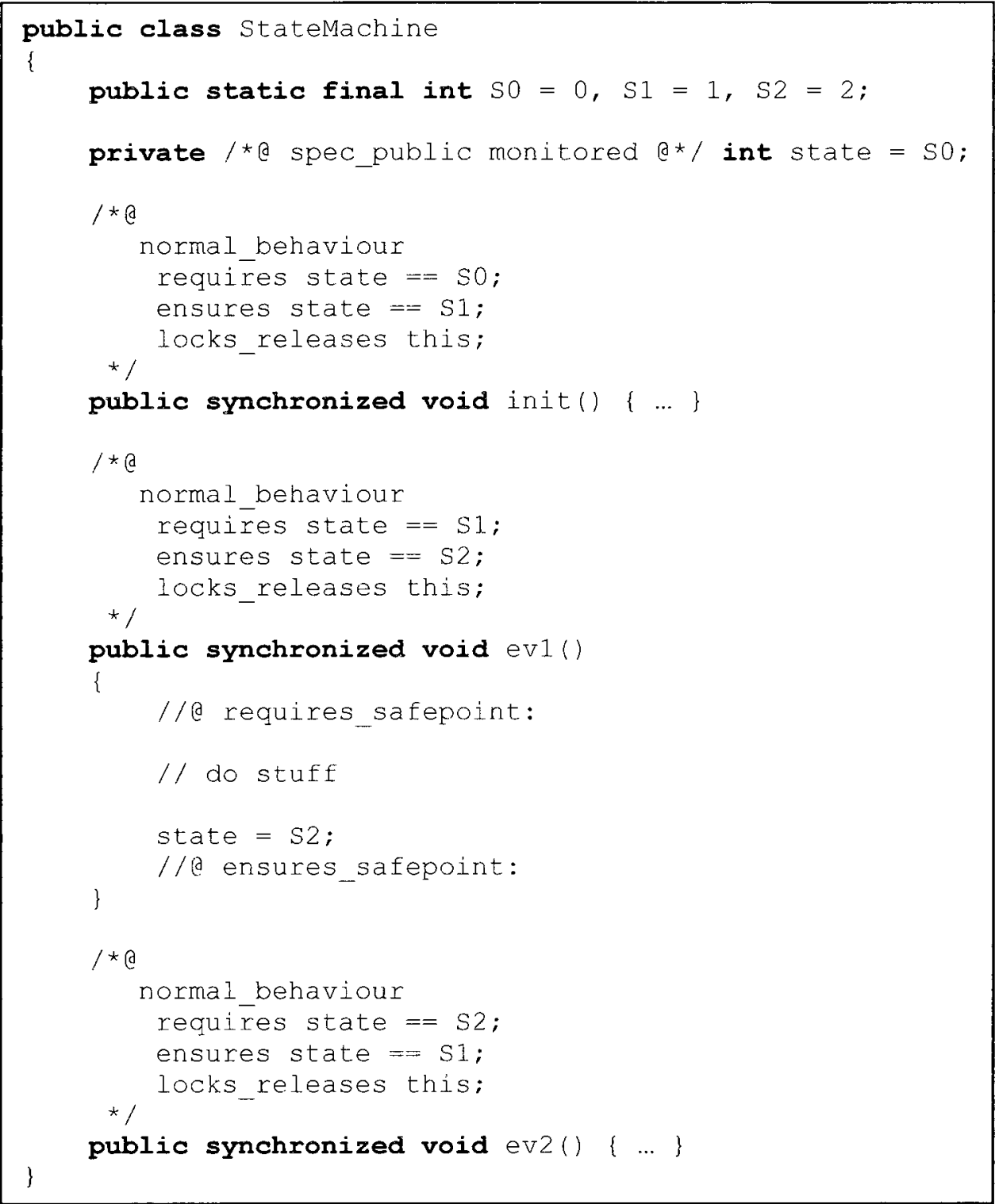

\section{Listing 16: Sample state machine designed for a concurrent environment. State}

changes are protected by synchronized methods.

The combination of thread-safety requirements for state that is established by the client to be observed by the provider and state that is established by the provider and observed by the client with safepoints, which demarcate a point for the thread-safe evaluation of expressions related to the internal state of the provider and objects passed as method parameters, guarantees freedom from interference. This has been presented informally through several examples in this section. We now present a semi-formal description of 
the semantics of concurrent contracts so one can understand their meaning and limitations.

\subsubsection{Informal Semantics of Concurrent Contracts}

Definition 1 (Unobservable Statement): The execution of a statement inside a method body is said to be unobservable if its effects do not change any state that can be observed by any user thread or it changes state local to the method body to which such statement belongs and it does not cause the method to terminate unless it does so by throwing a java. lang. Error exception. A user thread is the main thread or any thread created by the execution of a statement of a user thread. A state can be observed by a user thread if the object encapsulating it is reachable by such thread. A state is local to a method body if the object encapsulating it can be reached only via reference variables local to the method body or it is implemented by a primitive type variable local to the method body.

The idea behind an unobservable statement is that it does not produce any side-effects and does not interrupt the flow of control. The method terminating by throwing a java.lang.Error is not considered an observable event because this exception signals an internal error of the Java Virtual Machine and the method is, thus, released from any obligations (see section 9.6 .2 of [13]). This is considered an unrecoverable condition [11]. This definition is restricted to user threads because system threads like the garbage collection threads are not under program control and can observe any state at any point in time.

Lemma 1: The following statements are unobservable: assignment to local variables excluding arrays, if ... else, loops, try block, switch statement, break and continue. For the compound statements mentioned above, they are unobservable only if their substatements are unobservable. The evaluation of sideeffect free expressions abiding to the following constraints is also unobservable:

a. Array creation expressions are excluded;

b. Casts are excluded; 
c. Simple expressions must not refer to method parameters;

d. Field access expressions, method invocation expressions and array access expressions must refer to static fields or methods, or have a reference object equal to this, super, or a final field of this or super or of a static field that is non_null, or be a static constant expression (see section 15.28 of [27]).

Proof: All cases except the evaluation of side-effect free expressions follow immediately from definition 1. Since only side-effect free expressions are considered, only method termination must be proven impossible. The Java Language Specification describes the cases in which expression evaluations complete normally and those in which they complete abruptly by throwing an exception (see section 15.6 of [27]). The following cases must be guaranteed to not occur (the remaining cases cause a java. lang.Error to be thrown, which is unobservable by definition 1):

1. The dimension of an array in an array creation expression is less than zero;

2. A field access expression in which the object reference is nul1;

3. A method invocation expression in which the reference expression is null;

4. An array access expression in which the array reference expression is null;

5. A cast that is not permissible;

6. An integer division or integer remainder in which the right hand operator is zero;

7. An assignment to an array component of reference type is not compatible with the value to be assigned.

Constraint a eliminates cases 1 (trivially) and 7 (since only assignments to local variables are possible and constraint a prevents the creation of local arrays). Constraint $b$ eliminates case 5 trivially. Constraint $\mathrm{c}$ and $\mathrm{d}$ eliminate case 6 provided the programmer does not explicitly construct an expression that will cause a java.lang.ArithmeticException such as $3 / 0$ or $5 /$ (A.CONST - A.CONST) where $A$ is a class or interface and CONST is an integral constant. These constraints also eliminate cases 2, 3, and 4 provided the programmer does not explicitly construct expressions such as null. a( ) or $\mathrm{T} t=$ null; t.f(); . This is so because such expressions can only refer to objects and variables that 
are of known value (this, super and constant static expressions satisfy this trivially). Final fields of objects have their values set at construction time and a non_null modifier guarantees that they will be non-null at the end of construction and, therefore, for the remainder of the program execution.

The rationale is to eliminate cases the programmer cannot know the value of a variable that is non-local while allowing local expressions to compute derived values to be used in flow control statements. Pragmatically, even in the event that a programmer makes an unlikely mistake in a local expression, the RAC will intercept the exception and treat it similarly to a java. lang. Error, thus terminating the program.

Lemma 2: The following statements are unobservable: pure method or constructor calls that terminate normally, object allocation.

Proof: Pure methods are, by definitions, side-effect free. A pure method is not allowed to perform any assignments to any fields or to execute a non-pure method or constructor. A pure constructor can only assign to fields of the newly allocated object and perform pure method or constructor calls. Therefore, a pure method cannot perform any changes on the state of any object and thus, together with the restriction of normal termination, its execution is unobservable. A pure constructor is only establishing the state of an object that has been newly allocated which can only be observed by the local method body and is, thus, together with the restriction of normal termination, unobservable. Object allocation can only be observed by the internal JVM threads responsible for garbage collection and is, thus, unobservable. In case an object allocation fails, a java.lang.OutofMemoryError will be thrown. Since it is a subclass, of java. lang. Error, it is, by definition 1, unobservable.

Lemma 3: The following JML constructs are unobservable: annotation statements with the exception of the set statement (for ghost non-local variables), the assert statement, the assume statement, loop variant and invariant clauses.

Proof: By definition, the arguments of these constructs must be side-effect free expressions, including pure method calls or constructors. Therefore, the evaluation of 
their arguments is unobservable. The evaluation (in a RAC context) of these statements produces either:

1. No result, in the case of loop variants, loop invariants, assert, assume and annotations other than the set statement if the predicate is satisfied.

2. An assertion error being thrown in the case of loop variants, loop invariants, assert, assume, and annotations other than the set statement if the predicate is not satisfied.

3. An assignment to a local ghost variable.

For cases 1 and 3, it follows immediately from Definition 1. For case 2, assertion errors are subclasses of java.lang. Error [28] and it follows from Definition 1.

Lemma 4: The synchronized statement is unobservable in a deadlock free program.

Proof: Monitor locks are acquired and released by the use of synchronized statements. Monitor locks are reentrant, thus once a thread allocates a certain lock, it is guaranteed that it will succeed in acquiring it again (i.e. entering into a synchronized block). A thread succeeds in entering a synchronized block if the lock object has not been acquired by another thread. In a deadlock free program, every thread is guaranteed to progress, by definition. Therefore, every thread that enters a synchronized block is guaranteed to leave it. Thus, every thread that acquires a lock is guaranteed to release it. Thus, a user thread can enter and exit any number of synchronized blocks. Therefore, a user thread cannot observe any difference from executing a synchronized statement in which a lock is available and one in which it is not. Thus, it is unobservable.

Definition 2 (Safepoints): A precondition safepoint is a statement $\mathrm{S}$ identified by the requires_safepoint label in which the following holds:

1. A precondition safepoint is definitely not executed before $\mathrm{S}$

2. The preceding statements are marked as unobservable 
A postcondition safepoint is a statement $\mathrm{S}$ identified by the ensures_safepoint label in which the following holds:

1. A precondition safepoint is definitely executed before $\mathrm{S}$

2. $\mathrm{S}$ is either a return statement, a throw statement or, for a method with return type void, the empty statement at the end of a block after which the method is guaranteed to return normally.

3. If $\mathrm{S}$ is a return or a throw statement, $\mathrm{S}$ is composed only of unobservable expressions.

The notion of definite execution derives from the rules of definite assignment of Java (see chapter 16 of [27]). Every method can be thought of as declaring a local final variable named preSafe as its first statement. If the method does not contain any requires_safepoint label in its body then preSafe is assigned in the statement immediately following its declaration. Otherwise, preSafe is assigned a value at every requires_safepoint label for a statement $\mathrm{S}$. If such assignment is legal according to Java's assignment rules, then a precondition safepoint is definitely not executed before $\mathrm{S}$.

preSafe is read at every statement $\mathrm{S}$ identified by the label ensures_safepoint, at a return statement, at a throw statement and at the end of the method if it is of type void. If such accesses are legal according to Java's access rules, i.e. which only definitely assigned variables can be read then a precondition safepoint is definitely executed before $\mathrm{S}$.

A statement $\mathrm{S}$ is marked unobservable if:

1. It is an unobservable statement and its preceding statement is marked unobservable

2. It is an unobservable statement and it is the first statement in the method body 
Definition 3 (Concurrent Method): A (well-formed) concurrent method is a method that either does not require the use of safepoints or the following holds:

For each precondition safepoint:

1. All the locks necessary to evaluate all the precondition and class invariant predicates in a thread-safe manner are held by the current thread.

2. All the locks necessary to evaluate all the lold expressions present in all postcondition and history constraint predicates in a thread-safe manner are held by the current thread.

For each postcondition safepoint:

1. All the locks necessary to evaluate the postcondition associated with the specification case containing the satisfied precondition and class invariant predicates in a thread-safe manner are held by the current thread.

2. All the locks necessary to evaluate all the history constraint predicates in a thread-safe manner are held by the current thread.

A precondition safepoint is required if at least one precondition, invariant or $\backslash 01 d$ expression refers to an object for which access is not thread-safe or a consistent view of these objects is required for the correct evaluation of such predicates, and the required locks are only acquired inside the method body. A postcondition safepoint is required if the postcondition formula to be evaluated (as determined by the satisfied precondition) or any invariant refers to an object for which access is not thread-safe or a consistent view of these objects is required for the correct evaluation of such predicates, and the required locks are only held inside the method body.

It should be noted that the definition above implies thread-safety of all method arguments for which locks are not acquired inside the method body. Although there is no requirement to specify such arguments as thread-safe in the concurrent facet of the 
method specification, it is recommended as a matter of style to make it so. It enables static checkers to verify their validity and directs the RAC to include assertion checking code for them.

\section{Definition 4 (Effective precondition and Observable postcondition): The} effective precondition of a method is the predicate resulting from the minimization of the disjunction of all preconditions present in the method specification of the target class with all preconditions inherited from the method specification of its super-types.

The observable postcondition of a method is the predicate resulting from the minimization of the disjunction of all postconditions present in the method specification of the target class with all postconditions inherited from the method specification of its super-types.

The definition of effective precondition adheres to the notion of behavioural subtyping and, therefore, requires no further clarifications. The observable postcondition, however, presents a counter-intuitive notion that requires discussion. The idea behind it is that the predicate a method establishes is what is common between all postconditions as if all preconditions were satisfied. This notion is instrumental to determine thread-safety requirements, as will be seen below.

In a well-formed and realizable (implementable) method specification, specification cases have either mutually exclusive preconditions or have postconditions that evaluate consistently (for behavioural subtyping); otherwise the effective postcondition would be unsatisfiable. For mutually exclusive preconditions, the disjunction of the associated postconditions removes the terms that do not matter, since the method can, depending on the precondition, establish either a term or its complement. In the contract in Listing 15, the effective precondition is $02 . \mathrm{pl}($ ). For it to be an implementable contract, Q would have to be in the form $A \& \& B$ and $S, ! A \& \& \quad B$. This would lead to an observable postcondition equal to $B$. 
Theorem 1 (Interference Freedom): A concurrent method for which all objects referenced from the effective precondition and observable postcondition formula are thread-safe is interference-free.

Proof: For the case a concurrent method does not have any type and method specifications, it is trivially satisfied since no interference is possible, by definition. If safepoints are required, interference is prevented by the use of safepoints. This is so because precondition safepoints force the evaluation of preconditions to happen only at points in which access to all the referenced objects are protected by one or more locks. Objects referenced from specification cases that are not part of the effective precondition and the observable postcondition are part of the internal state of the target object or asynchronously established by the environment and, therefore, are protected by safepoints. Ojects referenced by the effective precondition or the observable postcondition are protected on the call site due to the thread-safety requirements and, therefore, are not subject to interference at safepoints. If the client guarantees sequential execution, internal interference is not possible. External interference is prevented for the same reason as in the previous case reduced to a case with no safepoints, i.e. preconditions and postconditions are evaluated in the method pre-state and post-state, respectively.

Theorem 2 (Equivalence of Concurrent and Sequential Contracts): A concurrent method for which all objects referenced from the effective precondition and observable postcondition formula are thread-safe is equivalent to a method with the same method and type specifications with safepoints and concurrent specifications removed, if executed in a sequential environment.

Proof: From definition 2, it is guaranteed that method preconditions are evaluated only once per method execution per construction. From the same reason applied to postconditions, it is guaranteed that the associated method postcondition is evaluated only once per method execution. Therefore; class invariants are evaluated twice per method execution: once with the precondition and once with the postcondition. History constraints are evaluated only at once together with the postcondition. Also, lold 
expressions are evaluated only once, together with the precondition

Also from definition 2, the restriction that there are only unobservable statements from the beginning (end) of a method and a pre- (post-) condition safepoint guarantees that the method itself does not modify the outcome of the pre- (post-) condition and invariants if they were evaluated at the method's pre- (post-) state (II). Theorem 1 guarantees that the evaluation of specification expression is consistent with the actual behaviour of the method

From I, II and III above, a concurrent contract for a concurrent method presents the same behaviour as the same contract without the concurrent features if applied to a method in a purely sequential environment.

To summarize this section, a concurrent contract can be applied to the same method in a sequential environment and the two executions are guaranteed to evaluate to the same results. More loosely: a concurrent contract presents the same semantics of its sequential version. Notice, however, that it is not guaranteed that every sequential contract can be applied to a concurrent environment. Examples in previous sections (see section 2.5 and Listing 6) presented situations in which some predicates could not be guaranteed to hold even in an interference-free environment without changing the method implementation.

\subsection{Lock Acquisition Order Specification}

This section presents our solution to the problem of specifying lock acquisition ordering policies for concurrent classes. In section 2.6 we pointed out several shortcomings of the current practice, namely the possibility of unexpected behaviour, the unnecessary flexibility causing unwanted complexity for the verification task, and the asymmetry of the construct that made it difficult to be considered as a class invariant.

We propose the addition of a type specification clause $[13]^{15}$ called lock_order to JML. The syntax of this clause is given by the productions in Figure 6, following the conventions in [13].

\footnotetext{
${ }^{15}$ A type specification clause is a production of jml-declaration (see section A.5). All modifiers are applicable to this clause as well.
} 


$$
\begin{aligned}
& \text { lock-order-clause }::=\text { lock-order-keyword lock-order-expression-list ; } \\
& \text { lock-order-expression-list }::=\text { lock-order-expression }[\text {, lock-order-expression }] \ldots \\
& \text { lock-order-expression }::=\text { spec-expression l-order-op spec-expression } \\
& \text { l-order-op }::=<\mid<= \\
& \text { lock-order-keyword }::=\text { lock_order }
\end{aligned}
$$

Figure 6: The productions specifying the lock order clause.

The semantics of the ordering operator is the following. Let 11 and 12 be instances of java. lang. Object. The expressions $11<12$ and $11<=12$ evaluate, respectively:

1. true and true, if the current thread acquired lock 11 before 12 or if it acquired 11 but not 12 .

2. false and false, if the current thread acquired lock 12 before 11 or if it acquired 12 but not 11 .

3. false and true, if the current thread did not acquire neither 11 nor 12.

The type of the two spec-expressions must be either java.lang.object, or org.jmlspecs.models.JMLCollection. In the latter case, the ordering operator is applied to each element of the collection and the lock-order-expression evaluates to true if, and only if, it evaluates to true for each such element. In case both spec-expressions are of type org.jmlspecs.models. JMLCollection the ordering operator is applied to every pair of elements according to the Cartesian product of the two sets. For instance, the lock-order-expression $a<\{b, c\}$, where $\{b, c\}$ represents a org.jmlspecs.models. JMLCollection with elements $b$ and $c$, is equivalent to two lock-order-expressions $\mathrm{a}<\mathrm{b}$ and $\mathrm{a}<\mathrm{c}$. Analogously, $\{\mathrm{a}, \mathrm{b}\}<\{\mathrm{c}, \mathrm{d}\}$ is equivalent to: $a<c, a<d, b<c, b<d$.

The semantics of the lock-order-clause is that each lock-order-expression must hold for every state it is in effect in the context of the current thread. A lock-order-clause is in 
effect for a given state if such state is in the activation record of a method belonging to the type declaring such a clause or one of its subtypes.

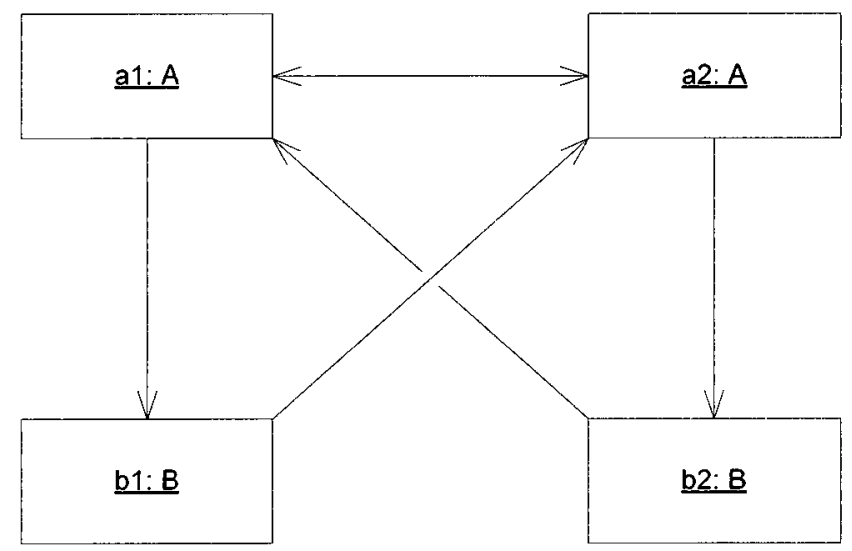

Figure 7: Object interconnection diagram to exemplify the semantics of the lockorder-clause.

These concepts are better understood through an example. Let class A define lock order clause La and class B, Lb. Also, let objects a 1 and a2 be instances of A and b1 and b2, of $\mathrm{B}$, and interconnected as depicted in Figure 7. Let thread $\mathrm{T}$ call method test () to perform such initialization and then al.m1 () such that the execution shown in Figure 8 occurs (the methods in question can return at the several '. . . ' for different reasons that are not relevant to this example) as in Listing 17. Let Lal and La2 denote La bound to objects a 1 and $a 2$, respectively. Let $L b 1$ and $L b 2$ denote $L b$ bound to objects $b 1$ and $b 2$, respectively. 


\begin{tabular}{|c|c|}
\hline 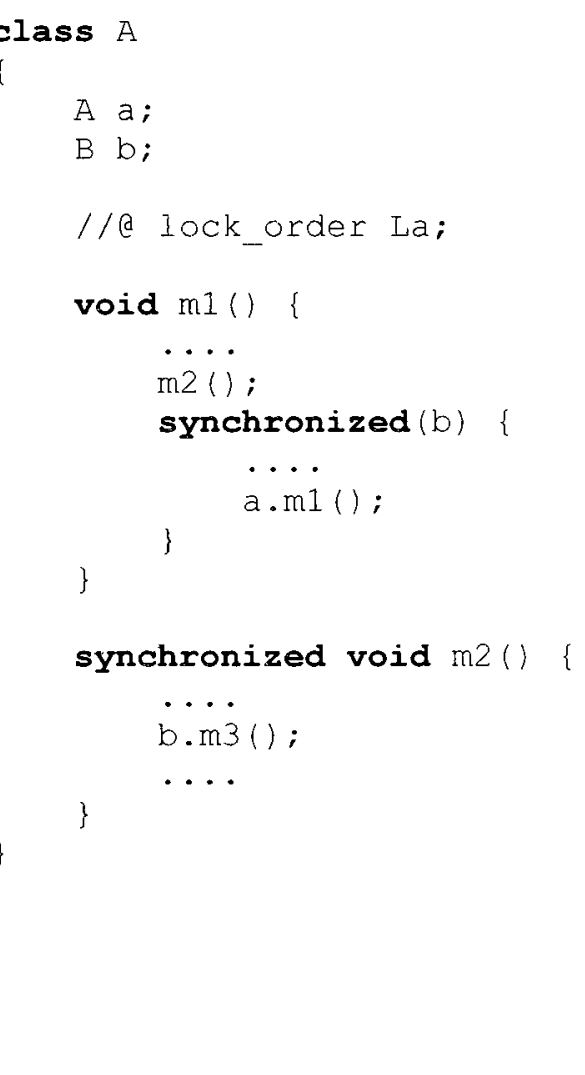 & 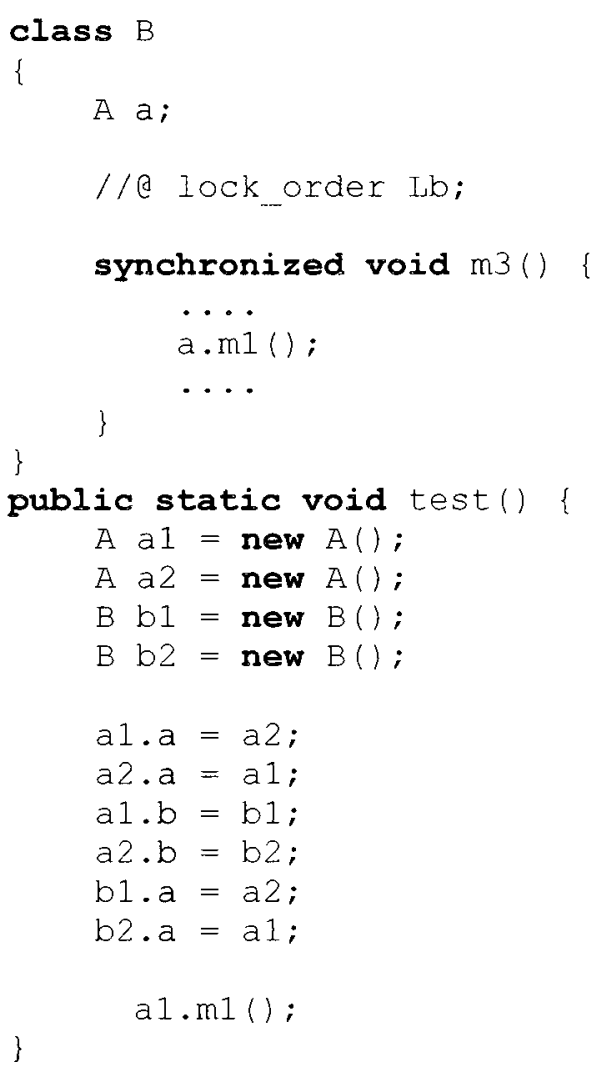 \\
\hline
\end{tabular}

Listing 17: Code fragments exemplifying the use of the lock-order-clause.

When thread $\mathrm{T}$ reaches point 1 , the only lock order clause in effect is La 1 . La 1 is still the only one in effect at point 2 . When it gets to point 3, La1 and $\mathrm{Lb} 1$ are in effect, since $\mathrm{T}$ entered $m 3$ () of $b 1$ while still inside $\mathrm{m}_{2}$ () of al. At points 4 and 5 we have Lal, Lbl and La2 in effect. At point 6, we have only La1 since $\mathrm{m} 3$ () of b1 has already completed its execution.

The question, now, is what it means for a lock order clause to be in effect. It means that it is evaluated at every attempt of thread $\mathrm{T}$ in acquiring a lock. 


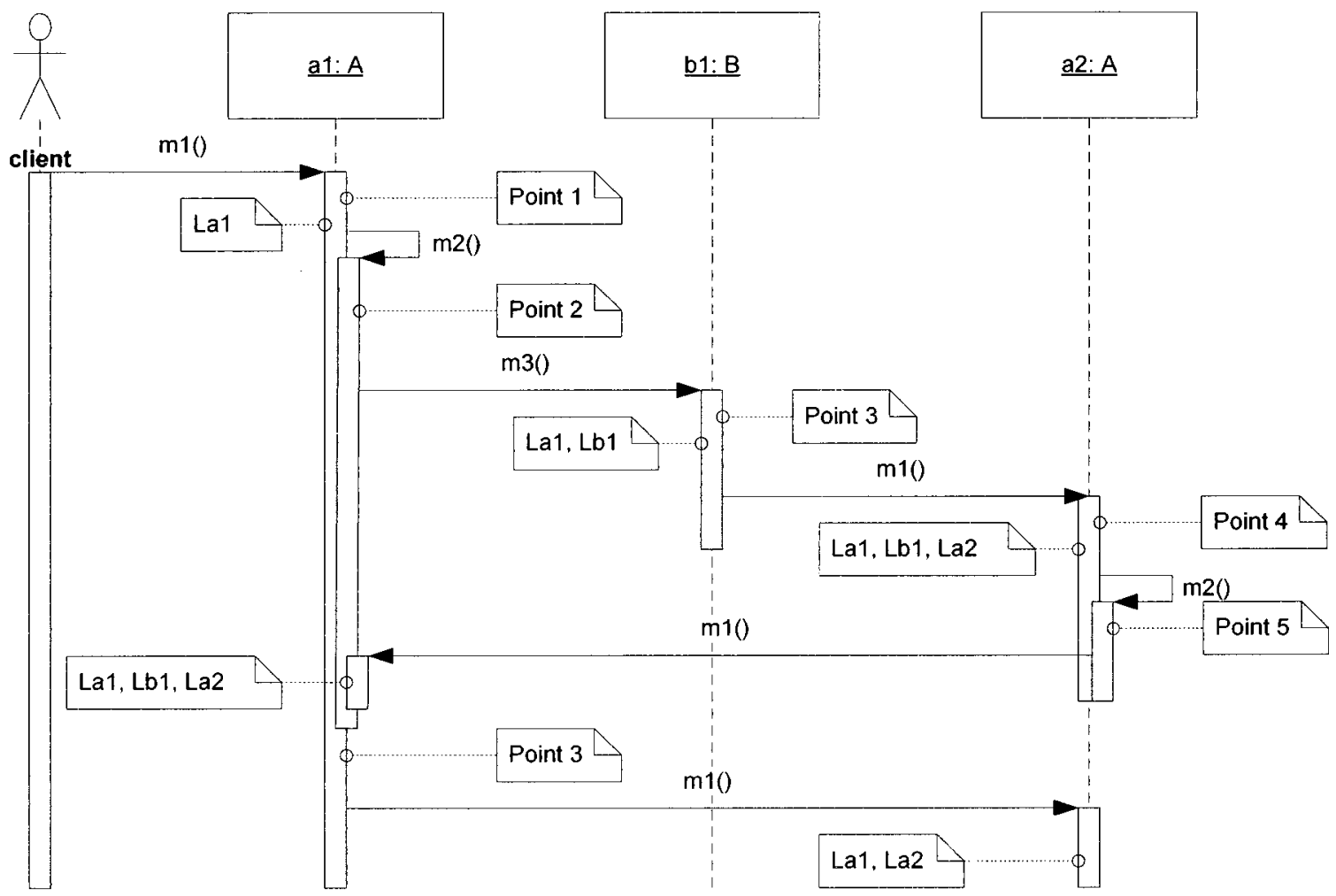

Figure 8: Sequence diagram depicting an execution instance of method a1 . $\mathrm{m} 1$ () for the running example. The notes attached to activations denote points in the execution referenced in the text and the lock order clauses in effect for such activation.

Continuing on the example, let La be this $<a$, this $<b, b<=a$ and Lb be this < a. At point 1, although La1 is in effect, no locks have been acquired, so La1 has not yet been evaluated. Lal will be evaluated for the first time at point 2 , just before the lock a 1 is acquired (as part of the synchronized method a $1 . \mathrm{m} 2(1)$ ). It evaluates to true since this is about to be acquired and the lockset for thread $\mathrm{T}$ does not contain a 2 or $\mathrm{b} 1$, the objects referenced by members $\mathrm{a}$ and $\mathrm{b}$ of $\mathrm{a} 1$. To reach point $3, \mathrm{~T}$ must acquire a lock on $\mathrm{b} 1$, since $m 3()$ is synchronized. At that point, $L a 1$ and $L b 1$ are in effect, so both are evaluated. Lal still holds since al (this in the context of a1) was acquired prior to b1 (the one being acquired by $\mathrm{m} 3())$ ). $\mathrm{Lb} 1$ is also satisfied since $\mathrm{T}$ is attempting to get a lock on $\mathrm{b} 1$ and it still does not hold a lock on a2, to which b1. a is bound. Point 4 does not add any evaluations, since no locks are acquired on the path from point 3 . Point 5 , however, is 
quite interesting, since $\mathrm{T}$ is attempting to lock $\mathrm{a} 2$ on $\mathrm{a} 2 . \mathrm{m} 2()$. La 1 is still satisfied since $a 1$ has been acquired prior to $b 1, a 2$ is being acquired at this point (the this $<a$ of La, with this bound to $\mathrm{a} 1$, and $\mathrm{a} 2$ to $\mathrm{a}$ ) and $\mathrm{b} 1$ was acquired prior to $\mathrm{a} 2$ (the $\mathrm{b}<=\mathrm{a}$ part). Lb1 is still satisfied for the same reason as before. La2, however, is NOT, since a1 was acquired prior to a2 (the this < a part of La2 with this bound to a2, and a to a1). Note that if a. $\mathrm{ml}$ () had reassigned the value of member a to point to another object, say a3, La2 would have been evaluated with a bound to a 3 and would, then, be satisfied.

It is quite obvious that this is an example of a deadlock-prone system once another thread is introduced, since the threads would attempt to acquire the same locks in a different order if they executed $\mathrm{m} 1$ () for $\mathrm{a} 1$ and $\mathrm{a} 2$ concurrently. The evaluation strategy described above for lock order clauses resembles the idea of axioms for ESC/Java with the exception that it is not prone to the erroneous deadlock warning message mentioned in [19], as discussed in section 2.6. This is due to the fact that the lock order clause is reevaluated every time a lock acquisition is attempted, solving the inconsistency problems mentioned above. One fine point that should be noted is that there is no need to use the universal quantifier to apply the locking predicate to all instances of a class. The fact that all lock order clauses are evaluated at every lock acquisition point for all instances being manipulated by a thread generates the same effect.

The example above shows how a lock order clause can be used to specify lock acquisition ordering policies and their effect. Specifically, the evaluation at point 5 of La2 illustrates a case in which a lock order clause is violated. One should note that this violation occurs due to the cyclic nature of the example. The interesting point here is that it is not prone to inconsistencies since every acquisition is seen as a potential cause for its violation, so it is re-evaluated in its (attempted) new condition. In summary, the lock order clause allows the local specification of locking policies with global effects. Its simplicity enables sound static and dynamic (RAC) verification of such policies, which is described in detail in section 4.5. 


\section{THE CONCURRENT RAC}

This chapter describes the instrumentation techniques used to implement the checking of the concurrency related clauses introduced in chapter 3 as well as existing constructs not previously supported in jmlc. It starts by describing the functioning of jmlc and the runtime, the collection of support classes needed during the execution of the instrumented code. There are two main tasks for enabling the runtime to support checking assertions of concurrent programs, namely adapting the bookkeeping required for supporting assertion checking in recursive calls and calling Java methods in contracts, and extending its functionality to keep track of locks. These are described in section 4.2. The instrumentation of safepoints and wait conditions is described in section 4.3. Threadsafety instrumentation and support is described in section 4.4. Lock order code generation and support is described last.

The process of RAC code generation can be formally described as a translation function that maps JML statements into Java program statements. Such functions are defined for most JML constructs in Cheon's work [28]. In this chapter we decided for an example driven approach to simplify the exposure and to focus on the techniques necessary to support concurrency in contrast to Cheon's goal of addressing JML semantic ambiguities and mismatches with Java's. This approach does not reduce the generality of the results since we introduced only a few translation functions and modified the existing ones. Moreover, the new and affected rules are restricted to type and method specifications, and thus there are only three pieces of information to be abstracted from the examples to generalized them into translation functions: the name of the type being translated, the name of the method being translated and the name of the lock being acquired or released.

\subsection{Runtime Assertion Checking Code Generation}

The JML compiler translates method specifications, invariants and constraints into assertion methods, which are called to check the respective assertions at appropriate points in the code. This facilitates specification inheritance and modularity since one can 
simply call an assertion method in its supertype to accomplish specification inheritance. Assertion methods become members of the class to which the specifications belong. In case of specifications belonging to interfaces, a separate class, called a surrogate class, is created to hold the assertion methods since interface methods in Java must be abstract. Calls to assertion methods in the surrogate class as well as superclasses are dynamically dispatched from subtypes. This allows for the separate compilation of source files, i.e. supertypes may or may not be compiled with assertion checking enabled.

Method specifications are checked via a wrapper approach. The original method is renamed to a private method and a new wrapper method is created with the same name and signature as the original method. As a result, all client calls are handled by the wrapper method. Invariant and precondition assertion methods are called prior to calling the original method. Invariant and postcondition assertion methods are called after calling the original method. This has the effect, from a client's perspective, of checking invariants and preconditions in the method's pre-state and checking invariants and postconditions in the method's post-state.

Listing 18 shows a simplified version of the wrapper method for the method extract () of class LinkedQueue shown in Listing 1, page 11. Line 7 shows the call to the original method. Lines 1 to 3 show the call to check for the object invariant. Line 1 signals to the runtime that the system is entering into the checking state. This effectively disables the execution of assertion code that would otherwise be executed as part of the evaluation of expressions contained in predicates specified in the clause being checked, since expressions in assertion code can call Java methods. The check in line 0 would succeed for a method called as part of a contract evaluation. Line 2 calls the private method to check the invariant predicate. Assertion methods throw an instance of JMLAssertionError (a subtype of java.lang.Error) to signal a violation of the predicate being checked. Line 3 signals to the runtime that the system is leaving the checking state. This pattern repeats itself in the wrapper method to check for preconditions (lines 4-6), exceptional postcondition (lines 8-10), normal postcondition (lines 14-16), and object invariant in the post-state (lines 11-13 and 17-19). Note that the exceptional postcondition may refer to the exception thrown, thus the corresponding 
assertion method takes the exception object as an argument (line 9). Similarly, the normal postcondition (line 15) takes the method's return value as an argument, which becomes the value of $\backslash$ result in normal postcondition predicates.

The calls to the runtime described above (i.e. through static methods of class JMLChecker) manipulate what we call the global checker state. The global checker state contains information that affect the overall behaviour of assertion checking, such as if the RAC is in checking state (as above), and the cache of loaded classes indexed by type name (used for efficient dynamic method dispatch to surrogate classes and supertypes). 


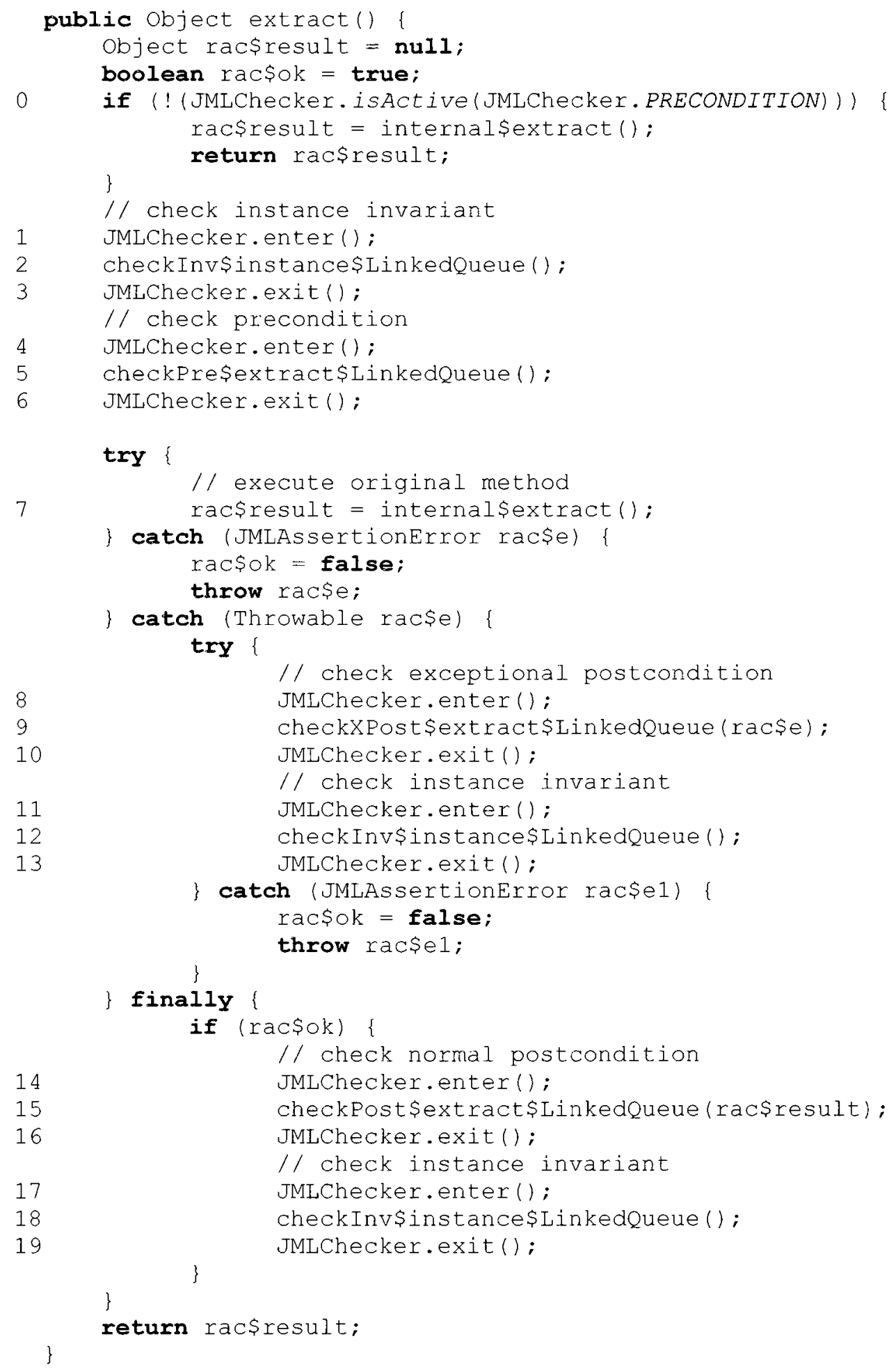

\section{Listing 18: Simplified wrapper method for method extract () of class Linkedqueue.}


Invariant assertion methods simply evaluate the predicates on the target object and conjoin them with the result of the evaluation of any inherited predicate. Precondition assertion methods (Listing 19), however, are required to store the result of the evaluation of such predicates so it can be retrieved by postcondition assertion methods to enable the evaluation of the predicates in the same specification case (see section 2.1). Line 3 records the result of the precondition for the first specification case on object fields (lines 1-4, Listing 1, page 11). Line 4 does the same for the second specification case. Line 5 stores these results into a method stack. The result of old expressions (lines 8 and 9, Listing 1, page 11) computed in lines 1 and 2 are also stored in this stack. The function of this stack is to support method assertions in recursive calls. The postcondition assertion method (Listing 20) pops the stack (line 1) and, based on the recorded outcome of the precondition (lines 2 and 4), it evaluates the appropriate postcondition (lines 3 and 5). The old expressions are replaced by the result of their evaluation in the pre-state. The same mechanism applies in cases of specification inheritance. The difference is that the evaluation of the inherited cases is performed on assertion methods in supertypes trough dynamic invocation.

The generated method stack and fields to store the precondition evaluations as well as the old expressions are amongst what we call the local checker state. The local checker state is stored in each object (and class to support checking of static methods) being checked as opposed to the global checker state, which is stored as a collection of static fields of the JMLChecker class. The local checker state also stores information about surrogate classes to be used when executing assertion methods inherited from interfaces. 


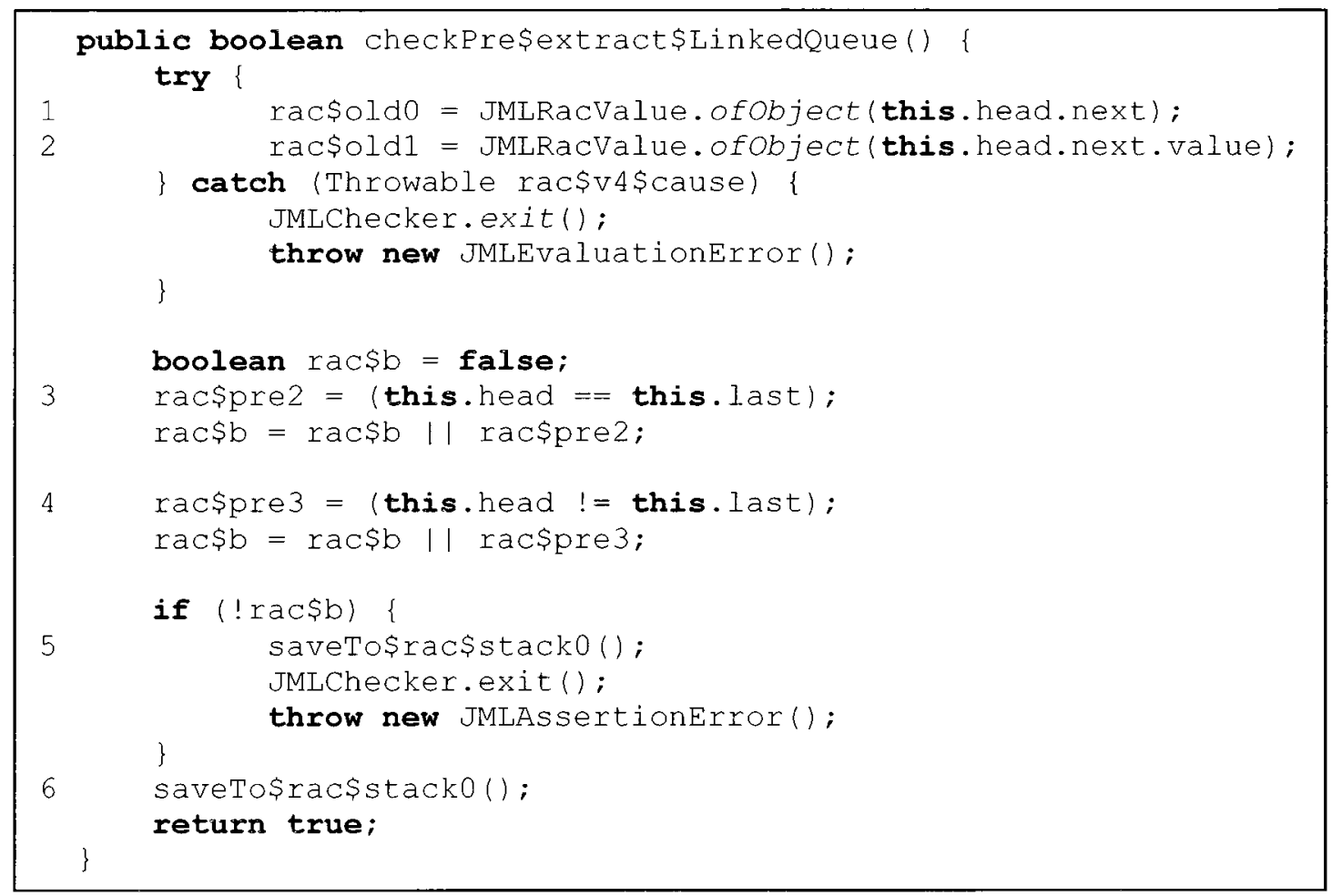

Listing 19: Simplified precondition assertion method for method extract () of class LinkedQueue.

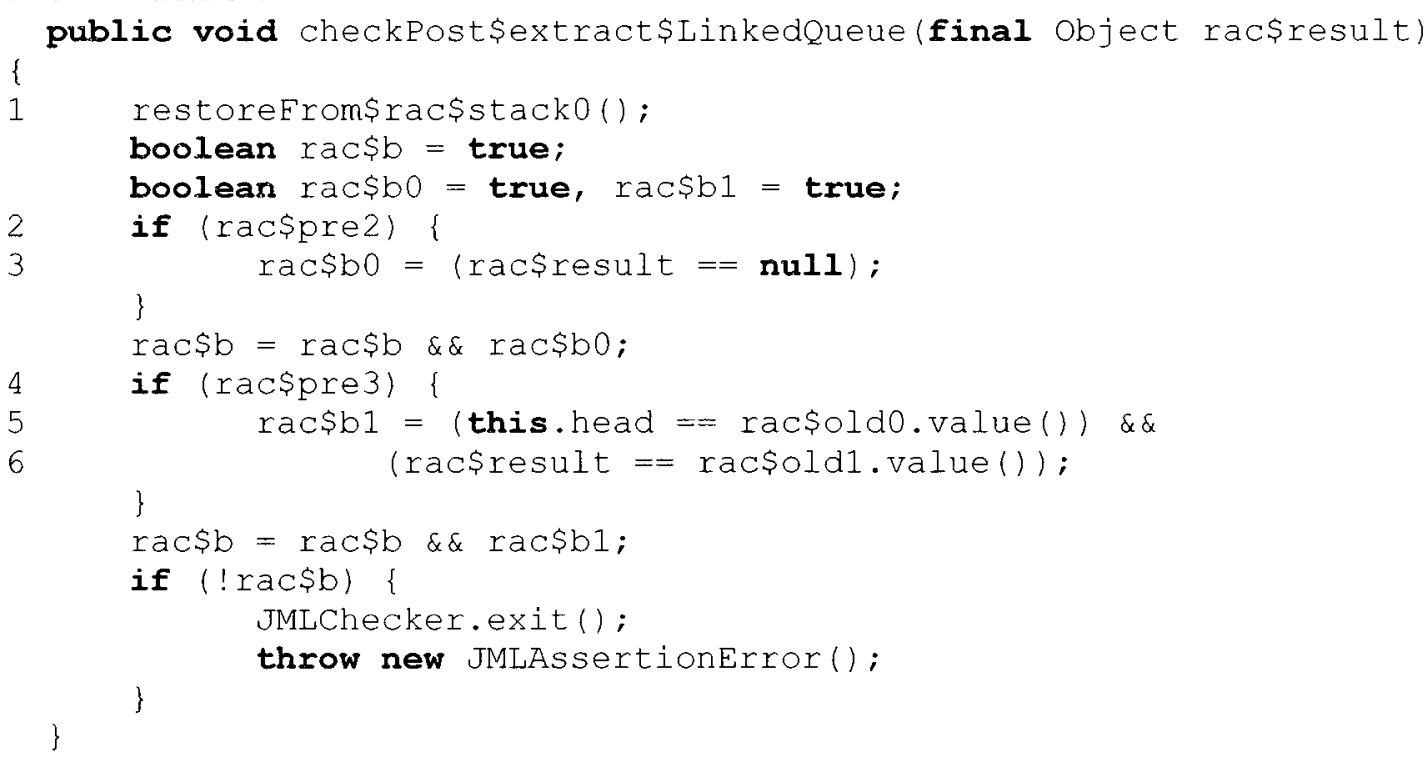

Listing 20: Simplified postcondition assertion method for method extract () of class LinkedQueue. 
Listing 21 shows the specification of interface Channel. It declares two model fields (lines 2-3): A model field (a field with the model modifier) is a field that does not have to be implemented but can be used in a specification as any other field. Model fields allow abstract modeling [21] and play a vital role in information hiding, modular reasoning and behavioural subtyping [12]. Both fields in this example are marked instance: they are fields of the object implementing the interface instead of static fields of the interface (JML allows instance fields on interfaces).

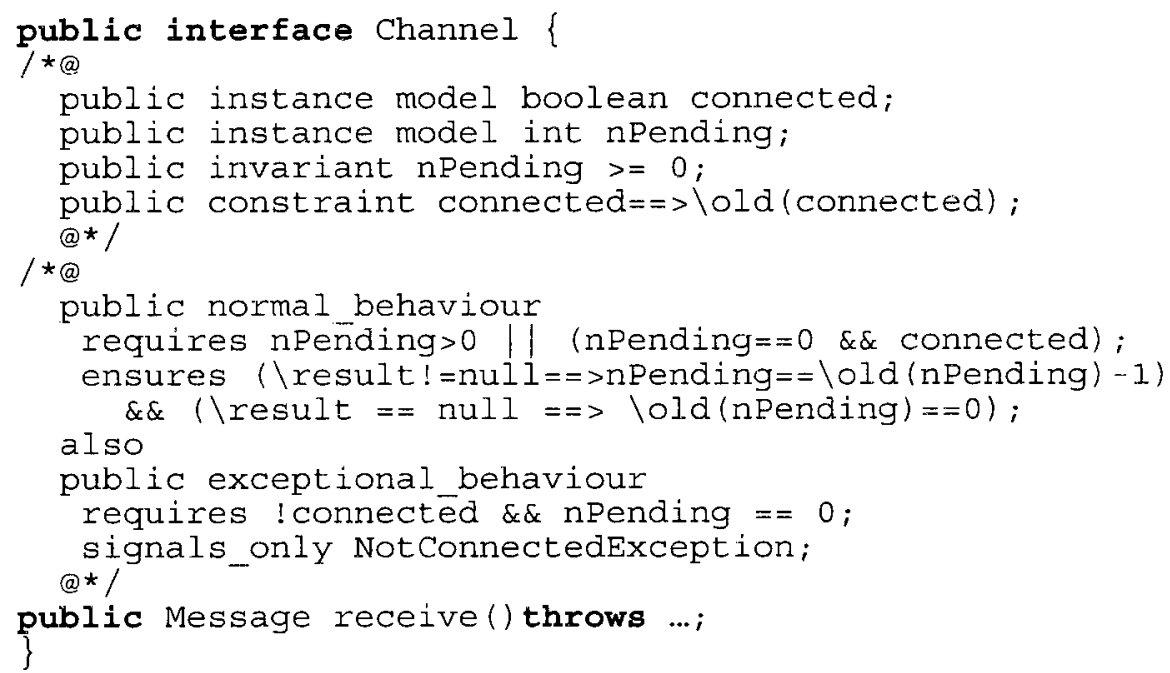

\section{Listing 21: Interface Channel (excerpt).}

Interface Channel can be implemented by class PipedChannel (Listing 22) with the help of a Pipe (not shown). The represents clause maps the value of a model field to an expression based on concrete fields of the class: e.g., the value of model field connected comes from concrete fields closed and remoteclosed according to the Boolean expression in line 3 (Listing 22). 


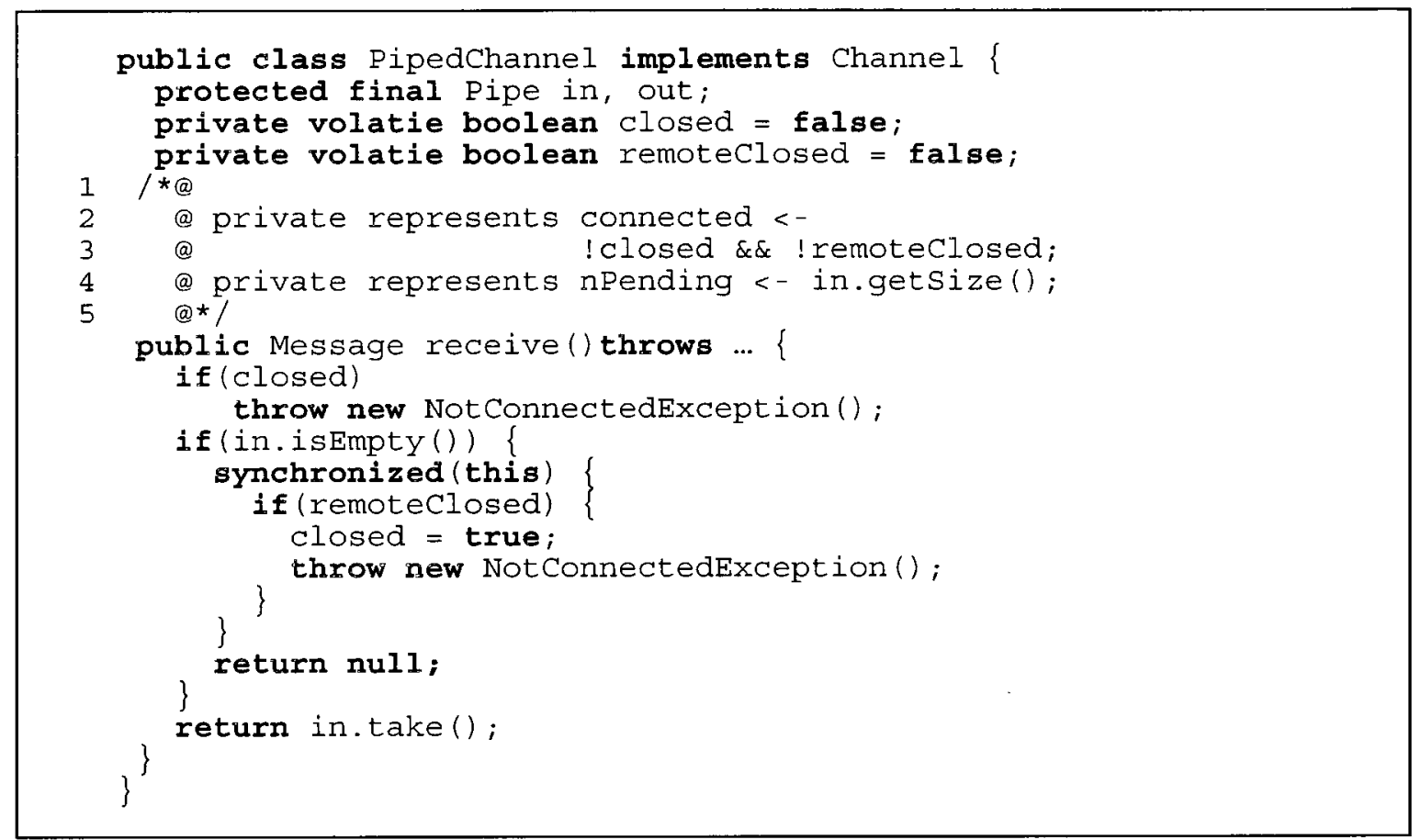

\section{Listing 22: Class PipedChannel (excerpt).}

References to model fields in specifications are translated into calls to access methods declared in the type declaring the model field or, in the case of interfaces, in the corresponding surrogate class. The represents clauses in concrete types are translated into access methods overriding the declarations in abstract supertypes. The body of such methods consists of simply evaluating the expression that determines the value of the model field. Listing 23 shows the access method for model field connected in class PipedChannel, which is the result of translating the represents clause in Listing 22, lines 2 and 3.

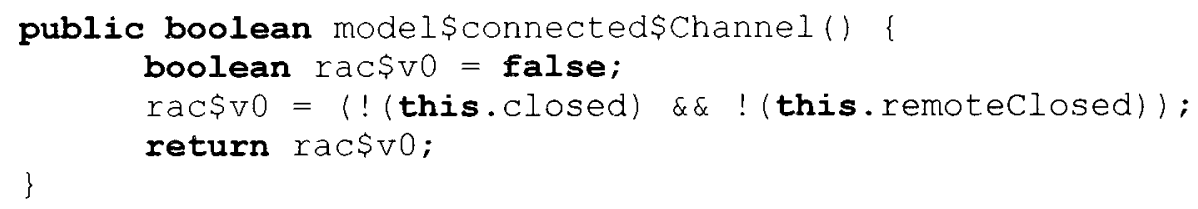

Listing 23: Access method to model field connected in class PipedChannel. 


\subsection{The Runtime Support}

Recall that the state managed by the runtime can be divided in two groups: the local and the global checker state. The former is needed to support recursive calls and to correlate preconditions and postconditions in the same specification case (see Listing 19, Listing 20 and section 2.1). The latter is needed to support calls to Java methods within contracts (line 0 , Listing 18) as well as the checking of locking related predicates. Recursive calls and the correlation of pre- and postconditions are only relevant in the context of the currently executing thread (the current thread for now on) of a method. Similarly, the flag indicating if a Java method is being executed within a contract (i.e. the checker is in checking state) is only meaningful for the current thread. All locking related predicates state properties from the perspective of the current thread. Clauses requires_locked, requires_unlocked, ensures_locked and ensures_unlocked (section 3.2) state if the current thread holds or not a particular lock in the pre- or post-state of a method.

The global checker state was moved from static variables of the JMLChecker class to a thread-local object of the same class thus supporting per thread state keeping. Bookkeeping variables (lines 1,2,3 and 4, Listing 19) as well as the per-method stack (line 5, Listing 19) are transferred to a generated nested class called JMLLocalstate. Instances of this class are kept in thread-local variables as well (one for instance state and another for class (static) state). This has the effect of replacing direct manipulation of state variables with (generated) accessors that internally retrieve the thread-local instance of JMLLocalstate and access the appropriate field from it. The class diagram in Figure 9 exemplifies the case of an instrumented class $x$. Nested class JMLLocalstate contains three fields: rac $\$ 0 l d o$ holds the result of an old expression computed in the pre-state, rac\$pre 1 holds a flag stating if a particular precondition is satisfied, and rac\$stack 0 is the stack variable storing instances of the other two fields to support recursive calls. Instances of the JMLLocalstate class fulfil two roles: the instance and the static (class) local checker state. The association class ThreadLocal represents the fact that the navigation occurs through instances of thread-local variables. Class $x$ declares the accessors to the fields above. They simply manipulate such fields through the static or instance thread-local variables. 


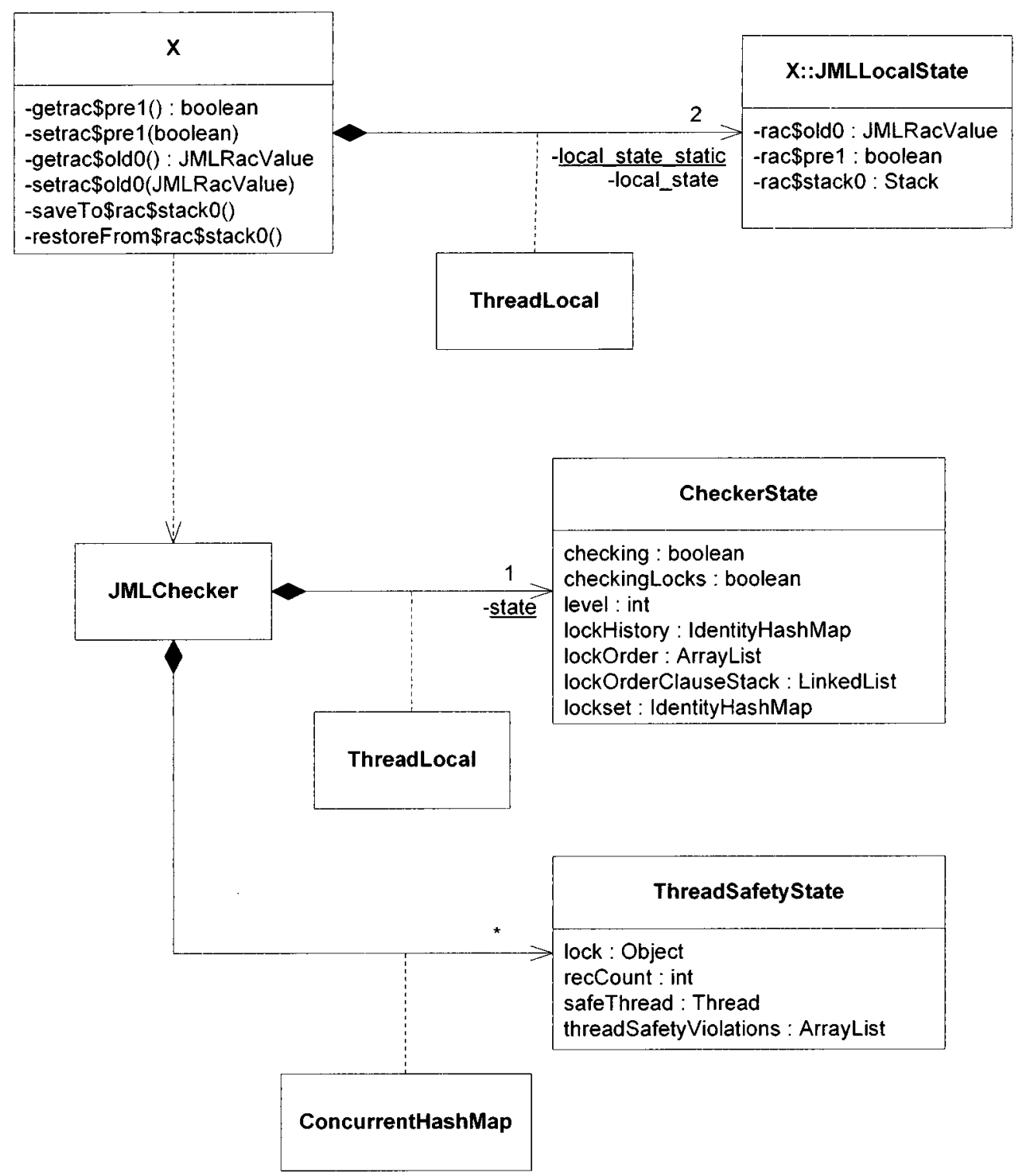

Figure 9: UML class diagram depicting the runtime support for concurrent checking and its relationship to an instrumented class $x$. The members of $x$ in this diagram as well as class $\mathrm{x}:$ : JMLLocals tate are generated during compilation. Class JMLChecker and its components are part of the JML runtime library. ThreadLocal and ConcurrenthashMap are part of the Java runtime library.

The thread-local object that keeps the global checker state was modified to maintain locking related properties for the current thread. It maintains a set of all locks the current thread holds, the order in which these locks have been acquired, and a history of all locks released by the current thread. The first collection supports the evaluation of the 
requires/ensures_locked/unlocked clauses, as well as the lockset expression, which returns the set of locks held by the current thread. The second collection supports the evaluation of the lock_order clause (see section 3.4), which specifies predicates regulating the lock acquisition order of the current thread, as well as the \max expression, which returns the most recently acquired lock by the current thread of the given list of objects. The third collection supports the evaluation of the locks_releases clause, a renamed version of the locks clause introduced in [16] but never implemented in jmlc. This clause specifies the locks a method must acquire and release (see section 3.2). This is shown in Figure 9 by the Checkerstate class. Class Threadsafetystate is discussed in detail in section 4.4. It stores the necessary information to enable the evaluation of thread-safe clauses. The JMLChecker class was augmented with several utility methods to facilitate the manipulation of these collections from generated assertion methods.

\subsection{Safepoints and Wait Conditions}

Two threads interfere when one changes data the other observes. This becomes a problem if, due to an arbitrary interleaving, one thread's perception of the shared data is not true due to a modification made by another thread and it relies on such perception for future computations. A safepoint is any point inside the method body where it is safe to evaluate precondition, postcondition and invariant predicates. A precondition safepoint is a point where it is safe to evaluate preconditions and invariants, and the pre-state predicates of postconditions (i.e. old expressions (see section 2.1)). A postcondition safepoint is a point where it is safe to evaluate postconditions and the invariants. Any method execution path (from the pre-state to the post-state) can have only one precondition safepoint and only one postcondition safepoint. If no precondition (resp. postcondition) safepoint is explicitly specified for an execution path, it defaults to the method pre-state (resp. poststate). In a precondition safepoint, all preconditions, invariants and pre-state predicates are required to be safely evaluated, i.e. they need to be free of interference. In a postcondition safepoint, the postconditions and all invariants are required to be safely evaluated. The requires_safepoint and ensures_safepoint labels demarcate the precondition and postcondition safepoints, respectively. See section 3.1 for details on safepoints. 
Listing 24 shows an example of the use of safepoints (access to last is also protected by a lock on head). At the precondition safepoint (line 15), all the objects referenced by both requires clauses (lines 2 and 6 ) and the contents of the $\backslash$ old statements in the ensures clauses (lines 8-9) are properly protected by locks. At the postcondition safepoint (line 23 ), the field head, present in the ensures clause at lines $8-9$, is properly protected by a lock. Since $\backslash$ result refers to local variable $\mathrm{x}$, which in turn points to an object no longer referenced by the list, it is also thread-safe at the postcondition safepoint. Finally, the object invariant can be safely evaluated both in the pre- and postcondition safepoints since it refers to head, which is properly locked in both places.

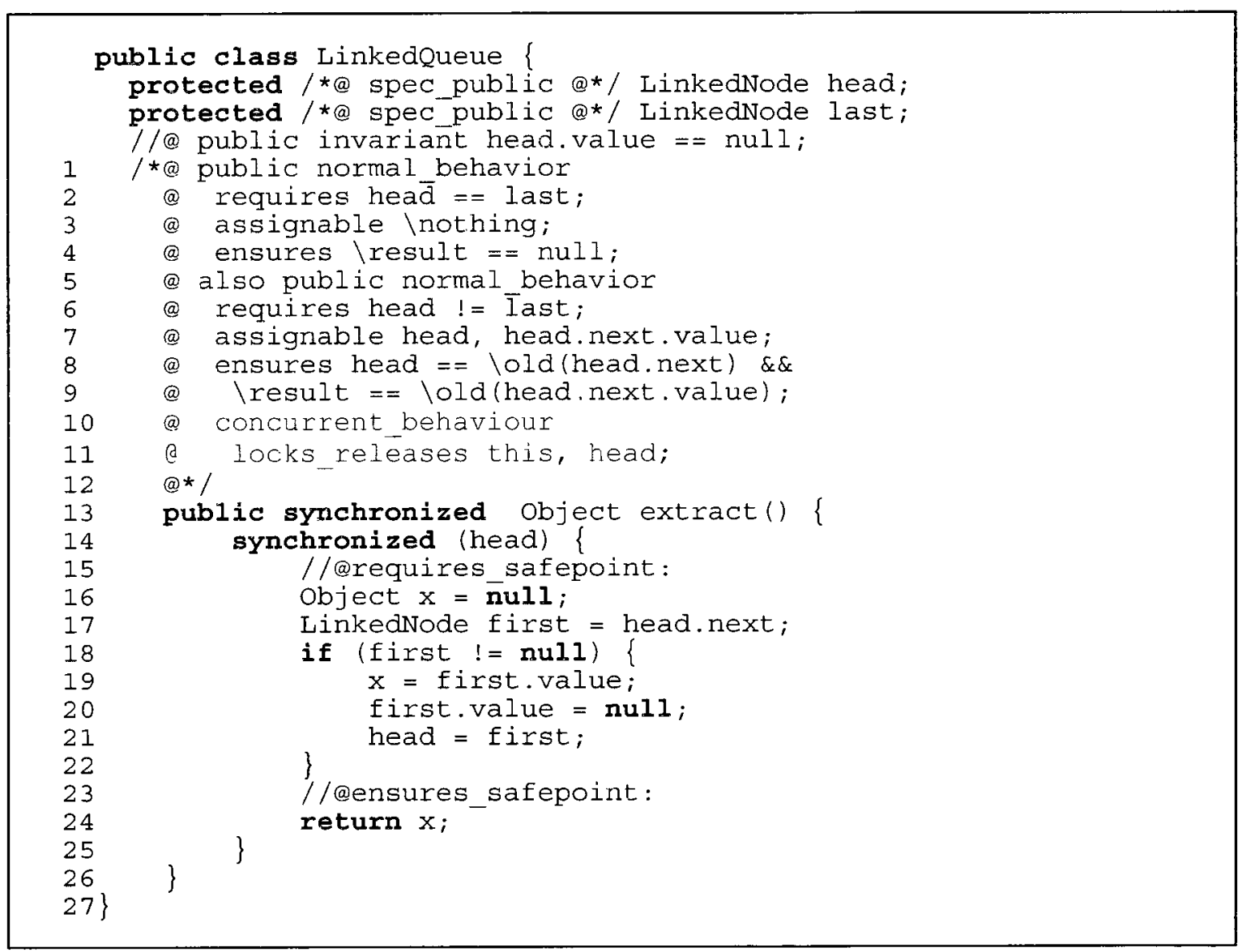

Listing 24: Method extract () of class LinkedQueue using safepoints to avoid internal interference.

There is no significant difference in the assertion methods regarding the support for safepoints. Each safepoint label is translated to a call to a (generated) safepoint evaluation 
method (lines 8 and 9, Listing 29). The content of the safepoint evaluation methods is shown in Listing 25. Comparing them with the original wrapper method (Listing 18), one can see that lines 1 to 3 of the precondition safepoint correspond to lines 0,2 and 5 of the wrapper method, and lines 7 and 8 correspond to lines 15 and 18 of the wrapper method.

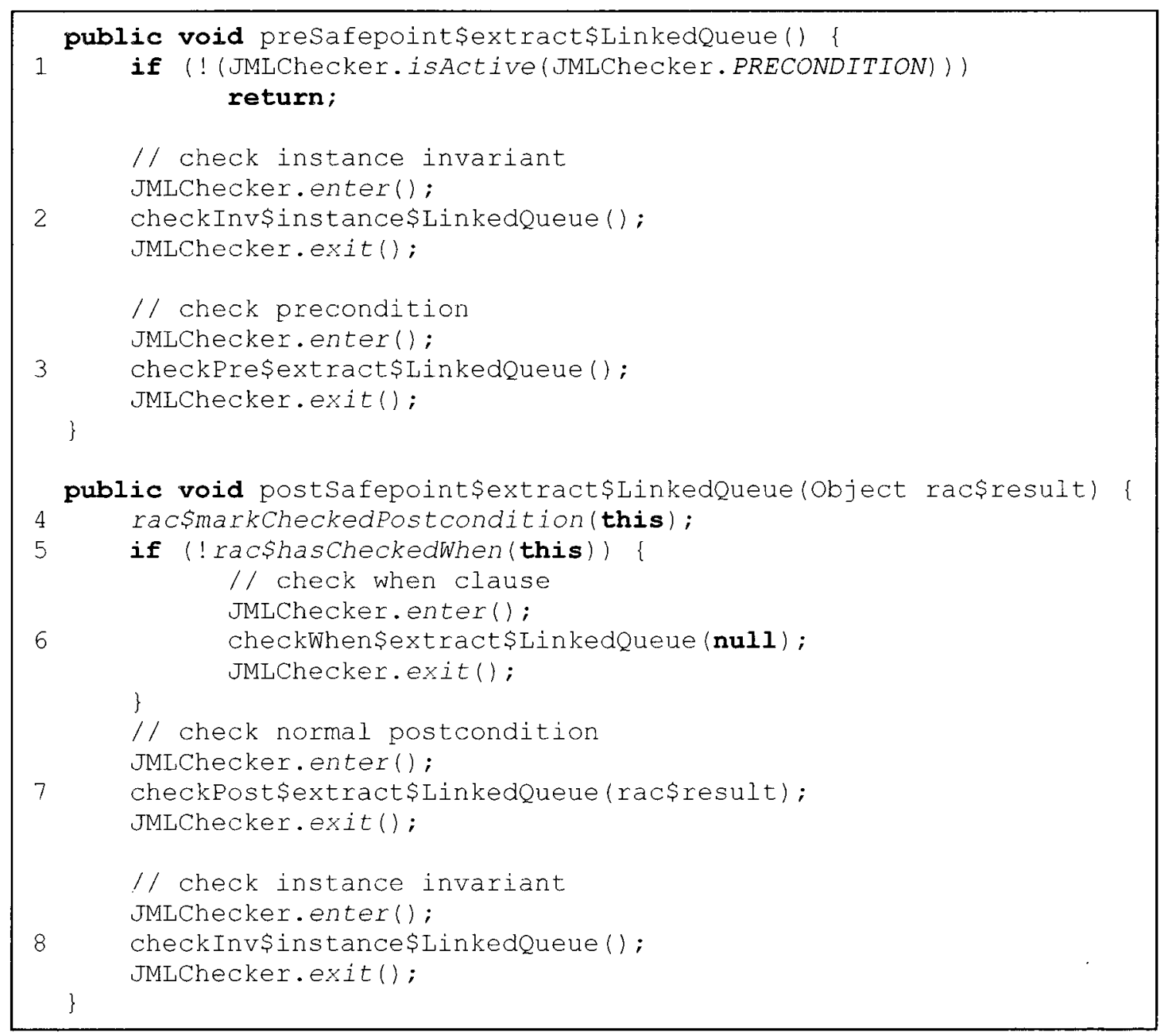

\section{Listing 25: Simplified versions of precondition and postcondition safepoints} methods.

The new (concurrent) version of the wrapper method is shown in Listing 26 . Notice that the precondition and invariant assertion methods are not called anymore (lines 1-6, Listing 18). They are invoked from the precondition safepoint evaluation method, instead. The behaviour is analogous for the postcondition and invariant assertion methods. There is a difference, though. Since the post-state is the default postcondition 
safepoint, the postcondition safepoint evaluation method is also called from the wrapper method (lines 12,13,17 and 18, Listing 26) if it is not invoked within the original method body. Once the postcondition safepoint evaluation method is executed, it sets (line 4, Listing 25) the flag checked in lines 12 and 17, thus guaranteeing that the postcondition assertion method is evaluated only once per method execution. This is not necessary for precondition safepoints since the requirements of definite execution can be checked at compilation time (see section 3.3.1, Definition 2). It is important to note that a call to the precondition safepoint evaluation method is added to the wrapper method (before line 11, Listing 26) only if a precondition safepoint is not explicitly defined to enforce the semantics that a precondition safepoint defaults to the pre-state if no explicit precondition safepoint is declared in the method body. 


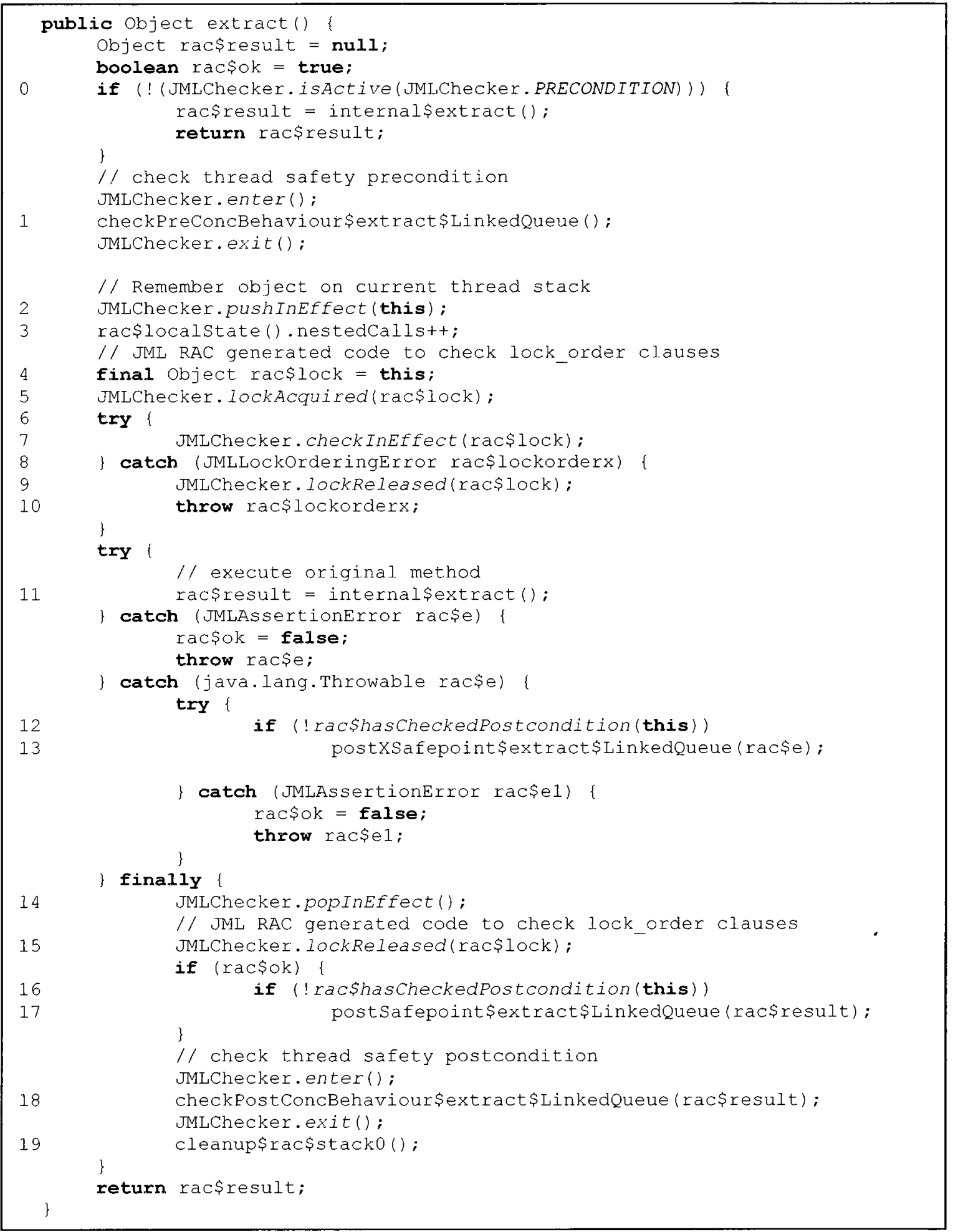

\section{Listing 26: Simplified concurrent wrapper method for method extract () of class}

LinkedQueue. 
JML makes use of the when clause [16] to specify wait conditions. A method blocks until such condition is satisfied. The when clause specifies a predicate that must be satisfied at a particular point of a method execution after which the executing thread is not allowed to block. Such a point is called a commit point and is marked with the JML label commit inside the method. The commit point for a method defaults to the post-state if not explicitly specified. The specified predicate is only required to hold at the commit point; nothing is said regarding its validity afterwards.

The when clause is translated to a wait condition assertion method, which is called from within the method body at the commit point, or from the postcondition safepoint if a commit point is not explicitly specified (lines 5 and 6, Listing 25). The structure of the assertion method is the same as the postcondition assertion method. One difference is that it does not take the method's return value as an argument since one is not necessarily available at the commit point. The commit point implements the same mechanisms as the postcondition safepoint evaluation method to guarantee it is executed only once per method call: the flag (line 5, Listing 25) is set in the method body immediately after the invocation of the wait condition assertion method.

\subsection{Thread-Safety Properties}

Thread-safety properties are specified on a separate facet of a contract called the concurrency control facet as opposed to functional properties like preconditions and postconditions, which belong to the functional facet (see section 3.2 for details). The concurrency control facet is specified via the concurrent_behaviour specification, which can contain one or more of the following clauses:

- requires_locked, requires_unlocked, ensures_locked, ensures_unlocked: These specify the set of lock objects that are held or not by the current thread in the method pre-state and post-state, respectively.

- requires_thread_safe, ensures_thread_safe: These specify that all the objects provided as argument are thread-safe in the method pre-state and post-state, respectively. 
- locks_releases: It specifies the locks this method acquires and releases at any point during its execution.

Each clause takes a comma-separated list of objects (see clause locks_releases in line 11 of Listing 24). Regular objects are treated as monitor locks. A monitor lock is considered acquired while the current thread is within a synchronized method or a block. For instance, in line 16 of Listing 24, both monitor objects this and head are locked. Instances of java.util. locks. Lock are considered locked if the current thread has executed the lock() method more than the unlock(). In Listing 27, lock is considered to be locked by the current thread only in line 2 , since the method lock () has definitely been executed more than method unlock(). These clauses all default to \not_specified. The requires_... clauses must be satisfied in the method's pre-state. The ensures_... clauses must be satisfied in the method's post-state, otherwise an exception will be thrown similarly to a violation of a functional facet clause.

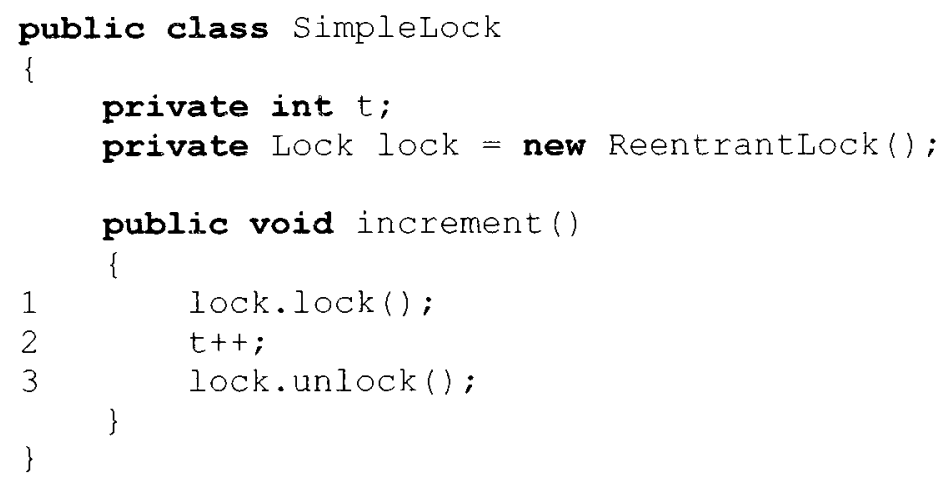

\section{Listing 27: Example of the use of instances of Lock.}

All clauses in the concurrency control facet have an invariant-like specification inheritance semantics: subtypes can only strengthen the concurrency clauses of their supertypes. As with invariants, the effective specification of a type with respect to a particular concurrency control clause is the conjunction of its specification with the effective specification of its supertypes (see section 3.2).

Generating assertion code to evaluate the effective specification of a clause in a type is very similar to the case for invariants. The verification, however, is performed on the 
most specific type of the object receiving the method call instead of using reflection to dynamically invoke the assertion method upwards the hierarchy. This significantly simplifies the process of checking thread-safety properties due to their effect into the state of the runtime (i.e. storing objects being checked in the pre-state and then removing them in the post-state) since one can consolidate the set of objects of interest instead of having to deal with the possibility of processing the same instance multiple times.

For instance, to verify the specification in line 11 of Listing 24 , the evaluation method and the assertion methods shown in Listing 28 are added to the resulting type. Notice that the concurrent precondition assertion method is called by the wrapper method (line 1, Listing 26) as well as its postcondition counterpart (line 19, Listing 26). They are called in the pre- and post-state, respectively. All concurrent evaluation methods follow the same structure, hence the simplicity of the example. Since all concurrent clauses work with sets of objects, conjunction is implemented by the union of their arguments in the evaluation method. It takes a set as an argument, inserts the objects referenced by the clause into it (line 1 of Listing 28) and calls its supertype's version dynamically in case the instrumented method is overriding the parent's method. In this particular example the last step is not applicable since extract () is not overriding a method in its supertypes. This effectively implements the union of the arguments provided to the clause in question with arguments of the same clause in its supertypes. 


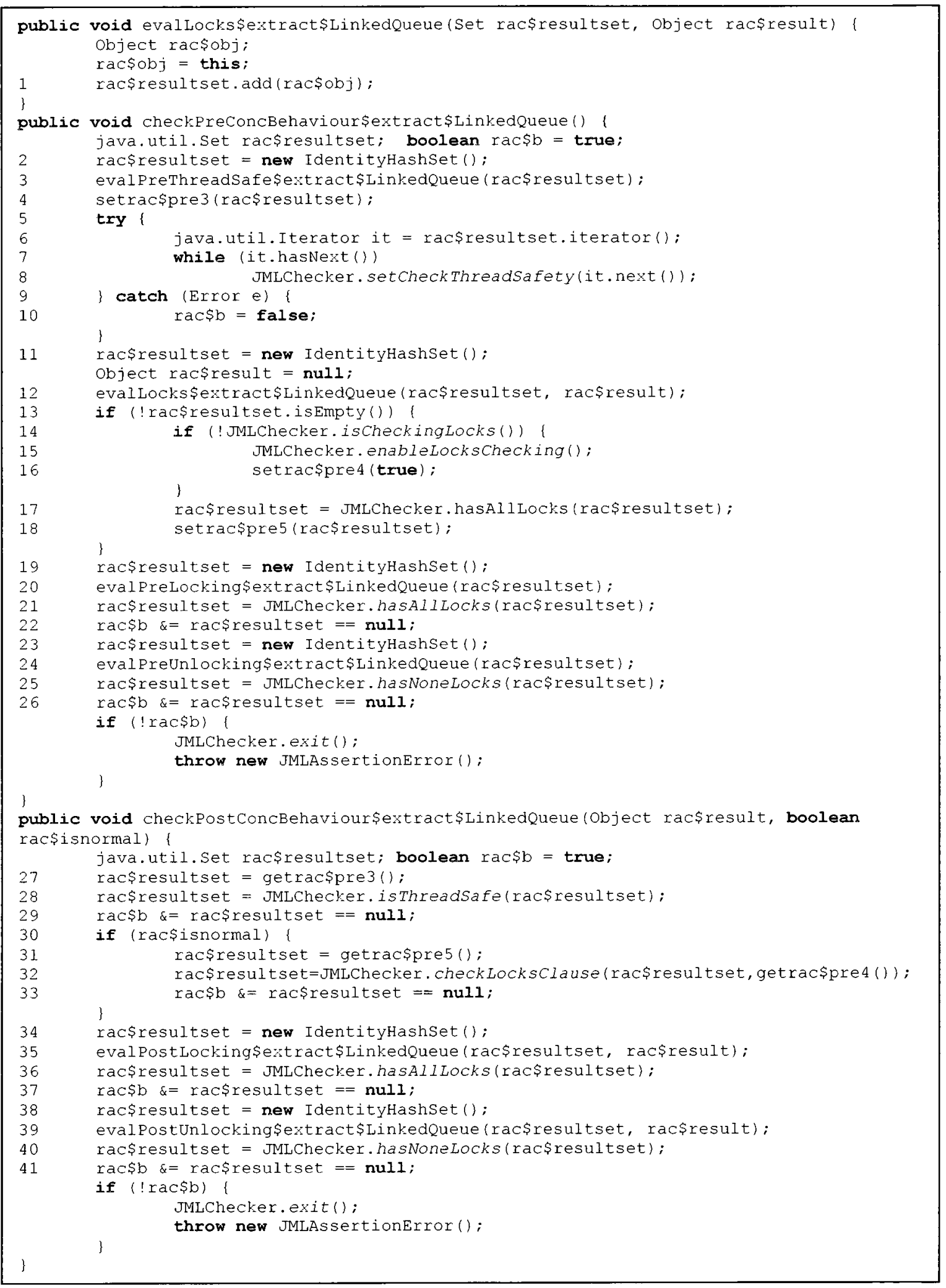

Listing 28: Simplified concurrent assertion and evaluation methods for method extract () of class LinkedQueue. 
The evaluation methods are called from the concurrent assertion methods to determine the objects to be checked. Lines 2 to 10 of Listing 28 mark the objects specified in the requires_thread_safe clause to be checked for thread safety and store them in the method's stack (line 4) to be retrieved in the post-state (line 27) to verify if these objects were indeed only accessed by the current thread (line 28). A similar approach is used for the locks_releases clause (lines 11 to 18 and 30 to 33). The difference is that the first method on the call stack to specify such clause is remembered (line16) so that the lock history can be erased in the post-state (line 32, second argument of the method call), since the locking history needs to be kept between nested method invocations so the outmost locks_releases clause can be properly evaluated. Lines 19 to 26 handle the requires_locked/unlocked clauses and lines 34 to 41 handle the ensures_locked/unlocked clauses.

The locking related clauses rely on the runtime (see section 4.2) to keep track of the locks acquired and released. The runtime, however, just provides thread-safe storage for this information and utility methods to access it. We extended jmlc to instrument method bodies with lock tracking code. Listing 29 shows the instrumented version of the extract () method. Lines 3 to 7 handle the acquisition of monitor lock head (line 14, Listing 24). Line 4 communicates the acquisition to the runtime. This is done prior to the actual acquisition to allow an opportunity to prevent the occurrence of deadlocks by checking lock ordering properties (see section 3.4). Once the monitor lock is released, this is communicated to the runtime as well (line 10). The same mechanism is applied to synchronized methods (equivalent to a synchronized block on this). The instrumentation is performed on the wrapper method instead since it needs to precede lock acquisition (lines 2 to 10 and 15, Listing 26). Lock instances are handled differently. Calls to the several methods to acquire the lock are replaced by a static wrapper method call onto the runtime. For an instance 1 of Lock, a call to method lock () is replaced with the expression JMLChecker.lock(1). The wrapper performs the same tasks as in the case of monitor locks before actually acquiring the lock. Analogously, a call to the unlock () method of 1 is replaced by the expression JMLChecker.unlock(1), which takes care of releasing the lock and communicating this fact to the runtime. 


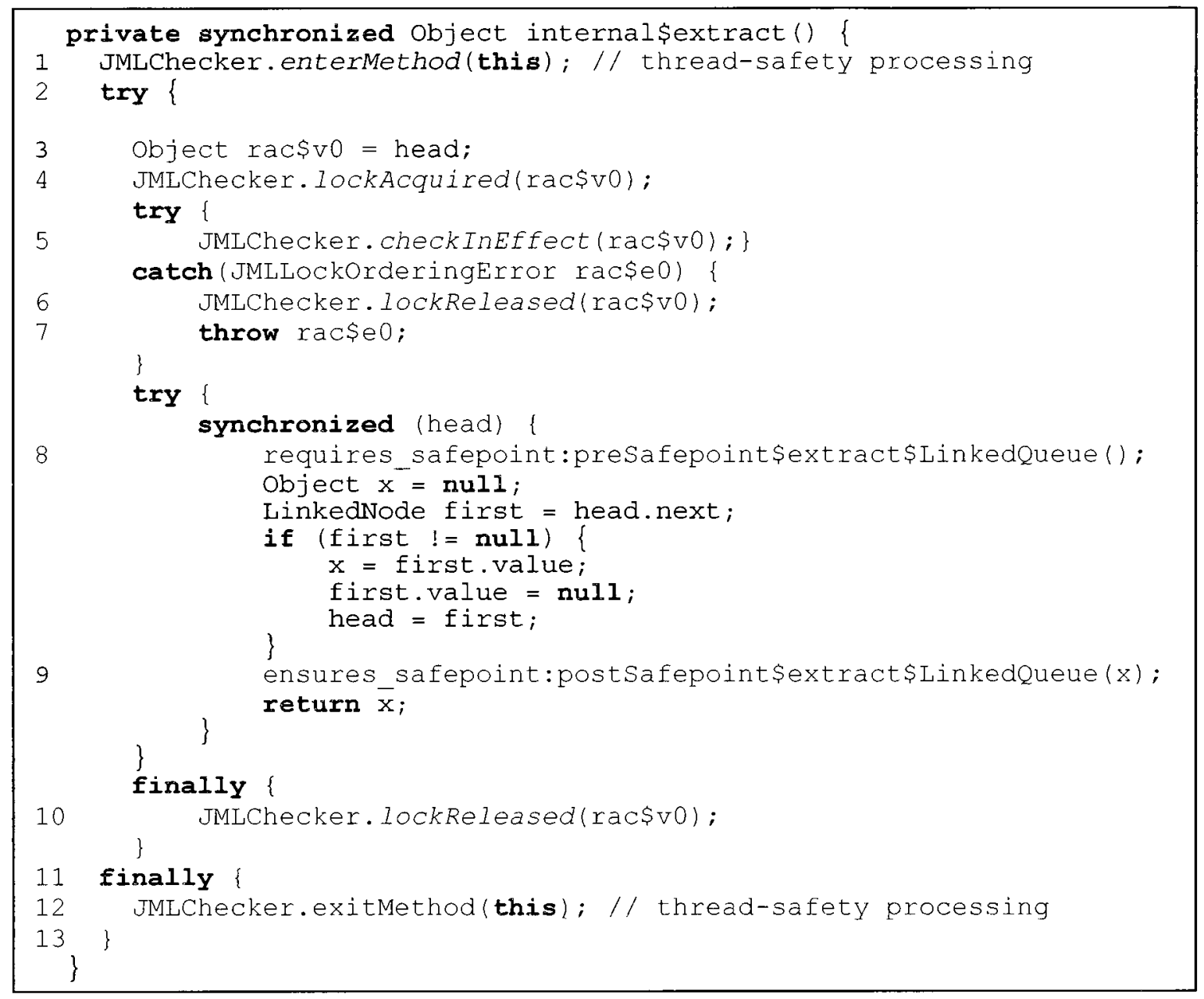

\section{Listing 29: Instrumented version of method extract () of class LinkedQueue.}

Determining the validity of locking predicates is a deterministic process. It is always possible to decide if a predicate is satisfied or not. This is because these predicates reflect the state of the current thread only, i.e. there is no possibility of interference in their evaluation. The dynamic evaluation of thread-safety predicates, however, is fundamentally different. An object is thread-safe if it is local to a thread or access to it is protected by a lock. Current approaches to determining the thread-safety of an object use static techniques for race detection $[19,22]$. These techniques rely on additional information from the developer (e.g. the set of locks protecting an object) to eliminate false positives. Thus, there is always the risk that such information is incomplete. We decided for an approach that eliminates false positives but leaves room for false 
negatives. In a dynamic verification approach (i.e. RAC-based), false positives can prevent the system under test from executing certain parts of the system since violations take the form of exceptions (assertions) being thrown without being caught, which cause a program to terminate execution. We consider to be better to not require any additional (and unreliable) information and allow races to go undetected than the converse, which would cause a premature termination of the system. As discussed below, only races that occur while an object is expected to be thread-safe are detected. This approach transfers the onus of specification to the contract designer, who should clearly state if an object is expected to be thread-safe. Without such specification, the RAC code will not attempt to determine the existence of race conditions involving such object.

The technique we use for determining the thread-safety of an object can be seen in Listing 29. Just after entering the original method (line 1), it calls the runtime to flag that the current thread is about to start executing a method of the given object. It does the same once finished executing the original method (lines 12 to 13). If this object has been marked to be tracked for thread-safety (e.g. line 8, Listing 28) by some other thread executing a method specifying a requires_thread_safe clause referring to this object, then a violation containing the call stack for the current thread is recorded for this object. Upon this other thread reaching the post-state of the method in question it will check if any violations were recorded (line 28 , Listing 28 ) during this method's execution. In such event, an exception will be thrown containing the stack trace of the threads that caused the violation. Such trace serves as a counter-example of the validity of this clause. Otherwise the object being checked is cleared for thread-safety checking causing the runtime to not keep track of method executions for this object.

This technique can also be applied to classes not compiled by jmlc. It is often the case that objects referenced in thread-safety clauses are part of the Java runtime library or other third-party libraries for which source code is not available. We developed a bytecode instrumenter that inserts the calls on lines 1, 2, 11, 12 and 13 of Listing 29 (in bytecode format) into the classes that were not compiled with jmlc. The instrumenter uses the instrumentation interface (part of package java.lang.instrument) to hook into the class loading mechanism and instrument classes as they are loaded by the JVM. 
The runtime supports this technique by keeping a record for all objects being checked for thread-safety (i.e. the thread-safety record) in a concurrent map keyed by object identity. This can be seen in Figure 9, page 80. The thread-safety record is implemented by class Threadsafetystate. Each record keeps a reference to the Thread that is considered safe to manipulate the object, i.e. the thread executing a method specifying a requires_thread_safe clause referring to this object, a list of violations in the form of java. lang.Error instances ${ }^{16}$, and a lock to control concurrent access to the record. The use of a concurrent map avoids introducing an artificial lock contention point for the evaluation of the thread-safety of objects. A concurrent map is designed to be updated concurrently by a predefined number of threads (specified at construction time) and read by any number of threads without contention. A properly sized concurrent map will prevent contention for updates since only the safe thread adds or removes records for an object being checked for thread-safety. A lock is necessary on each thread-safety record to guarantee consistency in case the object is not thread-safe and a violation occurs. This minimizes the contention to cases in which violations indeed occur, which is not an issue since an assertion will be raised and the program stopped.

\subsection{Verifying Lock Ordering Clauses}

Multiple lock_order clauses are compiled into a single assertion method. Individual lock-order-expressions are conjoined in a single predicate. The lock_order clause this $<$ head if added to class LinkedQueue (Listing 24) would produce the assertion method in

Listing 30. Line 1 checks the lock-order-expression against the lock about to be acquired. If acquiring the new lock violates the lock-order-expression, the runtime checking method returns false. Although not shown here, the assertion method handles inheritance like the other assertions methods: via dynamic delegation. The outcome of the supertype's assertion method is conjoined with the local evaluation to produce the final result of assertion method, which simply returns in case of success or throws an exception otherwise.

\footnotetext{
${ }^{16}$ An Error is a special type of exception that indicates an unrecoverable error in the program execution (e.g. out of memory, assertion violation). Exceptions of this type do not need to be explicitly declared in the method signature.
} 


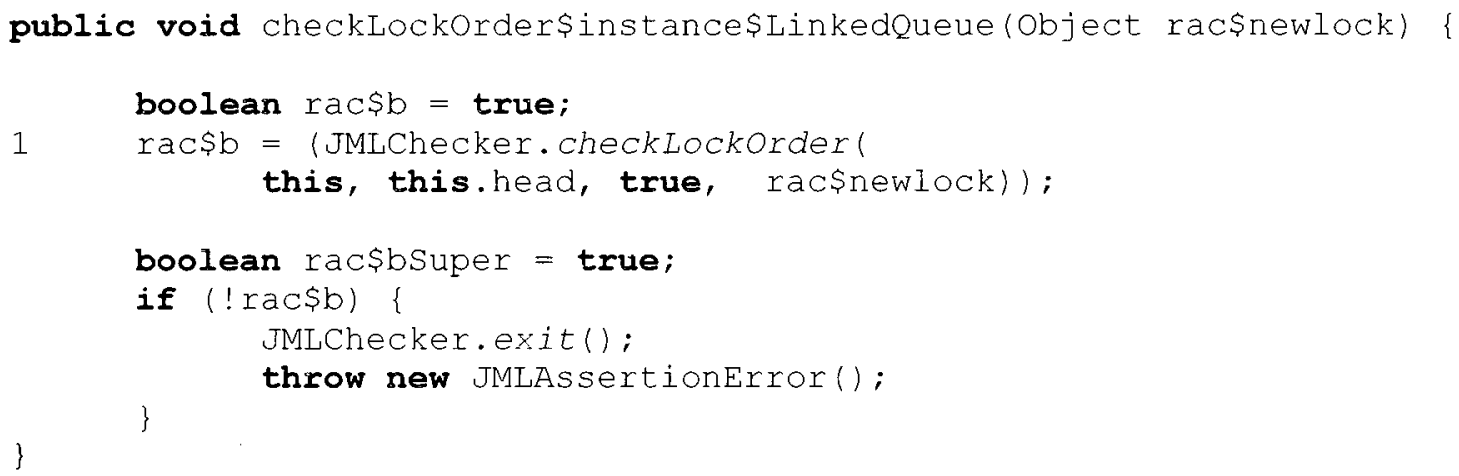

Listing 30: Simplified locking order assertion method for class LinkedQueue.

Assertion methods are called indirectly at every attempt of acquiring a lock. Line 5 in Listing 29 instructs the runtime to check all lock_order clauses in effect that reference lock head. Notice that it first notifies the runtime that the prospective lock is already acquired (line 4). This action is undone and an exception thrown (lines 6 and 7) in case of an ordering violation. The runtime does the same in case of acquiring Lock instances instead of monitor locks (see section 4.4). The runtime maintains a stack of the objects containing the clauses that are in effect in its global state object (see section 4.2). The wrapper method (Listing 26) pushes the target object (this) into the stack (line 2) prior to calling the original method and pops it out (line 14) once the original method terminates. Every object implements a method the runtime calls when walking the stack to check its in effect clauses. This method simply calls the locking order assertion method.

This technique to check lock_order clauses implements the semantics described in the section 3.4. Pushing and popping objects into the stack of in effect clauses reflects the semantics of keeping track of clauses based on methods' activation records. Checking all clauses in effect at every acquisition attempt is accomplished through the runtime indirection described above. The re-evaluation of clauses at checking time by the object declaring it implements the semantics of binding the clause to locks resolved in the context of the object with their latest values. Having the check performed prior to actually attempting to acquire a lock implements the semantics of preventing deadlocks. 


\subsection{Summary}

The techniques used to implement the instrumentation as well as the runtime support were chosen based on the fundamental requirement of not introducing artificial locks or, if unavoidable, minimizing lock contention. Artificial locks have the potential of affecting the interleaving of threads the system can produce. This can, thus, suppress harmful interleavings during the execution of the instrumented system, thus allowing concurrency control issues to go undetected until the production system is executed.

Thread-local variables, used to support several runtime features, are stored in a map local to each Thread instance. Therefore access to these variables is not protected by any locks. The execution of assertion methods at safepoints instead of at the pre- and poststate does not require any additional locks since they leverage the concurrency control mechanisms of the original program to guarantee safety. The use of a concurrent map for thread-safety checking minimizes lock contention. Since these are all the lock contention possibilities, one must conclude that this instrumentation technique does not prevent the occurrence of the thread interleavings that are present in the program in the absence of instrumentation.

This section described the techniques used to generate RAC code for concurrent related clauses as well as the runtime modifications to support it. This demonstrates that the concurrency related constructs introduced can indeed be implemented in a RAC tool. 


\section{RELATED WORK}

Verification of concurrency properties of programs can be divided into three approaches. Static checking uses the source code only (usually augmented with some annotations) to check the validity of certain properties. Dynamic checking uses only information available during runtime execution of the program under test. There are also approaches that combine both techniques. This work concentrates on dynamic checking.

Lipton's reduction algorithm [26] allows one to reason about concurrent programs without considering all possible interleavings. The Lockset algorithm was introduced in Eraser [29] to detect race conditions dynamically and extended for object-oriented languages in [30]. Flanagan and Freund [18] describe Atomizer, a dynamic checker for Java programs. It is based on Lipton's reduction algorithm to define left and right mover operations. It uses the lockset algorithm to keep track of the sharing state of every field an allocated object. Atomizer checks for atomicity, a more fundamental property of concurrent programs. "A method is atomic if its execution is not affected by and does not interfere with the concurrently executing threads." Atomic methods are annotated with the atomic modifier. Atomizer produces instrumentation based on the annotated source code that calls the Atomizer runtime to issue warnings according to the Lockset and reduction algorithms. They report on the use of atomizer on moderate size (up to 90,000 lines of code) programs. They successfully detect errors although the slowdown in some cases is considerable (on the order of 40 times).

Stoller et al [24] describe a combination of runtime and static analysis to check for atomicity. They first execute a type discovery task to automatically "annotate" the source code for race-free types. Then they run a static checker to infer the atomicity types. This step produces atomicity warnings. These warnings are then fed to a "focused run-time checking" phase, which checks for races and atomicity violations limited to the portions that caused warnings. They report on checking for races on a system with 157,000 lines of code with a speedup of $72 \%$ compared with a full run-time checking. They fail to check for atomicity in this system. 
Both solutions have a common limitation intrinsic to dynamic checking: they require that the code be exercised to be checked. Not all interleavings are required to be executed, though. Both techniques are able to discover situations in which atomicity is violated even though the particular violation did not happen just by entering an atomic region. Atomicity checking relies on annotations provided by the programmer to determine the set of locks protecting access to a variable (which is possibly flawed) or the lock inference algorithms used in their place require multiple executions of a method (or block of code in general) being checked for atomicity to make a determination, which does not fit well with a RAC-based approach to verification, in which a predicate is expected to yield an answer in every execution. This work, therefore, does not make use of atomicity as a pre-requisite for the evaluation of contracts.

The Extended Static Checker for Java (ESC/Java and ESC/Java2) $[19,31]$ is able to produce warnings regarding possible race conditions and deadlocks by translating specifications into verification conditions and feeding them and the Java source code to a theorem prover. ESC/Java uses axioms to specify lock ordering predicates. Such predicates are required to be valid at the beginning of a method but not at the end. These predicates can be full logical expressions, including quantifiers. This approach has two disadvantages: it is not sound (it does not impact common practical cases, though) and it is not, in general, easily translatable to RAC code.

Rodríguez et al [16] describe a variety of constructs in JML for dealing with several aspects of concurrency properties. We reuse the when clause and the \lockset expression without modification, used to specify wait conditions and the set of locks a thread holds, respectively. They present solutions to the problem of specifying lock acquisition and thread-safety properties but fail to consider the issue of inheritance. They treat concurrency related properties like regular functional properties (i.e. as part of a method pre- and post-condition). We identify these issues (see chapter 2) and present solutions (see chapter 3 ). We decouple concurrency related properties from functional ones and introduce the concept of facets of a contract, each with its own semantics for specification inheritance. Specifically on the topic of thread-safety, we present a different approach, which does not rely on separate annotations (the monitors_for clause in [16]) 
to determine the thread-safety of an object. Although they propose several constructs, none of them were implemented in the JML toolset. We implement all the constructs we propose on the JML compiler and generate RAC code for them.

Greenhouse et al [32] describe a series of annotations related to the specification of the concurrent behaviour of a Java program. Their annotations are similar to those present in [16] with respect to locking properties and member ownership, and thus suffer from the same limitations. They do not present a specialized construct to state the thread-safety of an object. Their work focuses on the static checking of such properties with an emphasis on the evolution and refactoring of concurrent programs. They do not present a solution to the verification of functional properties in combination with concurrency related properties.

Nienaltowsky and Meyer [33] present an interesting proposition regarding the use of contracts in a concurrent environment. They target SCOOP [34], an extension of the Eiffel language to provide support for concurrency. They transform preconditions referring to separate objects (objects not owned by the current thread) into wait conditions that must be eventually satisfied once the current thread acquires a lock on such an object. Postconditions are treated similarly: locks on separate objects are not released until postconditions are satisfied. The SCOOP model does not allow invariants to refer to separate objects, so their evaluation does not cause waiting. Our proposal reduces to theirs if restricted to the SCOOP model, which specifies that operations on separate objects can only be executed if such objects are arguments of the enclosing routine (the SCOOP runtime is responsible for acquiring the necessary locks prior to executing the routine). This is equivalent as specifying all such objects as $\backslash$ thread_safe and requiring that all locks be acquired prior to calling a method. Their proposal, however, does not contemplate the full intricacies of the Java concurrency model with its synchronized blocks, multiple lock acquisitions and releases inside a method, and no restrictions on method calls on objects accessible by multiple threads. They make a good point in stating that deadlocks can be detected as assertion violations. Our approach treats the problem in a similar way but prevents an actual deadlock from happening by issuing 
an assertion error immediately upon the violation of a locking policy, i.e. prior to blocking.

Jacobs et al $[22,25]$ present a very interesting approach to the problem of concurrency control of aggregate objects. They present a methodology based on object and thread ownership in which a thread must own an object to access any of its fields. This implies that preconditions and postconditions only refer to thread-safe fields. In other words, the internal behaviour of the object cannot be specified in several important cases. Our approach solves this issue with the introduction of safepoints. When thread-safety of objects referenced by contracts is indeed required, our approach can use the concurrent specification constructs to explicitly state such a property so as to allow for its verification.

Without safepoints, all the examples in section 3.1 would have their preconditions reduced to true, i.e. no requirements, since all of them refer to non-thread-safe variables. We see value in being able to specify such properties. The use of safepoints allows the elimination of non-determinism in the specification of a concurrent method. Although a valid specification technique, the use of non-determinism on a RAC scenario defeats the purpose since it would reduce the detail of the specification. The more detail present in a specification, the higher the likelihood of detecting a fault. The techniques for determining atomicity, although very sophisticated, cannot be applied to checking functional properties. Although they can be combined with static techniques that assume a method to be atomic, thus allowing sequential reasoning to be applied to the verification of functional properties, they cannot be easily adapted to work with RAC. This is due to the fact that interference will always be an issue as long as assertion code is executed without proper protection.

This work is the first to allow for the specification and dynamic verification (i.e. runtime assertion checking) of thread-safety properties as well as functional properties in a concurrent environment without requiring atomicity to be established a priori. It is also the first to propose a complete solution to the problem of interference without limiting the 
use of concurrency constructs, thus allowing for concurrent programs in Java-like languages to be completely specified.

The motivation for this work is twofold: to determine if contracts can be used as test oracles in concurrent software during system testing and to determine the effect (and thus produce a recommendation) the contents of a contract have in their ability to detect and diagnose faults.

In [35] Baudry et al describe how to use contracts to generate assertion code. The authors propose metrics to evaluate the benefits of instrumenting contracts. They define robustness (which they later rename vigilance [36]) and diagnosability and apply them to several case studies. The experiments are, however, limited to small programs in which faults are introduced via program mutation [37]. Although the authors apply their model of vigilance to larger systems [36], several assumptions built into the model are derived from their observations of small programs. In [36], the authors refine their diagnosability model and maintain their conclusion that the quality of a contract (i.e. its probability of detecting a fault) is the main factor in determining the diagnosis effort, with contract density (i.e. the number of contracts per program statement) being a negligible factor. It should be noted that the authors do not perform any experiments to verify their results.

Briand et al $[38,39]$ clarify the concept and metric of observability (as a replacement for robustness) and diagnosability. They present a careful analysis of the effect of the complexity (precision) of contracts in the observability and diagnosability and conclude that improvements in the precision of contracts do not affect significantly the detection and diagnosis effort of failures. They also note that the use of hard-coded test oracles is required to detect the remaining faults and that it is more worthwhile to code them than to improve on the contracts precision. As an alternative, they contemplate the use of more test cases to better exercise the contracts in the hope of improving on the detection of faults. Although carefully designed, the experiment is performed on a small system through mutation analysis. It is unknown if the results extend to larger systems presenting significantly more complex behaviour. Furthermore, they restrict their study to contracts built during the analysis phase, which are based on application domain knowledge, not 
implementation knowledge [40]. It is conceivable that more detailed contracts built at design time (and even refined during implementation) would be able to detect more faults and eliminate (or at least reduce) the need for hard-coded test oracles. Finally, their definition of contract precision level does not necessarily reflect a significant change in the effort to either build or check such contract during runtime. This point is explored in detail in the case studies we present.

Both studies are restricted to sequential software and it is, therefore, unknown if their findings are applicable to concurrent programs even if only considering functional properties. 


\section{CASE STUDIES}

This chapter describes industrial case studies that analyze different aspects of the problem of using design contracts as test oracles in concurrent systems and how successful our approach is in dealing with them. It starts by describing the target system and test bed used in all the case studies. It then moves to the individual cases. Each case study is divided in three subsections describing its objective, the methodology used to conduct it and a report and analysis of the results. It concludes with a summary of the results.

\subsection{Target System and Test Bed Setup}

The target system is the Service Activation Engine (SAE) component of the Session Resource Controller product line of Juniper Networks. It is basically a platform to design and deploy value-added services in an Internet Protocol network. It does so by converting service definitions specified as an abstract set of traffic controlling policies for a particular subscriber into device specific policies in the context of the interface such subscriber uses to connect to the network. The SAE currently supports various devices.

The case studies focus on the subsystem that interfaces with Juniper's E-series routers. This subsystem, called the router driver, is responsible for responding to asynchronous notifications from the router regarding the state of each subscriber interface and managing traffic policies for each such interface. Due to the large number of subscribers a router supports, these requests are processed concurrently to maximize system performance. The router driver is responsible for the translation task above, the low-level communication with the router and to ensure correctness in the presence of concurrent processing. It does so by implementing a transactional infrastructure to guarantee ACID (Atomicity, Consistency, Isolation, and Durability) properties of transactions. The SAE is capable of managing approximately 520,000 active subscribers connected to multiple Eseries routers. This amounts to executing approximately 1,500 transactions per second. The complex functionality of the router driver subsystem allows the use of complex functional specification constructs, and its high degree of concurrency with varied and 
intricate concurrency control patterns allows for all proposed constructs to be explored. With respect to code size, the router driver subsystem is composed of 54 classes and interfaces (33509 LOC), all of which are used in a concurrent environment. Of these, 34 present concurrent behaviour.

The SAE communicates with E-series routers through the COPS-PR protocol [41], which is a specialization of the COPS protocol [42] for policy provisioning. COPS defines messages on top of TCP. The SAE also provides an interface for client processes to control the services associated with subscribers connected to the E-series routers it manages. This is the SAEAccess, which is implemented as a CORBA [43] interface. The test bed is controlled by a series of scripts composing the standard test suite used during system testing of the SAE component. The outcome of a test case in the suite is verified by the output (exception thrown or return values) of a SAEAccess operation and/or the contents of certain log files produced by the SAE. This test bed is depicted in Figure 10.

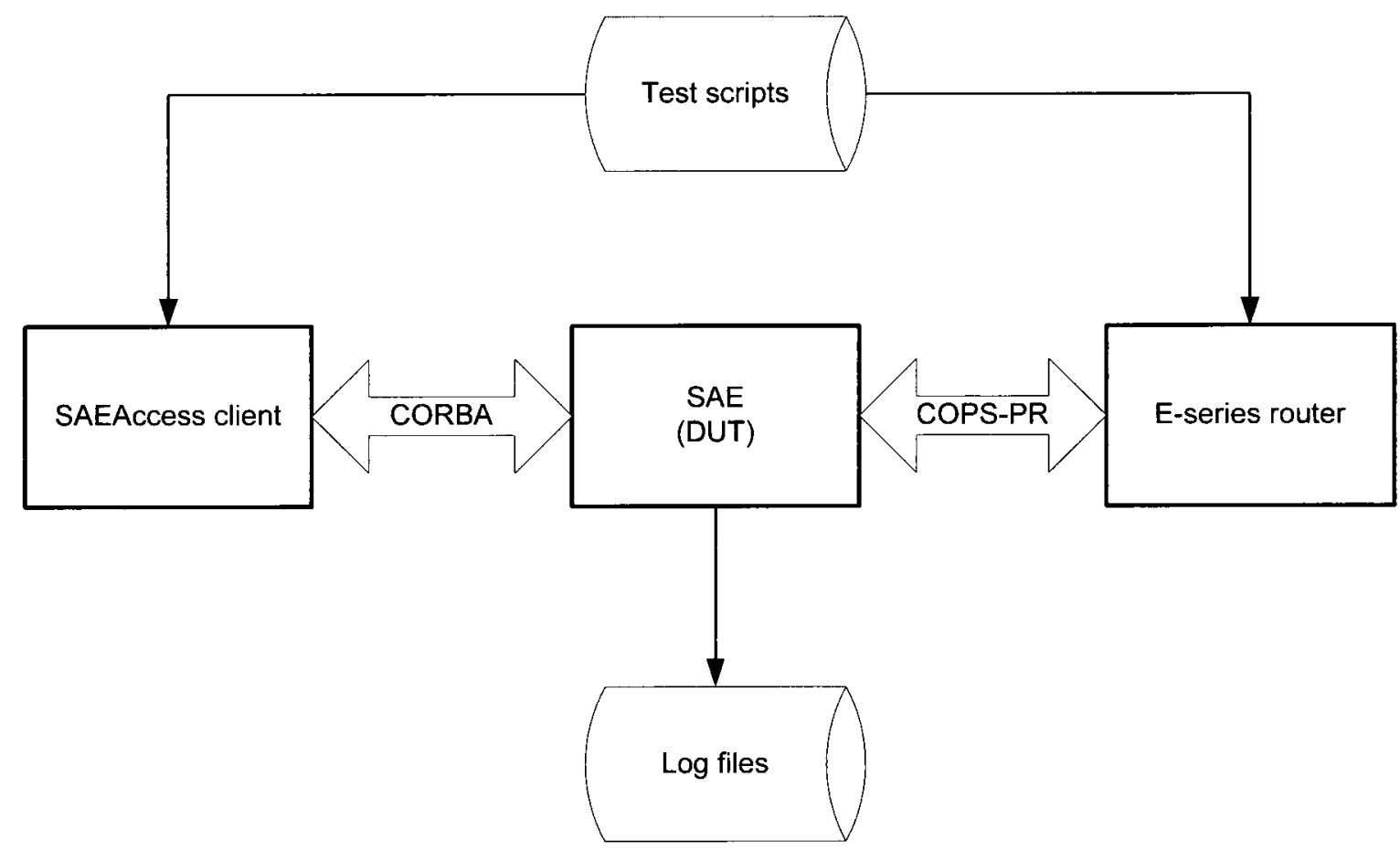

Figure 10: Test bed for case studies. Rectangles represent processes, the wide bidirectional arrows represent inter-process communication protocols, and the curved rectangles represent files locally produced or read by the associated processes. 
The standard test suite is an automated test suite composed of a sufficiently large number of test cases that are required to pass for a version of the SAE component to be released to production. Each test case exercises the SAE through its interfaces and the test case oracle (embedded in the test case) checks return values, parameters and exceptions of operations in the SAEAccess interface against expected values. It also checks the presence or absence of expected contents in the log files produced by the SAE, such as error messages related to the operation performed.

The test suite is built using a black box approach based on test plans derived from functional specification documents of SAE's features. Specific size and coverage parameters of the standard test suite are confidential information of Juniper Networks. However, the key property for the study performed in sections 6.4 and 6.5 can be stated: for every defect present in the defect database there is at least one test case in the standard test suite that exercises the fault caused by such defect thus causing it to manifest as an error in a production system or as an assertion violation in an instrumented system.

The scripts composing the standard test suite take as input several parameters that impact the load imposed on the overall system. Some of these parameters are the rate at which subscribers $\log$ in(out) to(of) the network, the total number of subscribers and types of services such subscribers have. All these parameters are abstracted as a load factor due to their confidential nature. The only property of interest for the experiments described in this chapter concerning the load factor is the ratio between them, i.e. if the load factor in one execution is double the value of another's then the overall load the first execution imposes on the system is double the other's.

\subsection{Specification of an Industrial Concurrent System}

\subsubsection{Objective}

The objective of this case study is to evaluate the capability of the constructs proposed in chapter 3 in combination with the standard JML constructs to specify a concurrent commercial software system. 


\subsubsection{Methodology}

Given the size of its code base and the complexity and diversity of the concurrency control mechanisms necessary to implement a high-performance transaction manager, the target system (section 6.1) can be considered representative of many industrial concurrent systems.

All methods of the classes and interfaces of the target system are specified to the maximum extent possible without modifying them, i.e. the code is considered correct. This restriction is of fundamental importance to eliminate the human factor of tailoring the code to be more amenable to be specified while sacrificing other aspects such as performance or simplicity. For instance, a precondition safepoint requires that only unobservable statements exist between the beginning of a method and itself (section 3.1). A modification of the method body to satisfy such requirement would take the form of removing statements related to logging and tracing or, at least, reordering them. Another example is increasing the scope of a lock by covering more statements in the method body to satisfy thread-safety requirements so that a more precise predicate can be stated. Both situations have the potential of impacting the maintainability and the performance of the system, respectively.

Success is defined by two measures: if a particular clause or construct is essential to specifying the behaviour presented by a particular method and if such clause or construct is able to fully specify it. The former answers the question "is this construct the only option in specifying a particular method's behaviour?" and the latter "can it be used to specify the behaviour in question in the context of such method?" For instance, a method that states a precondition that can only be safely evaluated from within the method body requires that a precondition safepoint be used, since there is no other means of doing so. This answers the first question. If a precondition safepoint can actually be selected inside the method's body (i.e. if it can be placed in a way that there are no observable statements from the beginning of the method to its location) answers the second question. Both parameters need to be present to assess if a particular construct is a useful 
contribution to the ability of JML (augmented with our proposed constructs) in specifying concurrent programs.

\subsubsection{Report}

Table 1 summarizes the usage of the thread-safety constructs we propose. The first column provides a count of the methods considered. The second column lists the number of methods subject to specification inheritance. The third column provides a count of the methods using the thread-safe constructs, i.e. requires_/ensures_thread_safe. The fourth column accounts for the use of locking predicates. All columns show both the total number of methods and the total number of methods that were successfully specified. We consider a specification to be successful if we are able to specify the concurrent behaviour of the method. The first line lists the absolute numbers and the second one the percentage of successful specifications in relation to their respective totals.

Table 1: Statistics on the usage of the proposed thread-safety constructs for the case study.

\begin{tabular}{|c|c|c|c|c|c|c|c|c|}
\hline & \multicolumn{2}{|c|}{$\begin{array}{l}\text { Number of } \\
\text { methods }\end{array}$} & \multicolumn{2}{|c|}{$\begin{array}{c}\text { Thread-safety } \\
\text { spec inheritance }\end{array}$} & \multicolumn{2}{|c|}{$\begin{array}{c}\text { Thread-safe } \\
\text { uses }\end{array}$} & \multicolumn{2}{|c|}{$\begin{array}{c}\text { Lock predicate } \\
\text { uses }\end{array}$} \\
\hline & Total & Succ & Total & Succ & Total & Succ & Total & Succ \\
\hline Total & 347 & 322 & 104 & 102 & 83 & 56 & 42 & 42 \\
\hline Percentage & $100 \%$ & $92.8 \%$ & $30.0 \%$ & $98.1 \%$ & $23.9 \%$ & $67.5 \%$ & $12.1 \%$ & $100 \%$ \\
\hline
\end{tabular}

Let us highlight some of the most important results in Table 1. First, 30\% of all methods make use of specification inheritance on the concurrent facet, demonstrating the significance of representing this situation properly. It also shows that our proposed constructs are able to describe $98.1 \%$ of these cases successfully. Second, $23.9 \%$ of all methods make use of thread-safety clauses independently of specification inheritance cases, of which $67.5 \%$ are successfully specified by the constructs we propose. The cases that could not be specified, including both specification inheritance and thread-safe uses, were due to the fact that objects that perform concurrency control internally (concurrent 
objects) can never be entirely thread-safe but they can be piecewise thread-safe. An object is piecewise thread-safe if it can be partitioned into groups of methods or fields that do not interfere within such a group and for which concurrency control across groups is taken care of internally by the object. A producer-consumer scenario in which up to one producer and one consumer threads are allowed to execute disjoint sets of operations on an object is one example. It is currently impossible to specify such behaviour on the concurrent facet of an object. The code of piecewise thread-safe objects can, however, be reorganized as to split these groups of methods into different objects. Coding guidelines could also be put in place to explicitly forbid or minimize the use of this pattern. This limitation is, therefore, not severe. Third, this table shows that all cases involving the use of lock predicates were correctly specified by our constructs.

\section{Table 2: Statistics regarding the use of concurrency clauses not related to thread-} safety

\begin{tabular}{|r|c|c|c|c|c|}
\cline { 3 - 6 } \multicolumn{1}{c|}{} & \multirow{2}{*}{$\begin{array}{c}\text { Number of } \\
\text { methods }\end{array}$} & $\begin{array}{c}\text { when } \\
\text { clause } \\
\text { uses }\end{array}$ & $\begin{array}{c}\text { locks_releases } \\
\text { clause uses }\end{array}$ & \multicolumn{2}{|c|}{ Safepoints uses } \\
\hline Total & 347 & 10 & 102 & 101 & 91 \\
\hline Percentage & $100 \%$ & $2.9 \%$ & $29.4 \%$ & $29.1 \%$ & $90.1 \%$ \\
\hline
\end{tabular}

Table 2 summarizes the uses of other concurrency clauses. The third column displays the total number of methods using the when clause. The fourth column displays the total use of the locks_releases clause. The fifth column displays the total number of methods that successfully use safepoints. The first line lists the absolute numbers and the second one the percentage relative to the total number of methods. Table 2 shows that the when clause despite being used by a small number of methods is required for a complete description of the system. It also shows that the locks_releases clause is used by a significant number of methods thus emphasizing its importance. With respect to both clauses, $100 \%$ of eligible methods were successfully specified. More importantly, this 
table shows that $29.1 \%$ of all methods required the use of safepoints to be correctly specified and that our proposed construct succeeded in specifying $90.1 \%$ of these cases.

The major limiting factor preventing the use of safepoints in $9.9 \%$ of the cases was the fact that it is not always possible to have them in the method body due to the requirement of not allowing observable statements to happen before (after) a precondition (postcondition) safepoint. In such cases, only limited predicates can be safely evaluated. However, those cases that could not be specified could have been so by reorganizing the method's code to allow for the placement of safepoints. Such reorganization consists of simply moving observable statements (in their vast majority logging and tracing calls) to be located after the precondition safepoint. This reorganization does not change the overall functionality of the method. It reorders statements within the method that present no causal dependency between each other. In case one is implementing a method for the first time or through subsequent evolution, it is trivial to avoid placing the vast majority of observable statements as to enable the correct placement of safepoints given one can reorganize code already written. The only case, thus amounting to less than $1 \%$ of the safepoints use cases, in which the statement reordering was not possible was for the message queue manipulation method that correlates responses from the router to requests originated on the SAE. It could not be specified because the correlation is performed inside a synchronized block and such block is surrounded by observable statements that could not be relocated thus forbidding the use of safepoints inside it to enable the verification of its precondition.

\subsubsection{Summary}

This case study shows that our proposed constructs are not only essential to the proper specification of concurrent programs but that they are also capable of specifying most behaviour. The thread safety constructs are not able to specify what we call piecewise thread-safe behaviour of objects, which amounts to $15.5 \%$ of the eligible cases. The use of safepoints to specify properties of a concurrent system proved to be not only essential but also applicable to the vast majority of cases. Those cases that could not be specified could have been so, with only one exception, by reorganizing the method's code to allow 
for the placement of safepoints, thus confirming that safepoints are capable of specifying concurrent object-oriented programs.

The definition of successful specification used in this study is subjective since it depends on the expertise of the experimenter evaluating its completeness, which is a threat to the validity of the conclusions. There are, however, several factors that mitigate the risk of the experimenter erroneously considering a specification successful (i.e. a false positive). Specifications contradicting the code can be easily identified by executing an instrumented version of the system. Since the system is considered correct, any assertion violations encountered indicate an erroneous specification (this is a step in the experimental procedure described in section 6.3.2). Incomplete specifications, however, cannot be automatically identified. This risk is mitigated by two factors: the specifications are derived directly from the source code, and the experimenter is an expert on the system being specified. False negatives are virtually impossible since considering a specification not successful is simply a matter of finding an example of behaviour in the code that cannot be reflected in the specification.

\subsection{Applicability of Runtime Assertion Checking in the Verification of Concurrent Commercial Software}

\subsubsection{Objective}

The objective of this case study is to evaluate the applicability of the instrumentation techniques described in chapter 4 to produce an instrumented version of the target system that can replace it during the system test phase.

\subsubsection{Methodology}

The target system is a representative concurrent system and specified following the methodology described in section 6.2.2. Therefore, the instrumented system should present the same external behaviour as the production system. We name this factor indistinguishability. The instrumentation techniques introduce extra processing steps and 
require more data to be stored for the purpose of verifying the validity of the contracts. Therefore, the instrumented system is expected to consume more resources (CPU, memory and persistent storage) than the production system. We name this factor runtime overhead. These two factors are considered independently.

The runtime overhead is analyzed by comparing the CPU utilization, the heap utilization, the size of class files and the amount of the permanent generation memory ${ }^{17}$ between the production and the instrumented versions of the target system when submitted to the standard test suite. Load parameters are adjusted so that CPU utilization remains below $80 \%$ to avoid any saturation (i.e. attempting to consume more than $100 \%$ of CPU), which would invalidate the comparisons between the two versions of the system due to loss of linearity. Success is defined by the values of the resource parameters on the instrumented version remaining bounded by a constant factor ${ }^{18}$ of the production version with the standard test suite producing the same results under the same load conditions. This criterion is fundamental to enable one to adjust load parameters applied to the instrumented system and have it behave as a scaled version of the production system.

Indistinguishability is analyzed based on the behaviour of an instrumented version of the target system compared to its production version. Success is defined by the following:

1. The instrumented and the production versions of the target system pass the standard test suite (as for the runtime overhead factor).

2. Faults introduced in both versions cause the same failures when submitted to the standard test suite, i.e., the test cases that fail and succeed are identical for both versions. The faults introduced originate from Juniper's defect database and are related to the router driver subsystem. Moreover, the faults considered are only those found during the system test phase of the product and only through a period

\footnotetext{
17 The permanent generation is the area of memory in a JVM reserved for storing the in-memory representation of the loaded classes.

${ }^{18}$ For a given function $R(t)$, representing the usage of a particular resource over time, there is a constant $c>$ 0 such that $R_{I}(t)^{\prime}=c R_{P}(t)^{\prime}$, where the subscripts $I$ and $P$ indicate the instrumented and the production versions of the system, respectively.
} 
for which the feature set of the subsystem remained the same. This amounts to a total of 129 faults.

3. A qualitative analysis of the instrumentation techniques give a high degree of confidence that thread interleavings present in the production version are also present in the instrumented version. The instrumentation techniques are analyzed as code transformation rules that transform contracts or code into instrumented code (see sections 4.3, 4.4 and 4.5 for the rules and section 4 for a general explanation). Each rule is analyzed with respect to the introduction of locks, which is the only ${ }^{19}$ way to exclude thread interleavings from a program in which synchronization is performed solely through the use of locks (see section 6.3.4). In the only case where a translation rule introduces a lock we show that contention is very unlikely.

\subsubsection{Report 1: Runtime Overhead}

Program size is measured in two ways: class file size and permanent generation size. The class file size is the number of bytes of the bytecode representation of a Java class or interface according to the Java Virtual Machine Specification [44]. The purpose of this metric is to determine the increase in the amount of persistent memory necessary to hold the instrumented program uncompressed. The permanent generation is the area of runtime memory of a JVM dedicated to holding the runtime representation of a class or interface. The purpose of this metric is to determine the increase in the amount of runtime memory necessary to load the class file into memory.

Both metrics are important to understand the system requirements to execute the instrumented program in conditions equivalent to the original system. Although permanent generation size is not a usual issue with current server platforms, it may prove to be a challenge for memory constrained devices such as mobile phones. The size of the permanent generation has implications on the class loading time as well as on the garbage

\footnotetext{
${ }^{19}$ Even though the timing of thread executions may change due to the instrumentation overhead, thus affecting their interleavings, a sufficiently large number of executions will eventually exercise all possible interleavings given the non-deterministic nature of thread scheduling. This is the most popular technique to detect concurrency control issues.
} 
collector (GC) behaviour. For instance, as the used amount of permanent memory approaches the total allocated space, full garbage collections (Full GCs) happen more often. A Full GC is the only type of collection that allows for the collection of unreferenced class objects. A Full GC also includes a full collection in the old generation in an attempt to free as many unreferenced objects to allow for higher chance of collecting more class objects. Full GCs cause all threads of a JVM to pause until the GC finishes its work. This is not normally an issue in JVMs with a small old generation (i.e. a small heap) but it can cause pauses in the order of tens of seconds for systems with more than $8 \mathrm{~GB}$ of configured heap. Such unexpected delays will cause the program to run in an environment very different from the intended one in terms of timing and thus the results obtained during testing would not be representative.

The graph in Figure 11 compares the incremental class file size for the instrumented system using the multi-threaded (MT) modifications with the original JML instrumentation (sequential) as a function of the number of methods in each class, since most of the translation rules translate one method specification into multiple methods implementing such specification (see chapter 4). Interfaces were not included in this comparison because they bias the results since their instrumentation process does not generate several constructs present in classes and there are not enough interfaces (only 8) in the population to perform a separate meaningful analysis. The incremental file size is the size of the instrumented class file minus the size of the non-instrumented class file size. The population size is 59 , which includes inner and nested classes. 


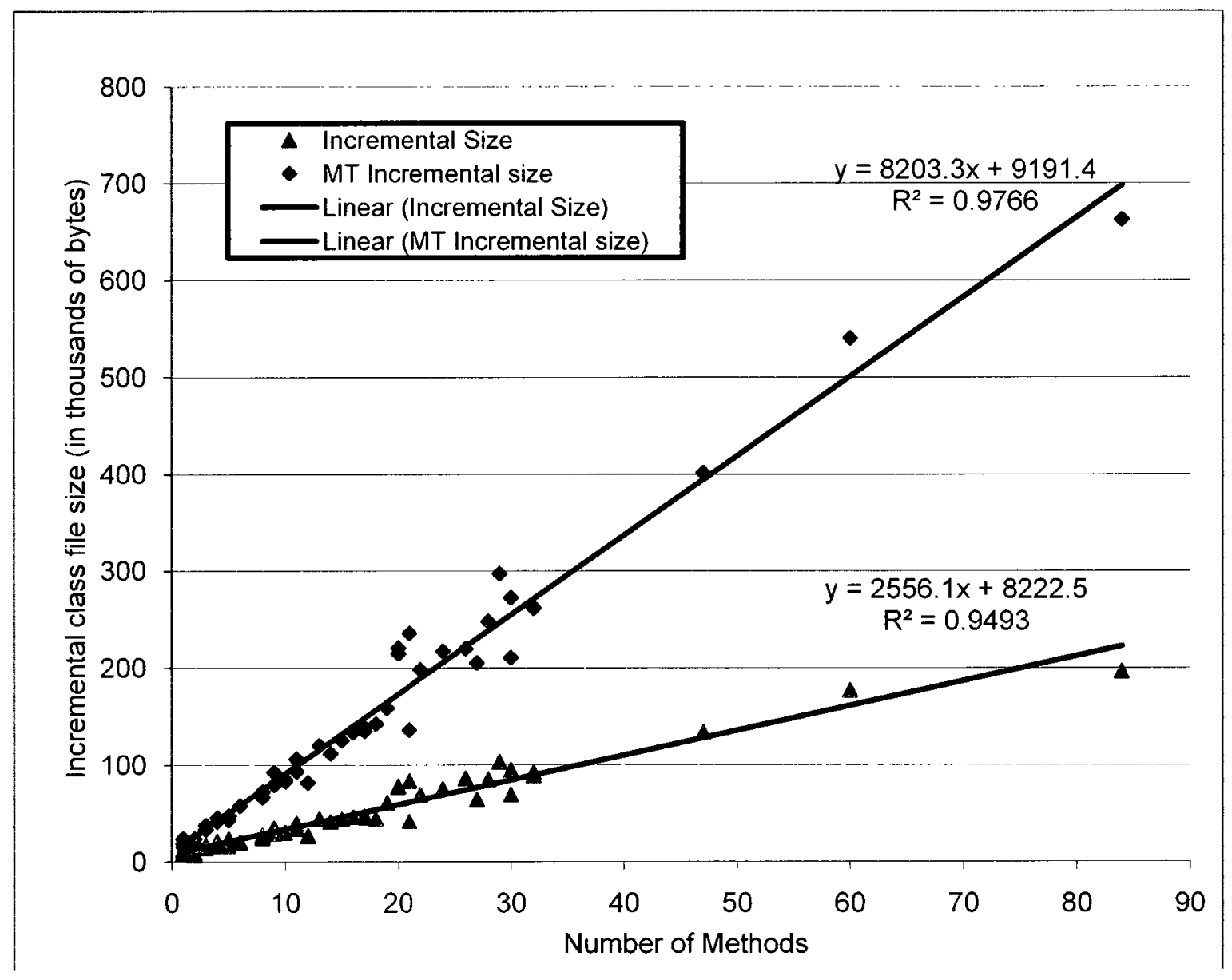

Figure 11: Graph comparing the incremental class file size for classes instrumented using the technique for multi-threaded and sequential programs as a function of the number of methods in the non-instrumented class. The solid lines represent linear regressions for their respective series.

Analysing the linear regressions of the two data sets (MT versus sequential) allows us to draw three conclusions. First, that the instrumentation technique for multi-threaded programs produces considerably more bytecode than the sequential technique, approximately $8 \mathrm{kB}$ per method of the non-instrumented class compared to $2.5 \mathrm{kB}$. This is due mainly to the addition of the concurrent clauses evaluation methods and the introduction of accessors to local state variables (see section 4.2), which are accessed directly in the sequential case. Although these methods are very simple, any method requires a minimum space in the constant pool of a class (see [44]) thus causing this increase in its size. The second conclusion is that the complexity of the checking code 
does not significantly affect the size of a class since the population of classes used in this comparison have methods with complex contracts consisting of multiple specification cases each with predicates with several conjuncts as well as classes with methods with no contracts at all and the spearman correlation coefficient is 0.99 for the multithreaded case and 0.97 for the sequential case, which indicates a strong dependency of the incremental class size on the number of methods and, consequently, a small dependency (i.e. less than $1 \%$ and $3 \%$, respectively) on the contents of the contracts and other unaccounted factors. The third conclusion is that the multi-threaded instrumentation process introduces a minimum of $9 \mathrm{kB}$ of infrastructure that is not dependent on the number of methods whilst the sequential technique introduces about $8 \mathrm{kB}$. Such small increase is due to the creation of an inner class to hold the state of the checker in a thread-safe manner (see section 4.2). This allows us to derive the following formula to determine the total class size (in kilobytes) of the instrumented version of the system based on the number of methods of the classes in the production version of the target system:

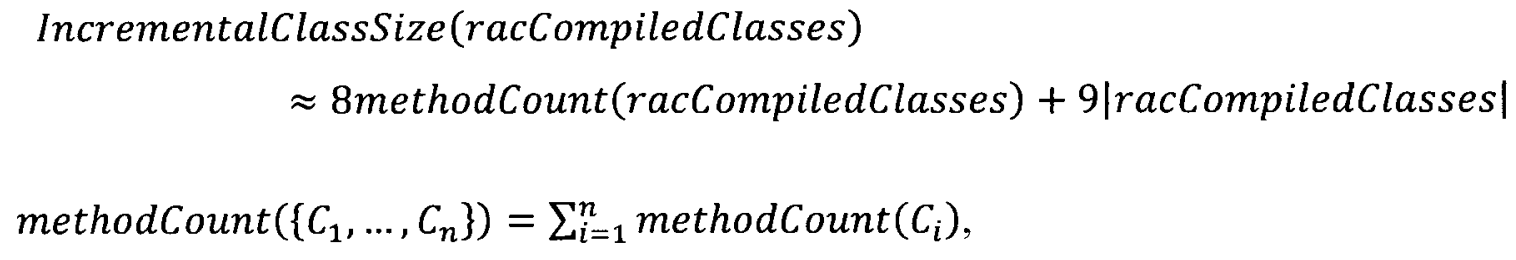

where racCompiledClasses is the set of classes compiled with the JML compiler, and methodCount(.) returns the number of methods in a given class. IncrementalClassSize returns values in $\mathrm{kB}$. The average magnitude of relative error observed between the calculated and the observed incremental class file size was $10 \%$.

The total number of loaded classes in the instrumented version increased by 855 . Of these, only 254 are a direct result of the instrumentation process. There are 63 classes in the RAC runtime. This is a constant. 159 classes are a result of the instrumentation of top level and nested classes but not inner classes. For each such class (53 in total), three classes are generated: one to hold the state of the checker (see section 4.2) and two thread-local variables pointing to different instances of the checker state (one for instance related state and one for class related state, i.e. static members). Nested classes share the infrastructure of the top level class and thus do not cause any classes to be created as a 
result of its instrumentation. 32 classes are a result of the instrumentation of interfaces ( 8 in total), for which one surrogate class is created (see section 4.1). Each surrogate class declares three classes to hold the checker state analogously to top level classes. The remaining 601 classes are synthetic classes (i.e. generated by the JVM) for a variety of purposes like type and method accessors. These are, however, very small compared to the instrumented types.

In terms of runtime memory consumption, the increase in the permanent generation size was about 29.3MB. The total incremental class size, including all interfaces, nested classes and inner classes was about 7.542MB, which corresponds to an average ratio of 3.9 between the increase in the permanent generation and the class file size. This allows one to determine the amount of memory to reserve to the permanent generation based on the number of methods of the classes in the production version of the target system:

IncrementalPermGenSize(racCompiledClasses) $\approx 3.9$ IncrementalClassSize(racCompiledClasses)

where racCompildClasses is as above and IncrementalPermGenSize is the incremental size of the permanent generation memory area used by the instrumented version of the system in $\mathrm{kB}$. Given that the permanent generation size depends only on the class file size and that the latter is a generic conclusion the formula above is also generic.

We also measure the increase in memory footprint (heap) and CPU utilization. The heap is the memory area in the JVM where all dynamically allocated objects reside. The heap needs to be carefully dimensioned to avoid not only failures in allocating objects but also to avoid excessive load on the JVM garbage collector (GC), which would cause a significant increase in CPU utilization, possibly reducing the amount of cycles available to execute the application itself.

Figure 12 and Figure 13 compare the heap and CPU utilization, respectively, between the production and the instrumented versions of the target system subject to the same test 
suite. The test suite consists of a ramp-up phase, in which subscribers are logged into the system (approximately the first 20 minutes), and a steady-state phase in which several operations are performed (the remaining time). The duration of the test is about two hours. The overall load factor (a number controlling several parameters of the test suite including the total number of subscribers and the rate of subscriber login and logout) was chosen as to cause $30 \%$ CPU utilization during the steady-state phase of the suite for the instrumented version of the system. This was necessary to guarantee that the CPU utilization would almost never go beyond $80 \%$ to ensure that CPU was never a contention point. CPU utilization above $80 \%$ increases the chance of having significant periods during which the CPU utilization is $100 \%$. During such periods, the CPU becomes the contention point (i.e. there are threads that can be scheduled to run but cannot get cycles because there are other threads occupying the CPU). This causes saturation, which prevents the use of a linear model to relate the CPU usage between the instrumented and production versions of the system. CPU utilization was indeed kept below $100 \%$ as seen in Figure 13.

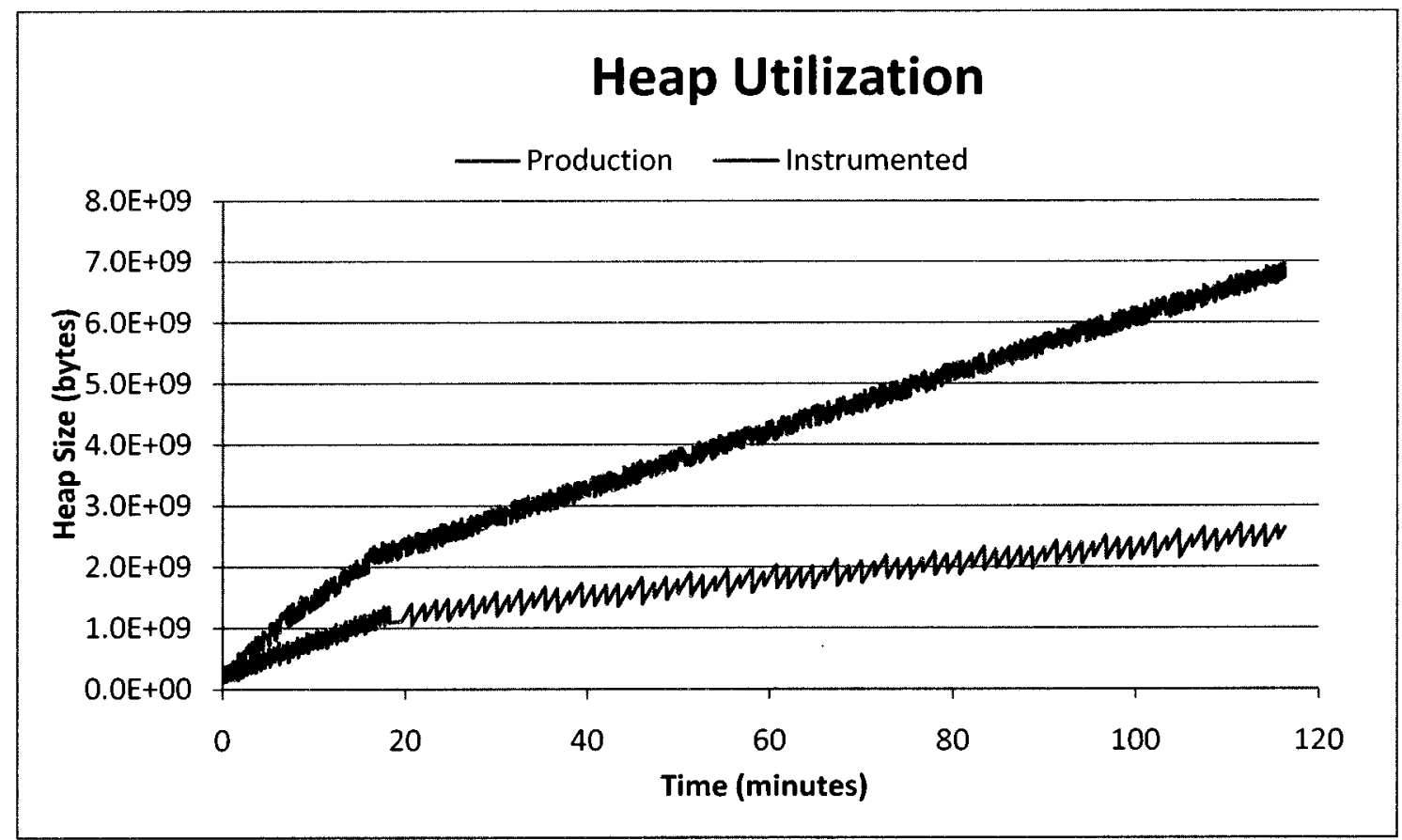

Figure 12: Comparison of the heap utilization between the production and the instrumented versions of the target system subject to the same test suite. 


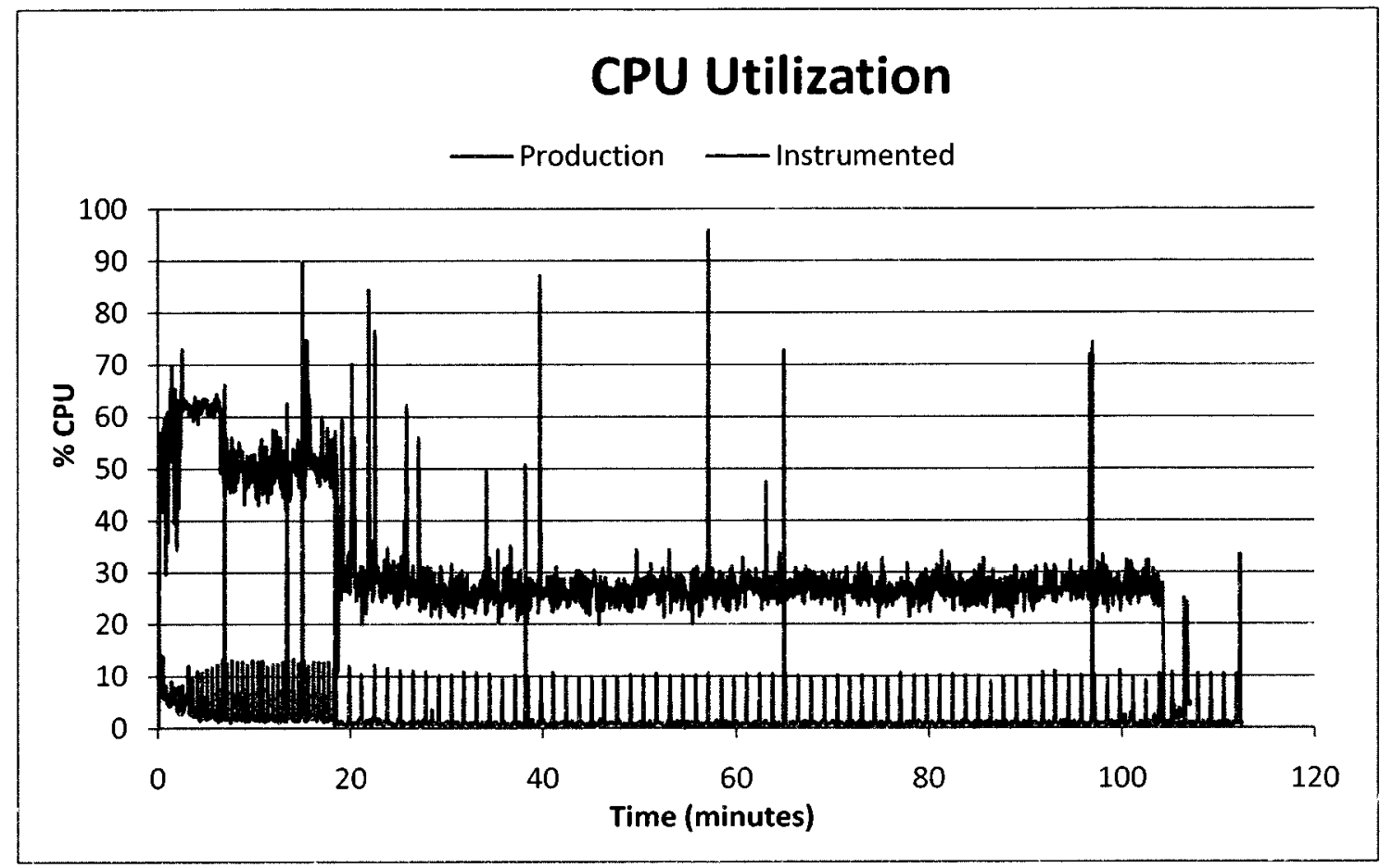

Figure 13: Comparison of the CPU utilization between the production and the instrumented versions of the target system subject to the same test suite.

The heap utilization of the instrumented version is between 2 and 2.75 times the one of the production version. There is no easy formula to determine this ratio based on static parameters as with the permanent generation size. This is because several factors like the number of instances of each class as well as the number of threads in the system ${ }^{20}$, which are essentially dynamic parameters, cannot be determined in a generic fashion for all applications since they depend on each application's structure. The important property, however, is that such ratio is bounded. Notice from the graph that although the heap size grows steadily in both versions, the ratio between them is almost constant (after the 20 minutes mark, the heap size of both versions grow linearly at a rate of $46.8 \mathrm{MB} /$ minute for the instrumented version and $13.5 \mathrm{MB} /$ minute for the production version; i.e. the instrumented version consumes heap at a constant rate of 3.47 higher than the production version), which means that the instrumented version is presenting the same behaviour as

\footnotetext{
${ }^{20}$ The checker state of an instance is stored as a thread-local object, so there is the potential for a particular instance to cause the storage of its checker instance in multiple threads. See section 4.2 for details on the JMLLocalstate nested class.
} 
the production version whilst requiring more resources. The same can be said regarding the CPU utilization.

The load factor (see section 6.1) achieved with the production version is 17.5 times the load factor achieved with the instrumented application. This means that the overall load that the instrumented version of the target system can handle is approximately 17.5 times smaller than the production system. Although this seems to be a big performance impact, one must realize that each instrumented method performs a considerable amount of work to evaluate contracts as well as manage the state in a thread-safe and non-contentious way. The amount of time spent on the generated code is of similar magnitude regardless of the method's complexity. Since the vast majority of methods in a well-designed object oriented system are small, processing speed is considerably impacted. Furthermore, in a concurrent system, the slowdown of some tasks has significantly more impact than others in case a task is holding a shared resource for which other tasks are contending. The immediate effect of this slowdown is on time-sensitive activities. Any absolute values used to detect improper behaviour like timeouts needed to be increased 20 -fold to accommodate delays in the processing due to the instrumentation overhead.

Although the biggest impact is on the amount of $\mathrm{CPU}$ required to execute the instrumented code, load parameters can be adjusted to guarantee resource usage conditions by the instrumented version similar to those of the production version. The amount of extra heap, program storage and permanent generation required by the runtime is kept at reasonable bounds thus allowing instrumented applications to be executed in the same environment used by the production version. Overall, the runtime overhead introduced by the instrumentation activity is deemed reasonable.

\subsubsection{Report 2: Indistinguishability}

The first criterion for analyzing the indistinguishability between the instrumented and the production versions of the target system is to have both versions execute the same test suite and present the same behaviour. The test suite used is exactly the same as the one used in section 6.3.3. To verify that both versions of the system behave the same in the absence of faults, contracts for the system were defined taking the behaviour presented by 
the code at a particular released version of the Service Activation Engine as correct (see 6.2.2 for details). This became the instrumented version of the system. The test suite was then executed for two hours (the standard duration) and no (contract) assertion violations were observed. To verify that both versions of the system behave the same in the presence of faults, the instrumentation was performed using a special compilation option that causes an error message to be printed on the error console (i.e. stderr on UNIX systems) instead of causing an assertion failure ${ }^{21}$. This approach allows a fault to manifest itself identically in the instrumented and the production versions of the system. All 129 faults were reproduced in both versions of the system submitted to the standard test suite.

The second criterion for analyzing indistinguishability is a qualitative analysis of the instrumentation technique to ensure that it does not prevent thread interleavings present in the production version of the system from occurring in the instrumented version. The analysis goal translates into the determination if the process of instrumenting the program with RAC code introduces any new locks, either explicitly into the generated code or by calling a method in the RAC runtime that directly or indirectly acquires one or more locks. The details of the instrumentation techniques are presented in chapter 4 . In the remainder of this section we analyze every translation function we introduced or modified to build the concurrent RAC compiler.

Safepoints cause the evaluation of pre- and post-conditions at specific points within the method, exploring existing locking patterns to ensure freedom from interference (see section 4.3 and Listing 29, p. 91, lines 8 and 9). The safepoint evaluation method is called within the already existing synchronized block. These methods simply call the assertion checking methods for pre- and postconditions, and invariants (see Listing 25, p. 83). The calls to JMLChecker.enter() and JMLChecker.exit() do not acquire any locks since the state is kept in the global checker state object (discussed below). The same is valid for commit points with respect to wait conditions. The (local) checker state necessary to

\footnotetext{
${ }^{21}$ Even though the system does not automatically exit in such cases, as it does if an assertion error is raised, the system is stopped manually since its state becomes corrupt as a consequence of an unsatisfied contract, thus invalidating any results obtained after such occurrence.
} 
correlate the pre-condition to the post-condition and to keep track if a when clause has been evaluated (Listing 25, lines 4 and 5) is kept in a thread-local object (see JMLLocalstate in Figure 9, p. 80), which does not require any extra locking to be accessed since a different instance is created for each thread. Since the assertion checking methods for preconditions, postconditions and invariants have not changed, with the exception that instance fields referenced by predicates are stored in the thread-local JMLLOcalstate object, they also do not introduce any locks. Therefore, the introduction of safepoints does not cause the introduction of locks in the instrumented program.

Locking predicates and the locks_releases clauses are evaluated in the pre- and poststates of the method in the wrapper method (see 4.4, Listing 26, p. 85, lines 1 and 18). These clauses require access to the global checker state to determine the locks acquired by the current thread as well as the lock acquisition history. Such access is performed through JMLChecker's methods hasAllLocks(), hasNoneLocks() and checkLocksClause() (see Listing 28, p. 89, lines 11 to 26 and 29 to 41). The global checker state is also kept in a thread-local object (see 4.2), thus no extra locking is required by these methods and, therefore, for the evaluation of these clauses. The same is true for the evaluation of lock ordering predicates. The only difference is that these predicates are evaluated just prior to each lock acquisition if any such clause is in effect (see 4.5 and Listing 29, 91, lines 3 to 7 and 10). All the necessary state is kept in the global checker state and thus JMLChecker's methods lockAcquired(), lockReleased(), checkInEffect(), checkLockorder() do not require locks. Therefore, no threads will block due to the instrumentation introduced for these clauses.

The evaluation of the requires_ensures_thread_safety clauses requires the use of shared state between the safe thread (the one specifying the thread-safety predicate) and potential other threads accessing the objects that are expected to be thread-safe. Locking is required to protect such shared state. Since the likelihood of a violation is very small, a data structure that minimizes the number of contentious locks to be acquired was chosen. A Concurrenthashmap is a map optimized for concurrent access. Its constructor takes the maximum number of threads expected to concurrently access it and thus creates as many segments, each with its individual lock, which is only acquired for write operations 
and almost never for reads. The keys for this map are the objects being checked for thread-safety and the values are objects containing a reference to the safe thread and an array to store any thread-safety violations observed by other threads. The map is modified only in the pre- and post-states of a method specifying a thread-safety clause (i.e. by the safe thread) to respectively add and remove a mapping (see section 4.4 and Listing 28, p. 89 , lines 2 to 10 and 27 to 28 ). This is performed through JMLChecker's methods setcheckThreadSafety() and isThreadSafe(), which do not acquire any other locks. This means that a lock will only be acquired by the safe thread. Due to the map's perthread segmented structure, the likelihood of another (safe) thread requiring access to the same segment is very small, assuming good hashing functions are used. This takes care of the safe thread. The other threads access the map via JMLChecker's methods enterMethod() and exitMethod() present in every instrumented method (see Listing 29 , p. 91, lines 1 and 12). They only perform read operations on the map to check if the object about to be accessed is owned by another thread (i.e. the safe thread). Since reads rarely acquire locks on this map, this operation will likely not cause any blocking. Therefore, the likelihood of threads blocking due to the instrumentation introduced for thread-safety is very small.

Since no significant extra blocking is introduced by the instrumentation activity, one can conclude that no interleavings present in the production version of the target system are prevented from occurring in the instrumented version. There is still the question of timing, which is fundamental to race conditions. Given the instrumentation process causes significant slowdown to tasks (see 6.3.3) there is the possibility of some race conditions to be uncovered as well as others to be hidden. The only solution for this (in the context of testing) is to let the system run for a sufficiently long time performing a sufficiently varied set of tasks, which is the same approach used for uncovering race conditions in production systems. Therefore the instrumentation does not change the testing procedure with respect to uncovering race conditions.

Given that the criteria for indistinguishability has been met, namely that the instrumented version behaves the same as the production version in the absence and presence of faults, that no thread interleavings present in the production version are prevented from 
occurring in the instrumented version, and that race conditions are uncovered by the same approach in both versions of the target system, one can conclude that both versions are indistinguishable.

\subsubsection{Summary}

This case study analyzed the ability of a concurrent system instrumented with RAC code to be used during its system test phase instead of the production version of such system. This study considered two separate dimensions of this problem, namely the runtime overhead caused by the instrumentation technique and the ability of the instrumented system in emulating the behaviour of the production system (i.e., if they are indistinguishable). Regarding the runtime overhead, although the instrumented system consumes more resources (i.e., persistent storage, memory to hold the code of classes, heap and CPU), these remain within controllable bounds provided load parameters are adjusted accordingly, which is normally easy to do in a test network if one knows the factors beforehand. Regarding the indistinguishability, the instrumented system presents the same observable behaviour as the production system since the outcome of the standard test suite to which both versions are submitted is the same in the presence and absence of faults, and that no thread interleavings present in the production system are artificially removed by the instrumentation process. Achieving success in both dimensions leads to the conclusion that an instrumented version of a concurrent system can be used during system testing to uncover faults.

\subsection{Observability and Diagnosability of Concurrency Related Faults in Industrial Software}

\subsubsection{Background on Observability and Diagnosability}

This section only presents the definitions and some basic facts on the concepts and measures of observability and diagnosability. For a complete exposition see $[36,38]$ and [38], respectively. 
The observability of a system (also called global Observability) composed of a set of interconnected components is defined as the probability that an internal fault is detected by any one of the components. The weakness of a system is the opposite probability: the probability that a fault is not detected. Let $P i(S)^{\prime}$ be the probability that a fault in component $i$, part of system $S$, is not detected in $S . P i(S)^{\prime}$ is the weakness of system $S$ on faults in $i$. Considering faults only detected by components directly connected to the faulty one $P_{i}^{\prime}(\mathrm{S})=P_{\mathrm{ij}} \Pi_{\mathrm{j}} P_{\mathrm{ij}}{ }^{\prime}$ (assuming $P_{\mathrm{ij}}{ }^{\prime}$ to be independent), where $P_{\mathrm{ij}}$ ' is the probability of not detecting a fault in $i$ by $j$. Then, the weakness of system $S$ is $P^{\prime}(\mathrm{S})=\Sigma_{\mathrm{i}}$ $P_{\text {fail }}(\mathrm{i}) * P_{i}^{\prime}(\mathrm{S})$ where $P_{\text {fail }}(\mathrm{i})$ is the probability a failure comes from executing a fault in component $i$. Let $P(S)$ be the observability of system $S$, and $P i j$ be the probability that a fault in component $i$ is detected by a direct client $j$ of $i$. The previous expression can be written as $1-\mathrm{P}(\mathrm{S})=\Sigma_{\mathrm{i}} P_{\text {fail }}(\mathrm{i}) *\left(1-P_{\mathrm{ii}}\right) * \Pi_{\mathrm{j}}\left(1-P_{\mathrm{ij}}\right)$. On the experiments in [35], the authors observed that the probability of client components to detect a fault in component $i$ could be considered approximately the same and less that the probability of detecting the fault in component $i$ itself: $P_{\mathrm{ij}}=\mathrm{c}^{*} P_{\mathrm{ii}}$, where $\mathrm{c}$ is assumed to satisfy $0 \leq \mathrm{c}<1$.

Assuming the probability of faults coming from a given component is the same for all components and that the probability of a fault in one component be discovered by its own contracts is the same for all components (i.e., $P_{0}=P_{\mathrm{ii}}=P_{\mathrm{ij}}$ for all $i, j$ ), one can conclude that $P(S)=1-\left(1-P_{0}\right)^{*}\left(1-\mathrm{c}^{*} P_{0}\right)^{\mathrm{k}}$, where $k$ is the number of clients of class $i . P(S)$ is sensitive to variations of $c$ and $k$, and therefore experiments are required to determine the global observability of a system as a function of the observability of its components $\left(P_{0}\right)$ given that $c$ and $k$ are difficult to estimate and the impact of the simplifying assumptions above is unknown [38].

Diagnosability measures the ease with which the causes of a failure can be isolated. It is measured based on an estimate of the size of the diagnosis work to be done by measuring the distance between the location of the failure detection and the location of the faulty statements that caused it. Such distance can be defined as the number of methods investigated beginning at the detection point (where the failure occurred) to the location of the faulty statement according to a diagnosis flow. 
The starting point of the diagnosis is the method in which the failure was detected. It is the caller of a method that had its precondition violated or the method that had an internal assertion or a post-condition violation. Internal assertions are not applicable to our work since contracts are completely defined in terms of pre-conditions, post-conditions and invariants, both in the functional and the concurrent facets. The search proceeds then from the beginning of the method in which the fault was detected, recursively exploring all the methods called until the fault is uncovered or the end of the method is reached. In the latter case, the search proceeds to the caller method. A method is assumed to be investigated only once. A method call is not explored if it is certain that the particular execution path leading to the fault was not executed, for instance, based on method arguments. It is explored otherwise.

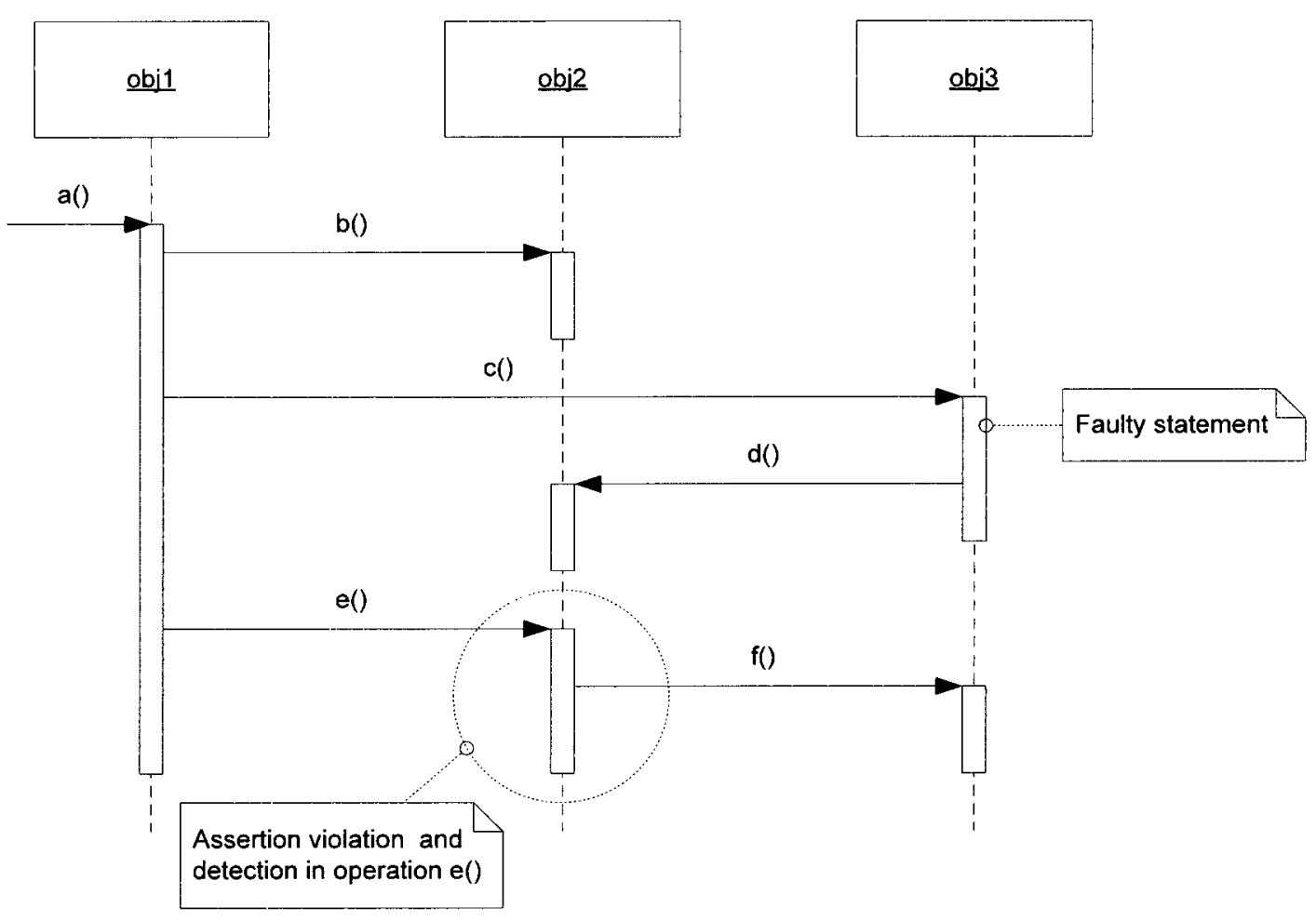

Figure 14: Diagnosability measure example: diagnosis flow as a sequence diagram.

For instance, assuming an assertion violation occurs in the precondition of method $e()$ in Figure 14, the diagnosis flow is then the sequence $[a, b, c]$ (method $d()$ is not inspected since the faulty statement is discovered in a statement preceding its invocation). The 
distance is then 3. An assertion violation occurring in the post-condition of method e () would yield the diagnosis flow [e, f, a, b, c], instead, and thus a distance of 5 .

An alternate measure of diagnosability is based on the number of statements located between the contract immediately preceding the faulty statement and the contract detecting the failure for a particular execution (i.e., the diagnosis scope) [36]. From a modelling perspective, it comprises all statements suspected of containing the fault from the failure observation point (i.e., the assertion violation) to the satisfied contract immediately preceding the faulty statement according to the specific execution thread that uncovered the failure. A good estimate of the diagnosis scope is the number of method lines of code (MLOC) for all methods included in the diagnosis scope. MLOC counts the number of non-blank and non-comment lines of code inside method bodies.

Both distance and diagnosis scope do not reflect accurately the effort a developer spends in diagnosing a failure. The distance does not take into account the complexity of the methods being investigated and the diagnosis effort does not take into account the control flow structure of the method, which is a better indication of a method complexity than simply the number of statements. McCabe's Cyclomatic Complexity (VG) measure [45] is a good indicator of the control flow complexity of a method. It basically indicates the number of execution paths a developer needs to mentally traverse to completely investigate a method for the presence of a particular fault. There are other metrics that combine control flow complexity and computational complexity [46] that are better indicators of overall code complexity or design effort. With the exception of computationally intensive software (e.g., numeric processing), which is not the target of this study, VG can help assess the effort to determine if a fault is located inside a particular method body or not. Given it does not incorporate any notion of the size of a method it can be combined with other measures related to code size.

The weighed distance measure is the number of execution paths investigated beginning at the detection point (where the failure occurred) to the location of the faulty statement according to a diagnosis flow. The diagnosis flow is identical to the one used for the distance measure. The weighed distance is then the sum of the VG measure of the 
methods of a diagnosis flow. For instance, assuming methods $a(), b(), c(), d(), e()$ and $f()$ in Figure 14 have VG of 2, 5, 3, 4, 2 and 5, respectively, then the weighed distance for diagnosis flow $[\mathrm{a}, \mathrm{b}, \mathrm{c}]$ is 10 and 17 for $[\mathrm{e}, \mathrm{f}, \mathrm{a}, \mathrm{b}, \mathrm{c}]$.

\subsubsection{Objective}

The objective of this study is to evaluate the applicability of the concurrency related constructs in contracts (chapter 3 ) as test oracles to detect and diagnose concurrency related faults. The study is limited to detecting and diagnosing race conditions and deadlocks due to the JML constructs available since it does not have constructs to specify liveness or fairness properties.

\subsubsection{Methodology}

The target system is a representative concurrent system and is specified following the methodology described in 6.2.2. The observability and diagnosability of the instrumented version of the system is measured for a series of injected concurrency related faults detected through assertion violations. The faults to be injected are retrieved from Juniper's bug database according to the following criteria:

1. It is a concurrency related fault (deadlock or race condition)

2. It is reproducible in the instrumented system

3. It was originally discovered during system testing

4. It is located in the router driver subsystem (see section 6.1) or on a directly connected client so that the failure is detected due to the erroneous behaviour of the router driver subsystem

5. It was originally discovered during a period of time in which no significant new functionality was added to the router driver subsystem.

Points 1 and 2 above are self-explanatory. Point 3 is necessary to exclude faults reported by developers during development. Such faults are discovered during coding and 
developers have the habit of filing reports to keep track of their development activities. Including such faults could artificially increase the system observability and diagnosability since they are known to be detectable a priori given the method was conceived to include such functionality and the objective of the study is to determine the use of this technique during system testing. Point 4 is necessary to limit the scope of the study to a reasonably sized subsystem to limit the effort of the case study to a reasonable level. It is not feasible to specify the complete system in the limited timeframe of this study. Furthermore, the chosen subsystem is the most concurrency intensive and displays all the concurrent behaviours present in the overall system. Faults located in directly connected clients are eligible since some locks need to (or must not) be acquired prior to executing operations in the router driver subsystem. It is expected that such faults be detected by the contracts of the methods in the interface objects. Point 5 is required so that the contracts used to specify the subsystem remain valid (i.e. they do not need to be changed) in order to inject a fault present in an earlier version of the system.

The experimental procedure is as follows:

1. Select a fault satisfying the criteria above and inject it in the instrumented and the production versions of the system

2. Run both versions of the system through the standard test suite (see section 6.1); the instrumented version should execute in a non-fatal assertion checking mode (i.e. assertion violations are simply logged instead of causing an exception to be raised).

a. If both versions of the system reproduce the fault in a similar manner, proceed to step 3. Otherwise go to step 4.

3. Run the instrumented version of the system through the standard test suite in regular mode (i.e. with assertion violations reported via thrown exceptions)

a. If an assertion violation occurs, register the occurrence and calculate the distance, weighed distance and diagnosis scope between the violated contract and the fault and go to step 4 . 
b. If an assertion violation does not occur, update the contracts, if possible, to detect the fault and go to step 3 .

c. If it is determined that the fault cannot be detected through a contract violation, record this occurrence and go to step 4 .

4. Go to the next fault and go to step 1. If there are no more faults, stop.

The decision to retrieve faults from the bug database serves two purposes: it eliminates the human factor in the fault selection process and it ensures that the faults are representative of realistic faults.

There is still the risk that such faults do not represent the complete spectrum of possible types of faults. In the absence of a fault taxonomy covering concurrency control related issues, the number or injected faults and the complexity of the system under test with respect to concurrency control (i.e. a transactional system responding to asynchronous events from devices and users with a high degree of parallelism) provide a strong argument that most types of faults occurred. Given that the system has been through multiple releases to a variety of customers and is operational in several networks supporting many different scenarios, it is reasonable to state that the vast majority of faults have already been found. This conclusion is only possible because the feature set of the system under test did not change over the period (releases) in which the faults were discovered (see point 5 of the selection criteria above).

The human factor independence enables the iteration in step $3 . \mathrm{b}$ above, thus allowing contracts to be refined in an attempt to detect an injected fault. It is an error free task to modify a contract (or a set of contracts) to detect a specific fault since the correct system in combination with the standard test suite can be used to determine the validity of the contract. The iteration approach should also minimize (if not completely eliminate) the risk of failing to modify the contract appropriately to detect a particular fault given the actual changes performed and the details on the observed failure. This will enable the determination of an upper bound in contract effectiveness. In practice the effectiveness 
will be slightly lower, depending on the developers' skills, since they will not know the correct behaviour a priori.

Success is defined by the ability of the instrumented system in detecting the injected faults and by the ease in diagnosing it. The first factor is measured by the system observability and the second by the size of the diagnosis effort in terms of the distance, weighed distance and the diagnosis scope between the fault and the contract that detected it. The higher the observability and the lower the diagnosis effort measure the more successful contracts are as test oracles.

\subsubsection{Report}

A total of 10 faults satisfied the experimental criteria defined above. All faults were detected by contracts, thus amounting 100\% observability. Table 3 summarizes the results. Race condition faults are detected by concurrency related method specification clauses (i.e. all the clauses proposed in section 3.2). Faults caused by lock ordering issues are detected by the lock_order type specification clause (see section 3.4).

Table 3: Summary of concurrency related faults observed by contracts. Faults are classified according to their nature and if contracts were updated to enable detection.

\begin{tabular}{|l|c|c|}
\cline { 2 - 3 } \multicolumn{1}{c|}{} & Contract unchanged & Contract updated \\
\hline Race condition & 6 & 0 \\
\hline Lock order & 2 & 0 \\
\hline Race condition and lock order & 1 & 1 \\
\hline
\end{tabular}

Table 3 allows us to derive two important conclusions. First that the vast majority of concurrency related faults $(80 \%)$ are related to race conditions since only $20 \%$ of them are exclusively associated with lock ordering issues. One must notice that this does not mean that deadlocks represent only $20 \%$ of concurrency related faults since deadlocks can be caused by race conditions in the evaluation of wait conditions. This only means that the effort to specify race condition related behaviour is significantly more likely to 
offer a better return in terms of fault detection than the effort spent on specifying lock ordering behaviour. The second conclusion we can draw is that concurrency related contracts written by a well-trained person are rarely incorrect or incomplete since only one fault required a contract to be updated to enable its detection. This is likely due to the simplicity of the clauses (compared to functional clauses) since one simply specifies if an object is expected to be thread-safe or if a lock is expected to be (or not to be) acquired by a thread and the pre- and post-condition of a method as well as having a separate facet dedicated for such clauses. This allows one to conclude that the likelihood of contracts written based on design information being able to detect faults is very high $(90 \%$ in this study). The small population prevents a higher precision on these results.

Regarding diagnosability, all faults have a distance measure equal to 1 , meaning that a fault is either located immediately preceding the detecting contract or in the same method that detected the fault in its post-state in such a way that no other methods needed to be investigated to determine the cause of the fault. A typical example of the former case is missing to acquire a lock via a synchronized block prior to calling the method with the detecting contract. A typical example of the latter is missing to make a method synchronized.

Despite the lack of data regarding diagnosability of concurrency related faults in the absence of contract instrumentation, it is understood such faults are difficult to diagnose. The use of contracts as test oracles clearly helps in such effort by keeping it to a minimum since the faults are located very close to the detecting contract.

\subsubsection{Summary}

The use of contracts as test oracles to detect and diagnose concurrency related faults was analyzed and deemed effective since it was able to detect all injected faults in a system that is representative of concurrent industrial software systems and such faults were located in the immediate vicinity of the contract detecting such faults. This fact is evidenced by $100 \%$ observability and by a distance measure of 1 for all injected faults. Another interesting conclusion is that the vast majority of the contracts were initially 
complete thus evidencing that the likelihood of contracts written based on design information being able to detect faults is very high.

The small population of faults considered in this study impact the precision of the results but not the general conclusions. Although one cannot reliably conclude that $100 \%$ observability would be achieved for a larger population, one can expect that the observability would be high. This also applies to the initial completeness of contracts. The lack of precision on the observability, however, does not impact the conclusion that the diagnosability of the faults observed by contracts is close to 1 given that it is a ratio measurement.

\subsection{Observability and Diagnosability of Faults as a Function of Contract Complexity in Concurrent Industrial Software}

\subsubsection{Objective}

The goal of this study is to arrive at a recommendation for the contents of contracts to be used as test oracles for concurrent industrial software. Contract content is characterized by the complexity of the constructs used to define it. Since increased complexity intuitively implies increased effort in designing a contract, this study aims at producing a recommendation on contract content in terms of the complexity of its constructs (and therefore the effort in designing it) compared to the gains in observability and diagnosability if used as test oracles in concurrent software.

\subsubsection{Methodology}

The target system is a representative concurrent system and is specified following the methodology described in 6.2.2 with one variation: to assess the effect of contract complexity on the observability and diagnosability of the system, the content of the contracts is restricted according to different levels.

Briand et al $[38,39]$ define three levels of detail of a contract for their experiment as: 
- Highest precision: every distinct condition possibly resulting from a different set of inputs or system state is distinguished in the post condition.

- Intermediate precision: only distinguishes conditions for the standard situation (expected execution) from exceptional situations that are also addressed by the method.

- Lowest precision: just defines the ranges/enumerations of values expected as resulting from executing the method.

These levels suit their work well since they consider only analysis contracts. The contracts to be developed for the router driver are at the design level. They normally include some information regarding the exceptional behaviour of a method, especially in Java since exceptions normally have to be declared in the method signature. Therefore, the intermediate precision level is not practical. Furthermore, JML provides a much richer feature set than JContract, the tool used in their work, and thus precision levels defined based on the used features of JML are better suited as a measure of the contract's complexity. The contract types used in this study are described below in ascending order of complexity (see Table 4 for a summary) and exemplified next:

- Basic: defines ranges and enumerations for native types in pre and post conditions, checks for null-ness of references and simple properties of such objects provided by their pure methods such as provided by method isEmpty () of the Java collection classes. Class invariants, if any, check similar properties of concrete fields. No model fields are allowed in any specification. Expressions using quantifiers are also not used.

- Elementary: basic plus the use of model fields, model methods, and ghost fields. Interfaces also make use of model instance fields to specify some behaviour. Model methods serve as convenience methods in this case to factor out computations that could otherwise be stated directly in the contract. They improve the contract's readability. 
- Intermediate: elementary plus quantification over elements of collections both given as arguments, data members and return values, and the use of pre-defined modeling types. The predicate of such quantified expressions should only refer to basic properties of such elements specified through the use of their pure methods or ranges of values if native types.

- Advanced: full behavioural properties are specified to the maximum detail possible. In other words, the use of specification-only pure classes (model programs [13] are not supported by the RAC [28]) designed to simulate the correct behaviour of a class or cluster of classes is allowed as well as non-trivial model methods. For our purposes, a model method is considered non-trivial if the contract specifying the behaviour of such method is required to be of type advanced to allow for complete behaviour specification (i.e. it is not just a convenience method).

Table 4: JML features allowed in contracts as a function of their type.

\begin{tabular}{|l|c|c|c|c|}
\cline { 2 - 5 } \multicolumn{1}{c|}{} & $\begin{array}{c}\text { Ranges, } \\
\text { enumerations, } \\
\text { nullness and } \\
\text { pure methods }\end{array}$ & $\begin{array}{c}\text { Model and } \\
\text { ghost fields, } \\
\text { model methods }\end{array}$ & $\begin{array}{c}\text { Quantifiers } \\
\text { and modelling } \\
\text { types }\end{array}$ & Pure classes \\
\hline Basic & $\bullet$ & $\bullet$ & & \\
\hline Elementary & $\bullet$ & $\bullet$ & $\bullet$ & \\
\hline Intermediate & $\bullet$ & $\bullet$ & $\bullet$ & $\bullet$ \\
\hline Advanced & $\bullet$ & & & \\
\hline
\end{tabular}

It should be noted that frame conditions ${ }^{22}$ can be specified by any of the contracts above and are irrelevant for this study since some violations are detected at compilation time and those that cannot be detected this way are not checked during runtime due to RAC limitations. The use of thread-safety related constructs are not subject to the above rules since they are necessary for the safe evaluation of the contracts themselves.

${ }^{22} \mathrm{JML}$ specifies frame conditions through the assignable clause (see section 2.1). 
Interface Central in Listing 31 demonstrates the differences between the types of contracts. The informal specification of method open() is translated to contracts of different types. Basic contracts are extremely easy to create since they refer only to actual fields or parameters and no quantification is allowed. The contract for method Central.open() on Listing 32 is an example of a basic contract. It refers only to parameters and specifies the exceptions acceptable to be thrown. No postconditions are specified because the return value is also an interface (see Listing 3, page 17) and model fields are not allowed in basic contracts. Notice that the contract also specifies that exception ConnectionAllocatedException can be thrown, even though it is not present in the method signature. This is because it is a child of RuntimeException, a type of exception that is not required to be present in the method signature.

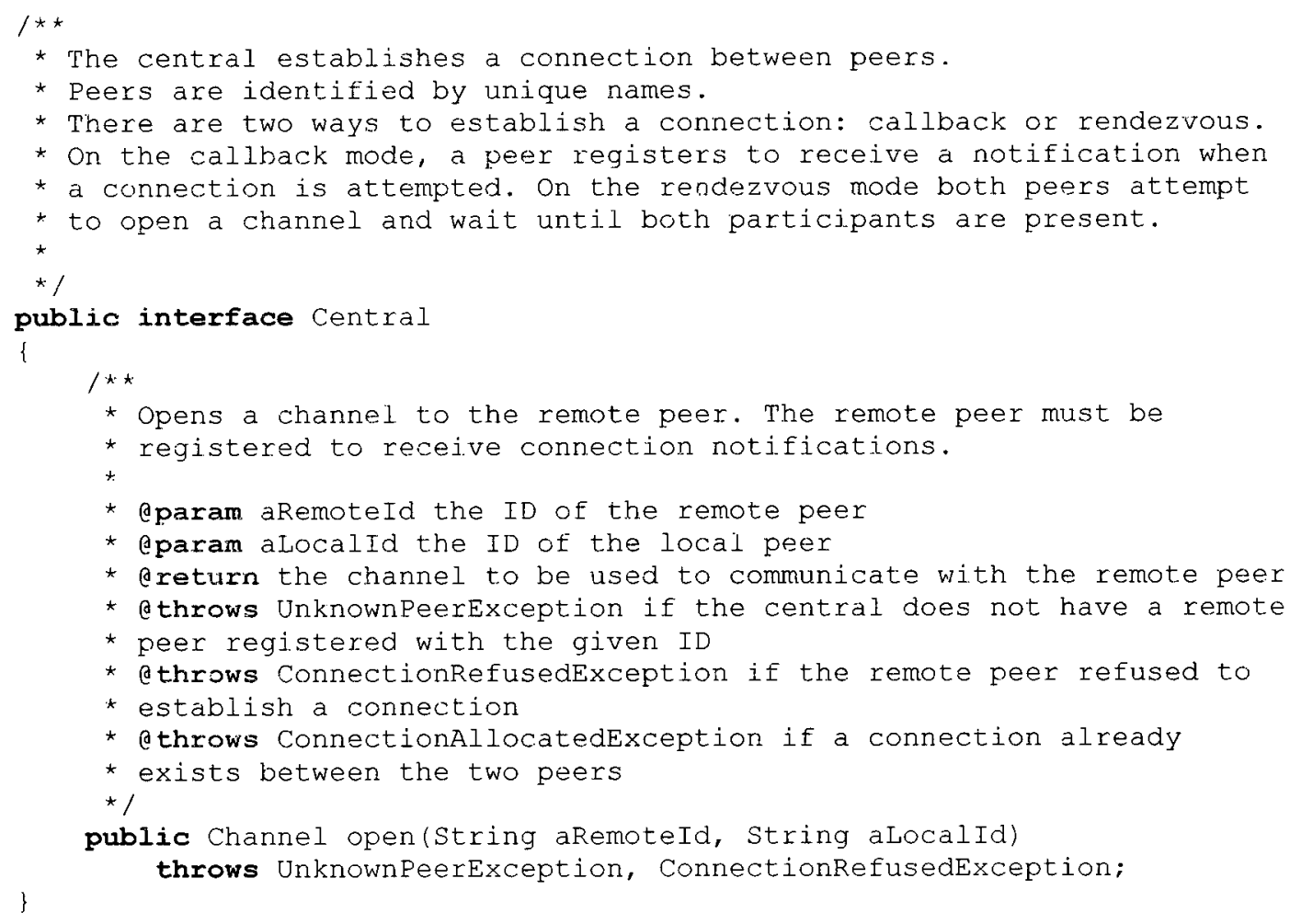

Listing 31: Interface Central to demonstrate the different types of contracts. 


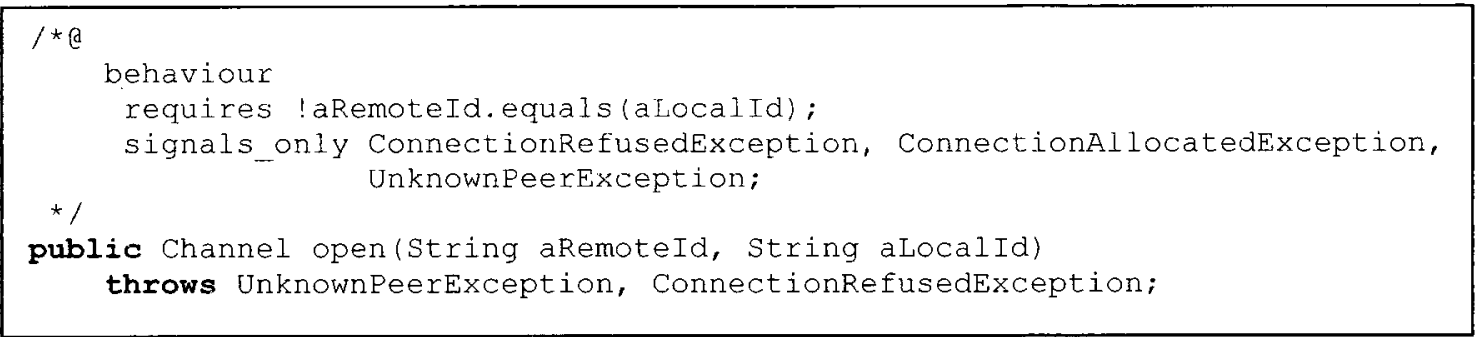

\section{Listing 32: Contract for Central .open (), an example of a contract of type basic.}

Elementary contracts incorporate the use of model fields. The added effort comes with the specification of the represents clause, the construct to map the abstract model field to a concrete implementation. Although the contract seems straightforward to state, the concrete class has to specify concrete representations for the model fields. This can become quite a complex task depending on the nature of the class. For this reason, the use of model fields is tracked as part of a separate, more complex, type of contract. Also notice that the contract can be more detailed since it can refer to model fields in the return value as well as instance fields on the interface.

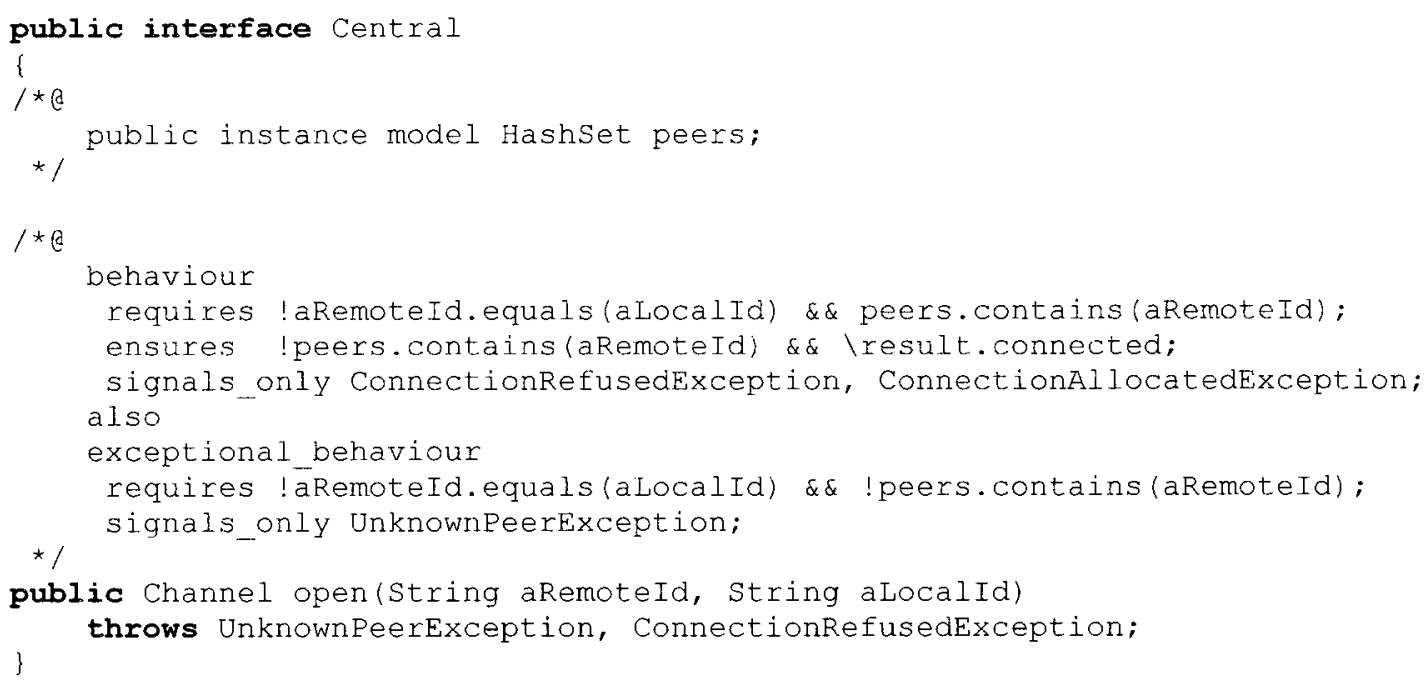

\section{Listing 33: Contract for Central open (), an example of a contract of type elementary.}

We do not present any examples of the intermediate type. The rationale for having the use of quantifiers (both existential and universal) tracked in a separate contract type is the 
added effort in specifying the expression itself, the predicate to be checked and enforcing the thread safety of all objects in the quantifier's domain. Furthermore, it is quite common to use pre-defined modelling types to conform to the purity requirements of specifications (see section 2.1). The use of such classes is also an added effort to contract design.

The advanced type of contract can be seen in Listing 34. What makes this contract of type advanced is the use of the connection class in its specification (the ghost field connections is a collection of all established connections). It is a specification-only class designed just to represent the connection between two peers. It is not actually used in the method's implementation. The added effort put in the design of such classes is considerable, which justifies tracking their use in a separate contract type. The same applies for non-trivial model methods. Notice that the complete behaviour of the method can be specified. If it was not for the use of the connection class, the invariant would be an example of a contract of type intermediate due to the use of the $\backslash$ forall (universal) quantifier. 


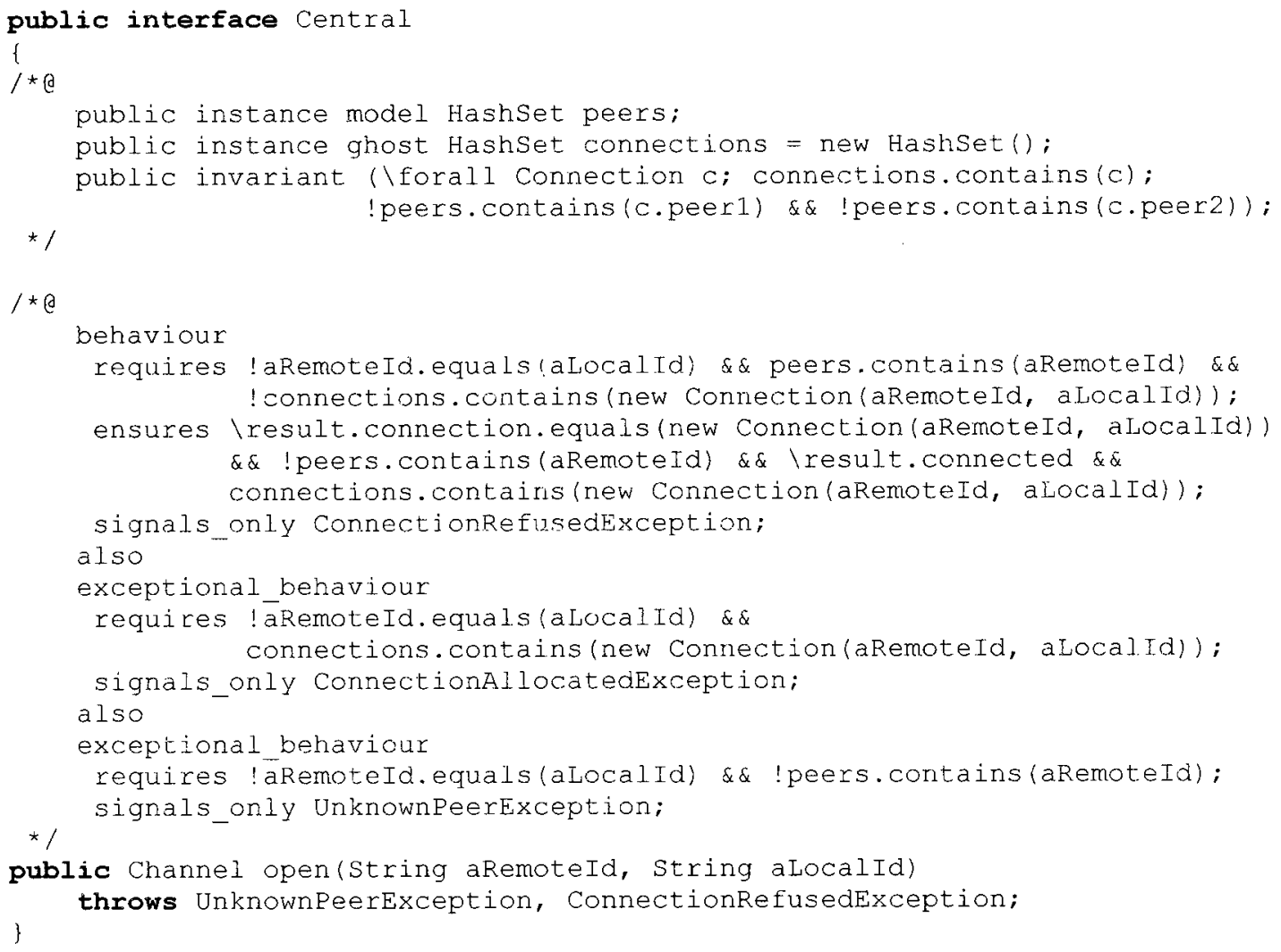

Listing 34: Contract for Central . open (), an example of a contract of type advanced.

It is clear (by construction) that contracts of a higher complexity type can specify all behaviours specified in one of a lower type. This implies that a fault detected by a contract of a lower complexity is also detected by a contract of higher complexity for the same method and type. It is also clear by looking at the preceding example that a more complex contract requires more effort to write due to the extra level of detail needed to use the more complex features of the language as well as the ability to distinguish more cases through the use of such features.

Having four different complexity levels implies that is necessary to have four different versions of the system, each with contracts of one particular type. If it is not possible for a method to have a contract of a particular type (e.g. because its behaviour can be completely specified by contract of a lower complexity), a contract of a lower complexity type will be used instead. 
Although useful, the contract type alone is not necessarily a complete indication of the complexity of a contract and, therefore, the effort in designing it. For instance, a basic contract can contain a large number of terms referring to multiple fields and method parameters while an intermediate contract can contain a single quantified expression. It is clear that, in such case, the effort necessary to design the basic contract is greater than the effort to design the intermediate contract. Another measure of contract complexity is, thus, needed to address situations like this. In the experimental procedure (to be described at the end of this section) data regarding contract complexity is collected both in terms of contract type and the new measure defined below. This enables different analysis to be conducted to correlate contract complexity to its effectiveness as a test oracle.

A measure of contract complexity (Complexity $(M)$ ) is defined according to the property-based approach for measurement definition [47]. A contract complexity measure needs to present the following intuitive properties (uppercase $M$ denotes contracts, $C$ denotes classes, $c$ denotes clauses and $s c$ denotes specification cases):

1. A contract is composed of clauses (e.g. preconditions, postconditions, invariants). Each clause can be arbitrarily complex, therefore $c \in M \Rightarrow$ Complexity $(M) \geq \operatorname{Complexity}(c)$ and $M_{1} \supseteq M_{2} \Rightarrow \operatorname{Complexity}\left(M_{1}\right) \geq$ Complexity $\left(M_{2}\right)$.

2. Clauses in different specification cases are designed independently. They only relate to each other via a specification case (see section 2.1). Grouping related clauses into specification cases increases the design effort as one needs to correlate two or more predicates: a precondition and the predicates to be satisfied as a consequence of the precondition (e.g. postcondition). Thus, $\left|M_{1}\right|_{s c} \geq\left|M_{2}\right|_{s c} \wedge \forall c\left(c \in M_{1} \Leftrightarrow c \in M_{2}\right) \Rightarrow \operatorname{Complexity}\left(M_{1}\right) \geq$ Complexity $\left(M_{2}\right)$, where $|.|_{s c}$ denotes the number of specification cases in a contract.

3. Invariants, although defined only once per class or object, are an intrinsic part of a contract and must be accounted as being both part of the precondition and the postcondition. 
4. Inherited specifications must be accounted as being part of the contract of the overriding method in the subclass. Therefore $C_{1} \leq C_{2} \Rightarrow$ Complexity $\left(M^{C_{1}}\right) \geq$ Complexity $\left(M^{C_{2}}\right)$. Where $\leq$ denotes the subtype relation between two classes and/or interfaces: the left operand is a subtype of the right operand.

5. The complexity of a clause should reflect the effort the developer spent in producing it. One way of accounting for this effort is to "measure" the number of alternatives the developer needs to consider. Therefore, $\left|c_{1}\right|_{v} \geq\left|c_{2}\right|_{V} \Rightarrow$ Complexity $\left(c_{1}\right) \geq$ Complexity $\left(c_{2}\right)$, where $\mid \cdot l_{v}$ denotes the number of disjuncts of a clause.

Following the approaches described in [47], the contract complexity measure is based on graph theory. This measure maps a contract into the control flow graph required to implement such contract in an imperative programming language. We choose Java for obvious reasons but it could be easily defined for any other imperative programming language. We use the cyclomatic complexity of the resulting control flow graph as a measure of contract complexity. A detailed translation based on the JML grammar could be easily (but laboriously) described. We present instead a simplified definition of a contract (analogous to method specifications and a subset of type specifications restricted to invariants as defined in [13]) and the resulting program. Table 5 below shows the mapping of contracts and quantifiers to the program. The mapping for clauses is a trivial mapping (except for quantifiers). The implication operators are translated into their semantically equivalents using the primitive operators available in typical programming languages ( $\varepsilon \varepsilon, 11$, and ! for Java). The equivalence operator is translated to the equality test operator $(=)$. 
Table 5: Mapping from a JML contract (top, in EBNF format) to a Java program (bottom) used to measure a contract's complexity. Mapping for universal and existential quantifiers is also shown.

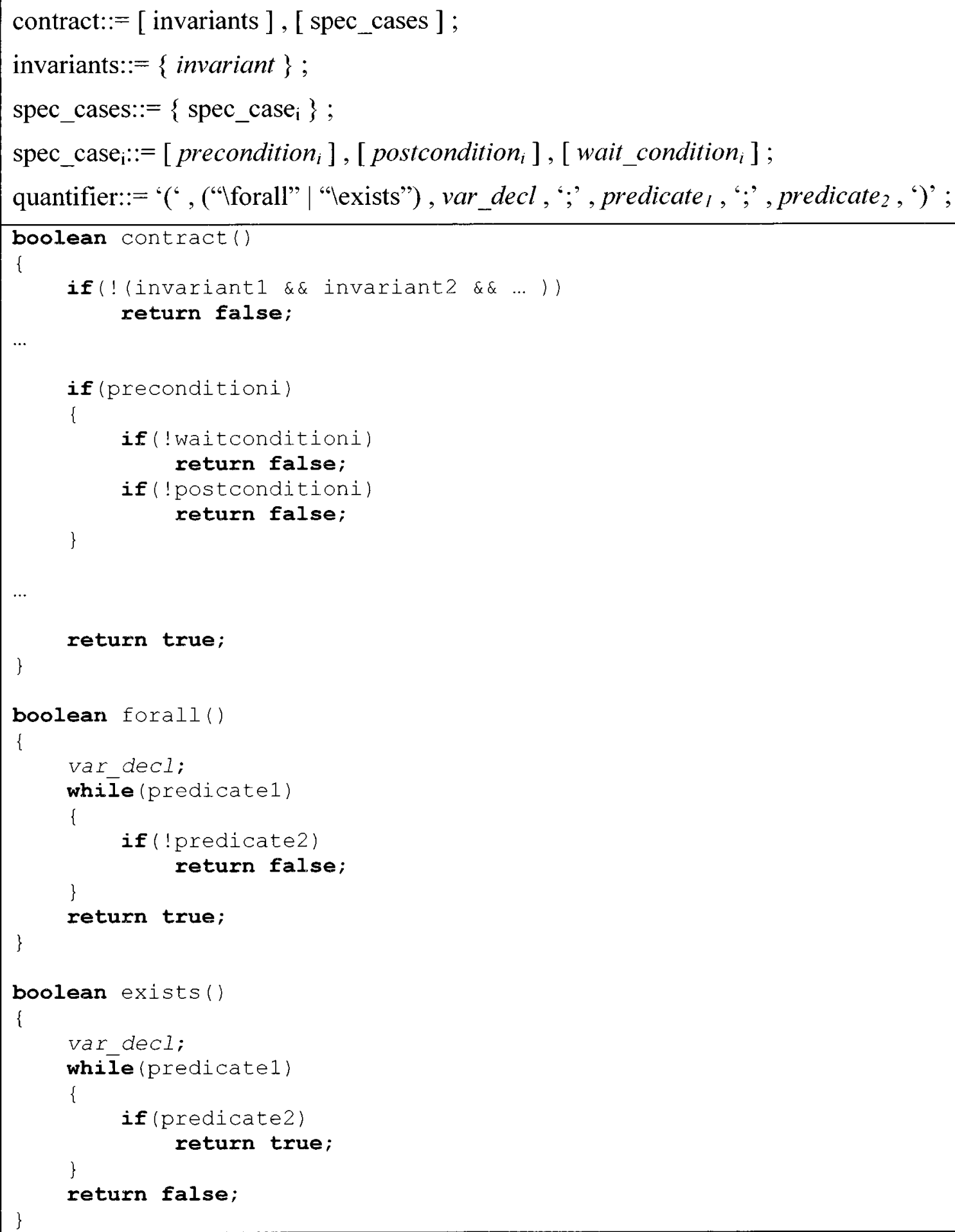


The contract complexity measure (CCM) of a contract is defined as the cyclomatic complexity (VG) of the Java program resulting from the mapping of such contract according to the rules in Table 5 minus the constant 1 . The term -1 is necessary for an empty contract to have CCM equal to zero. Model methods with bodies and methods in specification-only pure classes can be referenced from clauses. Different methods perform different computations and should be accounted according to their complexity. Therefore $\operatorname{CCM}(m)=V G(m)$, where $m$ is a model method or a method in a specification-only pure class. This translates to a very simple rule: $\operatorname{CCM}(M)=$ $|\{c \in M\}|+\sum_{c \in M}\left(|c|_{V}+|c|_{\Lambda}\right)+\sum_{\{m:(\forall c \in M)(m \in c)\}} V G(m)$, where $c$ iterates over clauses, $m$ iterates over model methods referenced in clauses, and the operators $\mid . l_{\vee}$ and $|.|_{\Lambda}$ denote the number of disjuncts and conjuncts of a clause, respectively[45] $]^{23}$. This rule can be used to compute the CCM of a contract without having to translate it to a Java program. Specification inheritance is trivially incorporated considering that inherited specifications behave as if they were defined in the overriding method (and type) [9]. For instance, the CCM of the contract of method open() of class Central in Listing 34 can be computed by following the calculation described in Table 6. It yields a CCM of 25 .

The observability and diagnosability of the instrumented version of the system is measured for a series of injected concurrency related faults detected through assertion violations. The faults to be injected are retrieved for Juniper's bug database according to the following criteria (identical to section 6.4.3 except for criterion 1, reproduced for convenience):

1. It is not a concurrency related fault (deadlock or race condition)

2. It is reproducible in the instrumented system

3. It was originally discovered during system testing

4. It is located in the router driver subsystem (see section 6.1) or on a directly connected client so that the failure is detected due to the erroneous behaviour of the router driver subsystem

\footnotetext{
${ }^{23}$ Based on the shortcut rules to compute the cyclomatic complexity of a function.
} 
5. It was originally discovered during a period of time in which the feature set of the router driver subsystem was stable.

Table 6: Example of the calculation of the CCM of Central . open (), which refers to specification-only pure class connection (not shown).

\begin{tabular}{|c|c|}
\hline Term & $\mathrm{CCM}$ \\
\hline Number of clauses & 7 \\
\hline 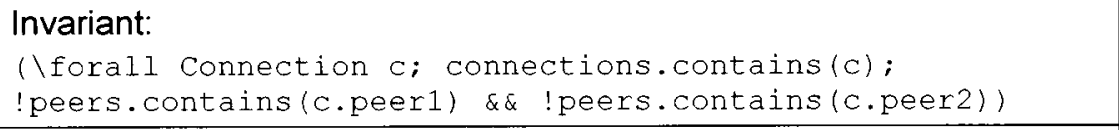 & $\begin{array}{l}3 \text { ( } 2 \text { from the quantifier }+ \\
1 \text { from the } \& \& \text { ) }\end{array}$ \\
\hline $\begin{array}{l}\text { Precondition 1: } \\
\text { ! aRemoteId. equals (aLocalId) \&\& peers.contains (aRemoteId) } \\
\text { \&\& !connections.contains (new Connection(aRemoteId, } \\
\text { aLocalId) }\end{array}$ & 2 (from the \&\&) \\
\hline $\begin{array}{l}\text { Postcondition1: } \\
\text { Iresult.connection. equals (new Connection (aRemoteId, } \\
\text { Localid) \&\& ! peers.contains (aRemoteId) \&\& } \\
\text { Iresult.connected \&\& connections. contains (new } \\
\text { Connection(aRemoteId, aLocalId)) }\end{array}$ & 3 (from the \&\&) \\
\hline $\begin{array}{l}\text { Exceptional postcondition } 1 \text { : } \\
\text { ConnectionRefusedException }\end{array}$ & $0^{24}$ \\
\hline $\begin{array}{l}\text { Precondition2: } \\
\text { !aRemoteId, equals (aLocalId) \&\& connections.contains (new } \\
\text { Connection(aRemoteId, aLocalId)) }\end{array}$ & 1 \\
\hline $\begin{array}{l}\text { Exceptional postcondition2: } \\
\text { ConnectionAllocatedException }\end{array}$ & 0 \\
\hline $\begin{array}{l}\text { Precondition3: } \\
\text { !aRemoteId.equals (aLocalId) \&\& !peers.contains (aRemoteId) }\end{array}$ & 1 \\
\hline $\begin{array}{l}\text { Exceptional postcondition3: } \\
\text { UnknownPeerException }\end{array}$ & 0 \\
\hline $\begin{array}{l}\text { Model methods are pure classes: } \\
\text { Connection methods (constructor, hashcode() and equals()) } \\
\text { referenced in the contract }\end{array}$ & $\begin{array}{l}8 \text { (6 from equals }()+ \\
1 \text { from hashcode }()+1 \\
\text { from Connection }())\end{array}$ \\
\hline
\end{tabular}

24 This clause is equivalent to signals (Exception e) e instanceof ConnectionRefusedException and does not contain a Boolean operator. Its presence is accounted for as one clause (top row). 
The experimental procedure is as follows (similar to section 6.4 .3 with a small variation to account for the different types of contracts):

1. Select a fault satisfying the criteria above and inject it in the instrumented and the production versions of the system

2. Run both versions of the system through the standard test suite (see section 6.1); the instrumented version should execute in a non-fatal assertion checking mode (i.e. assertion violations are simply logged instead of causing an exception to be raised).

a. If both versions of the system reproduce the fault in a similar manner, proceed to step 3 . Otherwise go to step 4 .

3. Run the instrumented version of the system through the standard test suite in regular mode (i.e. with assertion violations reported via thrown exceptions) beginning with the instrumented version with the lowest complexity contract type

a. If an assertion violation occurs, register the occurrence, record the type of contract that was able to detect the fault, and calculate the distance, weighed distance and diagnosis scope between the violated contract and the fault and go to step 4 .

b. If an assertion violation does not occur, update the contracts, if possible, to detect the fault and go to step 3 .

c. If it is determined that the fault cannot be detected through a contract violation, inject the fault in the instrumented version with a higher complexity contract type and go to step 3 . If there is not a version of the system with a higher contract complexity type, record this occurrence and go to step 4.

4. Go to the next fault and go to step 1. If there are no more faults, stop. 
The justifications for the fault selection and injection procedures are identical to those presented in section 6.4.3.

Success is assessed by the ability of the instrumented system in the assertions' detection rate of the injected faults and by the ease with which faults are diagnosed, respectively. If a sufficiently strong correlation between contract complexity and observability and diagnosability is observed, we will then be able to clarify the trade-off between increased effort in contract definition and increased fault detection and diagnosability. Observability is measured by the fault detection rate and diagnosability by estimating the diagnosis effort in terms of the distance, weighed distance and diagnosis scope between the fault and the contract that detected it. The higher the observability and the lower diagnosability the more successful contracts are as test oracles.

\subsubsection{Report}

We first report on observability and second on diagnosability. A total of 139 faults satisfied the experimental criteria defined above. Of these, $77.3 \%$ were detected by the instrumented version of the system through contracts of different types, as shown in Table 7. A graphical representation of this table is displayed in Figure 15.

Table 7: Faults detected based on contract type and grouped by the need to update a contract to detect it. The rightmost column shows the cumulative totals the faults of a particular contract type.

\begin{tabular}{|l|c|c|c|c|}
\cline { 2 - 5 } \multicolumn{1}{c|}{} & $\begin{array}{c}\text { Contract } \\
\text { Unchanged }\end{array}$ & $\begin{array}{c}\text { Contract } \\
\text { Updated }\end{array}$ & Total & $\begin{array}{c}\text { Total } \\
\text { Cumulative }\end{array}$ \\
\hline Basic & $31.8 \%$ & $10.6 \%$ & $12.4 \%$ & $42.4 \%$ \\
\hline Elementary & $0.0 \%$ & $10.6 \%$ & $10.6 \%$ & $53.0 \%$ \\
\hline Intermediate & $4.5 \%$ & $18.2 \%$ & $22.7 \%$ & $75.8 \%$ \\
\hline Advanced & $0.0 \%$ & $1.5 \%$ & $1.5 \%$ & $77.3 \%$ \\
\hline
\end{tabular}




\section{System Observability per Contract Types}

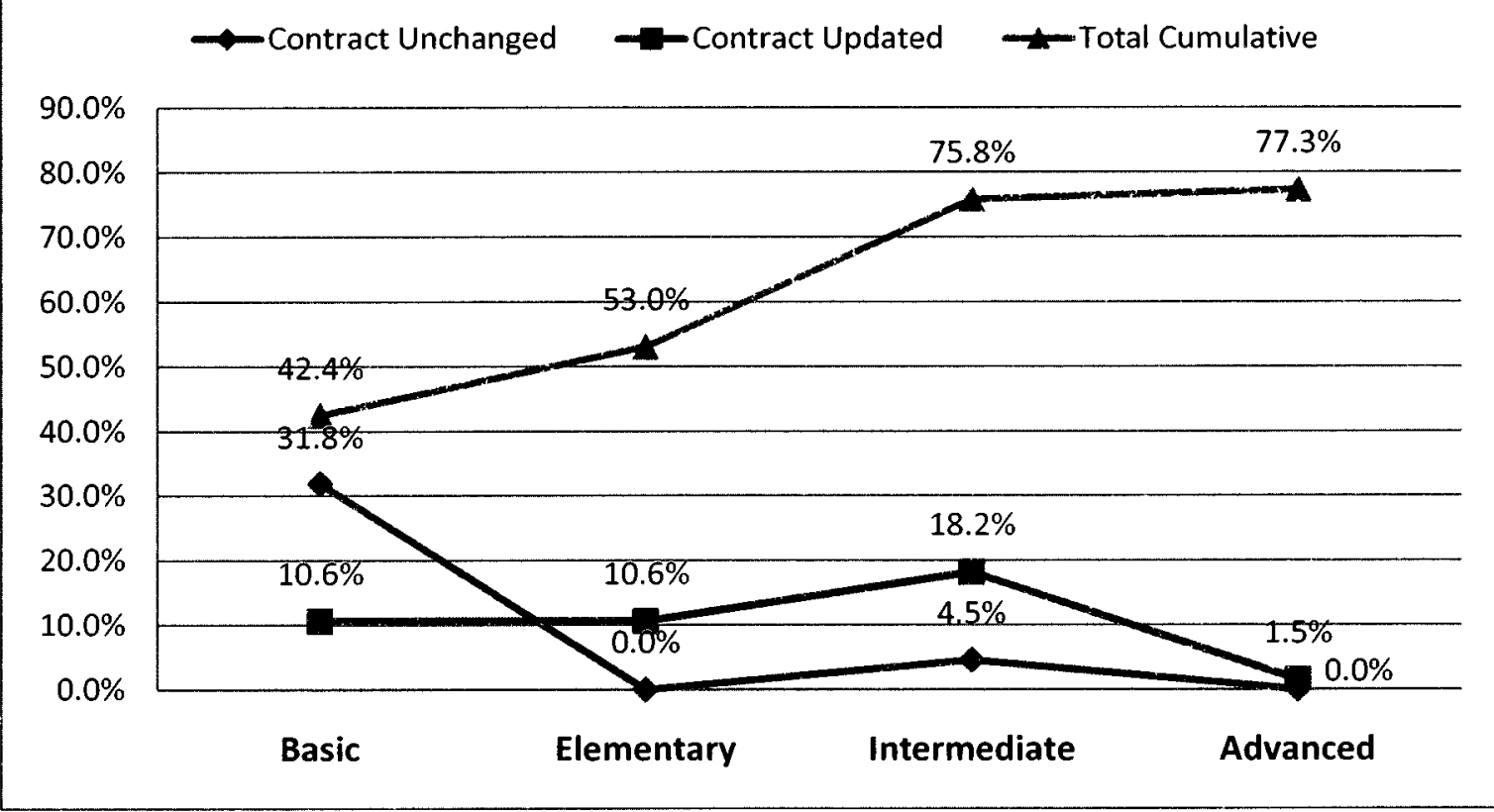

Figure 15: Graph of detected faults per contract type. The different series display the contributions of faults that required or not a contract to be updated to detect it as well as a cumulative detection rate with the increase of the contract complexity.

The results above allow us to make several comparisons between this and previous studies. The overall detection rate of $77.3 \%$ is very close to the one obtained by Briand's et al study [38] (80\%) and on the same ballpark of Baudry's et al study [35] (87.5\%). Due to the lack of detail regarding the nature of the contracts in the latter, we do not attempt to explain the difference. One important difference between this and Briand's study is that the latter does not report a significant difference in fault detection between contracts of different precisions ( $75 \%$ for the lowest and $80 \%$ for the highest), while we observe a range from $42.4 \%$ for basic contracts to $77.3 \%$ for advanced contracts. This discrepancy in the observability for contracts of different complexity can be explained by the small difference between the precision levels they describe, which is basically related to the successive inclusion of conditions to check (see section 6.5.2) whilst our categorization is based on structural constraints of the specification language (e.g. the presence or not of quantifiers). Despite such discrepancy, the same overall conclusion can be drawn: above a certain threshold it is not worthwhile to invest in contract improvements since the 
detection rate does not improve significantly. In this study, the threshold is determined by contracts of intermediate complexity since the overall observability only increases by 1.5 percentage points with the use of contracts of advanced complexity. The effort in designing an advanced contract is generally significantly higher than an intermediate one for the same method (assuming the method in question cannot be completely specified using an intermediate contract).

The previous studies mentioned above do not analyze the impact of contract updating on fault detection. Table 7 shows that $46.3 \%$ of the detected faults were found without updating contracts. This can be interpreted as the ability of developers producing contracts sufficiently complete to serve as test oracles being limited to $46.3 \%$ of the cases if no information related to faults is available to guide such effort. This interpretation is valid because contracts were designed by an expert on the system with the source code available, as described in section 6.2.2. It comes as no surprise that basic contracts were the ones that needed the least changes ( $75 \%$ of basic contracts) to detect faults since they are intuitive to design. They can only state predicates based on concrete fields and methods (as opposed to model and ghost fields and methods) and cannot state properties of elements of collections, which would require the use of quantifiers.

The elementary contracts all had to be updated, which is explained by the fact that all contracts required the use of ghost fields to model global behaviour like state machines spanning multiple classes. A typical example is the behaviour of attempting to log a subscriber in if, and only if, a default set of policies had been successfully applied on the interface terminating the traffic originating at a subscriber's equipment (see section 6.1). This behaviour is enforced implicitly by a set of classes but is not actually recorded for verification anywhere. This is the type of behaviour that is not intuitive to capture since it requires a ghost field to be set in multiple classes (in this example by the classes executing the subscriber $\log$ in) so it can be read in different contracts (located in the class controlling the policy application in this instance). Model fields did not play a role since they are a convenience to model abstract behaviour, but they do not enable the specification of any behaviour that is not possible to describe with basic contracts. 
Intermediate contracts are required to state predicates about elements of collections. The vast majority $(80 \%)$ of intermediate contracts, however, were updated. This is due to the difficulty in determining which properties are important to specify and the effort in doing so. The hardest task is to state the subset of elements to which a predicate must hold in case such predicate specifies a relation to other elements of the same or of a different set. For instance, in a transaction (which is a set of policy objects to be applied on a router), for any object of type Policylist present in a transaction, there must be at least one object of type Ruleset associated with it in the same transaction in case these objects are being installed on the device. There are many properties like this that require nested quantification (two in this example) and not all of them are relevant to detecting faults.

As pointed out in [35], contracts describe some "important" behaviours but they are not expected to be a complete description of a method. The difficulty is in determining which behaviour is important to be specified. To understand what is important to be in a contract it is necessary to first understand what it cannot detect. Table 8 summarizes the reasons for failing to detect faults. Only $6.7 \%$ of the undetectable faults are related to the constraints imposed by safepoints (see section 3.1). There is nothing one can do about it. There will always be properties that cannot be specified because of such constraints. Using an incorrect value for a timeout scores the same $6.7 \%$. This type of error cannot be detected because it is a hardcoded value that requires an agreement between two entities and any contract would just be referring to the same value the implementation refers to. Parsing errors cover $13.3 \%$ of the cases. They are undetectable because the contract would have to fully describe the actual parsing routine, which defeats the purpose since contracts should remain at a higher level of abstraction than the implementation they specify. Notice that one is not stating that it is not possible to define contracts for the parser itself (it was not done so since this functionality resides outside the router driver subsystem) but that, from a client perspective, stating the acceptable contents of a message is equivalent to writing the code that drives the parser. Missing functionality (e.g. unimplemented event handlers) accounts for $20.0 \%$ of the cases. Contracts cannot detect such faults unless an observable state change is required as a consequence of the missing statements in a method, for instance handling a particular type of message requires a subscriber to be logged out, as opposed to unobservable (or unpredictable) 
actions like handling an event notifying the system of a configuration change: a contract would have to fully describe the method to determine what to check. Internal method state (e.g. failing to check for null or to clone an object to use for certain computations) is the top ranking reason (53.3\%) for failing to detect a fault. It is impossible for contracts to detect such situations since there is no property observable in the pre- or post-state of a method that would indicate or control whether certain actions are performed. For instance, in the case of accessing a field that is allowed to be null and failing to check for its nullity inside a method is not a detectable fault since observing a null value is not an unexpected situation.

Table 8: Summary of reasons for failing to detect faults through contracts. Frequencies are relative to undetectable faults.

\begin{tabular}{|l|c|}
\hline \multicolumn{1}{|c|}{ Reason for non-detection } & Occurrence \\
\hline Incorrect timeout & $6.7 \%$ \\
\hline Internal method state & $53.3 \%$ \\
\hline Missing functionality & $20.0 \%$ \\
\hline Parsing error & $13.3 \%$ \\
\hline Safepoint constraints & $6.7 \%$ \\
\hline
\end{tabular}

We opted for a generic approach to determine the contents of a contract based on their complexity, which is measured according to the Contract Complexity Measure (CCM) defined in section 6.5.2, since we only know which types of faults cannot be detected. This approach relates a structural property of contracts (the CCM) to a structural property of methods (MacCabe's cyclomatic complexity) and the global observability such contracts achieve. This allows one to derive guidelines independently of the behaviour of a method. Instead, they serve as constraints on the complexity of a contract. 


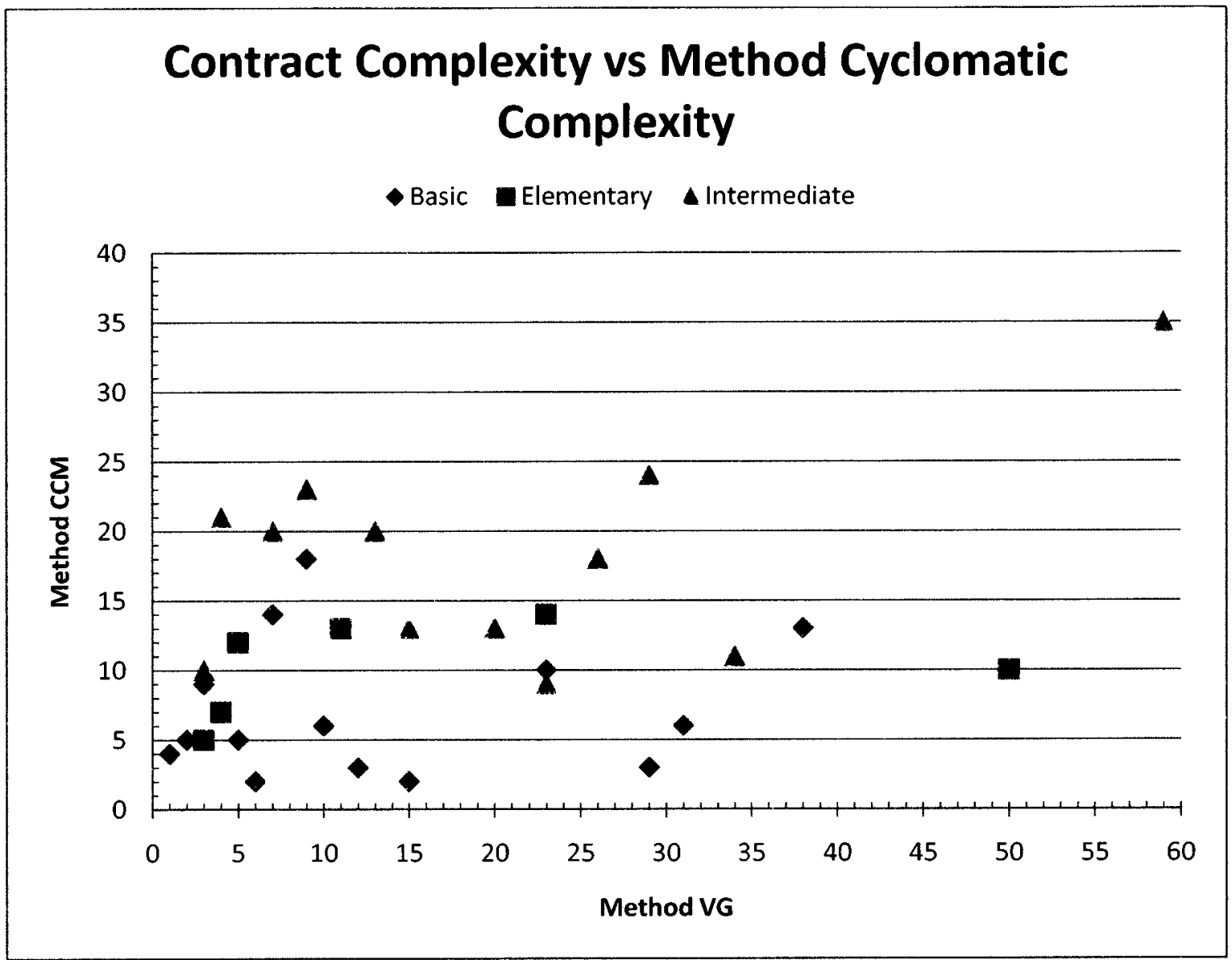

Figure 16: Graph relating the CCM of contracts to the VG of the methods they specify. Each point in the graph is the maximum value of the CCM of a contract of a particular type that detected a fault.

We first attempt to identify a relation between the CCM of a contract detecting a fault and the complexity of the method it specifies. Figure 16 depicts the relation between the cyclomatic complexity (VG) of a method and the CCM of contracts of different types. Each point represents the maximum value of the CCM for contracts of a type that detected a fault. It shows that there is no simple relation between VG and CCM. It shows only that simple methods $(\mathrm{VG} \leq 4)$ have simple contracts $(\mathrm{CCM} \leq 10)$ and that very complex methods $(\mathrm{VG}>40)$ can have very complex contracts $(\mathrm{CCM}>30)$, which is intuitive. It also shows that the vast majority of contracts have a CCM smaller than 25 , thus indicating that an analysis of fault location and detection distributions based on methods' VG and CCM could provide a good indication of the contents of a contract with 
respect to the probability of a fault being detected by the system, i.e. its global observability. Figure 17 shows the distribution of detected faults per CCM of the detecting contract. The absolute frequency values for a particular bucket are not relevant. What matters is the cumulative value of fault detection percentage, which defines a threshold on the CCM of contracts above which a certain percentage of faults in the overall system can be detected. The underlying assumption is that, for a given method, a contract with higher CCM can detect at least the faults a contract with lower CCM can. For the proper interpretation of Figure 17 one must note that contracts are a consequence of the methods they specify as opposed to the method's VG, which is an intrinsic property of each method. That is, a contract's level of detail (and therefore its CCM) is at the will of the designer and, therefore, cannot be used to classify methods in the same manner that VG is used. For instance, statistics such as fault density are meaningless if associated with the CCM of a contract.

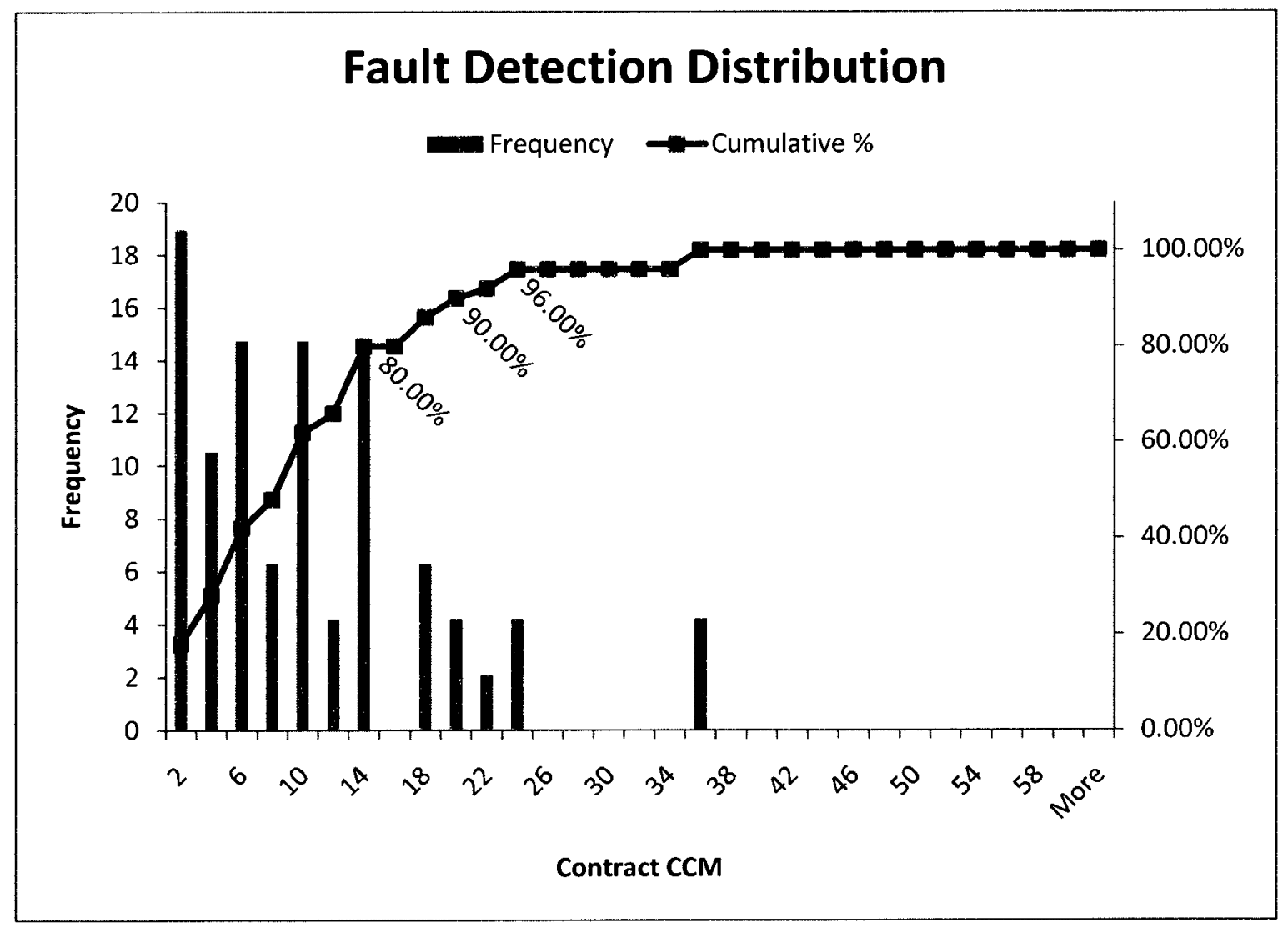

Figure 17: Histogram of faults per detecting contracts' CCM. 
The histogram in Figure 17 shows $80 \%$ of the observed faults were detected by contracts of CCM lower than or equal to 14 . Contracts of CCM up to 20 detected $90 \%$ of faults and a CCM up to 24 detected $96 \%$ of the detectable faults. Given the low precision due to the relatively small population of faults, the CCM to attain the 96 percentile could be safely chosen as 30 , the midpoint between 24 and 34, the limits of such interval. This threshold should be used as a guideline to a CCM upper-bound, i.e. when to stop specifying. It should not be used, however, as a goal for all methods since for most simple methods (i.e. those with VG $\leq 4$ ) the CCM remains below 10 (see Figure 16). It does apply, however, to most methods with $\mathrm{VG}>4$, for which contracts with $\mathrm{CCM} \geq 20$ are common, irrespective of the method's VG.

In some cases it is relatively easy to reach a CCM of 20 or higher, due to the complexity of the class, normally implying more complex invariants, which are included in the CCM of all methods in the class. In other cases, this is not a trivial effort and in others, it is not possible due to the simplicity of the class. A guideline on which methods to focus the effort should be based on the return obtained for such effort in terms of the likelihood of faults being detected by such contract. Given that the vast majority of faults are detected by the contracts of the methods where they are located (this will be discussed later during the analysis of the results on diagnosability) one can use a method's properties (in this study, its VG) to determine the likelihood that faults are present in such method and, therefore, the likelihood that its contract will detect a fault. This is depicted in Figure 18 by the fault density curve.

Despite the relatively high number of faults present in simple methods, due to their large number, it is very unlikely that simple methods are faulty, which is an intuitive result confirmed by the low fault density for methods with VG $\leq 4(0.026)$, thus yielding the guideline that contracts with $\mathrm{CCM} \leq 10$ suffice for such methods and one should not spend extra effort in refining such contracts to get close to this threshold. These represent approximately $85 \%$ of all methods in the system (see Figure A.3 in section 9.3). 


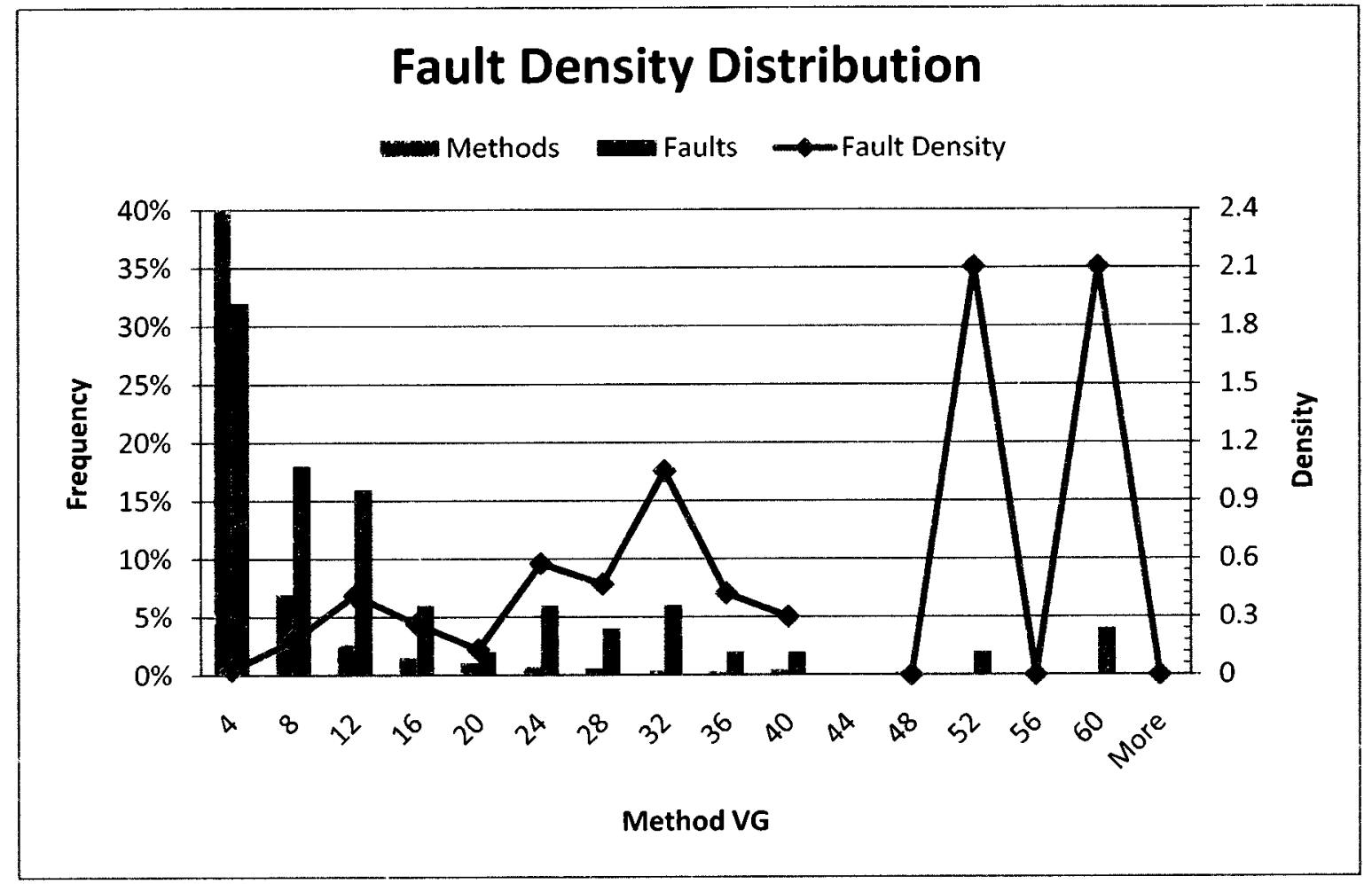

Figure 18: The fault density distribution per method cyclomatic complexity. The method frequency for $V G=4$ is $84.64 \%$.

The fault density for methods with $V G>4$ and $V G<40$ is significant thus making it the range of focus for defining contracts with CCM between 20 and 30 (depending on the desired global observability). Given the single digit method frequencies on the latter range, the shape of the fault density curve might be inflated, thus making the VG threshold determination based solely on it uncertain. This limit was chosen because it marks the 99 percentile (see Figure A.3). Methods with $V G \geq 40$ are very rare (less than $1 \%$ ), however, due to their complexity, they are very likely to be faulty and, therefore, an effort in designing complex contracts for them has a high chance of paying off and thus the CCM threshold does not apply.

The choice of the VG and CCM thresholds is ultimately based on the effort required to design the contracts, the available resources and the desired levels of observability. Figure 19 displays the CCM distributions for different types of contract. 100\% represents the total number of faults detected by contracts of a particular type. For instance, a value 
of $96 \%$ for intermediate contracts means a global observability of $73 \%$ whilst it means an observability of $41 \%$ for basic contracts (see Table 7). The line for intermediate contracts is identical to the cumulative line of Figure 17. It shows that $97.1 \%$ of faults detected by both basic and elementary contracts were found by contracts with a CCM up to 14 whilst it is necessary to have contracts with CCM up to 24 to attain $96 \%$ of detectable faults by intermediate contracts. Given that basic contracts are much easier to design than intermediate ones, one may choose to restrict contracts to be of the basic type and, therefore, defining a CCM upper bound of 14 since $97.1 \%$ of all faults are likely to be detected in such case.

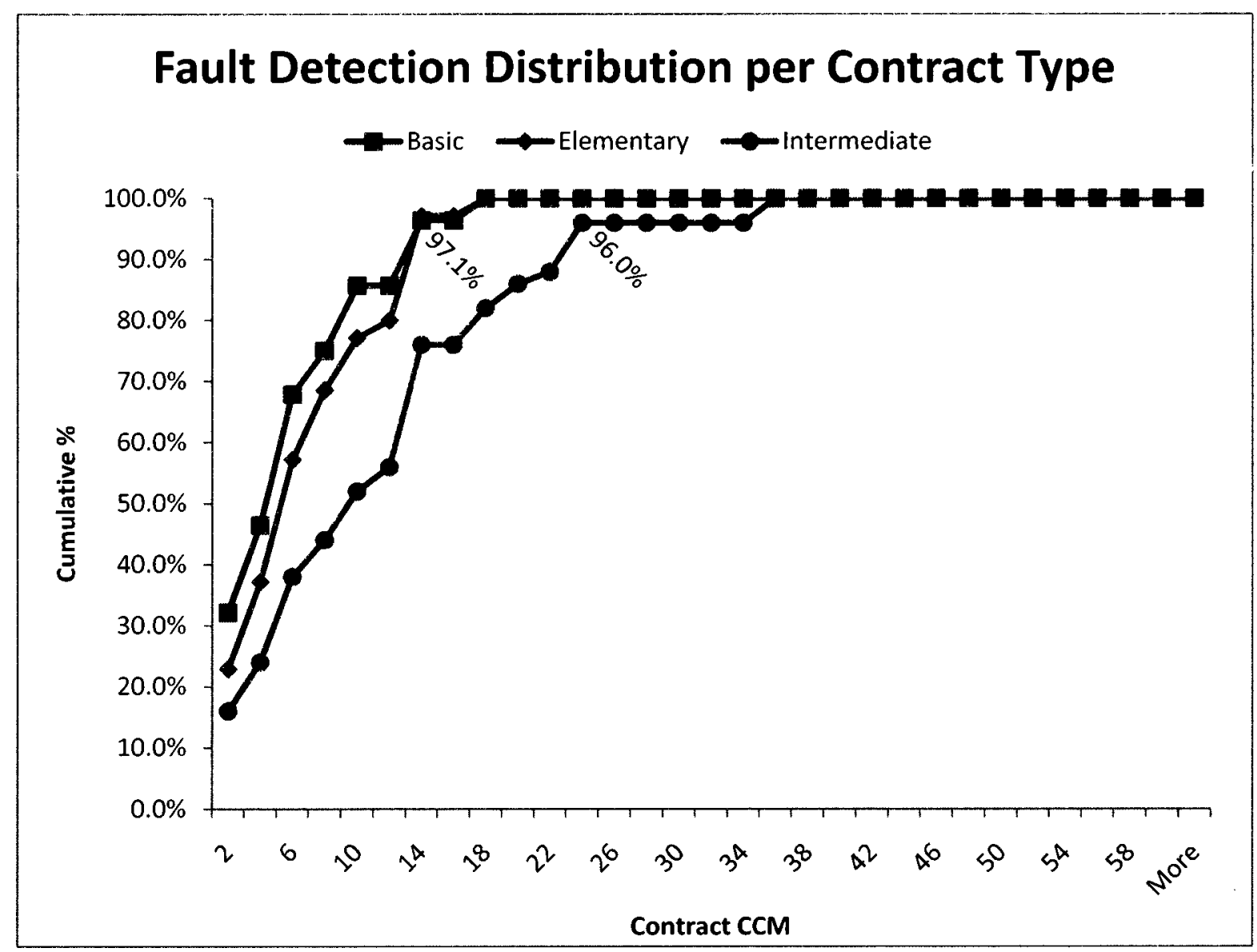

Figure 19: Cumulative distribution of faults per detecting contracts' $\mathrm{CCM}$ for different types of contracts. $100 \%$ represents the total faults detected by the given type of contract. 


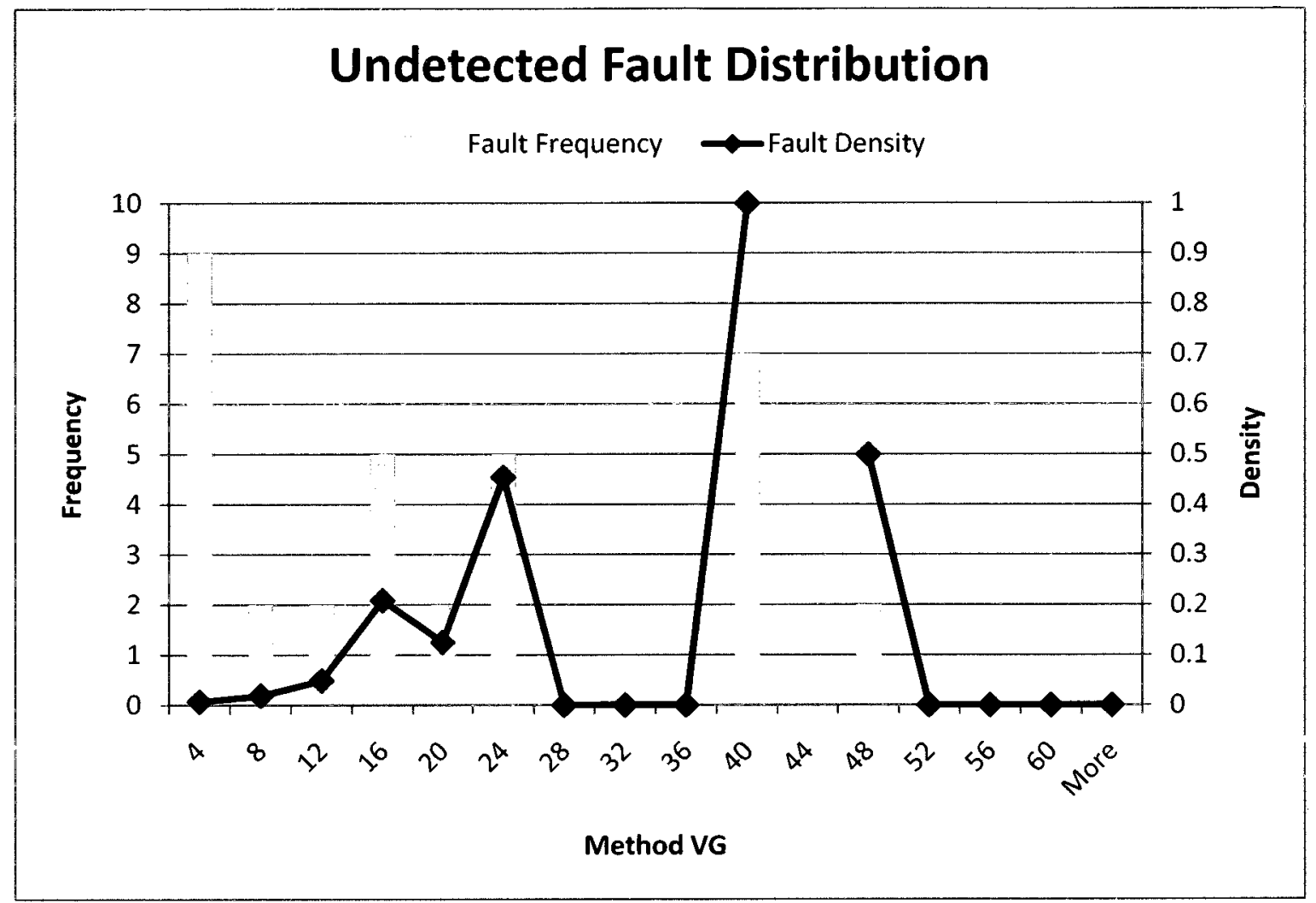

Figure 20: Histogram and density of undetected faults per method cyclomatic complexity.

Notice that undetectable faults (by contracts) are more likely to occur in methods with higher VG. This is substantiated by the histogram in Figure 20. Therefore, one should analyze such a method for the presence of the behaviours from Table 8 and design detailed manual oracles to cover such behaviours.

Any contract content recommendation should also be based on its effect on fault diagnosability. As described in section 6.5.2, we collected diagnosability information based on three different measures, namely the diagnosis scope, the distance, and the weighed distance (see section 6.4.1). These are called the diagnostic effort. These measures produce different absolute values for the same fault. To enable the comparison between them, we adopt the definition of global diagnosability presented in [36]: $\Delta=1-\frac{\delta}{\Theta}$, where $\Delta$ is the global diagnosibility, $\delta$ is the absolute measure of diagnostic effort, and $\theta$ is the maximum value of the diagnostic effort for an execution thread $T$. The 
value of $\theta$ is then the total number of statements (methods or sum of methods' VGs) traversed on an execution thread for a diagnostic scope measured by diagnosis scope (distance or weighed distance). The global diagnosability then varies between 0 , for the case one must traverse the whole execution thread to diagnose a fault (i.e. no contract detects a fault), and 1, for the (asymptotic) case the faulty statement coincides with the one generating the assertion error. To enable the easier comparison of diagnostic effort measures, we chose a reference execution thread (the one encompassing the most faults) and adjusted the measures for faults located within different execution threads. The reference thread has $\theta$ equal to 2587,147 , and 659 for the diagnosis scope, distance and weighed distance measures, respectively.

Figures Figure 21, Figure 22 and Figure 23 show the distribution of the diagnostic effort for contracts of different types according to the diagnosis scope, distance and weighed distance measures, respectively. They all have the same general shape: a peak on the left accounting for the majority of the detected faults and a peak on the right accounting for the undetected faults. The total of undetected faults is comprised of the undetectable faults (22.7\% of all faults as seen in Table 7$)$ and the faults not detected by contracts of a particular type. They are assigned the value of $\theta$ to represent the fact that they are only detected by non-contract based test oracles. Another similarity between the distributions is that although the increase in contract complexity causes an overall improvement in the diagnostic effort the incrementally detected faults are harder to detect. This is demonstrated by Table 9, Table 10 and Table 11, which display statistics on the diagnostic effort for different types of contracts according to the diagnosis scope, distance and weighed distance measures, respectively. The median and the mean decrease if the population includes the undetected faults thus demonstrating the diagnosability improvement with the increased contract complexity. Whereas they increase if the population does not include the undetected faults thus demonstrating that the incrementally detected faults are harder to diagnose. 


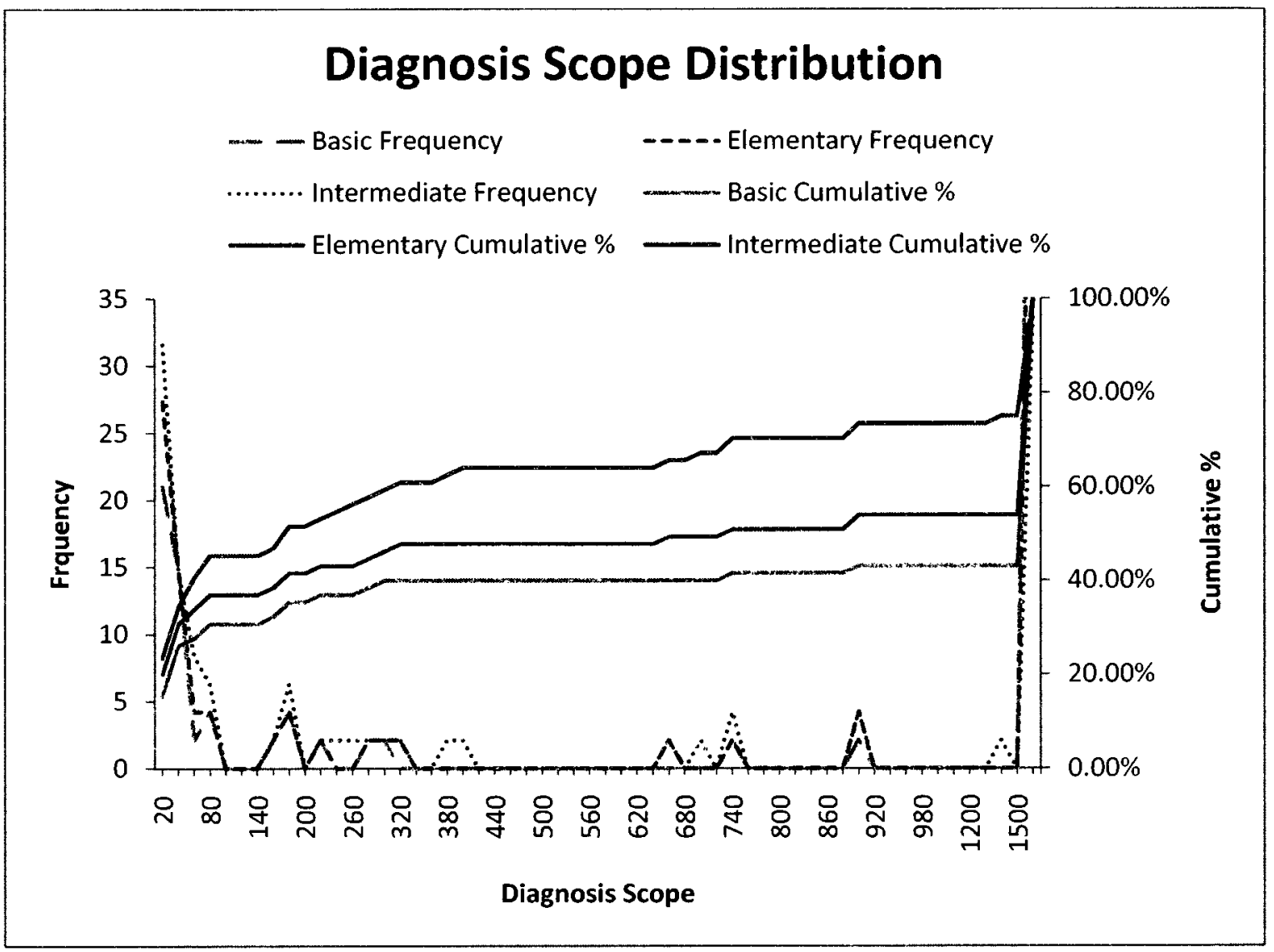

Figure 21: Histogram depicting the diagnostic effort according to the diagnosis scope measure for contracts of different types.

Table 9: Statistics for the diagnostic effort according to the diagnosis scope measure for contracts of different types.

\begin{tabular}{|c|c|c|c|c|c|}
\hline & & Mean & $\begin{array}{l}\text { Standard } \\
\text { Deviation }\end{array}$ & Median & $\begin{array}{c}\text { Avg Absolute } \\
\text { Deviation }\end{array}$ \\
\hline \multirow{3}{*}{ 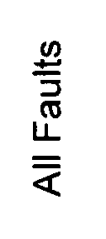 } & Basic & 1524 & 1229 & 2587 & 1063 \\
\hline & Elementary & 1276 & 1227 & 733 & 1178 \\
\hline & Intermediate & 774 & 1044 & 166 & 737 \\
\hline \multirow{3}{*}{ 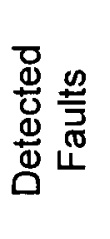 } & Basic & 120 & 210 & 29 & 106 \\
\hline & Elementary & 152 & 248 & 30 & 137 \\
\hline & Intermediate & 230 & 371 & 49 & 211 \\
\hline
\end{tabular}




\section{Distance Distribution}

$-\ldots$ Basic Frequency

........ Intermediate Frequency

- Elementary Cumulative \%
- Elementary Frequency

Basic Cumulative \%

Intermediate Cumulative \%

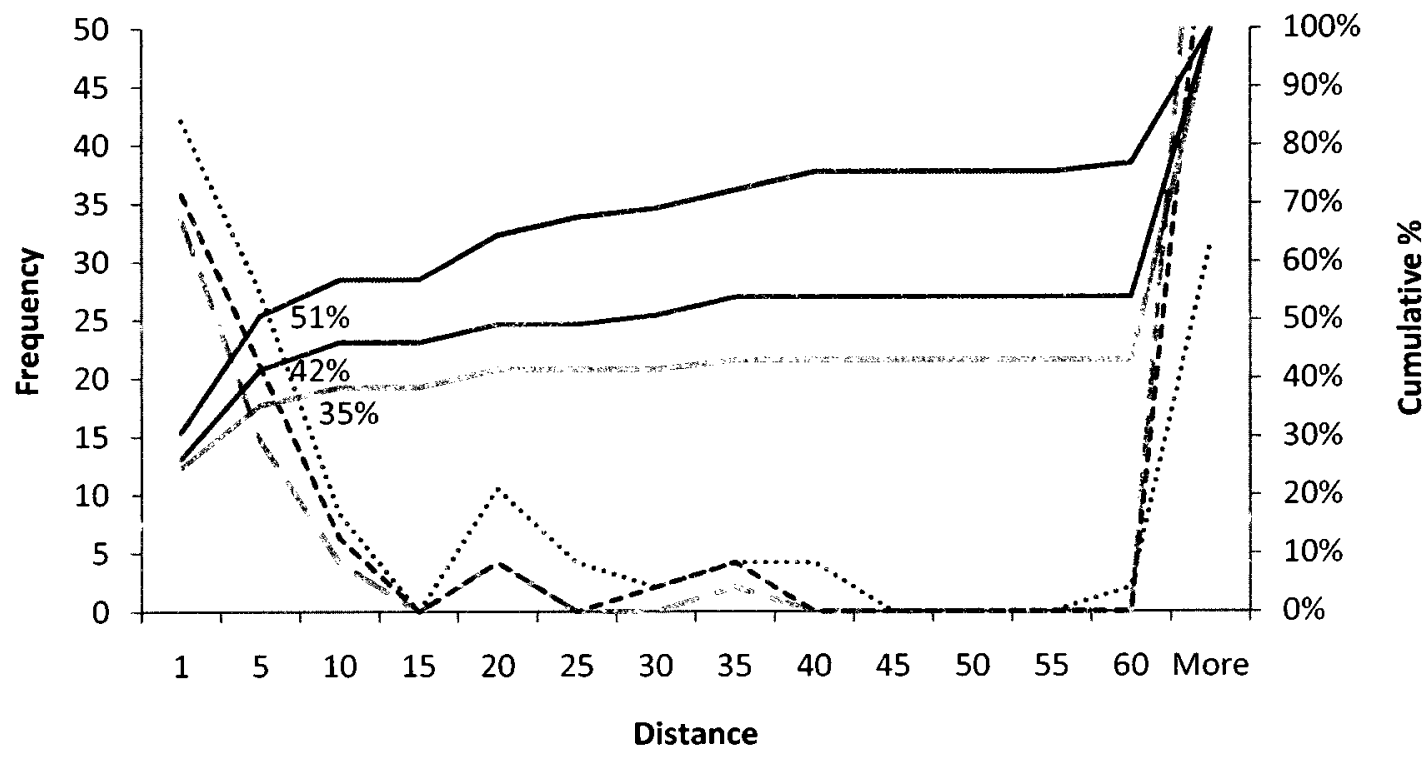

Figure 22: Histogram depicting the diagnostic effort according to the distance measure for contracts of different types. The labels shown are for the bucket 5 .

Table 10: Statistics for the diagnostic effort according to the distance measure for contracts of different types.

\begin{tabular}{|c|c|c|c|c|c|}
\hline & & Mean & $\begin{array}{l}\text { Standard } \\
\text { Deviation }\end{array}$ & Median & $\begin{array}{l}\text { Avg Absolute } \\
\text { Deviation }\end{array}$ \\
\hline \multirow{3}{*}{$\begin{array}{l}\frac{n}{5} \\
\stackrel{n}{\tilde{D}} \\
\overline{\bar{\sigma}}\end{array}$} & Basic & 86 & 71 & 147 & 61 \\
\hline & Elementary & 71 & 71 & 26 & 67 \\
\hline & Intermediate & 41 & 59 & 5 & 39 \\
\hline \multirow{3}{*}{ 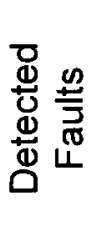 } & Basic & 4.5 & 7.1 & 1.0 & 3.5 \\
\hline & Elementary & 5.8 & 8.6 & 2.0 & 4.7 \\
\hline & Intermediate & 9 & 13 & 3.0 & 7.9 \\
\hline
\end{tabular}




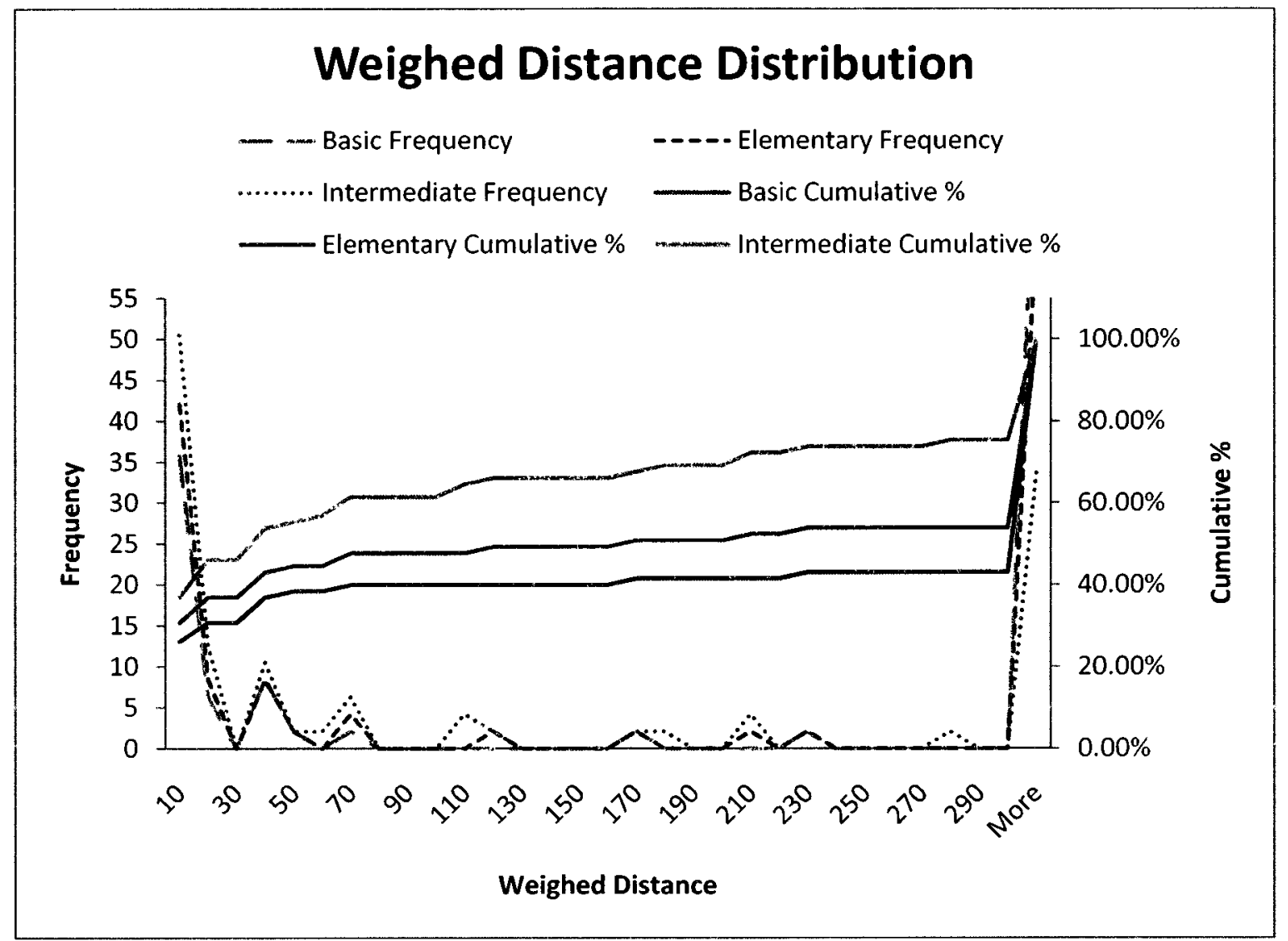

Figure 23: Histogram depicting the diagnostic effort according to the weighed distance measure for contracts of different types.

Table 11: Statistics for the diagnostic effort according to the weighed distance measure for contracts of different types.

\begin{tabular}{|c|c|c|c|c|c|}
\hline & & Mean & $\begin{array}{l}\text { Standard } \\
\text { Deviation }\end{array}$ & Median & $\begin{array}{l}\text { Avg Absolute } \\
\text { Deviation }\end{array}$ \\
\hline \multirow{3}{*}{$\begin{array}{l}\frac{0}{5} \\
\frac{\pi}{\sigma} \\
\frac{\Sigma}{\bar{\alpha}}\end{array}$} & Basic & 387 & 314 & 659 & 272 \\
\hline & Elementary & 322 & 314 & 164 & 302 \\
\hline & Intermediate & 193 & 265 & 37 & 184 \\
\hline \multirow{3}{*}{ 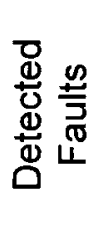 } & Basic & 28 & 50 & 9.5 & 23 \\
\hline & Elementary & 34 & 57 & 10 & 29 \\
\hline & Intermediate & 53 & 82 & 13 & 47 \\
\hline
\end{tabular}


Table 12 compares the statistics for global diagnosability obtained from the three different measures of diagnostic effort. They are virtually identical, thus indicating they are all equivalent measures of diagnosability. A Spearman correlation test reveals that the correlation coefficient between each pair of distributions is greater than 0.99 , thus confirming this finding. The table also shows the same statistics restricted to the detected faults only.

The statistical equivalence between these measures enables a choice based on the one that best represents the human effort to diagnose a fault or the one that is easier to measure. The diagnosis scope measure considers effort as the number of statements investigated. The distance measure considers effort as the number of methods investigated. The weighed distance measure considers effort as the number of control flow paths investigated. A recommendation on which one to use is beyond the scope of this study.

Table 12: Statistics for global diagnosability for contracts of different types obtained through varied measures of diagnostic effort.

\begin{tabular}{|c|c|c|c|c|c|}
\hline & & \multicolumn{2}{|c|}{ All Faults } & \multicolumn{2}{|c|}{ Detected Faults Only } \\
\hline & & Mean & $\begin{array}{l}\text { Standard } \\
\text { Deviation }\end{array}$ & Mean & $\begin{array}{l}\text { Standard } \\
\text { Deviation }\end{array}$ \\
\hline \multirow{3}{*}{ 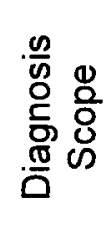 } & Basic & 0.41 & 0.52 & 0.95 & 0.92 \\
\hline & Elementary & 0.51 & 0.53 & 0.94 & 0.90 \\
\hline & Intermediate & 0.70 & 0.60 & 0.91 & 0.86 \\
\hline \multirow{3}{*}{ 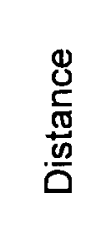 } & Basic & 0.42 & 0.52 & 0.97 & 0.95 \\
\hline & Elementary & 0.52 & 0.52 & 0.96 & 0.94 \\
\hline & Intermediate & 0.72 & 0.60 & 0.94 & 0.91 \\
\hline \multirow{3}{*}{ 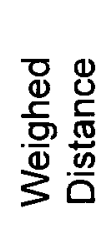 } & Basic & 0.41 & 0.52 & 0.96 & 0.92 \\
\hline & Elementary & 0.51 & 0.52 & 0.95 & 0.91 \\
\hline & Intermediate & 0.71 & 0.60 & 0.92 & 0.88 \\
\hline
\end{tabular}


Global diagnosability does not reflect one important detail: the number of faults located in the same method as the detecting contract. Table 13 displays these numbers per contract type. This means that the vast majority of faults, irrespective of the contract type, are detected by the postcondition of the method containing the fault. This aligns with the results from Briand's study [38], which observed the same phenomenon. Further comparisons of the numerical results is impossible since they used a variant of the distance measure that is not equivalent to the one used in this work.

Table 13: Percentage of faults located in the same method as the detecting contract.

The first column considers only the detected faults for a particular contract type whilst the second considers all the faults, including the undetected ones.

\begin{tabular}{|l|c|c|}
\cline { 2 - 3 } \multicolumn{1}{c|}{} & Detected Only & All Faults \\
\hline Basic & $82 \%$ & $35 \%$ \\
\hline Elementary & $77 \%$ & $41 \%$ \\
\hline Intermediate & $76 \%$ & $58 \%$ \\
\hline
\end{tabular}

As with observability, the diagnosability improves significantly with the successive increment in contract complexity (see Figure 24), thus allowing one to establish a cause and effect relation between observability and diagnosability and, therefore, to estimate the gains in diagnosability based on the effort spent on contract design. This aligns with the model in [36], which concludes that the global diagnosability is very sensitive to the contract's efficiency (the probability of a contract detecting a fault located in the statements situated between the detecting contract and immediately preceding contract in an execution thread). This is, however, the only aspect that aligns with it. The model was not able to produce values of diagnosability reasonably compatible with those obtained from experimentation. Assuming the model is correct, which stands to reason, there are only two possibilities: either the simplifying assumptions are invalid or the parameter estimation procedure is incorrect. The authors present and justify the following assumptions: 
1. The contract distribution is uniform across an execution thread.

2. The closer a contract is to a fault, the higher its probability of detecting a fault.

3. Contracts have an equal probability of detecting a fault coming from the statements immediately preceding them given they were written with such purpose.

4. Each statement has the same probability of being faulty.

Assumption 1 has been verified experimentally by the authors as well as a part of this work. Assumption 3 is true since the percentage of faults with a distance up to 5 (see Figure 22) matches the results in Table 13. The value 5 for distance is based on the ratio average number of statements in a faulty method (58.2) / contract density (17.6), which yields 3.31 contracts per faulty method, which falls into the 1 to 5 buckets. Assumption 4 is reasonable to build a model. We believe that Assumption 2, although intuitive, is false. This is evidenced by the fact that the contracts with highest probability to detect a fault are the ones associated with the faulty method and that more than 3 contracts on average are traversed in a method execution. It is unknown if this assumption is only violated in this system in particular or it is a general property of concurrent systems. Or even that it is due to the characteristic method complexity distribution of this system in particular given that the literature reports on typical object-oriented systems having small methods. Although the reasons for such behaviour are unknown, assumption 2 cannot be considered valid for all systems.

Given the uncertainty regarding the correctness of the model presented by Le Traon et al, the only conclusion pertaining to the dependency between diagnosability and observability that can be drawn is that there seems to be a linear relation between them instead of a more complex one as depicted in Le Traon's model. Although this is what has been observed for this system, there is insufficient data to generalize it, even if restricted to concurrent systems. 


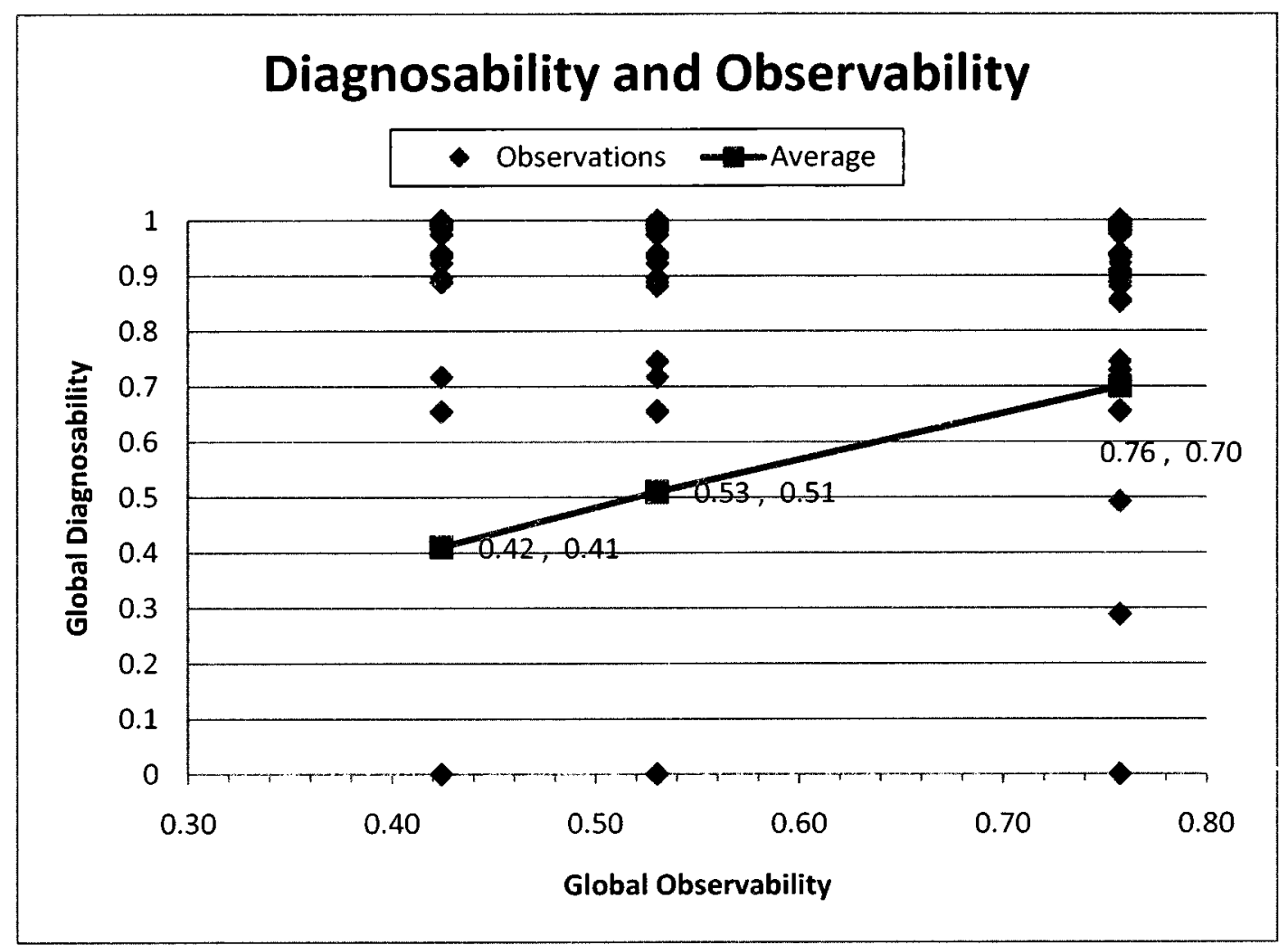

Figure 24: Global diagnosability as a function of global observability.

\subsubsection{Discussion and Summary}

This case study arrived at the following conclusions:

1. Overall, observability increases significantly with the increase of contract complexity.

2. The use of advanced contracts (those containing specially crafted classes to model abstract behaviour) does not increase the observability significantly if compared to intermediate contracts.

3. Observability is limited to about $76 \%$ if contracts are restricted to the intermediate type, whereas it is limited to about $42 \%$ for basic contracts.

4. There is no simple relation between a method's VG and a contract's CCM, therefore contracts must be treated in aggregate form independently from the method they specify. 
5. A system with contracts with CCM 20 and 30 can detect $90 \%$ and $96 \%$, respectively, of all faults.

6. Contracts with $\mathrm{CCM} \leq 10$ suffice to specify methods with $\mathrm{VG} \leq 4$.

7. Methods with VG in the range $] 4,40[$ are the ones that benefit the most from contracts with CCM between 20 and 30 .

8. Methods with $V G \geq 40$ are rare and likely faulty thus offering a high chance of payoff to contracts and, therefore, should not be restricted to having contracts with a given CCM. These methods are also the ones containing most of the undetectable faults by contracts and, thus, require the use of manual oracles to detect faults due to incorrect timeout, internal method state, missing functionality, parsing error and safepoint constraints faults.

9. The three measures of diagnostic effort presented are statistically equivalent.

10. Global diagnosability has a linear relation to the global observability, allowing one to determine the effort in diagnosing faults as a function of the targeted observability, which is a function of the contract complexity.

Based on these conclusions, a contract to be used as a test oracle in a concurrent system should possess the following properties to be both effective and efficient (not unnecessarily complex):

A. Be of type intermediate if required to specify the method's behaviour but never of type advanced.

B. Have a $\mathrm{CCM} \approx 10$ if the method has a $\mathrm{VG} \leq 4$. There are cases in which invariants are complex due to other methods' complexity. In such cases, this property can be overlooked and the recommendation is to focus on properties unrelated to invariants such as predicates involving parameters and return values. Simply not providing a contract for a method in this case will certainly let faults related to parameters and return values go undetected. In cases in which this is clearly impossible (e.g. an accessor method in a very simple class), this requirement can be relaxed.

C. Have a $\mathrm{CCM} \approx 30$ for methods of $\mathrm{VG}>4$ and $\mathrm{VG} \leq 40$. This threshold targets an observability around $75 \%$ and thus a diagnosability of around 0.70 . This means 
that it will reduce the diagnosis effort to only $30 \%$. More importantly, however, is that in the worst case (i.e. a complex fault only detectable by an intermediate contract) the average effort in diagnosing such fault will be less than $9 \%$ of the execution thread (a diagnosability of $91 \%$ from Table 12 ).

D. For methods performing tasks that are inherently prone to undetectable faults such as message parsing, time dependant actions, or that manipulate a lot of state kept in the context of the method (i.e. local variables), their contracts can have a larger CCM than mandated by the requirements above whilst having manual oracles focusing on the undetectable behaviours mentioned above.

The guidelines presented above are clear and objective. To our knowledge, there is no similar contribution in the literature based on experimental data collected on industrial concurrent systems. The CCM and VG thresholds are chosen based on the availability of resources to design the contracts and the desired global observability and diagnosability. The graph in Figure 19 can be used to relate the desired observability to the CCM threshold. The VG upper limit (40 in this analysis) should be chosen based on actual data collected for the target system (a method distribution per VG as in Figure A.3). The CCM guidelines are deemed generic based on the assumption that the target system is representative of an industrial concurrent system. A similar study should be performed to determine if such guidelines are applicable to purely sequential software.

Even if one follows these guidelines, there is a chance that detectable faults will still be present. This is evidenced by the significant number of contracts (about 53\%) that required improvement to detect faults (see Table 7), although given that the guidelines were based on data collected after a contract was updated, it is expected that the need to update contracts will be significantly less. This is because, as already mentioned, it is difficult to determine which properties should be specified and which should not. In similar manner to standard methodologies in which test cases (and test oracles implicitly) are updated as new faults are discovered, contracts should also be updated. This will increase the system's global observability and, consequently, its global diagnosability. More research is required to evaluate the continued use (i.e. across multiple evolving 
releases) of contracts as test oracles in industrial systems to identify the gains in comparison to current methods based on manually coded test oracles.

The biggest threat to the generality of these recommendations is if the structural properties of the chosen system are typical of the class of concurrent industrial systems. The method cyclomatic complexity distribution is expected to vary across systems. The effect of such variation is unknown but it is expected that the recommended $V G$ thresholds above would need to be adjusted. This would likely affect the upper end (40 in this case) since those are the methods with the smallest frequencies and thus the most sensitive to variations in the distribution. The CCM thresholds should, however, be less sensitive to such changes since even simple contracts can detect faults in highly complex methods. For instance, based on Figure 17, one can see that all detectable faults are observed by contracts with $\mathrm{CCM} \leq 38$, even though there are methods with $\mathrm{VG}=60$ (Figure 16) with bugs that were detected by contracts with $\mathrm{CCM}=35$. Therefore, the CCM thresholds should be applicable to other systems provided their method complexity distribution does not deviate too greatly from the one presented by the target system.

\subsection{Summary}

This chapter presented four case studies with the aim of determining the effectiveness of using contracts as test oracles applied to industrial concurrent systems. We first demonstrated that the constructs we proposed are essential to the complete specification of concurrency related properties: the constructs are both required to be used and capable of specifying behaviour in realistic systems.

We then showed through experimental results that an industrial system can indeed be instrumented with runtime assertion checking code and used for to support system testing: the instrumented version of the system presents the same behaviour as the production (non-instrumented) version modulo performance. We also presented detailed data on system resource consumption for both versions of the system. These can be used to tune the test bed load parameters to subject the instrumented version of the system to similar conditions as those of the production system. 
The third case study demonstrated that deadlocks and race conditions can not only be detected by such contracts but they are very easy to diagnose (the fault is located very close to the contract that triggers a failure). This is a result of high practical importance given that such faults are normally very difficult to diagnose.

Finally, the last case study determines a set of requirements contracts should fulfill to be effective test oracles. These are objective guidelines based on structural properties of both the contract (its complexity measured through the CCM, a measure we propose) and the method being specified (its cyclomatic complexity), as well as objective measures of such effectiveness, namely the ability of contracts detecting such faults (the global observability) and the associated effort in diagnosing such faults (the global diagnosability).

Each case study reported specific threats to the validity and generality of the derived conclusions. One threat common to all studies above is if the chosen system is representative of industrial concurrent systems. Based on its behavioural characteristics, there is a high degree of confidence that it possesses all the concurrency control related behaviours of typical concurrent systems. 


\section{CONCLUSION}

Different solutions to the problem of applying Design by Contract to concurrent software have been proposed. A complete solution requires the combination of specifying the behaviour of interest in the form of contracts in a suitable language, translating such contracts into runtime assertion checking code and embedding it into the program being specified (a process called instrumentation). One important use of executable contracts is to serve as automated test oracles. For any results obtained with the instrumented system to apply to the original one, both systems must be equivalent, i.e. the runtime assertion checking code must not produce any side-effects that would cause faults present in one system not to manifest in the other. Contracts are also required to be effective in detecting concurrent faults such as deadlocks and race conditions as well as functional faults in a concurrent environment.

This thesis addresses the above requirements and thus enables support for $\mathrm{DbC}$ to concurrent software. The next section summarizes the contributions to the state of the art. The final section presents possibilities of future research on this and related matters.

\subsection{Summary of Contributions}

Applying Design by Contract to concurrent software poses several challenges, namely interference, locking properties specification and thread-safety requirements specification. We tackle interference with the introduction of safepoints, which are explicit points in the method body at which it is safe to evaluate preconditions, postconditions and invariants. We define the syntax and semantics of safepoints in the context of a concurrent method. Based on this concept, we also derive the minimum thread-safety requirements for a method to be interference-free.

Our approach has a limitation, though. Not every sequential contract can be expressed as a correct concurrent contract. This is due to the inherent non-determinism of concurrent systems. This usually happens for contracts that state properties related to sizes of shared data structures. For instance, it is not always possible to guarantee that after taking an 
element from a concurrently modified queue it will contain one element less than it had prior to executing this operation. This is a limitation of the (method) implementation, not the technique, since one can trivially eliminate all concurrency control issues by externally acquiring all necessary locks prior to executing a method to guarantee its sequential execution. With such limitation in mind, however, one can design concurrent contracts that are correct and representative of the actual behaviour of an object in a concurrent environment, a fundamental problem that remained open until now.

The current approaches to the specification of thread-safety properties do not contemplate the impact of specification inheritance since they are placed together with pre- and postconditions thus being subject to the rules of some kind of specification inheritance as for functional properties. We showed this leads to unexpected behaviour. We identify and solve this problem by creating method specification clauses for thread-safety and locking requirements analogous to pre- and postconditions with different inheritance rules. We advocate that such clauses can only be strengthened by specification inheritance, meaning that the resulting clause is the conjunction of all inherited clauses of the same type, effectively treating them in the same way as invariants. With this approach one can safely specify this type of properties on interfaces, constraining their implementation to follow them but not preventing the implementation from requiring and ensuring stronger properties. We distinguish the concurrent facet of a contract, which houses clauses to specify thread-safety properties, from the functional facet thus maintaining the usual notions of behavioural subtyping for the specification of functional properties.

We introduce a special construct to the specification of a type, namely the lock_order clause, to tackle the issue of lock ordering specification. Its unique semantics solves previously reported issues with soundness of more general approaches. We feel that, although less flexible than other approaches, our solution addresses the common needs of lock acquisition ordering specification with a simple and sound semantics that avoids the use of quantifiers and logical expressions.

We validated these constructs with a case study consisting of an industry-strength concurrent subsystem. It demonstrated that the proposed constructs are not only essential 
to the proper specification of concurrent programs but that they are also capable of specifying most behaviour. The use of safepoints to specify functional properties of a concurrent system proved to be not only essential but also applicable to the vast majority of cases. We identified some limitations but we were nevertheless able to specify complex behaviours, both functional and concurrent, that could not be specified with the current JML constructs. This first set of results was publicized in "Araujo, W. L., Briand, L. C., Labiche, Y. Concurrent Contracts for Java in JML. In Proceedings of the 19th International Symposium on Software Reliability Engineering, pp. 37-46, IEEE Computer Society, 2008."

We implemented our proposed constructs on the JML toolset, including the Runtime Assertion Checker, thus confirming they are realizable. We applied the RAC to an industrial concurrent system and were able to demonstrate its ability to produce instrumented programs that can be used in place of their production versions during the system test phase, thus allowing one to conclude that the occurrence of faults in one version corresponds to the occurrence of faults in the other. This has been addressed for sequential program with the requirement that contracts be side-effect free. This requirement is not sufficient to guarantee equivalence in a concurrent environment due to the non-deterministic nature of concurrent programs.

The modifications for the RAC to work in a concurrent environment were made in a way to guarantee that all possible interleavings present in the production version of the system would also be possible to occur in its instrumented version. This was achieved basically by keeping the RAC state in the context of every executing thread. The only situation in which threads need to synchronize was dealt with by keeping state in a concurrent map and using it in a way that maximizes concurrency, thus reducing the probability of any lock contentions and, therefore, increasing the probability of interleavings present in the production version to occur in the instrumented version.

We showed that the excess static and dynamic resources introduced by the RAC can be predicted thus allowing, during system testing, for the appropriate tuning of test bed parameters that influence the load placed on the system under test. We were also able to 
show that both versions of the system present the same behaviour with respect to thread interleaving. These two aspects demonstrate that the RAC can produce instrumented code that behaves the same as its production version.

The effectiveness of concurrent contracts as test oracles was evaluated both with respect to concurrency related faults, namely deadlocks and race conditions, as well as with respect to faults related to their functional aspect. Effectiveness was characterized in terms of two measures: the global observability, which is the probability of oracles detecting faults, and the global diagnosability, which is the average effort to diagnose faults normalized with the size of an execution thread. Both studies were performed in a representative concurrent industrial software system following a precise and objective procedure.

We showed that deadlocks and race conditions can be detected by contracts utilizing the constructs we proposed. We also showed that they are extremely effective since they were able to detect all faults. More importantly, we finally showed they turn the diagnosis process into an extremely easy task by detecting faults very close to their location. These are both significant contributions of this work since the diagnosis of concurrent faults is usually a very difficult task. A subtle but important contribution is showing this is possible using dynamic methods (as opposed to the usual static and generally not scalable methods such as model checking) through a unified notation allowing contracts to describe both the functional and the concurrent aspects of a system.

Regarding functional faults, we showed through experimental data that contracts can be effective test oracles in a concurrent environment. This has so far been conjectured but we were able to clearly demonstrate it by the measured values of observability and diagnosability presented by the instrumented system. The final and likely the most significant contribution of this work, was to create a set of clear and objective guidelines for contracts to satisfy to be effective test oracles balancing the availability of resources to spend in designing contracts with the payoff in increased fault detection and decreased diagnosis effort. Such guidelines are based on structural properties of the contract and the code being specified. We used MacCabe's cyclomatic complexity as the code structural 
property whereas we defined the Contract Complexity Measure to serve as the contract structural property. Both are based on a graph theoretical framework allowing them to be computed automatically. The guidelines were derived assuming the target system is representative of concurrent industrial systems. More research is required to extend these findings to purely sequential software.

This work focused on Java as the system programming language and the Java Modeling Language (JML) as the language to define contracts. We believe, however, that our contributions are applicable to other object-oriented programming and specification languages. Having addressed the problems of specification and instrumentation of concurrent programs, and showing that concurrent and functional faults can be detected by instrumented concurrent software, we showed that Design by Contract can now be applied to concurrent object-oriented systems.

\subsection{Future Work}

As part of the first case study, a limitation was identified regarding the specification of what we named piecewise thread safety of objects. This is an important limitation of the proposed thread-safety constructs since it prevents a significant number of classes to be completely specified. A solution needs to be amenable to inheritance and abstract modeling.

The semantics of specification inheritance on the concurrent aspect of contracts was proposed to address certain requirements we considered important in the specification of thread-safety properties. The question of behavioural subtyping on this aspect is still open. It is our belief that the proposed semantics with maybe minor modifications can be proven to guarantee behavioural subtyping. This is fundamental work that will enable the modular reasoning about object-oriented programs in the concurrent aspect.

In terms of tools, there is considerable room for improving the performance of the RAC to require less memory and $\mathrm{CPU}$. 
In the last case study, we show that the three measures of the diagnostic effort we use, namely the diagnosis scope, the distance and the weighed distance are statistically equivalent, therefore allowing the user to chose the one that best represents the actual effort in diagnosing a fault. This is an open problem with immediate practical application.

The relation between the global observability and global diagnosability was observed to be linear. This is contrary to the available models. The reasons for this discrepancy are unknown. It could be a property particular to concurrent systems. Or the model and the associated simplifying assumptions might be incorrect. The limited amount of data does not allow any such conclusions. Controlled experiments in simpler (smaller) systems might be an interesting first step to gather sufficient data to allow statistical inference on their relation.

A similar experiment regarding observability and diagnosability needs to be performed to determine if the contract guidelines presented can be applied to sequential software.

Extending the use of $\mathrm{DbC}$ to the specification of distributed systems is a natural next step adding a new dimension of complexity to the problem of the specification of concurrency properties. 


\section{REFERENCES}

1. B. Meyer, "Design by Contract," IEEE Computer, vol. 25, no. 10, 1992, pp. 4052.

2. P. Müller, A. Poetzsch-Hefter and G.T. Leavens, "Modular Invariants for Layered Object Structures," Science of Computer Programming, vol. 62, no. 3, 2006, pp. 253-286.

3. B.H. Liskov and J.M. Wing, "A Behavioral Notion of Subtyping," $A C M$ Transactions on Programming Languages and Systems, vol. 16, no. 6, 1994, pp. 18111841.

4. P. America, "Inheritance and Subtyping in a Parallel Object-Oriented Language," Proc. European Conference on Object Oriented Programming, 1987, pp. 234-242.

5. P. America, "Designing an Object-Oriented Programming Language with Behavioural Subtyping," Proc. REX School/Workshop on Foundations of ObjectOriented Languages, 1990, pp. 60-90.

6. G.T. Leavens and W.E. Weihl, "Specification and Verification of Object-Oriented Programs Using Supertype Abstraction," Acta Informatica, vol. 32, no. 8, 1995, pp. 705778.

7. A.D. Raghavan and T.L. Gary, Desugaring JML Method Specifications, TR \#0003e, Iowa State University, 2000.

8. K. Dhara and G.T. Leavens, "Forcing Behavioural Subtyping Through Specification Inheritance," Proc. International Conference on Software Engineering, 1996, pp. 258-267.

9. G.T. Leavens, "JML's Rich, Inherited Specifications for Behavioral Subtypes," Proc. International Conference on Formal Engineering Methods, 2006, pp. 2-34.

10. G.T. Leavens and D.A. Naumann, Behavioral Subtyping, Specification Inheritance, and Modular Reasoning, Technical Report 06-20b, Department of Computer Science, Iowa State University, 2006.

11. K. Arnold, J. Gosling and D. Holmes, The Java Programming Language, Addison-Wesley, 2000.

12. G.T. Leavens, A.L. Baker and C. Ruby, "Preliminary design of JML: A behavioral interface specification language for Java," $A C M$ SIGSOFT Software Engineering Notes, vol. 31, no. 3, 2006, pp. 1-38. 
13. G.T. Leavens, E. Poll, C. Clifton, Y. Cheon, C. Ruby, D. Cok, P. Müller, J. Kiniry, P. Chalin and D.M. Zimmerman, "JML Reference Manual," 2009; http://www.eecs.ucf.edu/ Leavens/JML/jmlrefman/jmlrefman toc.html.

14. Y. Cheon and G.T. Leavens, "A Runtime Assertion Checker for the Java Modeling Language," Proc. Software Engineering Research and Practice, 2002, pp. 322328.

15. G. Weikum and G. Vossen, Transactional information systems : theory, algorithms, and the practice of concurrency control and recovery, Morgan Kaufmann, 2002, p. xxv, 853 p.

16. E. Rodríguez, M. Dwyer, C. Flanagan, J. Hatcliff, G.T. Leavens and Robby, "Extending JML for Modular Specification and Verification of Multi-threaded Programs," Proc. European Conference on Object-Oriented Programming, 2005, pp. 551-576.

17. B. Beizer, Software testing techniques, Van Nostrand Reinhold, 1990, p. xxvi, 550.

18. C. Flanagan and S.N. Freund, "Atomizer: a dynamic atomicity checker for multithreaded programs," Proc. ACM SIGPLAN/SIGACT Symposium on Principles of Programming Languages, 2004, pp. 256-267.

19. K.R.M. Leino, G. Nelson and J.B. Saxe, "ESC/Java User's Manual," 2000; http://secure.ucd.ie/products/opensource/ESCJava2/ESCTools/docs/ESCJAVAUsersManual.html.

20. G.T. Leavens, Y. Cheon, C. Clifton, C. Ruby and D.R. Cok, "How the design of JML accommodates both runtime assertion checking and formal verification," Science of Computer Programming, vol. 55, 2005, pp. 185-208.

21. Y. Cheon, G.T. Leavens, M. Sitaraman and S. Edwards, "Model Variables: Cleanly supporting Abstraction in Design By Contract," Software - Practice and Experience, vol. 35, no. 6, 2005, pp. 583-599.

22. B. Jacobs, R.M. Leino, F. Piessens and W. Schulte, "Safe concurrency for aggregate objects with invariants," Proc. IEEE International Conference on Software Engineering, 2005, pp. 137-147.

23. C.A.R. Hoare, "Monitors: an operating system structuring concept," Commun. $A C M$, vol. $17, \quad$ no. $10,1974, \quad$ pp. 549-557; $\quad$ DOI http://doi.acm.org/10.1145/355620.361161.

24. R. Agrawal, A. Sasturkar, L. Wang and S.D. Stoller, "Optimized Run-Time Race Detection And Atomicity Checking Using Partial Discovered Types," Proc. IEEE International Conference on Automated Software Engineering, 2005, pp. 233-242. 
25. B. Jacobs, R.M. Leino and W. Schulte, "Verification of Multithreaded ObjectOriented Programs with Invariants," Proc. ACM Specification and Verification of Component Based Systems, 2004, pp. 2-9.

26. R.J. Lipton, "Reduction: a method of proving properties of parallel programs," Communications of the $A C M$, vol. 18, no. 12, 1975, pp. 717-721.

27. J. Gosling, The Java language specification, Addison-Wesley, 2005, p. xxxii, 651 p.

28. Y. Cheon and G.T. Leavens, A Runtime Assertion Checker for the Java Modeling Language, TR \#03-09, Iowa State University, Department of Computer Science, 2003.

29. S. Savage, M. Burrows, G. Nelson, P. Sobalvarro and T. Anderson, "Eraser: a dynamic data race detector for multithreaded programs," ACM Transactions on Computer Systems, vol. 15, no. 4, 1997, pp. 391-411.

30. C. von Praun and T.R. Gross, "Object race detection," Proc. ACM SIGPLAN Conference on Object Oriented Programming, Systems, Languages, and Applications, 2001, pp. 70-82.

31. C. Flanagan, K.R.M. Leino, M. Lillibridge, G. Nelson, J.B. Saxe and R. Stata, "Extended static checking for Java," Proc. Proceedings of the ACM SIGPLAN 2002 Conference on Programming language design and implementation, ACM, 2002, pp. 234245.

32. A. Greenhouse, T.J. Halloran and W.L. Scherlis, "Observations on the assured evolution of concurrent Java programs," Science of Computer Programming, vol. 58, no. 3, 2005, pp. 384-411.

33. P. Nienaltowski and B. Meyer, "Contracts for concurrency," Proc. International Symposium on Concurrency, Real-Time and Distribution in Eiffel-like Languages, 2006.

34. V. Arslan, P. Eugster, P. Nienaltowski and S. Vaucouleur, "SCOOP - concurrency made easy," Dependable Systems: Software, Computing, Networks, LNCS 4028, B. Meyer, et al., eds., 2006, pp. 82-102.

35. B. Baudry, Y. Le Traon and J.M. Jezequel, "Robustness and diagnosability of OO systems designed by contracts," Proc. Proceedings Seventh International Software Metrics Symposium. METRICS 2001, 4-6 April 2001, IEEE Comput. Soc, 2000, pp. 272284.

36. Y. Le Traon, B. Baudry and J.M. Jezequel, "Design by contract to improve software vigilance," IEEE Transactions on Software Engineering, vol. 32, no. 8, 2006, pp. 571-586.

37. B. Baudry, H. Vu Le, J.M. Jezequel and Y. Le Traon, "Building trust into OO components using a genetic analogy," Proc. Proceedings of ISSRE 2000 International 
Symposium on Software Reliability Engineering, 8-11 Oct. 2000, IEEE Comput. Soc, 2000, pp. 4-14.

38. L.C. Briand, Y. Labiche and H. Sun, "Investigating the Use of Analysis Contracts to Improve the Testability of Object-Oriented Code," Software - Practice and Experience, vol. 33, no. 7, 2003, pp. 637-672.

39. L.C. Briand, Y. Labiche and H. Sun, "Investigating the use of analysis contracts to support fault isolation in object oriented code," Proc. Proceedings of the 2002 ACM SIGSOFT international symposium on Software testing and analysis, ACM, 2002, pp. 7080.

40. D. Coleman, Object-oriented development : the fusion method, Prentice Hall, 1994, p. xx, 313 p.

41. K. Chan, J. Seligson, D. Durham, S. Gai, K. McCloghrie, S. Herzog, F. Reichmeyer, R. Yavatkar and A. Smith, RFC3084: COPS Usage for Policy Provisioning (COPS-PR), IETF, 2001.

42. D. Durham, J. Boyle, R. Cohen, S. Herzog, R. Rajan and A. Sastry, RFC2748: The COPS (Common Open Policy Service) Protocol, IETF, 2000.

43. OMG, The Common Object Request Broker: Architecture and Specification. Version 3.0, formal/02-06-01, Object Management Group, 2002.

44. T. Lindholm and F. Yellin, The Java virtual machine specification, AddisonWesley, 1999, p. xv, 473 p.

45. T.J. McCabe, "Complexity Measure," IEEE Transactions on Software Engineering, vol. SE-2, no. 4, 1976, pp. 308-320.

46. E.J. Weyuker, "Empirical study of the complexity of data flow testing," 1988, pp. 188-195.

47. L.C. Briand, S. Morasca and V.R. Basili, "Property-based software engineering measurement," IEEE Transactions on Software Engineering, vol. 22, no. 1, 1996, pp. 6886. 


\section{APPENDIX}

\subsection{Obtaining the Software}

This research was conducted on the JML2 line of JML tools. The modifications made to the JML toolset (the JML checker, JML compiler and JML RAC runtime) were not integrated in the main release branch at the time of publicizing. To obtain a copy of the software used to perform these experiments, one should follow the instructions in the JMLSpecs site (http://www.eecs.ucf.edu/ leavens/JML/development.shtml) to obtain access to the CVS repository.

To check out the appropriate version of JML simply issue the appropriate CVS command to check out sources from the branch named "waraujo_concurrency". The version of the MultiJava project must match the version of the JML tools since some minor modifications were necessary in the code generation routines. The matching version of MultiJava is also in a branch named "waraujo_concurrency".

Detailed instructions in using CVS to develop JML2 and to build it from the sources can be found at http://www.eecs.ucf.edu/ leavens/JML/developer-tips.shtml.

\subsection{Using the Software}

The JML compiler ( $j m l c$ ) was extended with the --multithread or -m command line interface (CLI) options. If this option is not specified, jmlc will generate code using the sequential semantics. The JML checker (and, consequently, jmlc), however, will still recognize and type-check the added clauses. It will simply ignore the concurrency related clauses during the code-generation phase. The compiler was also extended with the -noThrow or $-z$ CLI options, which cause log messages to be printed to stderr instead of throwing JMLAssertionError exceptions in case of assertion violations.

Due to the nature of the build environment in which the experiments were carried out (i.e. Juniper Networks's development environment for the SRC product), it was necessary to have jmlc output Java code to be later compiled by the Java compiler. Therefore, all 
experiments were performed using the --print or -P CLI options, and thus the bytecode generation of concurrency RAC code has not been extensively tested. The tests and experiments were all performed using the efficient RAC semantics, which is the default option for later versions of jmlc but is not on the "waraujo_concurrency" branch. Therefore, options --efficientRAC or $-T$ need to be specified explicitly at compilation time.

The jmlrac script (used to execute a Java program with RAC) has been modified to include the JMLThreadSafety Instrumenter class (see section 4.4) as a Java Agent (see http://java.sun.com/javase/6/docs/api/java/lang/instrument/package-summary.html) to insert bytecode for checking of thread-safety properties in pre-compiled classes. This class accepts two optional bootstrap options: includePrefix and excludePrefix, which specify the class name prefixes in path format (e.g. java/net) to be included in and excluded from, respectively, the instrumentation process. These options must be specified according to the following expression in EBNF (Figure A.1) where prefix denotes a string that is acceptable as a partial pathname.

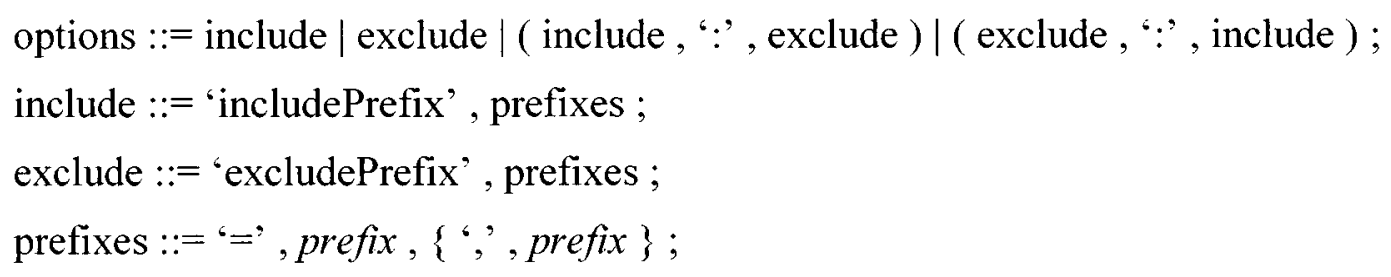

Figure A.1: CLI options in EBNF format for the jmlrac utility to perform threadsafety instrumentation of pre-compiled classes.

The thread-safety instrumenter implicitly excludes the following prefixes: java/lang/, java/security/, com/sun/, sun/, sunw/, org/jmlspecs/jmlrac/runtime/, java/util/concurrent/locks/, java/net/URLClassLoader. It also implicitly includes the prefix java/util/. Exclusion takes precedence over inclusion. For instance the contents of the java.util package and sub-packages are included except for the java.util.concurrent.locks package. 


\subsection{Contract and Method Complexity Distributions for the Target System of the Presented Case Studies}

Figure A.2 shows the distribution of the detected faults per the VG of the methods in which they were found. Figure A.3 shows the distribution of methods VG. These are the raw data used to construct the graph in Figure 18.

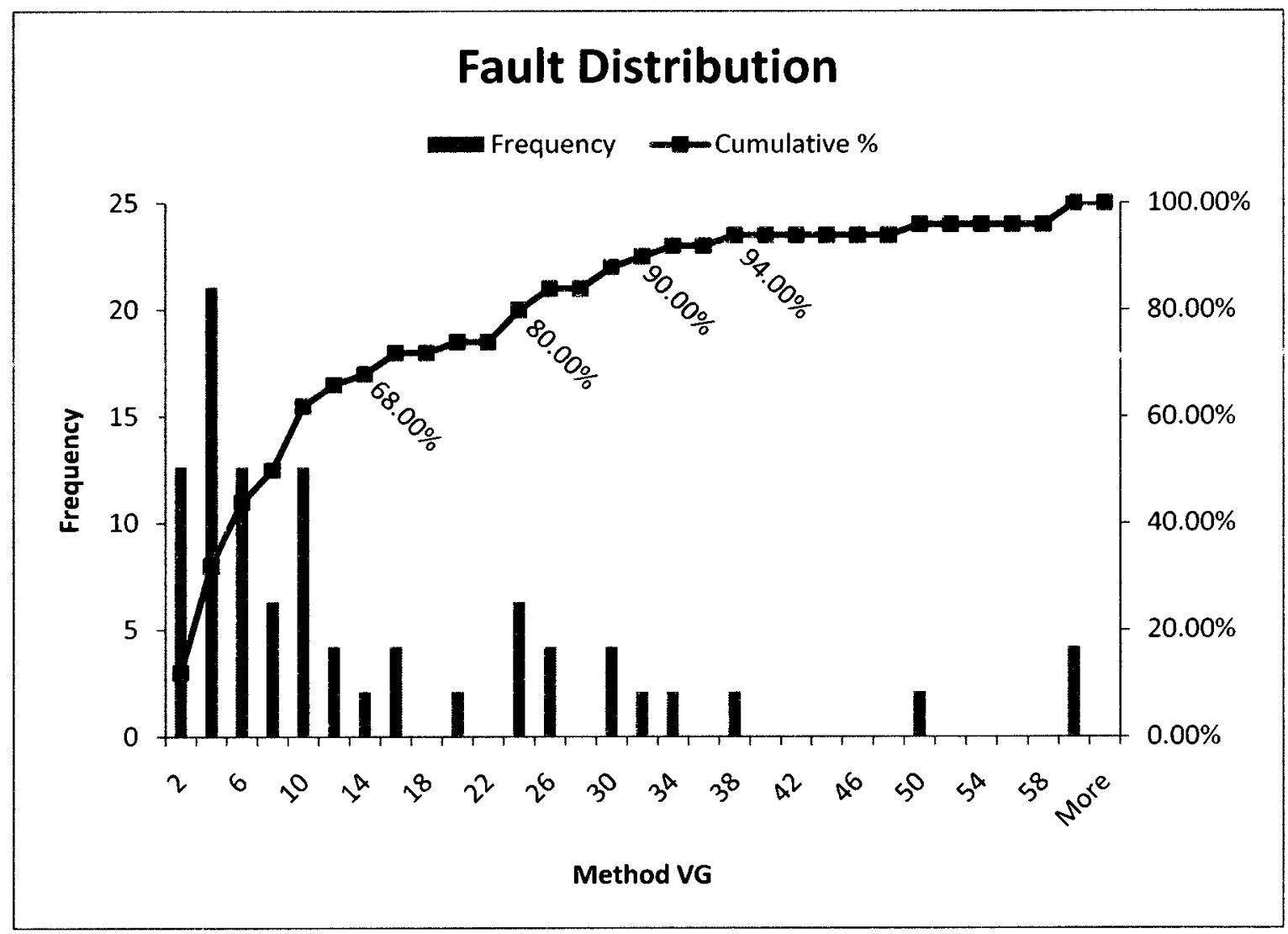

Figure A.2: Histogram of faults detected per method cyclomatic complexity. 


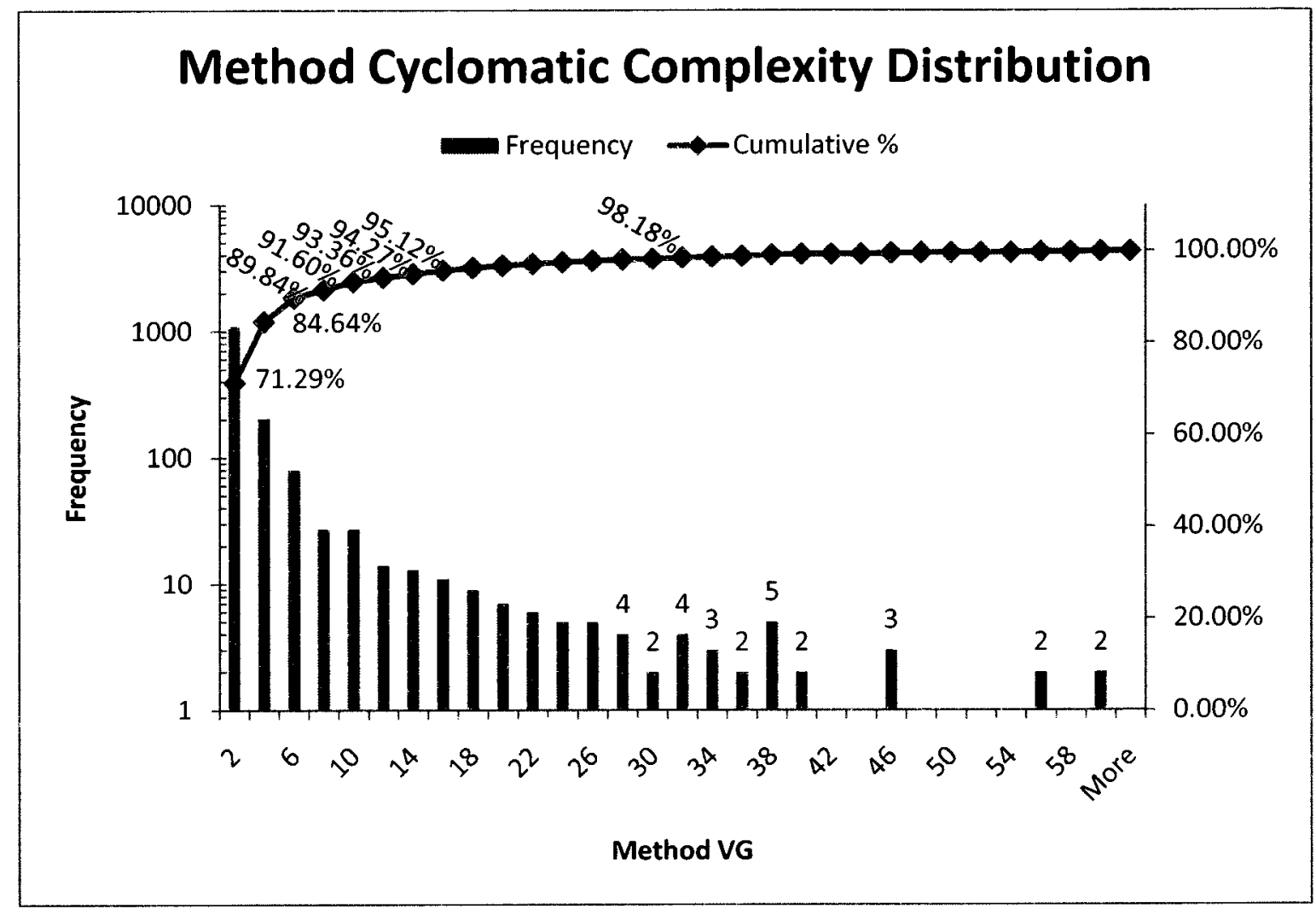

Figure A.3: Histogram of methods per cyclomatic complexity in the system under test. The frequency axis is in logarithmic scale. 